Doctoral School of Multidisciplinary Medical Sciences

Department of Biochemistry

Faculty of Medicine

UNIVERSITY OF SZEGED

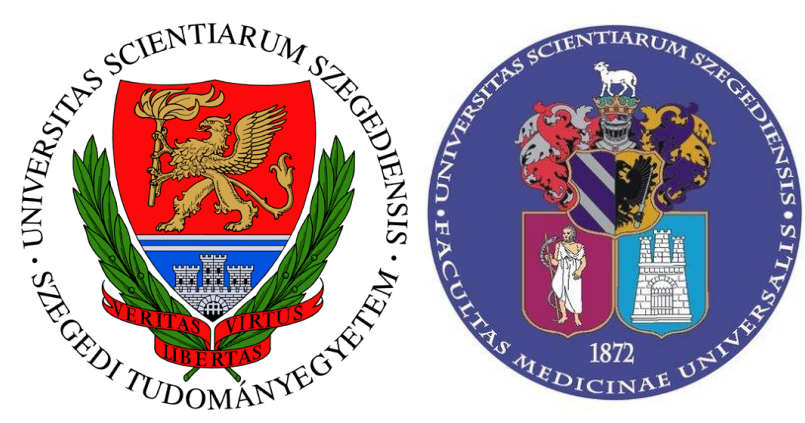

Ph.D. Thesis

Multiple roles of syndecan-4 during myoblast migration

\author{
Dániel Becsky
}

Supervisor: Anikó Keller-Pintér MD Ph.D. 


\section{Full length publications related to the subject of the Ph.D. thesis}

I. Daniel Becsky*, Szuzina Gyulai-Nagy*, Arpad Balind, Peter Horvath, Laszlo Dux, Aniko Keller-Pinter (2020) Myoblast Migration and Directional Persistence Affected by Syndecan-4-Mediated Tiam-1 Expression and Distribution. Int J Mol Sci. 21: 823. [IF: 4.556] Q1

II. Daniel Becsky*, Kitti Szabo*, Szuzina Gyulai-Nagy, Tamas Gajdos, Zsuzsa Bartos, Arpad Balind, Laszlo Dux, Peter Horvath, Miklos Erdelyi, Laszlo Homolya, and Aniko Keller-Pinter (2020) Syndecan-4 Modulates Cell Polarity and Migration by Influencing Centrosome Positioning and Intracellular Calcium Distribution. Front Cell Dev Biol. 8: 575227. [IF: 5.201] Q1

*: contributed equally

Cumulative impact factors of papers directly related to the subject of the thesis: 9.757 


\section{TABLE OF CONTENTS}

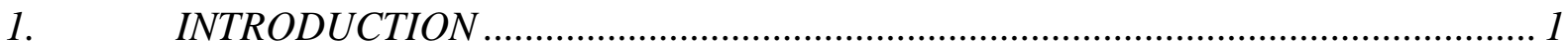

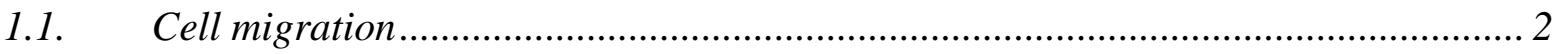

1.2. Cytoskeletal dynamics and migration ................................................................ 3

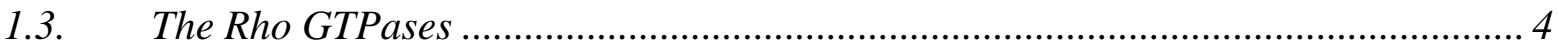

2. GAPS IN CURRENT KNOWLEDGE: AIMS OF THE THESIS ........................... 11

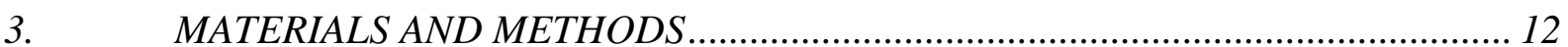

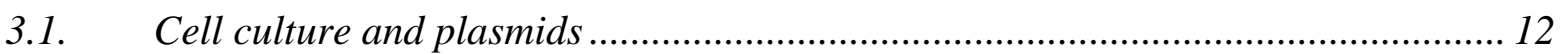

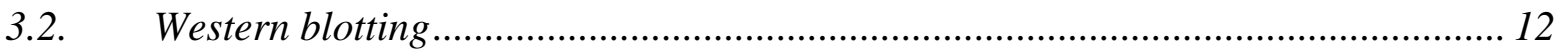

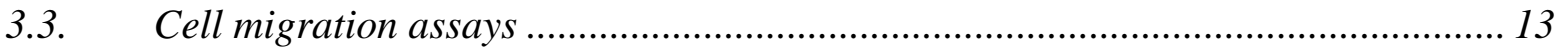

3.4. Time-lapse imaging of the migration of live cells ................................................ 14

3.5. $\quad$ Single-cell tracking of cultured myoblasts ............................................................. 15

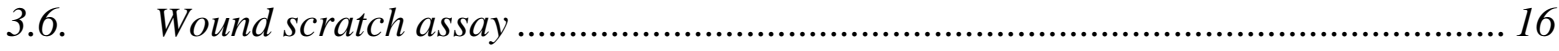

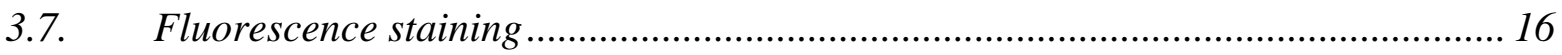

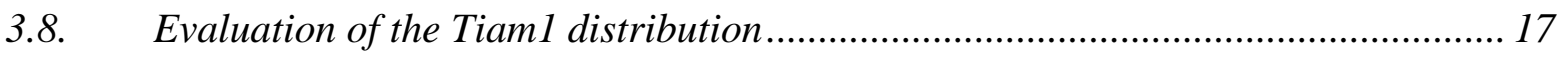

3.9. Determining and quantifying the position of centrosomes .................................... 17

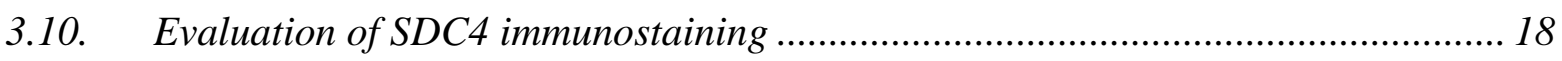

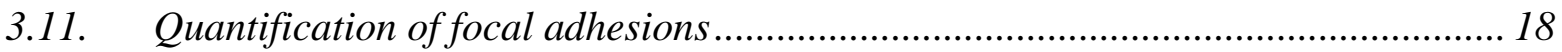

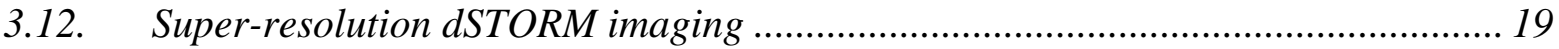

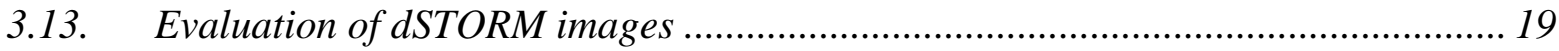

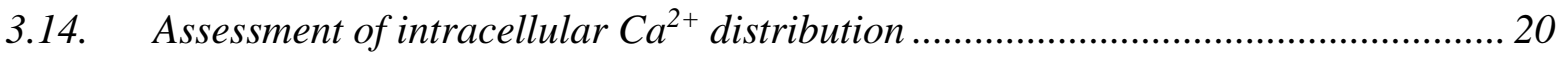

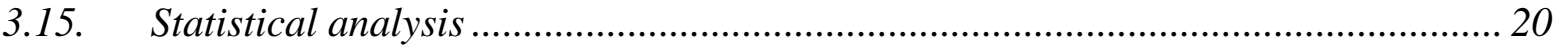

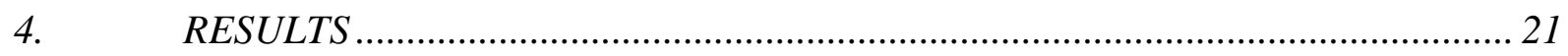

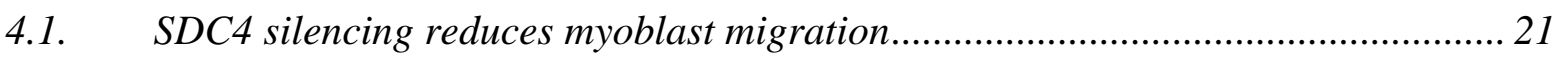

4.2. SDC4 affects the nanoscale architecture of the actin cytoskeleton, as determined by super-resolution dSTORM ................................................................................... 23

4.3. Effect of SDC4 knockdown on focal adhesions .................................................. 28

4.4. SDC4 affects centrosome positioning and cell polarity ........................................ 30

4.5. Polarized distribution of SDC4 during migration .................................................... 33

4.6. SDC4 knockdown abrogates the intracellular $\mathrm{Ca}^{2+}$ gradient in migrating cells ... 35

4.7. Inhibition of Racl does not restore the defective migratory phenotype of SDC4

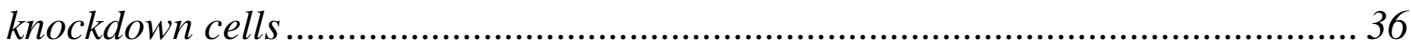

4.8. SDC4 affects Tiaml expression and localization ................................................ 37

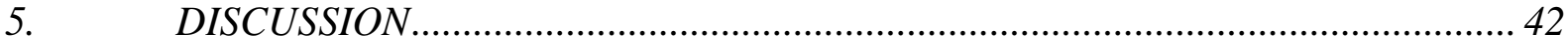




\section{LIST OF ABBREVIATIONS}

ADAM: A disintegrin and metalloprotease

C1 (region): Conservative region 1 of Syndecan- 4 cytoplasmic domain

C2 (region): Conservative region 2 of Syndecan- 4 cytoplasmic domain $\mathrm{Ca}^{2+}:$ Calcium

CASK: Calcium/calmodulin-dependent serine protein kinase

Cdc42: Cell division control protein 42 homolog

dSTORM: Direct stochastic optical reconstruction microscopy

EDTA: Ethylenediaminetetraacetic acid

Ferm: Four-point-one protein, ezrin, radixin, moezin

FGF: Fibroblast growth factor

GAG: Glycosaminoglycan

GEF: Guanine nucleotide exchange factor

GM130: Golgi matrix protein of $130 \mathrm{kDa}$

HS-GAG: Heparan sulphate-glycosaminoglycan

MMP: Matrix metalloproteinase

Par: Partitioning-defective (polarity complex)

PDGF: Platelet-derived growth factor

PDZ: Postsynaptic density protein

PIP2: Phosphatidylinositol-4,5-bisphosphate

PKC: Protein kinase C

Rac1: Ras-related C3 botulinum toxin substrate 1

RhoA: Ras homolog family member A

SCs: Stem cells

SDC: Syndecan

SDC1: Syndecan-1

SDC2: Syndecan-2

SDC3: Syndecan-3

SDC4: Syndecan-4

Ser: Serine

shRNA: Short hairpin RNA

Tiam1: T-lymphoma invasion and metastasis-1

TRPC: Transient receptor potential cation channel

V (region): Variable region of Syndecan-4 cytoplasmic domain

VEGF: Vascular endothelial growth factor

WASP: Wiskott-Aldrich Syndrome protein 


\section{SUMMARY}

Background and purpose: Cell migration is one of the cornerstones of regeneration processes, as it is necessary for wound healing, and also required for embryonic development, immune system activation, or tumor metastasis formation. Skeletal muscle has a special, advanced dynamism that allows it to adapt to various impacts and recover successfully after an injury, exercise, or muscle disease. Satellite stem cells are activated by local damage during muscle regeneration, and after asymmetric division, myoblasts (i.e., activated satellite cells) migrate to the site of injury, differentiate, and fuse to form muscle fibers. Migration of the cells requires cellular polarization, the creation of leading and trailing edges, as well as the proper orientation and positioning of organelles inside the cell. Efficient migration also requires the presence of an asymmetrical front-to-rear calcium $\left(\mathrm{Ca}^{2+}\right)$ gradient to regulate focal adhesion assembly and actomyosin contractility. The transmembrane proteoglycan syndecan-4 (SDC4), which is one of the cell surface markers of resting and activated satellite stem cells, is involved in the formation of focal adhesions. Furthermore, SDC4 plays a variety of roles in signal transduction processes, including controlling the function of the small GTPase Rac1 by binding to and inhibiting the activity of T-lymphoma invasion and metastasis-1 (Tiam1), a guanine nucleotide exchange factor for Rac1 (Ras-related C3 botulinum toxin substrate 1) GTPase. Cell migration also requires Rac1-mediated actin remodeling. SDC4 knockout mice are unable to regenerate damaged muscle; however, its underlying precise mechanism is unclear; therefore, our aim was to analyze the role of SDC4 in myoblast migration.

Experimental approaches: To achieve SDC4 knockdown, C2C12 murine myoblast cells were transfected stably with plasmids expressing short hairpin RNAs (shRNAs) specific for mouse SDC4 (shSDC4\#1 and shSDC4\#2) or a scrambled target sequence. To study cell migration, time-lapse images were captured at $37{ }^{\circ} \mathrm{C}$ and $5 \% \mathrm{CO} 2$ using a high-content imaging system for single-cell tracking or wound scratch assay was performed. To evaluate the movement of the single cells, the cell nuclei were tracked with ImageJ and CellTracker software. Super-resolution direct stochastic optical reconstruction microscopy (dSTORM) measurements were performed for the nanoscale analysis of the lamellipodial actin network of the migrating cells. To study the intracellular $\mathrm{Ca}^{2+}$ level, Fluo- 4 and Fura Red indicators were applied. Immunofluorescence cytochemistry was performed to analyze the distribution of SDC4, Tiam1, centrosomes, FAK (focal adhesion kinase) or GM130 (anti- Golgi matrix protein of $130 \mathrm{kDa}$ ) followed by wide-field fluorescence or confocal microscopy. Image 
analysis was performed with ImageJ. Rac1 was inhibited by NSC23766 treatment during the measurements $(50 \mu \mathrm{M})$.

Key results: Silencing of SDC4 disrupts the correct polarization of migrating mammalian myoblasts. SDC4 knockdown completely abolished the intracellular $\mathrm{Ca}^{2+}$ gradient, abrogated centrosome reorientation, and thus decreased cell motility, demonstrating the role of SDC4 in cell polarity. Additionally, SDC4 exhibited a polarized distribution during migration. SDC4 knockdown cells exhibited decreases in the total movement distance during migration, maximum and vectorial distances from the starting point, as well as average and maximum cell speeds. Analysis of the dSTORM images of SDC4 knockdown cells revealed nanoscale changes in the actin cytoskeletal architecture, such as decreases in the numbers of branches and individual branch lengths in the lamellipodia of the migrating cells. The Rac1 inhibitor NSC23766 did not restore the migration capacity of SDC4 silenced cells; in fact, it reduced it further. SDC4 knockdown decreased the directional persistence of migration, abrogated the polarized, asymmetric distribution of Tiam1, and reduced the total Tiam1 level of the cells.

Conclusion: According to our results, SDC4 affects the migration of $\mathrm{C} 2 \mathrm{C} 12$ myoblasts and modulates cell polarity by influencing centrosome positioning, intracellular $\mathrm{Ca}^{2+}$ and Tiam1 distribution. These findings may promote greater understanding the essential role of SDC4 in the embryonic development and postnatal regeneration of skeletal muscle. Given the ubiquitous expression and crucial role of SDC4 in cell migration, we conclude that our findings can facilitate understanding the general role of SDC4 during cell migration. 


\section{INTRODUCTION}

Skeletal muscle can adapt to different impacts with a unique, advanced dynamism, and it is able to regenerate successfully following an injury. It can also change in size due to aging, exercise, or different diseases (e.g., cancer cachexia, immobilization, muscular dystrophy). Stem cells of skeletal muscle, so-called satellite cells (SCs) - which are located between the muscle fiber and basal lamina - are responsible for the plasticity, maintenance and regeneration of skeletal muscle $[1,2]$. They are both mitotically and physiologically quiescent until they are activated by local injury. Thereafter myoblasts are formed, which proliferate and also migrate to the area of injury. Then they differentiate and form a multi-nucleated syncytium with common cytoplasm called myotube (fig. 1.).

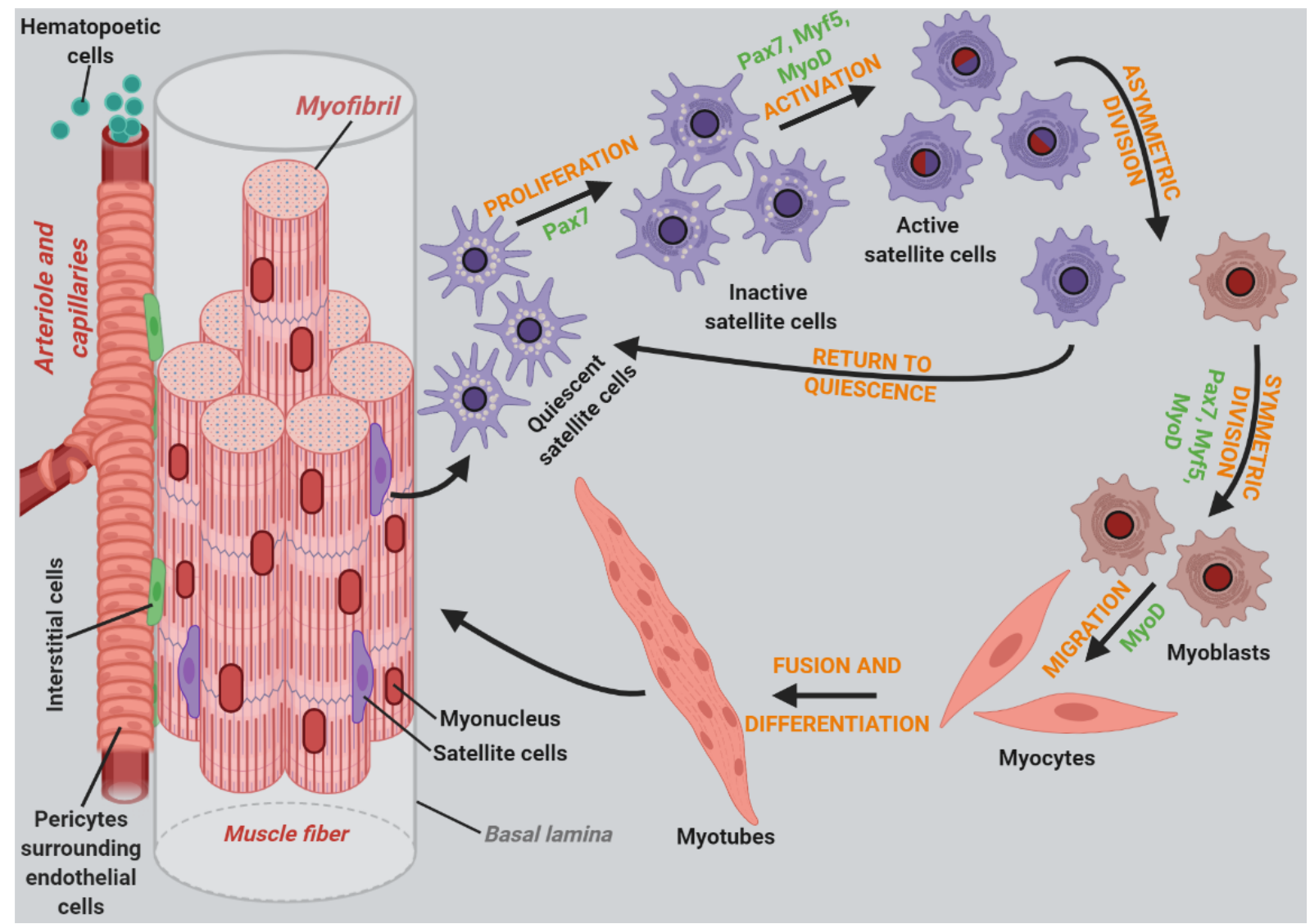

Figure 1. Structure and regeneration of skeletal muscle. The myofibre is the structural unit of the skeletal muscle, which is a synctitial reticulation of myocytes enwraped by the basal lamina or endomysium. Fused cells with myonuclei, mitochondria and myofibrils with contractile myofilaments are included in the myofibers. The mononuclear satellite cells (SCs) can be found between the plasma membrane of myofibrils and basal lamina. SCs activation in vivo is followed by an asymmetric division, with Pax7, MyoD, and Myf5 being expressed in differentiating cells and Pax7 in cells returning to quiescence in order to maintain a pool of progenitors [3,4]. The image is created by BioRender app. 


\subsection{Cell migration}

Cell migration is essential for establishing and maintaining the proper organization of multicellular organisms [5]. This migration is a fundamental process that is essential in a variety of physiological and pathological tasks such as regeneration, wound healing, angiogenesis, embryonic development, and immune cell responses, as well as tumor progression and metastasis formation.

For the process of cell migration to occur, a rearrangement of the cytoskeleton and cell matrix adhesions is required. The cytoskeletal system consists of microtubules, intermediate filaments, and microfilaments. Microtubules are structures composed of $\alpha$ and $\beta$ tubulin subunits, they determine the shape of cells and are actively involved in directing the movement of cells. Intermediate filaments, which are smaller in diameter, provide mechanical stability to cells. The microfilaments or actin filaments are the smallest in diameter. The actin cytoskeleton is a dynamic structure, actively involved in cell movement. During migration, the front of the cell defines the direction of movement as a tail region forms, causing the morphology of the cells to change, forcing them to elongate as a result of actin-cytoskeleton and cell-matrix rearrangement. The shaped tail region is known as the trailing edge, while the front region as the leading edge (fig. 2.).

The cell-matrix contact points, focal adhesions, are dynamic structures made up of over 150 proteins. Cell migration requires the continuous assembly and disassembly of focal adhesions. New focal adhesions form at the front and disrupt at the tail, causing the cell to move [6].

Cells become polarized in the early stages of cell migration, and protrusions (i.e., sheetlike lamellipodia and finger-like filopodia) develop on the cell's leading edge. Actin stress fibers form in migrating cells and play an important role in cell contractility, mechanical strength, and are also involved in cell adhesion and migration. The main components of these stress fibers are the actin microfilaments, myosin II (mechanochemical enzyme) and $\alpha$-actinin (actin-binding protein). There are three main types of stress fibers in the migrating cell: ventral stress fibers, transverse arcs, and dorsal stress fibers. Ventral stress fibers are associated with focal adhesion at both ends and are located on the tail region of the cell [6]. Transverse arcs do not connect directly to focal adhesions and usually stream back from the anterior edge of the cell toward the center. The dorsal stress fibers are located on the front edge of the cell. They attach to focal adhesions on the ventral surface of the leading edge, and extend dorsally, towards the cell center to attach to transverse arcs. During cell migration, the 
actin fibers are recycled by a retrograde actin flow process, creating a dynamically active cyclic system $[7,8]$.

The existence of asymmetry within a cell is referred to as cell polarity. To conduct specialized cell functions, cells must acquire specific types of polarity. This polarization of the cells, morphologically the formation of the front-rear edge, and the proper orientation of the cellular components, is one of the most remarkable conditions for cell movement during tissue development [6]. In the lamellipodium that develops in the direction of migration, actin accumulates, thus creating a front-rear asymmetry within the cell [9]. During polarization, the relative position of the nucleus and cell organelles (e.g., the Golgi network and the microtubule organizing center) is determining [10,11].

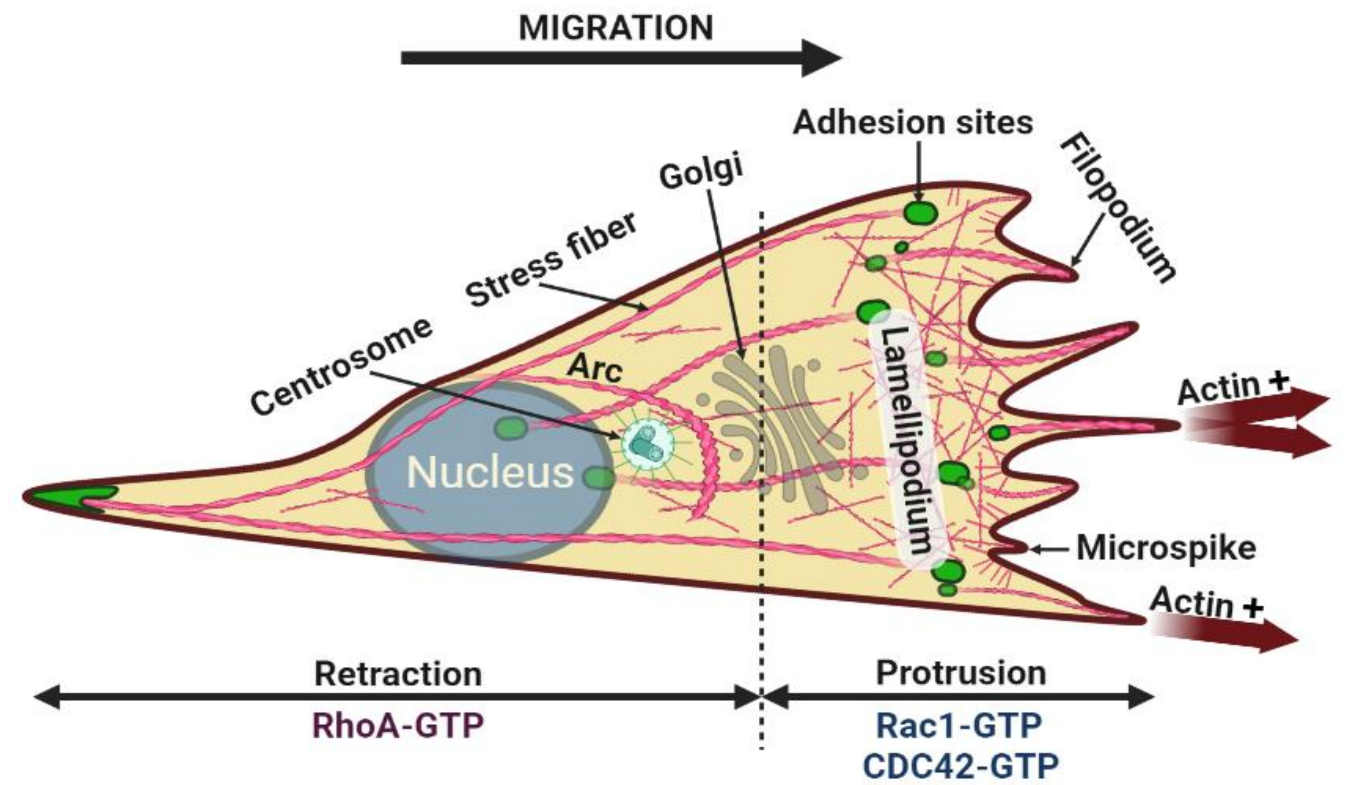

Figure 2. Schematic representation of a polarized migrating cell. Contractile bundles of actin (stress fibers) in the cell body and at the cell edge are represented and a loose actin network throughout the cell. Arc-shaped bundles are sometimes observed that move inwards under the dorsal cell surface (Arc). At the cell front, in lamellipodia and filopodia, actin filaments are all polarized with their fast-polymerizing ends forwards (for pushing); in the body of the cytoskeleton, actin filaments form bipolar assemblies with myosin to form contractile arrays (for retracting). Image created by BioRender app.

\subsection{Cytoskeletal dynamics and migration}

Actin polymerization, retrograde actin network flow, and myosin II-based contractility are all essential in cell migration [12,13]. Cells move by repeating cycles of cell front protrusion and attachment, followed by rear decoupling and retraction. Coordinated polymerization of multiple actin filaments generates protrusive forces that drive plasma membrane protrusion to the cell's leading edge [14]. Contractile force is generated by myosin motors. Not only the active fibers, but also the cells' posterior ends, are pulled back by this 
force. Muscle contraction is identical to this process [15,16]. Actin stress fibers boost cell-cell adhesion as well as cell-extracellular matrix adhesion. They are crucial for preserving and changing the cell's shape, as well as determining the mechanical properties of the cell surface. The "dendritic nucleation" process is a mechanism of actin turnover in lamellipodia that involves the Arp2/3 complex continuously nucleating new actin filaments alongside preexisting "primordial" filaments $[17,18]$. After that, the newly formed filaments elongate and push against plasma membrane. Their barbed end is "capped" after a brief period of elongation, thus elongation is terminated. Disassembly of the network occurs through a combination of debranching and severing of actin filaments, followed by depolymerization of filament fragments. Therefore, individual filaments in the network are created at a branchpoint. They grow at the barbed end, become capped, and later depolymerized [14]. Overall, it can be said that the array of branched filaments in lamellipodia undergoes treadmilling by assembling at the front and disassembling throughout its body. Cadherin complexes regulate actin dynamics mainly via $\alpha$-catenin, which inhibits Arp2/3-mediated branching polymerization [19] and recruits the actin nucleator formin to adherens junctions. In addition to their role of providing junctional stability, $\beta$-catenin and p120-catenin can act as transcriptional regulators [20]. The key organizers of the actin cytoskeleton dynamics are the members of Rho family of small GTPases.

\subsection{The Rho GTPases}

The Rho family of small GTPases including Rac1 (Ras-related C3 botulinum toxin substrate 1), Cdc42 (Cell division control protein 42 homolog), and RhoA (Ras homolog family member A) are evolutionarily conserved regulators of numerous types of cell polarity and the actin cytoskeleton. Rho GTPases function as molecular switches alternating between inactive GDP-bound and active GTP-bound form, they are able to bind and activate downstream effector proteins, thereby exercise an influence on different signaling pathways [21]. The two-state cycle is regulated by three sets of proteins: the guanine nucleotide exchange factors (GEFs), the GTPase-activating proteins (GAPs), and the guanine dissociation inhibitors (GDIs). The GEFs catalyze the exchange of GDP for GTP. GAPs are able to increase intrinsic GTP hydrolysis and are responsible for switching between the active and inactive forms of Rho GTPases. Alternating between GDP- and GTP-bound states may involve cytosol-membrane translocation, as GDIs prevent Rho GTPases from membranetargeting and activation [22]. 
The Rho GTPases have primary importance in the establishment and sustenance of the front-rear polarity in migrating cells [23]. Furthermore, they play a role in cell division, cell morphogenesis, differentiation and understandably migration formation and maintenance [24]. Activated Rac1 is enriched along the leading edge, its activation increases actin polymerization. This process leads to the formation of lamellipodial membrane protrusions [25], while Rac1 activity decreases in the tail region of the cell [6,26]. RhoA activity is highest in the tail region and leads to the appearance of contractile actin bundles (stress fibers). RhoA activity also influences the development of mature focal adhesions [27]. Activation of Cdc42 leads to the appearance of the filopodia [25,27]. Both Rac1 and Cdc42 are able to activate the Arp2/3 complex, leading to actin polymerization and the formation of a branched lamellipodial actin network. As cell movement progresses, focal adhesions reassemble behind the leading edge presumably reflecting the upregulation of Rho, thus providing attachment sites to anchor actin stress fibers and to support the contractile forces necessary for continued cell movement. $\mathrm{Cdc} 42$ and Rac1 regulate the polymerization of cortical actin via their interaction with members of the Wiskott-Aldrich Syndrome protein (WASp)/Scar1 superfamily [28]. Interaction of Cdc42/Rac1 with WASp/Scar proteins unmasks a C-terminal region which mediates binding of WASp/Scar to the Arp $2 / 3$ complex [29]. Arp2/3 complex binds to the sides of preexisting actin filaments and stimulates new filament formation to create branched actin networks [18]. Actin nucleation which is induced by Arp $2 / 3$ is enhanced by binding of WASp-family (acidic) carboxyl-terminal domains to the Arp2/3 complex [30] and such way acts as a molecular link for Cdc42 and Rac1 induction of cortical actin polymerization [29,31]. Rho GTPases play a role not only in the regulation of actin polymerization, but they also involved in actin depolymerization. Rac1 and RhoA also regulate cofilin activity thereby affecting actin depolymerization [32].

Tiam1 (T-lymphoma invasion and metastasis-1) has been identified as a GEF, acting as a specific activator of Rac1 [33], and it plays a key role in pivotal biological processes including cell migration [21] and cell polarization [33]. Tiam1 regulates actin polymerization and actin cytoskeleton rearrangement through its interaction with the Arp2/3 complex [34]. SDC4 affects Rac1 activation through PKC $\alpha$ and accumulates active Rac1 at the leading edge of the migrating cell, thus ensuring the formation of membrane extensions $[35,36]$. The Par (partitioning defective) polarity complex, comprising Par3, Par6 and atypical PKC (protein kinase C), plays a key role in the development and maintenance of cell polarity [21]. Furthermore, Tiam1, along with the Par polarity complex, stimulates persistent migration by stabilizing the anterior-posterior polarization of migrating cells [37]. Par3 interacts with 
Tiam1, leading to localized Rac1 activation, creating a gradient of Rac1 and RhoA GTPases in migrating cells: the former is concentrated at the leading edge, and the latter is in the rear of the cell [38]. As Tiam1-mediated Rac1 signaling is required for establishing and maintaining cell polarity [39], impaired Tiam1 signaling inhibits the formation of front-rear polarization in migrating cells thereby inhibiting persistent migration.

\subsection{The role of $\mathrm{Ca}^{2+}$ in cell migration}

Intracellular $\mathrm{Ca}^{2+}$ plays a major role in cell migration [40-42]. Both $\mathrm{Ca}^{2+}$ influx from the extracellular space through different $\mathrm{Ca}^{2+}$ channels of plasma membrane [43] and $\mathrm{Ca}^{2+}$ release from intracellular stores (primarily the endoplasmic reticulum) contribute to cytosolic $\mathrm{Ca}^{2+}$ concentration [44]. Migrating cells create a front-to-back $\mathrm{Ca}^{2+}$ gradient that is an essential coordinator for the polarized distribution of molecules [40]. This increasing front-rear $\mathrm{Ca}^{2+}$ gradient is involved in the disassembly of focal adhesions and, consequently, the rear-end retraction and the movement of the cell. This essential front-rear polarity is maintained by restricting the spontaneous formation of lamellipodia at the trailing edges of migrating cells [45,46]. In addition to contractility, changes in intracellular $\mathrm{Ca}^{2+}$ affect the activity of calmodulin-dependent enzymes and actin-crosslinking proteins, thus playing a key role in the assembly of adhesions and multilevel junctions [47-49]. High levels of RhoA activity and subsequent actomyosin contractility define the rear of a migrating cell as well as an increased $\mathrm{Ca}^{2+}$ concentration and the activation of $\mathrm{Ca}^{2+}$-dependent proteases required to cleave focal adhesion proteins. It was suggested by Tsai et al. (2015) that the presence of crosstalk between $\mathrm{Ca}^{2+}$ signaling and Rho GTPases would coordinate the oscillations of these factors in the leading edges of migrating cells [45].

\subsection{General overview of syndecans}

Syndecans (SDCs) are type I transmembrane proteoglycans [50], four types are known in vertebrates. The expression of SDCs is cell-, tissue-, and development-specific. Syndecan-1 (SDC1), also known as CD-138, occurs in endothelial, epithelial, smooth muscle and plasma cells. Syndecan-2 (SDC2), also known as fibroglycan, is expressed mainly in fibroblasts, mesenchymes, while syndecan-3 (SDC3) (N-syndecan) is expressed in neurons. Syndecan-4 (SDC4, ryudocan), unlike other members of the family, is universally expressed and is present in virtually all cell types [51] in a development state specific manner; moreover, is a cell surface marker of resting and activated satellite cells [52]. The structure of SDCs consists of 
three parts. N-terminal, which is a variable extracellular domain (ectodomain), the highly conserved transmembrane domain, and the C-terminal intracellular domain. Glycosaminoglycan (GAG) side chains are attached to the backbone protein extracellularly [50,53]. Due to their transmembrane structure, the most important task of the members of the SDC family is to maintain communication between the extracellular matrix and the cell.

Near the N-terminal end of the ectodomain, heparan sulfate chains (GAG chains) are linked via a tetrasaccharide linker to one of the serine (Ser) residues in the ectodomain by an O-glycosidic bond [53]. Chondroitin sulfate side chains bind closer to the transmembrane region for SDC1 and SDC3 [54]. The extracellular domain has a plenty of interacting partners, such as matrix proteins, e.g. fibronectin, matrix metalloproteinases (MMPs), growth factors and cytokines; therefore, SDCs can function as both receptors and co-receptors. The ectodomain can also promote the adhesion and penetration of bacteria and viruses [55-58]. The ectodomain can be cleaved by proteolytic enzymes (secretases), e.g. members of ADAM (disintegrin and metalloproteinase) family and matrix metalloproteinases (MMPs), during the process of shedding. Shedding also plays a role in pathophysiological processes, including inflammation and tissue regeneration [56,59-61]. The transmembrane domain, on the other hand, is the most conserved part of the molecule and also shows a great deal of similarity within the family. It contains a GXXXG motif that strongly influences the formation of SDS (sodium dodecyl sulfate) resistant dimers. [62,63].

The cytoplasmic domain is short and comprised a variable $(\mathrm{V})$ region that is unique to each SDC member but identical within a species. Respectively, it consists of the conservative region preceding $(\mathrm{C} 1)$ and following $(\mathrm{C} 2)$ the $\mathrm{V}$ region [64,65]. The $\mathrm{C} 1$ region can bind to members of the FERM (four-point-one protein, ezrin, radixin, moezin) family, which are membrane- and actin-associated proteins, and bind Src kinase and cortactin [66]. The variable region of SDC4 also binds to PIP2 (phosphatidylinositol-4,5-bisphosphate), which activates PKC-alpha and is associated with alpha-actinin, through which actin binds to the cytoskeleton $[67,68]$. In the C2 region of the cytoplasmic domain of SDCs, the EFYA motif bind proteins containing the PDZ (postsynaptic density protein) domain [64]. The PDZ domain is a submembrane complex of 80-90 amino acids that plays a role in signaling and is one of the most common structural domains. Examples of proteins containing such PDZ domains are synthenine, synectin, synbindine, and CASK (calcium / calmodulin-dependent protein kinase) [69]. 


\subsection{Characterization of SDC4}

SDC4 along with other members of the family, is involved in signal transduction processes across the cell membrane. SDC4 is structurally very similar to other members of the family, however, a significant difference is that, unlike other SDCs, it is universally expressed and present in virtually all cell types. It plays a major role in cell proliferation, migration, cell adhesion, and is also involved in endocytosis and mechanotransduction [51,70-75]. Each of the molecules attached to the GAG chains of SDC4 has a heparin-binding domain, just like FGF2 (fibroblast growth factor-2), VEGF (vascular endothelial growth factor), or PDGF (plateletderived growth factor) [76-80]. In addition, extracellular matrix components, proteases, protease inhibitors, and molecules with tyrosine kinase activity may be interacting partners. By directly binding fibronectin, it is involved in cell adhesion [81], thereby also influencing cell migration.

Due to its indirect relationship with integrins, SDC4 plays an important role in focal adhesion. In the formation of focal adhesions, the heparin binding domain of fibronectin binds to the heparan sulfate side chains of SDC4 $[82,83]$. Fibronectin forms a bridge between SDC4 and integrin (fig. 3.).

SDC4 is involved in several signaling pathways and functions as a structural protein. The $\mathrm{V}$ region of SDC4 binds PKC $\alpha$ and regulates its activity [84,85]. SDC4 dimer forms a tetramer with 2 PIP2 (phosphatidylinositol 4,5-bisphosphate) molecules, which binds to the catalytic subunit of PKC $\alpha$. The resulting activation complex is regulated by the phosphorylation status of SDC4 cytoplasmic Ser179 (human Ser179, rat Ser183) [86], which alters the conformation of the $\mathrm{C} 2$ region of the cytoplasmic domain, leading to loss of PIP2 binding and thus to a lack of PKC $\alpha$ activation [87,88]. PKC $\alpha$ is a $\mathrm{Ca}^{2+}$-dependent conventional PKC isoform, but its activation through SDC4 makes it independent of changes in $\mathrm{Ca}^{2+}$ levels and thus active in the presence of EDTA (ethylenediaminetetraacetic acid) [89]. SDC4 also establishes contact with the cytoskeleton of actin, as its cytoplasmic domain binds to alpha-actinin, a cross-linking protein between actin filaments [68]. 


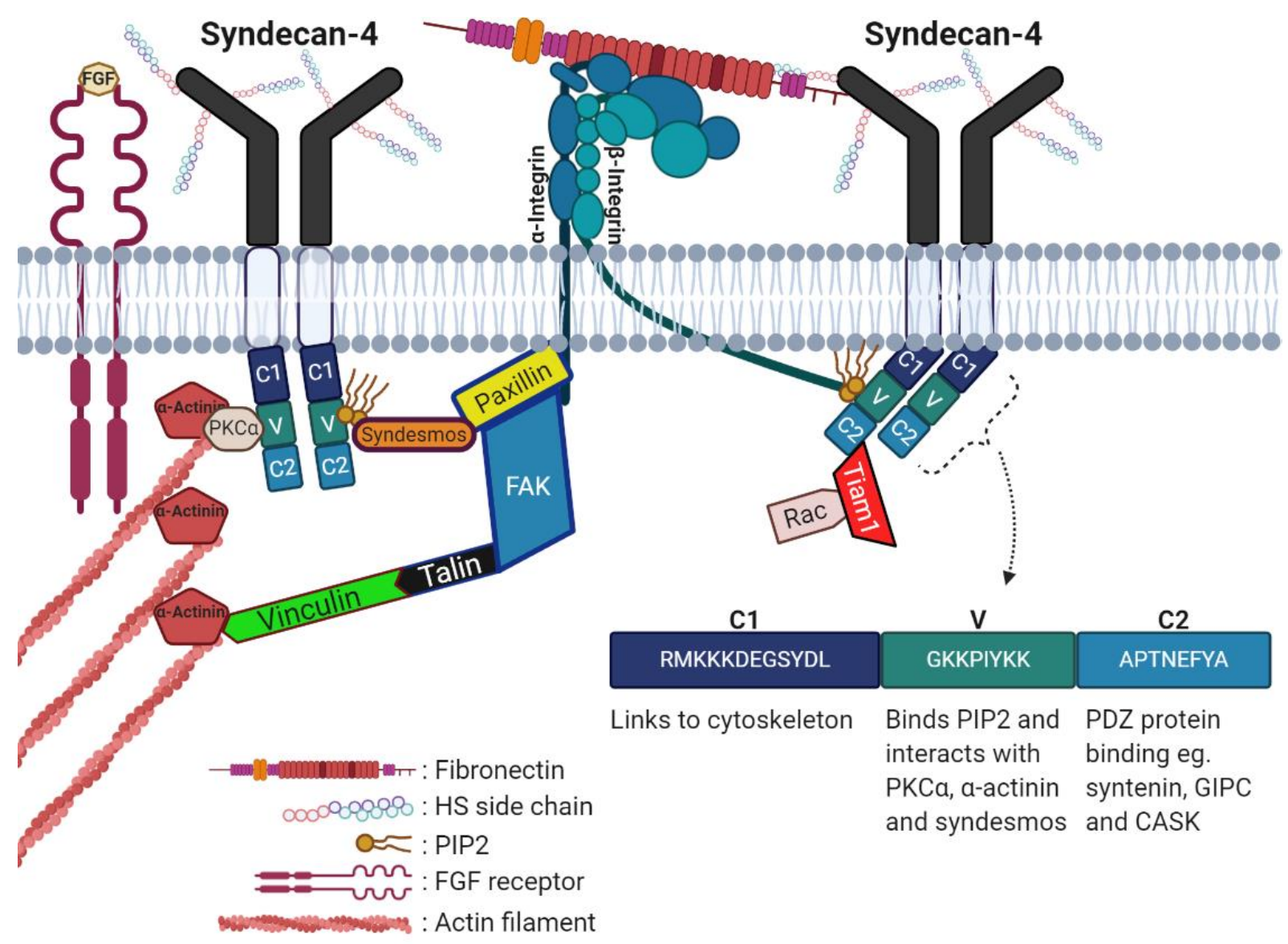

Figure 3. Structure and potential interacting partners of SDC4. Schematic representation of the SDC4 molecule showing the different domains and their possible interfering molecules, as well as the amino acid sequence of the cytoplasmic domain of the SDC4 - V variable region surrounded by the C1 and C2 constant regions. [90-92]. Image created by BioRender app.

Tiam1 interacts with SDC4 to affect cell migration, cell matrix, and cell-cell adhesions through rearrangement of the actin cytoskeleton. Tiam1 regulates actin polymerization, the rearrangement of the actin cytoskeleton, through its interaction with the Arp2/3 complex [34]. SDC4 influences matrix-induced activation of Rac1 via PKC $\alpha$ and concentrates active Rac1 and the formation of membrane protrusions on the leading side of the migrating cell $[36,93]$. The interaction between Tiam1 and SDCs has been studied previously. The affinity and strength of the interaction of different SDCs with the Tiam1 molecule are different $[94,95]$, the direct interaction of SDC4 and Tiam1 has been previously demonstrated [96]. As a result of Tiam1 binding, SDC4 regulates Rac1 activity in a Ser179 phosphorylation-dependent manner [96].

SDC4 may play a major role in the regulation of intracellular $\mathrm{Ca}^{2+}$ levels. SDC4 regulate transient receptor potential canonical (TRPCs) channels too, to control cytosolic $\mathrm{Ca}^{2+}$ equilibria, thus consequent cell behavior. SDC4 can recruit cytoplasmic PKC $\alpha$ to target serine714 of TRPC7 increasing intracellular $\mathrm{Ca}^{2+}$ concentration with subsequent control of the 
cytoskeleton in fibroblasts [97]. However, a direct interaction has not been reported between SDC4 and TRPC7 [91]. In contrast, in podocytes, SDC4 knockdown reduced the cell surface expression of the TRPC6 channel and reduced the $\mathrm{Ca}^{2+}$ concentration [98]. Furthermore, knocking down of SDC4 in HaCaT keratinocytes did not affect the $\mathrm{Ca}^{2+}$ concentration, whereas the silencing of both SDC1 and SDC4 decreased the intracellular $\mathrm{Ca}^{2+}$ by modulating TRPC4 channels [97].

\section{7. $\quad$ Results of experiments with SDC knockout mice}

Although heparan sulfate is an essential glycosaminoglycan for cellular life, SDC1 and SDC4-null mice are fertile and viable. In the absence of SDC1 and SDC4 proteoglycans, mice respond less favorably to postnatal and injury stress situations and have prolonged wound healing $[80,99,100]$. In the absence of SDC1, epithelial regeneration is slowed and the adhesion of white blood cells to the endothelium is altered. Both nutritional and learning anomalies have been detected in SDC3 gene knockout mice [101]. SDC4 has an essential role in the development and regeneration of skeletal muscle [102]. In SDC4 knockout mice, angiogenesis of granulation tissue is impaired [103,104] and skeletal muscle regeneration does not occur, with satellite cell activation and proliferation, decreased MyoD expression, myoblast fusion and differentiation [102], but the exact mechanism of these phenomena is unknown. 


\section{GAPS IN CURRENT KNOWLEDGE: AIMS OF THE THESIS}

(i) SDC4 knockout mice suffer from impaired skeletal muscle regeneration. Although the essential role of SDC4 in the development and regeneration of skeletal muscle was already described, the exact mechanism of the phenomenon is unknown. The first aim of this thesis is to look into the function of SDC4 in the migration of myoblasts.

(ii) The study of cell migration on a molecular level has exploded in popularity in recent years. The most important regulatory molecules were discovered, as well as the underlying mechanisms. However, many issues remain unresolved. Since SDC4 has not yet been related to changes in actin nanostructure, my next aim is to use the dSTORM technique to investigate the nanoscale changes in actin as a result of SDC4 knockdown. In addition, I investigate the effects of SDC4 silencing on focal adhesions of myoblasts throughout the thesis.

(iii) The polarization of cells in form, molecule, and organelles is an important process during migration. The intracellular location of cell organelles along the front-rear axis is one indicator of cellular polarization. Centrosome position on the leading edge-nucleus-trailing edge axis is a well-quantified and long-used method for determining cell polarization. My next aim in this study is to determine the position of centrosomes in order to investigate the shift in cell polarization caused by SDC4 knockdown. I will also look at SDC4's intracellular distribution.

(iv) Intracellular $\mathrm{Ca}^{2+}$ is essential for cell migration. $\mathrm{Ca}^{2+}$ influx from the extracellular space, as well as $\mathrm{Ca}^{2+}$ release from intracellular stores, both contribute to cytosolic $\mathrm{Ca}^{2+}$ concentration. Migrating cells generate a front-to-back $\mathrm{Ca}^{2+}$ gradient, which serves as a crucial coordinator for polarized signaling events. The purpose of this study is to look into the distribution of intracellular $\mathrm{Ca}^{2+}$ in migrating myoblasts following SDC4 knockdown.

(v) It is well understood that in SDC4 knockout cells, the amount of delocalized, activated Rac1 increases over time. Furthermore, Tiam1 has been identified as a GEF that functions as a specific Rac1 activator and is involved in biological processes such as cell migration and polarization. My further goal was to study whether Rac1 inhibition ameliorates the effect of SDC4 knockdown on migration and whether Tiam1 expression and localization are affected by SDC4. 


\section{MATERIALS AND METHODS}

\subsection{Cell culture and plasmids}

C2C12 mouse myoblast (ATCC; Manassas, VA, USA) cultures were grown in medium containing 80\% high-glucose Dulbecco's modified Eagle's medium (containing $4.5 \mathrm{~g} / \mathrm{L}$ glucose $584 \mathrm{mg} / \mathrm{L}$ glutamine and $110 \mathrm{mg} / \mathrm{L}$ pyruvate; Corning, New York, NY, USA), 20\% FBS (fetal bovine serum) (Gibco/Thermo Fisher Scientific, Waltham, MA, USA), and 65 $\mu \mathrm{g} / \mathrm{mL}$ gentamicin (Lonza, Basel, Switzerland). Cells were transfected stably using shRNA expressing plasmids (OriGene, TR513122; Rockville, MD, USA) and X-tremeGENE transfection reagent (Roche, Basel, Switzerland).

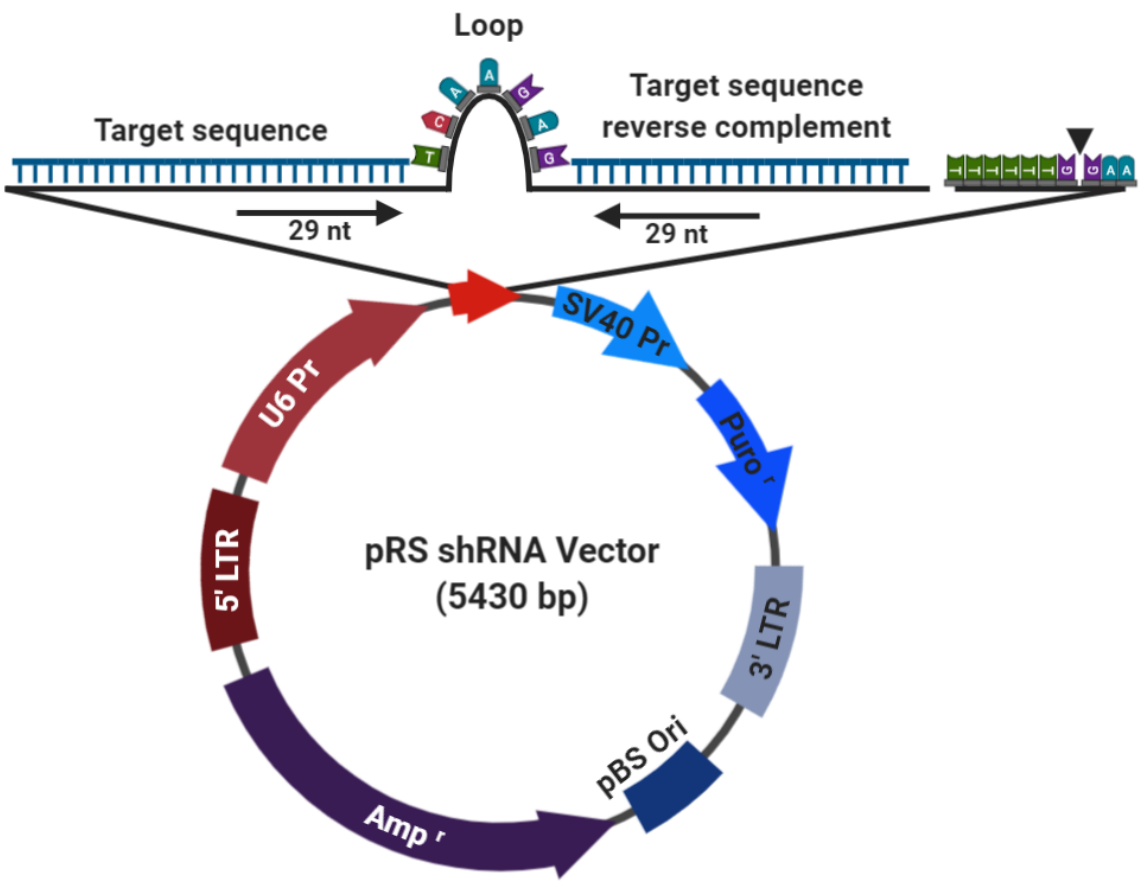

Figure 4. pRS vector. The image is created by BioRender app.

For SDC4 silencing, pRS shRNA vectors (fig. 4.) were applied targeting the following sequences: 5'-GAA CTG GAA GAG AAT GAG GTC ATT CCT AA-3' (shSDC4\#1) or 5'GCG GCG TGG TAG GCA TCC TCT TTG CCG TT-3' (shSDC4\#2). The scrambled plasmid targeted the 5'-GCA CTA CCA GAG CTA ACT CAG ATA GTA CT-3' sequence. Selection of the transfected cells was carried out by adding $4 \mu \mathrm{g} / \mathrm{mL}$ puromycin (SigmaAldrich, St. Louis, MO, USA) to the medium.

\subsection{Western blotting}

Non-transfected, scrambled and SDC4 knockdown (shSDC4\#1 and shSDC4\#2) cells were harvested in RIPA buffer (20 mM Tris- $\mathrm{HCl}$ pH 7.5, $150 \mathrm{mM} \mathrm{NaCl}, 1 \mathrm{mM}$ Na2EDTA, 1 
mM EGTA, $1 \%$ NP-40, $1 \%$ sodium deoxycholate, $2.5 \mathrm{mM}$ sodium pyrophosphate, $1 \mathrm{mM}$ glycerophosphate, $1 \mathrm{mM}$ Na3VO4, $1 \mu \mathrm{g} \times \mathrm{ml}$ leupeptin; Cell Signaling Technology, Danvers, MA, USA; \#9806) supplemented with $1 \mathrm{mM}$ Sodium-fluoride and protease inhibitor cocktail (Sigma-Aldrich). The samples were spun down at $18,927 \times \mathrm{g}$ for $5 \mathrm{~min}$ at $4{ }^{\circ} \mathrm{C}$ to eliminate cellular debris and the supernatants were separated by SDS/PAGE and blotted to nitrocellulose membrane. After blocking, membranes were incubated with primary antibodies including rabbit anti-SDC4 (PA1-32485; Pierce Protein Biology - Thermo Fisher Scientific, Waltham, Massachusetts, USA) and mouse anti-GAPDH (Cell Signaling Technology, \#2118, Danvers, Massachusetts, US). Following incubation with the appropriate horse-radish peroxidase-conjugated anti-IgG secondary antibodies (DAKO, Glostrup, Denmark), peroxidase activity was visualized by enhanced chemiluminescent method (Advansta, Menlo Park, CA, USA). Quantification of signal intensity was carried out by QUANTITY ONE software (Bio-Rad, Hercules, CA, USA).

\subsection{Cell migration assays}

Two layouts were used to perform the experiments and to investigate the intracellular processes that occur during cell migration: random- and directional migration assays, both in two dimensions (fig. 6. A, B).

For random migration, cells were plated (on 6-, 12- or 24-well plates), allowed to adhere to the surface, then the proliferating medium was replaced with a medium containing reduced serum to avoid cell division and proliferation. The optimal number of cells were $3 \times 10^{4} /$ well in a 6-well plate, $1.5 \times 10^{4} /$ well in a 12 -well plate and $7.5 \times 10^{3} /$ well in a 24 -well plate.

A cell-free zone or wound presenting the lesion or injury can be formed on a confluent cell culture by scratching with a pipette tip (wound scratch assay; fig. 5. A). In the case of directional migration, a larger number of cells is recommended for plating $\left(1,8 \times 10^{5}\right.$ cells / well on a 6-well plate for wound scratch assay), since then confluent cell culture must be occupied to represent in vivo environment of wound healing. Once the cells have adhered to the surface, a medium with reduced serum content is used, followed by scratching the culture with a pipette tip to create a cell-free zone (so called "wound"). Studies can examine the "healing" of this "wound". Cells at the edge of the cell-free zone are more prone to migration, while cells inside the confluent cell culture are less motile due to the physical barrier of the cells in front of them. Cells in both the first, second and subsequent rows can be examined for migration, intracellular processes and so on. 
A

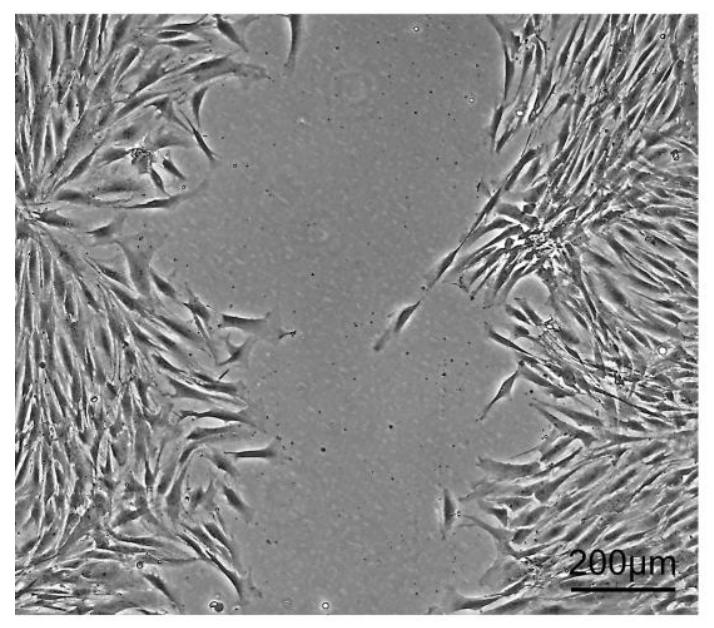

B

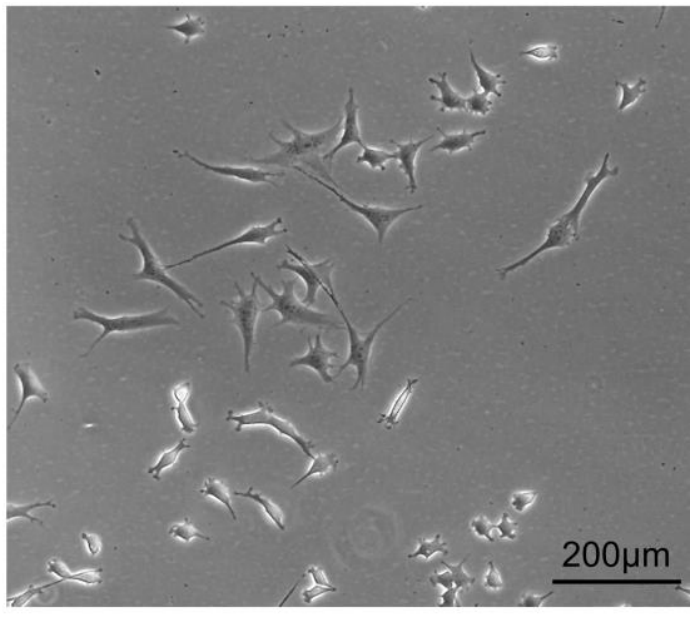

Figure 5. Representative images of migrating cell during different migration assays. Cell migration during wound scratch assay $(A)$ and random migrating cells $(B)$.

A two-dimensional directional migration assay can also be performed with silicone inserts (ibidi GmbH, Gräfelfing, Germany), which were also used in this work to provide a defined, regular-edged cell-free area during use, as the two sides of the inserts are separated by a partition wall to provide the cell-free area (fig. 5. B). The inserts were placed in the wells of 6-well cell culture dishes, and $3 \times 10^{4}$ cells in both wells of the inserts were plated. After 1 $\mathrm{h}$ of incubation in proliferation medium, the medium was replaced with serum-reduced one to eliminate cell division. Cells were kept in this serum-reduced medium for $24 \mathrm{~h}$, followed by removal of the insert and examination of migration.

A

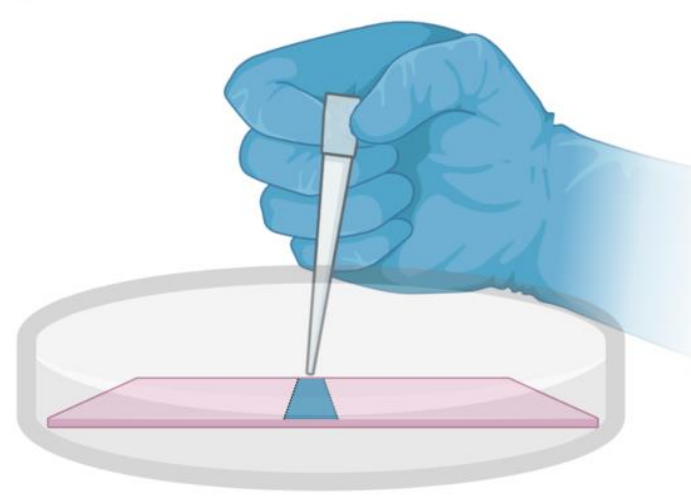

B



Figure 6. Schematic illustration of the implementation of in vitro wound healing assay (scratch assay) (A) and ibidi silicon inserts (B).

\subsection{Time-lapse imaging of the migration of live cells}

For random migration assays, cells were seeded in 24-well plates at a density of $7.5 \times 10^{3}$ cells/well. After 60 minutes, the medium was changed to a serum-reduced one to suppress cell 
division and $24 \mathrm{~h}$ later the nuclei were stained with Hoechst33342 (1:2000, $1 \mathrm{mg} / \mathrm{mL}$ stock solution; Sigma-Aldrich) and Rac1 was inhibited by NSC23766 treatment during the measurement (50 $\mu \mathrm{M}$; Tocris Bioscience, Bristol, United Kingdom).

In the case of two-dimensional directional migration, cells were seeded into the reservoirs of 2-well cell culture silicon inserts at a density of $3 \times 10^{4}$ cells/well (Ibidi, Martinsried, Germany). Upon cellular attachment, the medium was replaced with serumreduced medium, and nuclei were stained as described above and after washing with PBS, the insert was removed and the migration of cells into the cell-free zone was screened.

Time-lapse images were captured in 20 min intervals for $8 \mathrm{~h}$ in directional-, and for $18 \mathrm{~h}$ in random migration assay at $37^{\circ} \mathrm{C}$ and $5 \% \mathrm{CO} 2$ using the PerkinElmer Operetta (PerkinElmer, Inc., Waltham, MA, United States) high-content imaging system with a $20 \times$ objective $(20 \times$ long WD; NA $=0.45$, working distance: $7.8 \mathrm{~mm}$; field of view: $675 \times 509$; depth of focus: $4.6 \mu \mathrm{m}$; optical xy resolution: $0.7 \mu \mathrm{m}$ ).

\section{5. $\quad$ Single-cell tracking of cultured myoblasts}

To evaluate the results obtained during time-lapse microscopy, the cell nuclei were tracked frame-by-frame with ImageJ (National Institutes of Health, Bethesda, MD, United States) and CellTracker [105] software. CellTracker is an online, accessible freeware based on MATLAB with high efficiency to evaluate cell migration (http://www.celltracker.website/index.html). Dying or damaged and possibly dividing cells were excluded from the evaluation process.

In both the directional and random migration assays, the length of total path, maximum distance from the origin, as well as the average and maximum cell speeds were calculated. The vectorial distance of migration (i.e., real shift of the cell) from the origin was also quantified. The individual migratory tracks of the cells into the cell free zone was visualized. To illustrate the migratory trajectories of randomly migrating cells, two-dimensional windrose plots were created using Excel DiPer Plot_At_Origin macro [106] by transposing the $\mathrm{x}$ and y coordinates of the cell tracking data to a common origin.

The directionality of the cell movement was described by the persistence index, which was defined by the ratio of the vectorial distance (the distance between the origin and the endpoint of the movement) and the length of total path. 


\subsection{Wound scratch assay}

For the wound scratch assay, cells were grown in 6-well plates until they reached confluence. After $24 \mathrm{~h}$ incubation in serum-reduced medium, cell-free zones were created by scratching the cell layer with a P200 pipette tip. Images of the cell-free zone were captured immediately (0h), 4 and $8 \mathrm{~h}$ after wounding, using a Leica DMi1 phase-contrast microscope (Leica Microsystems, Wetzlar, Germany). Between imaging periods, the cells were incubated at $37{ }^{\circ} \mathrm{C}$ and $5 \% \mathrm{CO} 2$. The area of the cell-free zone was measured using Digimizer image analysis software (MedCalc Software bvba, Ostend, Belgium). The closure of the cell-free area was calculated as follows: (area of cell-free zone at $\mathrm{t} \mathrm{O}_{\mathrm{h}}-$ area of cell-free zone at $\mathrm{tx}_{\mathrm{h}}$ ) / area of cell-free zone at $\mathrm{t} 0_{\mathrm{h}}$.

\section{7. $\quad$ Fluorescence staining}

For fluorescence staining, after one h of incubation, cells were seeded for $24 \mathrm{~h}$ onto FBS (Gibco/Thermo Fisher Scientific, Waltham, MA, USA) coated glass coverslips (Hirschmann Laborgeräte GmbH \& Co. KG, Eberstadt, Germany).

For Tiam1 staining, cells were fixed with 4\% paraformaldehyde (Molar Chemicals Kft., Halásztelek, Hungary) for $10 \mathrm{~min}$, permeabilized with 0.3\% Tween 20 (Sigma-Aldrich, Inc., St. Louis, Missouri, USA), and blocked with 1\% bovine serum albumin (Sigma-Aldrich) in PBS at room temperature. Rabbit polyclonal anti-Tiam1 primary antibody (OST00085W; Invitrogen, Carlsbad, CA, USA) was visualized with the appropriate Alexa488-conjugated secondary antibody (Invitrogen). Wide-field fluorescence images were obtained on a Nikon Eclipse Ti-E microscope (Nikon Instruments Inc., 1300 Walt Whitman Road Melville, NY 11747-3064, USA) with $40 \times$ objectives.

For centrosome staining, cells were fixed with methanol 2, 4, and $6 \mathrm{~h}$ after wounding the confluent culture. After permeabilization with 0.5\% Tween-20 (Sigma-Aldrich), the samples were blocked in $4 \%$ bovine serum albumin (BSA; Sigma-Aldrich), and stained with a mouse monoclonal anti- $\gamma$-tubulin antibody (1:200; Sigma-Aldrich) at $4{ }^{\circ} \mathrm{C}$ overnight, followed by incubation with an Alexa Fluor 488-conjugated anti-mouse secondary antibody (Jackson ImmunoResearch, Cambridgeshire, United Kingdom) a day later.

To visualize the actin filaments, cells subjected to the above-described scratch assay were fixed with a methanol-free $4 \%$ formaldehyde solution (Thermo Fischer Scientific) $2 \mathrm{~h}$ after wounding. After permeabilization with $0.3 \%$ Triton X-100 (Sigma-Aldrich) and blocking in 4\% BSA (Sigma-Aldrich), the actin filaments were stained with Alexa Fluor 647conjugated phalloidin (A22287, Thermo Fisher Scientific). 
For SDC4, anti-FAK (anti-focal adhesion kinase) and anti-GM130 (anti- Golgi matrix protein of $130 \mathrm{kDa}$ ) immunostaining, myoblasts were fixed with $4 \%$ formaldehyde solution 2 $\mathrm{h}$ after wounding, permeabilized with $0.3 \%$ Triton X-100 and blocked with $1 \%$ BSA. Rabbit polyclonal anti-SDC4 primary antibody (immunogen: synthetic peptide surrounding amino acid 184 of human SDC4; PA1-32485; Invitrogen, Carlsbad, CA, United States) was visualized with the appropriate Alexa Fluor 568-conjugated (Invitrogen), or Alexa Fluor 488conjugated secondary antibody (Jackson ImmunoResearch, Cambridgeshire, United Kingdom). Focal adhesions were marked with mouse monoclonal anti-FAK primary antibody (sc-271126; Santa Cruz Biotechnology, Dallas, TX, United States) and with Alexa Fluor 488conjugated secondary antibody (Jackson ImmunoResearch, Cambridgeshire, United Kingdom). The cis-Golgi network was stained by mouse monoclonal anti-GM130 antibody (610822; BD Biosciences, San Jose, CA, United States) and followed by incubation with CF568-conjugated secondary antibody (Biotinum, Fremont, CA, United States). Nuclei were counterstained with Hoechst 33258 (0.01 mg/mL, Sigma-Aldrich).

\subsection{Evaluation of the Tiam1 distribution}

The wide-field fluorescence images were recolored as heat maps using ImageJ (National Institutes of Health, Bethesda, MD, USA, https://imagej.nih.gov/ij/) image analysis program (Image>Lookup Tables). Each pixel was colored based on the pixel intensity value (16-bit images; 0-65535 gray value) according to the scale bar shown in the images (the lowest intensity pixels are shown as blue; the highest intensity pixels are shown as red). The contours of the individual cells were drawn (Analyze>Analyze particles) and the average pixel intensity within the border of the cells were quantified (Analyze>Measure) in the different cell lines. The intensity value of each pixel was measured within the selected area and the sum of the intensities was divided by the area of the cell to obtain the average Tiam1 intensity value of the individual cells. To measure the amount of variation in the pixel intensities, the standard deviation of the intensity values was also calculated in every cell (Analyze>Measure).

\subsection{Determining and quantifying the position of centrosomes}

The positions of centrosomes were analyzed to quantify cell polarity, based on a previous characterization of centrosome reorientation in response to a scratch [107]. Anti- $\gamma$-tubulinstained samples were inspected and imaged using a Nikon Eclipse Ti-E microscope frame (Nikon Instruments Inc., Melville, NY, United States) with epifluorescent illumination using 
$20 \times$ objective (Nikon Plan fluor $20 \times$ DIC N2, NA $=0.50$ ). The images were analyzed using ImageJ software. Two h after wounding, only the migrating cells next to the scratched area were analyzed. For selected cells adjacent to the cell-free zone, the direction of migration was designated as perpendicular to the wound edge, the nucleus was set as the origin and a $30^{\circ}$ circular sector facing the direction of wound closure was assigned. Centrosomes situated within this assigned area were scored as correctly oriented. To monitor the time dependency of centrosome reorientation in different cell lines, the position of centrosomes was analyzed 2 , 4 and $6 \mathrm{~h}$ after wounding in the 1st and 2nd row of myoblasts in the different cell lines along the wound edge based on the method described by Gotlieb et al. (1983). The position of centrosomes was considered "toward" the wound edge (between the nucleus and the wound edge), "middle" (along the side the nucleus), or "away" (between the nucleus the monolayer behind the cells).

\subsection{Evaluation of SDC4 immunostaining}

Wide-field fluorescence images of SDC4 immunostained samples were acquired by a Nikon Eclipse Ti-E microscope (Nikon Instruments Inc.) with $40 \times($ Nikon CFI Plan Fluor 40 $\times, \mathrm{NA}=0.75)$ and $100 \times($ Nikon CFI Plan Apo DM Lambda $100 \times$ Oil, NA $=1.45)$ objectives and pseudo-colored using ImageJ. The contours of the individual cells were drawn and the average pixel intensity within the border of the cells were quantified following background correction. The intensity value of each pixel was measured within the selected area and the sum of the intensities was divided by the area of the cell to obtain the average SDC4 intensity value of the individual cells. Furthermore, cells were partitioned into 4 quadrants considering the nucleus as the origin, a $90^{\circ}$ circular sector facing the direction of the wound closure was assigned and the SDC4 signal intensity within this area was quantified.

\subsection{Quantification of focal adhesions}

Wide-field fluorescence images of FAK immunostained samples were acquired by a Nikon Eclipse Ti-E microscope (Nikon Instruments Inc.) with a $100 \times($ Nikon CFI Plan Apo DM Lambda $100 \times$ Oil, NA $=1.45)$ objective and processed using ImageJ software. The images were converted into binary images, particles in the range of 0 to $100 \mu \mathrm{m} 2$ were selected. The masks of these selected particles were constructed and the area of each particle was measured. 


\subsection{Super-resolution dSTORM imaging}

Super-resolution direct stochastic optical reconstruction microscopy (dSTORM) measurements of phalloidin-stained samples were performed using a custom-made inverted microscope based on a Nikon Eclipse Ti-E frame. After conditioning (through spatial filtering via fiber coupling and beam expansion), the applied laser beams were focused into the back focal plane of the microscope objective (Nikon CFI Apo $100 \times, \mathrm{NA}=1.49$ ) to produce a collimated beam on the sample. The angle of illumination was then set through a tilting mirror mounted into a motorized gimbal holder and placed into the conjugate plane of the sample. All dSTORM images were captured under epi-illumination at an excitation wavelength of 634 nm (Thorlabs HL63133DG: $637 \mathrm{~nm}$, Pmax $=170 \mathrm{~mW}$ in a Thorlabs TCLDM9 TE-Cooled mount set to $19^{\circ} \mathrm{C}$ ). The laser intensity was controlled via a Thorlabs LDC500 laser driver and set to an output of 2-4 kW/cm2 on the sample plane. An additional laser (Nichia: $405 \mathrm{~nm}$, Pmax $=60 \mathrm{~mW}$ ) was used for reactivation. Images were captured using an Andor iXon3 897 BV EMCCD digital camera (512 pixels $\times 512$ pixels; pixel size: $16 \mu \mathrm{m})$. The size of the illuminated sample region was matched to the size of the detector, which determined the field of view $(80 \times 80 \mu \mathrm{m} 2)$. Typically, the frame stacks for dSTORM super-resolution images were captured at a reduced image size (i.e., crop mode). A fluorescence filter set (Semrock, LF405/488/561/635-A-000 dichroic mirror with a BLP01-647R-25 emission filter) was used to select and separate the excitation and emission lights in the microscope. During measurements, the perfect focus system of the microscope was used to maintain focus on the sample at a precision level of $<30 \mathrm{~nm}$. Immediately before measurement, the sample storage buffer was replaced with a GLOX switching buffer [108] and the sample was mounted on a microscope slide. During a typical imaging session, 20,000 frames were captured at an exposure time of 20 or $30 \mathrm{~ms}$. The image stacks were analyzed using rainSTORM localization software [109] and reconstructed using the built-in Simple Histogram method with a superpixel size of $13.33 \mathrm{~nm}$. The Thompson-precision [110] and PSF size acceptance ranges were set to $0-35 \mathrm{~nm}$ and $0.7-1.5$ pixels, respectively.

\subsection{Evaluation of dSTORM images}

After dSTORM imaging, phalloidin-stained samples were subjected to a nanoscale analysis of the actin cytoskeleton. The dSTORM images of lamellipodial actin structures were processed using ImageJ software. The super-resolution images were converted to grayscale, adjusted to a fixed threshold and noise filtered. The ImageJ Skeletonize function was used to create binary skeletonized images. Then the Skeleton Analysis plugin was used to calculate 
the number of branches belonging to each skeleton in every image and to measure the length of each individual branch. To describe the difference between the cortical actin-rich region and the inner actin-depleted area of the lamellipodial actin network, three areas (each $126 \times$ $124 \mathrm{px}$ ) were randomly selected in the external region (with a width of $350 \mathrm{px}$ beneath the plasma membrane) and three in the inner, internal region of the lamellipodia. Then the average number of branches and average length of the individual branches were measured in each of these selected rectangles and compared.

\subsection{Assessment of intracellular $\mathrm{Ca}^{2+}$ distribution}

As control, scrambled and two SDC4-targeted myoblast cell lines were seeded onto glass 8-well chambered coverslips (ibidi $\mathrm{GmbH}$, Gräfelfing, Germany) at $1 \times 10^{4}$ cells/well density and grown for $24 \mathrm{~h}$ in serum-reduced medium. The confluent cultures were scratched as described above and further incubated for $2 \mathrm{~h}$. Subsequently, the cells were subjected to $2 \mu \mathrm{M}$ Fluo-4 AM and $3 \mu \mathrm{M}$ Fura Red AM (Thermo Fisher Scientific) in serum-free D-MEM containing $50 \mu \mathrm{M}$ Verapamil (Sigma) for $30 \mathrm{~min}$ at $37^{\circ} \mathrm{C}$ and $5 \% \mathrm{CO}$. Verapamil was included to block the activity of multidrug transporters hindering effective dye loading. After several thorough washing steps, the green (493-572 nm) and far red (609-797 $\mathrm{nm}$ ) fluorescence images were simultaneously acquired at 488 and $458 \mathrm{~nm}$ excitations, respectively, using a Zeiss 710 LSM laser scanning fluorescence confocal microscope with a Plan-Apochromat $40 \times($ N.A. $=1.4)$ oil immersion objective. The images were analyzed by ImageJ 1.49g software (National Institutes of Health, Bethesda, MD, United States). Ratio images were generated using the Ratio Plus Plug-in. For quantitative analysis, the Fluo-4 and Fura Red fluorescence intensities were determined along the axis of migrating cells starting from the leading edge. After background correction, ratios of green and red fluorescence were calculated. The slope of the intracellular $\mathrm{Ca}^{2+}$ distribution was determined by least squares method.

\subsection{Statistical analysis}

Differences between groups were analyzed using a one-way ANOVA, followed by the Dunett and Scheffe post hoc test or Student's t-test. GraphPad Prism 7.0 (GraphPad Software Inc., San Diego, CA, United States) was used for graphing and statistical analyses. The data are expressed as means + standard errors of the means. A $p<0.05$ was considered significantly different. 


\section{RESULTS}

\subsection{SDC4 silencing reduces myoblast migration}

SDC4 expression was monitored in $\mathrm{C} 2 \mathrm{C} 12$ myoblasts stably transfected with plasmids expressing SDC4-specific shRNA (shSDC4\#1 and SDC4\#2 cell lines) by Western blotting. I observed a more significant decrease in SDC4 expression in shSDC4\#1 than shSDC4\#2 cells. A scrambled sequence was applied as a control and had no effect on SDC4 expression (fig. 7. A, B.).

A

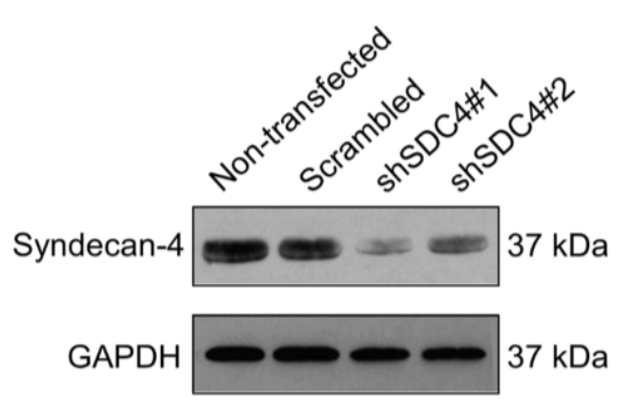

B

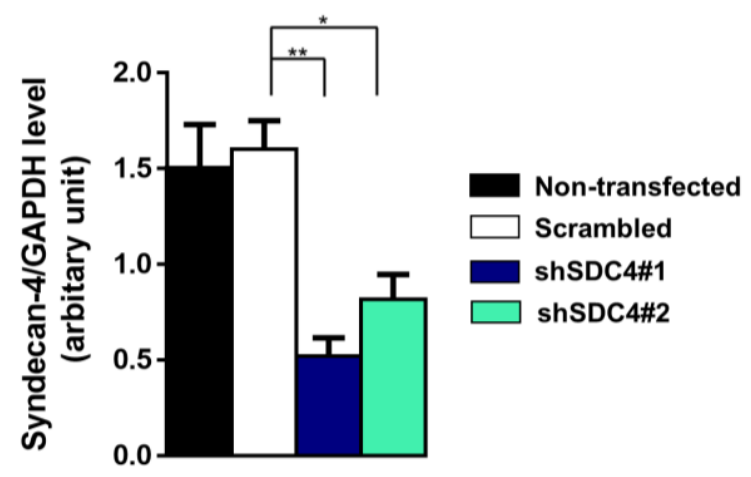

Figure 7. Effect of SDC4 silencing on C2C12 myoblasts. (A) Representative Western blot analysis depicts the level of SDC4 in cells stably expressing shRNA against SDC4 (shSDC4\#1, shSDC4\#2), or scrambled shRNA. Equal protein loading was monitored by GAPDH. (B) Quantification of SDC4 expression in the different cell lines. $n=6$ independent experiments, means + standard errors of the means; **: $p<0.01 ; *: p<0.05$.

Cell migration normally relies on a variety of external signals, such as chemical, mechanical, or electrical, which instruct cells in which direction to move. The question of whether in vitro directional and random migration experiments are indeed compatible with in vivo processes, in vivo wound healing and in vivo cell migration has been investigated many times [111-116]. The intracellular processes that take place during in vitro cell migration can be considered valid and the basis of the processes that take place in the in vivo environment [117-120].

We then measured the effect of SDC4 knockdown on directional migration in vitro into cell-free zones created using cell culture inserts for an $8 \mathrm{~h}$ period. SDC4 knockdown significantly reduced the total path of migration, both the vectorial distance (i.e., the real shift of the cells) and the maximum distance from the origin; furthermore, the average and maximum speed were also decreased. No significant difference was observed between the non-transfected and scrambled cells. Moreover, a greater reduction was observed in migratory 
parameters in shSDC4\#1 cells, consistent with the previous observation of greater SDC4 suppression in this line.

An evaluation of the migratory tracks of individual cells depicts the positions of the $\mathrm{x}$ and $y$ coordinates corresponding to the paths taken by each cell during the indicated time (as z; fig. 8. B). The migratory tracks of highly motile control cells crossed each other in the middle of the cell-free zone (black area in the center of each image), whereas those of SDC4 knockdown cells hardly moved from the original $\mathrm{x}-\mathrm{y}$ positions during the $8 \mathrm{~h}$ experimental period. We then prepared histograms to depict the percentages of cells within each velocity range (fig. 8. C). Notably, the histograms of the non-transfected and scrambled cells formed bell-shaped curves, whereas those of both silenced cell lines exhibited a left-skewed distribution suggesting the higher ratio of less motile cells.
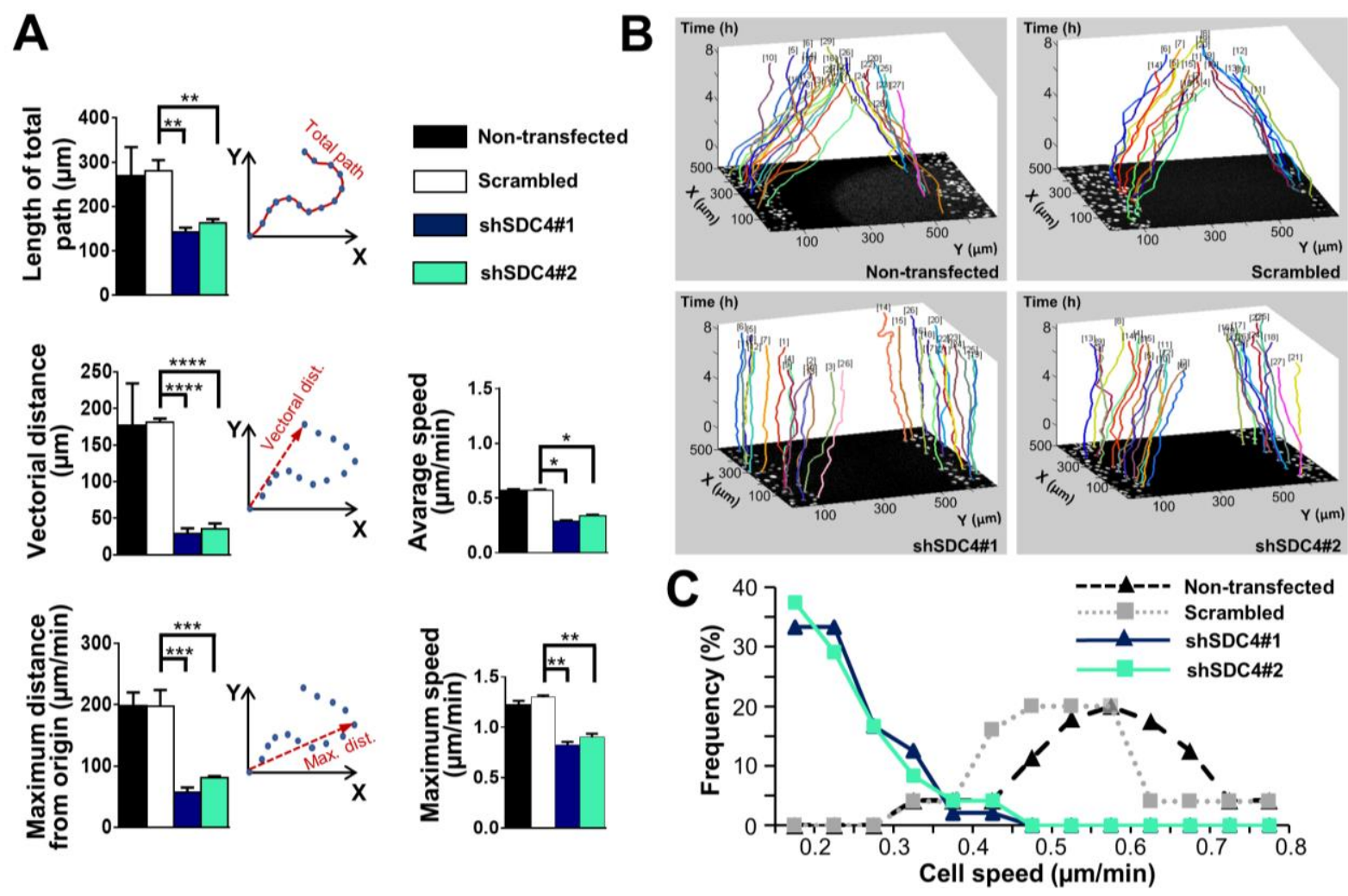

Figure 8. The role of SDC4 in the directional migration of myoblasts. (A) Migration into the cell-free zone of non-transfected, scrambled and SDC4-silenced (shSDC4\#1 and shSDC4\#2) C2C12 myoblasts was evaluated after the removal of cell culture inserts. The total length of movement, maximum distance from the starting point, vectorial distance ((i.e., real displacement of the cells), the average and maximum cell speeds during directional migration are presented. The total duration of live cell microscopy was $8 \mathrm{~h}$, at a frame rate of 3/1 h. Four independent experiments, 60-87 cells/cell line and 5-6 fields of view/experiment. Data are presented as means + standard errors of the means; $* p<0.05$, ** $p<0.01$, $* * * p<0.001$ and $* * * * p<0.0001$. (B) Representative three-dimensional migration tracks. Each color represents the complete path taken by a myoblast; $x$ and $y$ axes: position of the cell $(\mu m), z$-axis: time $(h)$. (C) Histograms depict the distributions of cells from different lines according to cell speed cells (intervals of $0.05 \mu \mathrm{m} / \mathrm{min}$ ). The frequencies of cells from each line with average speeds within each interval were evaluated and are presented on the $y$ axis $(C)$. 
Representative images (fig. 9. A) depict a scratch wound in a confluent culture at 0, 4 and $8 \mathrm{~h}$. Quantification of the wound closures revealed a reduced closure of the cell-free zone in both SDC4 knockdown lines (fig. 9. B). No significant difference was observed between non-transfected and scrambled cells (fig. 9. B).

A

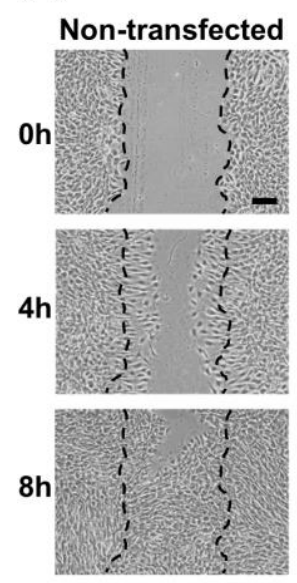

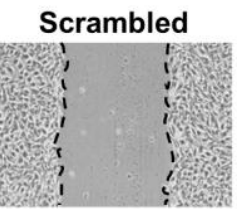
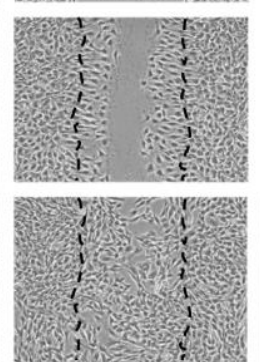
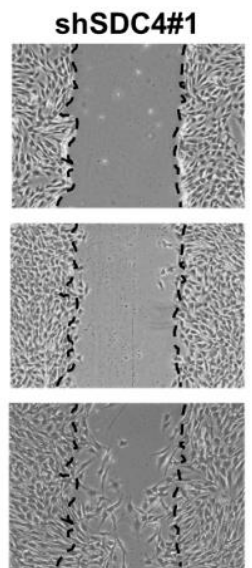

B
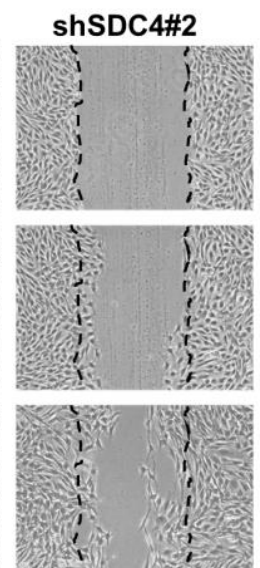
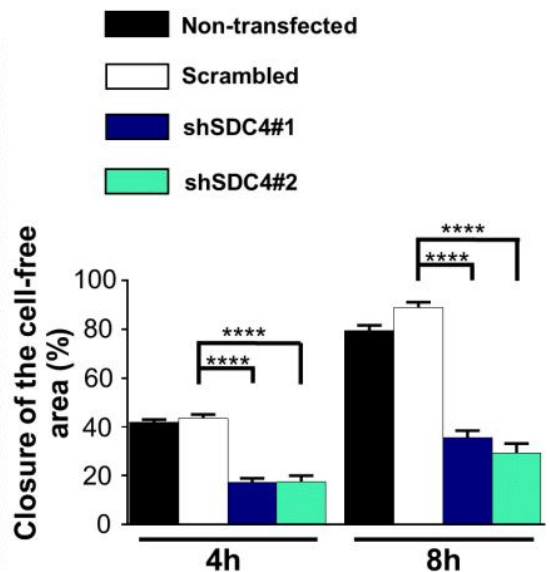

Figure 9. SDC4 knockdown delays the closure of the cell-free zone. Representative microscopy images taken 0, 4 and $8 \mathrm{~h}$ after the initiation of a wound scratch assay. Dashed lines indicate the position of the cell-free zone at 0 h. Scale bar: $200 \mu \mathrm{m}$ (A). Quantification of the closure of the cell-free area in cultures of non-transfected, scrambled and SDC4-silenced (shSDC4\#1 and shSDC4\#2) cells (B); $n=4$ independent experiments. Data are shown as means + standard errors of the means; $* * * * p<0.0001$.

\subsection{SDC4 affects the nanoscale architecture of the actin cytoskeleton, as determined by super-resolution ISTORM}

Cell motility is regulated by both extracellular factors and internal signaling mechanisms, including actin cytoskeletal remodeling. As SDC4 plays a crucial role in the organization of the actin cytoskeleton [73,121,122], thus actin filaments were evaluated using wide-field fluorescence microscopy (fig. 12. A, B, D, E, G, H, J, K) and single-molecule localization super-resolution dSTORM imaging (lower magnification: fig. 12. A, D, G, J; higher magnification: fig. 12. C, F, I, L). Notably, our super-resolution dSTORM images reveal the sub-diffraction structure of the actin cytoskeleton and enable a more sophisticated experimental comparison of control and SDC4 knockdown samples. The reduced fluorescence background and enhanced resolution enabled visualization of the orientations and densities of individual actin bundles. 
Non-transfected

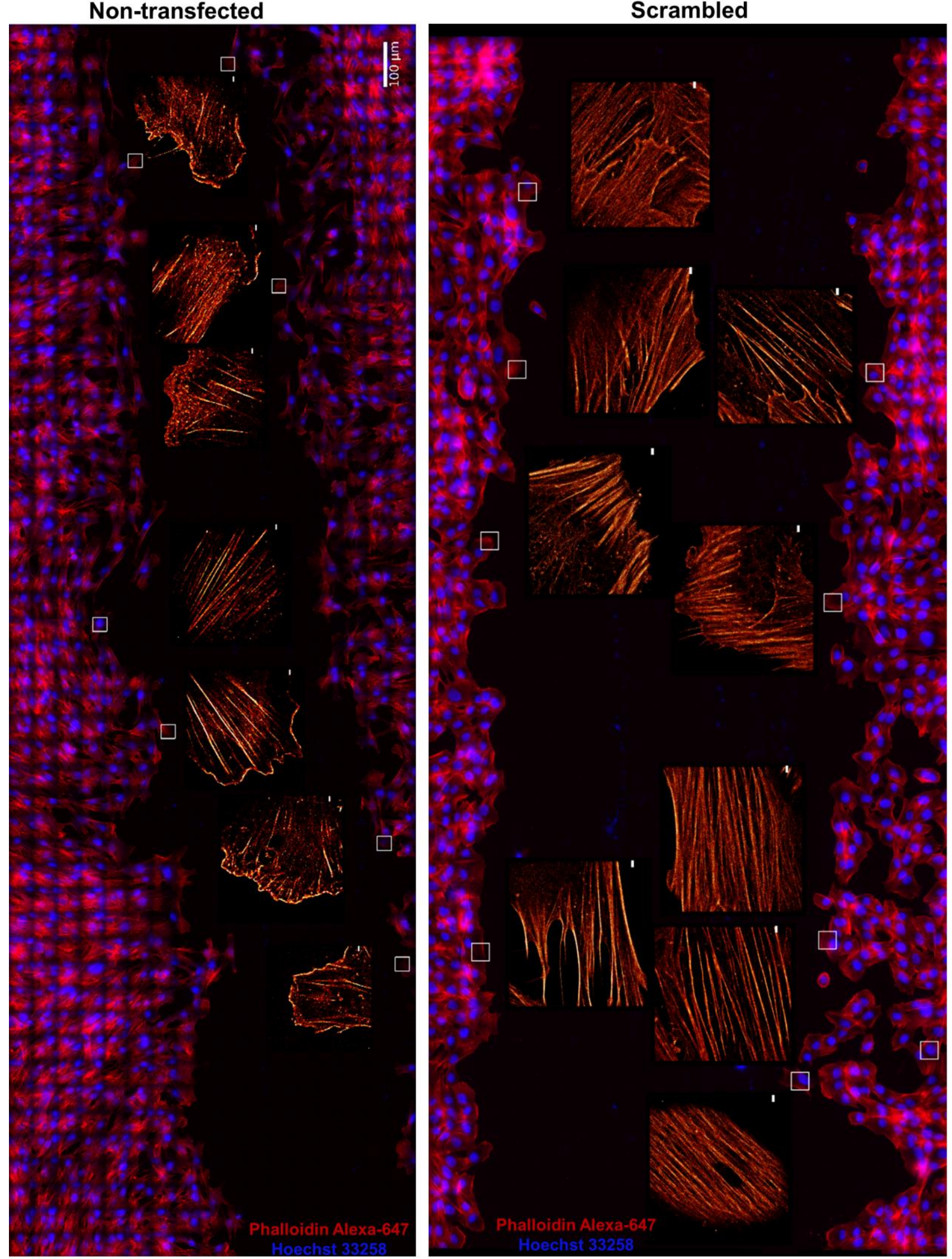

Figure 10. Actin cytoskeletal structure of non-transfected and scrambled C2C12 myoblasts around the cell-free zone. A representative wide-field fluorescence microscopy image depicts the actin skeletons of nontransfected and scrambled myoblasts around the cell-free zone. The inserts depict the lamellipodial actin structures in the migrating cells, which were imaged using dSTORM. The cells were fixed $2 \mathrm{~h}$ after confluent cultures were scratched, and the actin filaments were stained with Alexa Fluor 647-conjugated phalloidin (red). Nuclei are shown in blue (Hoechst 33258). 
shSDC4\#1

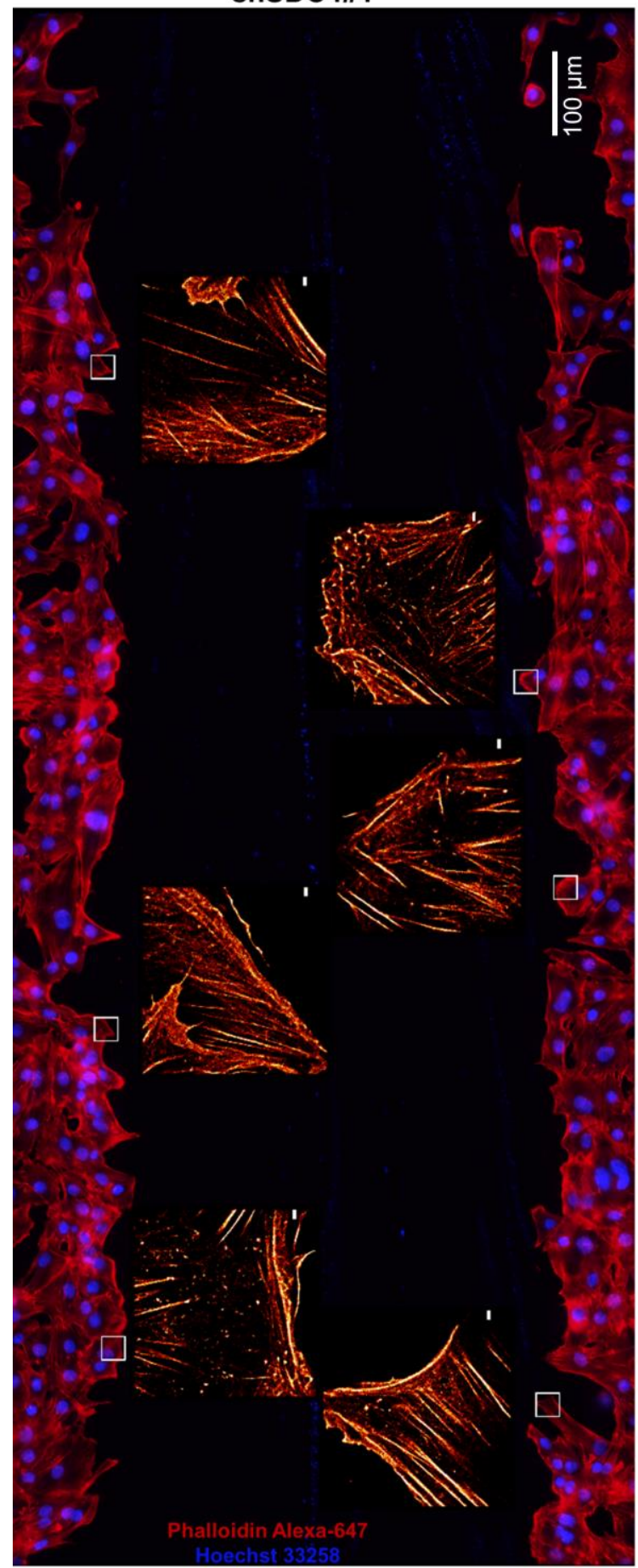

shSDC4\#2

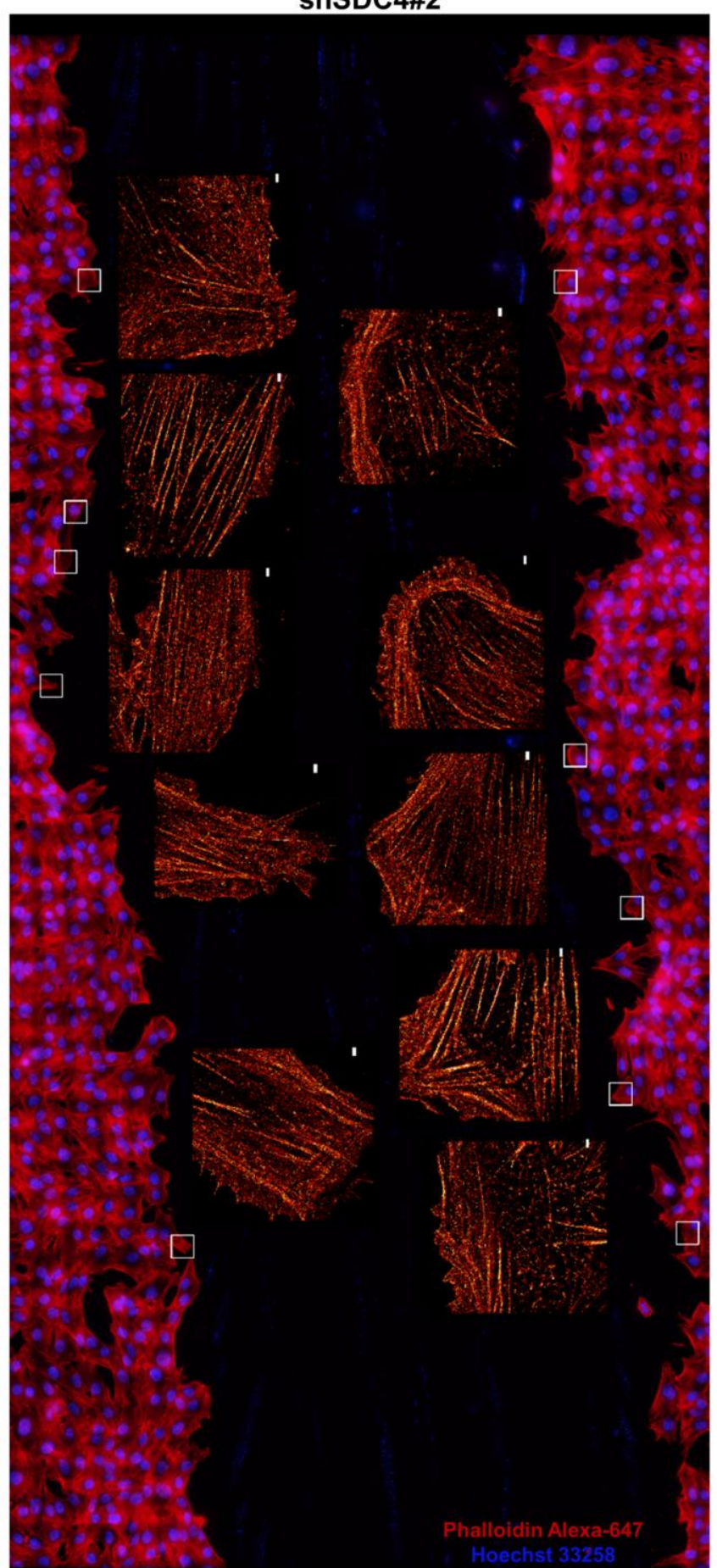

Figure 11. Actin cytoskeletal structure of shSDC4\#1and shSDC4\#2 myoblasts around the cell-free zone. A representative wide-field fluorescence microscopy image depicts the actin skeletons shSDC4\#1 and shSDC4\#2 myoblasts around the cell-free zone. The inserts depict the lamellipodial actin structures in the migrating cells, which were imaged using dSTORM. The cells were fixed $2 \mathrm{~h}$ after confluent cultures were scratched, and the actin filaments were stained with Alexa Fluor 647-conjugated phalloidin (red). Nuclei are shown in blue (Hoechst 33258). 

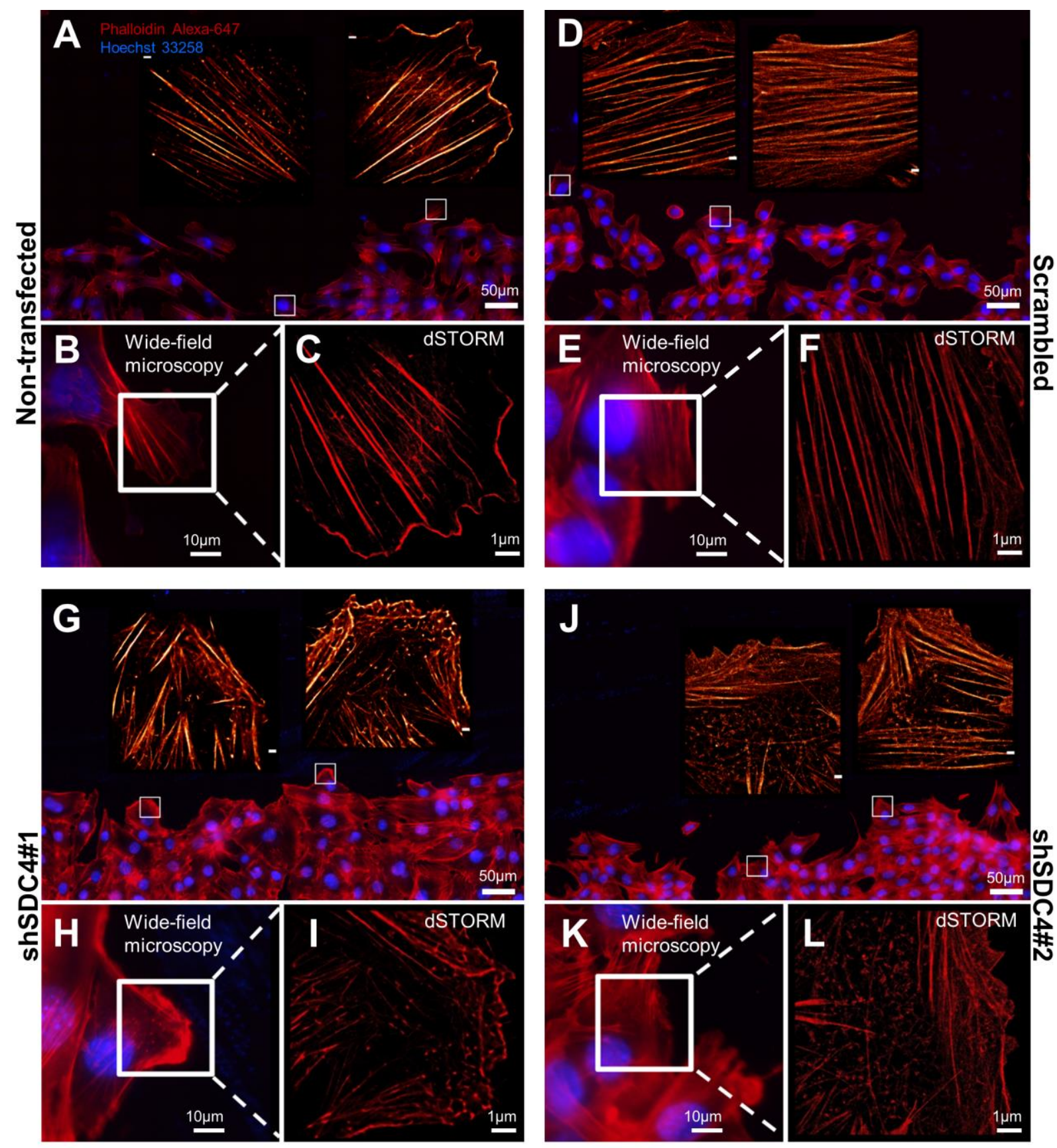

Figure 12. Direct stochastic optical reconstruction microscopy (dSTORM) analysis of the actin cytoskeleton after SDC4-silencing. Representative wide-field fluorescence and super-resolution dSTORM images show the actin skeletons of the cells adjacent to the cell-free zone in cultures of nontransfected $(A-C)$, scrambled $(D-F)$, shSDC4\#1 $(G-I)$ and shSDC4\#2 $(J-L)$ cell lines. Confluent monolayers were scratched, the cells were fixed $2 \mathrm{~h}$ later and actin filaments were stained with Alexa Fluor 647-conjugated phalloidin (red). Wide-field fluorescence images were obtained around the cellfree zone $(A, D, G, J$; higher magnification: $B, E, H, K)$. The insets of the wide-field fluorescence images depict ASTORM images of the lamellipodial regions of migrating cells adjacent to the cell-free zone $(A, B, D, E, G, H, J, K)$. Representative dSTORM images of lamellipodial actin structures are embedded in the original low-magnification images $(A, D, G, J$; bar: $1 \mu \mathrm{m})$ or are shown in separate higher magnification panels $(C, F, I, L)$. Nuclei are stained by Hoechst 33258 (blue). 


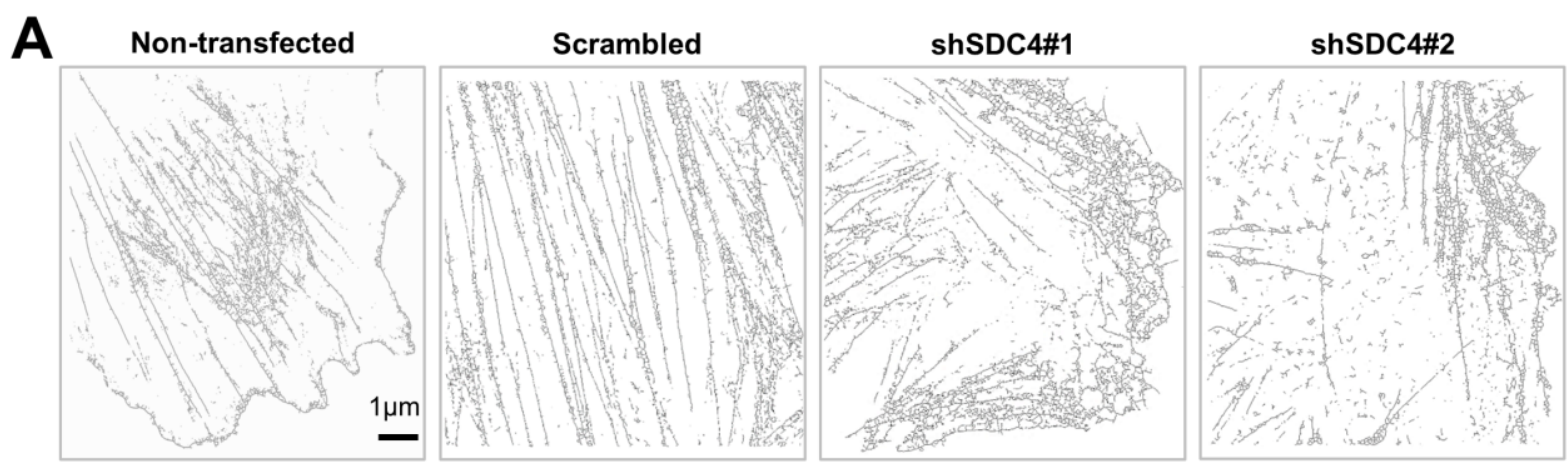

B

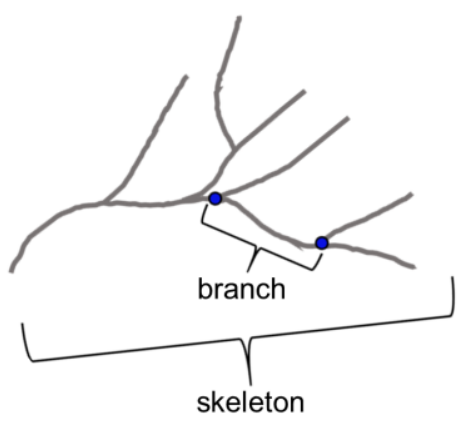

D

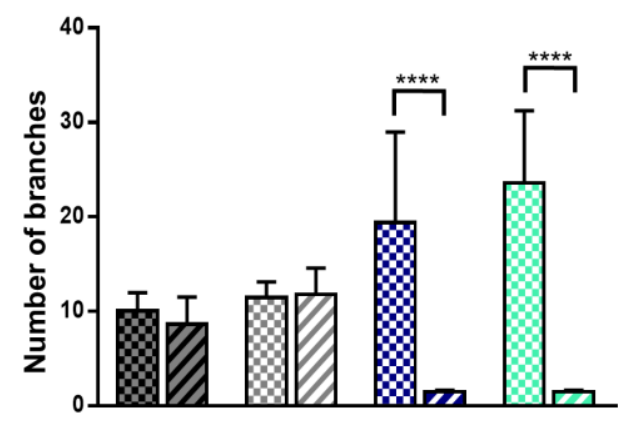

C

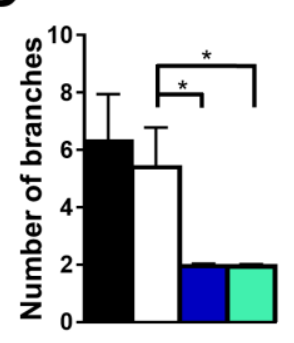

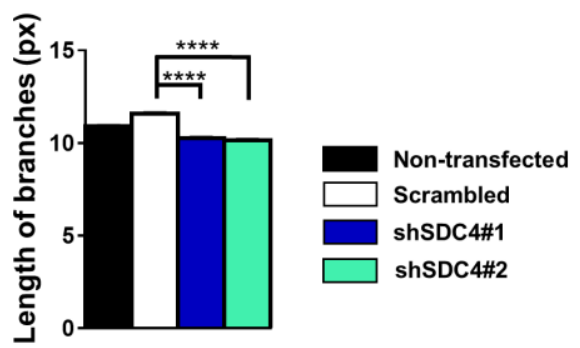

Figure 13. Skeletal analysis of dSTORM images of the lamellipodial actin network. The phalloidinstained lamellipodial actin cytoskeletons of the different cell lines were analyzed. Representative binary images converted from dSTORM images of the actin cytoskeleton are shown (A). Within the actin network, branching points divide the skeletons smaller branches $(B)$. The number of branches in the skeletons and the lengths of individual branches in the lamellipodial actin network in the whole binary images were quantified $(C)$. To measure the differences between the external and the internal region of the lamellipodial actin network, the average number and length of branches were compared in randomly selected areas (three areas in both external and internal regions, each $126 \times 124$ pixels in size) of the binary images $(D)$. Binary images of 6 cells/cell line were studied. Numbers of analyzed skeletons: 5,560 8,450/cell line; numbers of analyzed branches: 26,723-32,813/cell line. Number of analyzed skeletons in a single $126 \times 124$ pixels area: $2-69$, number of analyzed branches in a single $126 \times 124$ pixels area: 32-336. Data are shown as means + standard errors of the means; $* p<0.05, * * p<0.01, * * * p<0.001$ and $* * * * p<0.0001$.

The cells in the first row, next to the scratched areas were analyzed after actin filament labeling of the samples. For every cell line, panoramic maps of individual wide-field fluorescence images were generated (fig. 10-11.) to cover the whole area of the scratch wound (fig. 10-11.), and the lamellipodia of the migrating cells next to the wound were studied by dSTORM. Representative panoramic maps (in fig. 10-11.) and areas (fig. 12. A, D, $\mathrm{G}, \mathrm{J}$ ) of the panoramic maps are shown. 
SDC4 silencing altered the organization of the actin cytoskeleton by hindering the development of actin structures (fig. 12. G-L). The non-transfected and scrambled cells exhibited well-developed actin filaments (fig. 12. A-F), whereas this filamentous actin cytoskeletal structure was less pronounced and the lamellipodial actin network was less organized in SDC4 knockdown cells (fig. 12. G-L).

Next, dSTORM images of the actin cytoskeleton were converted to binary images (fig. 13. A) and analyzed further to quantify nanoscale changes in the actin network (fig. 13. B). The analysis of binary images of the lamellipodial actin filaments (fig. 13. A) revealed decreases in both the number of branches and the lengths of individual branches in the lamellipodial actin networks of SDC4 knockdown cells (fig. 13. C).

Both dSTORM and binary images suggested the presence of an actin-depleted inner region some micrometers away from the leading edge in SDC4 knockdown cell lines. Next, the nanoscale changes of the cortical (external) and the inner area of the lamellipodial actin network in the cells of cell lines were evaluated (fig. 13. D). Both the average number of branches (in each skeleton) and the lengths of individual branches were reduced in the inner region as compared to the external region of the lamellipodia in SDC4 knock-down cells. The results suggest that the lamellipodial actin structure in SDC4-silenced cells is inhomogeneous (i.e., actin-rich external region and actin-depleted inner area).

\subsection{Effect of SDC4 knockdown on focal adhesions}

Focal adhesions are dynamic structures that undergo cycles of assembly and disassembly; indeed, focal adhesion turnover is essential during cell migration. SDC4 plays a central role in the formation of focal adhesions organized around $\alpha 5 \beta 1$ integrin receptors [123].

To prove the migratory phenotype of the cells next to the cell-free zone, the focal adhesions were stained by anti-FAK antibody in all of the cell lines and FAK-stained focal adhesions were observed at the end of the stress fibers (fig. 14. A). Interestingly, both the size and the number of focal adhesions decreased in SDC4 knockdown cells (fig. 14. B, C). 

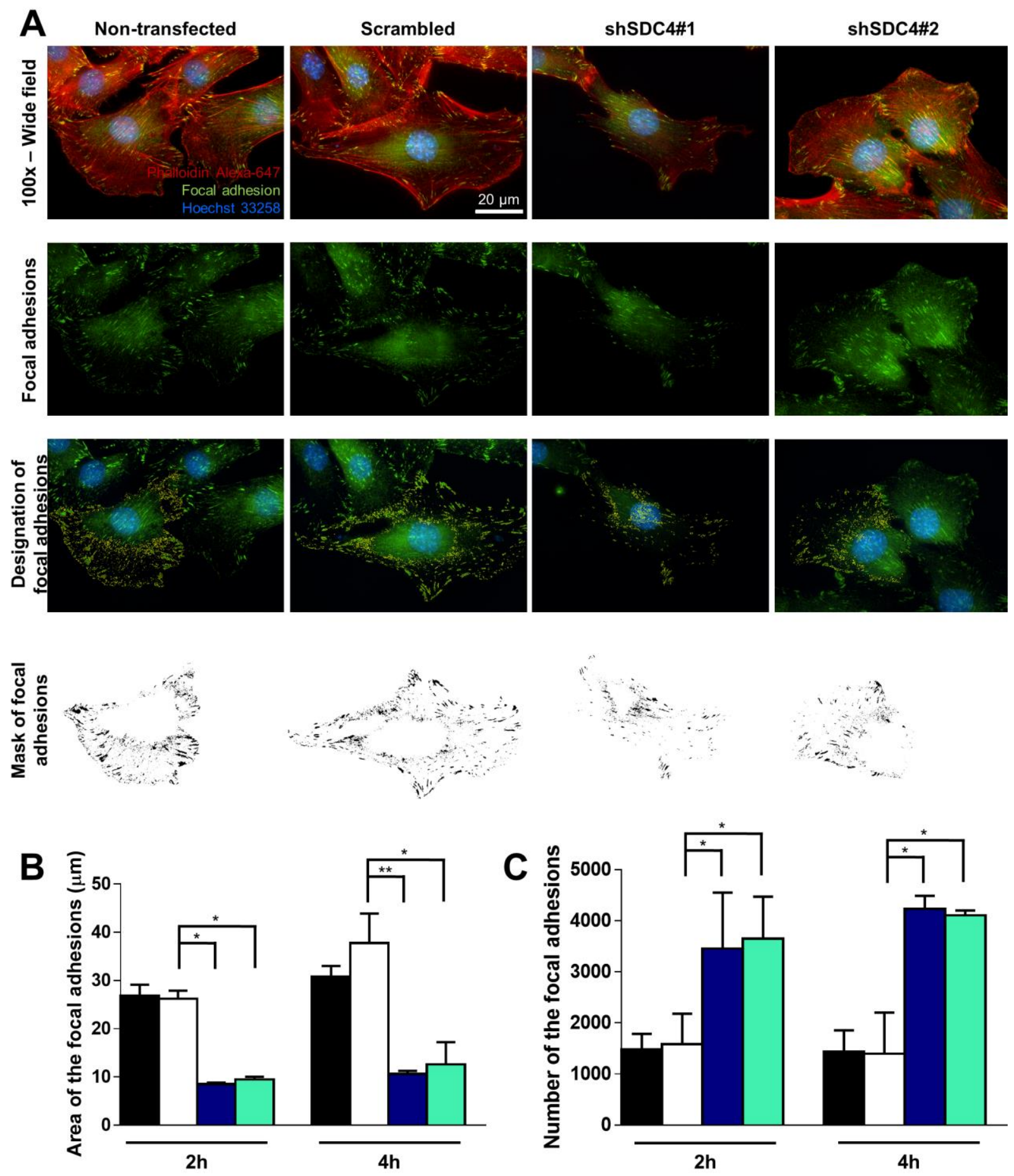

Figure 14. Distribution of focal adhesions in control and SDC4-silenced myoblasts. Representative $100 \times$ wide-field fluorescence images depict the actin cytoskeleton (Alexa Fluor 647-conjugated phalloidin: red) and focal adhesion distribution (anti-FAK antibody: green) of the migrating cells in different cell lines $2 \mathrm{~h}$ after wounding. Nuclei are stained by Hoechst 33258 (blue). To quantify focal adhesions, the focal adhesions were assigned in FAK stained fluorescence images. The area and the number of the focal adhesions were quantified 2 and $4 \mathrm{~h}$ after wounding the monolayer (B, C). Data are reported as means + standard errors of the means, $n=10$ cells $/$ cell line, 4 independent experiments were analyzed; *: $p<0.05 ; * *: p<0.01$. 


\subsection{SDC4 affects centrosome positioning and cell polarity}

For efficient cell migration the proper polarization of the cell [124], adequate positioning of the cellular compartments [125] and dynamic reconstruction of the actin cytoskeleton [126128] are required. As SDC4 silencing was shown to reduce myoblast migration, we next studied the polarization of SDC4 knockdown cells using centrosome localization, an indicator of cell polarity in migrating cells [107,129]. Specifically, the exact positions of the centrosomes were observed on immunostained samples obtained 2, 4 and $6 \mathrm{~h}$ after a wound scratch assay (Table 1., fig. 15.). Fluorescence images were captured after centrosome (anti- $\gamma$ tubulin) staining and used to generate panoramic maps of the entire scratched area.
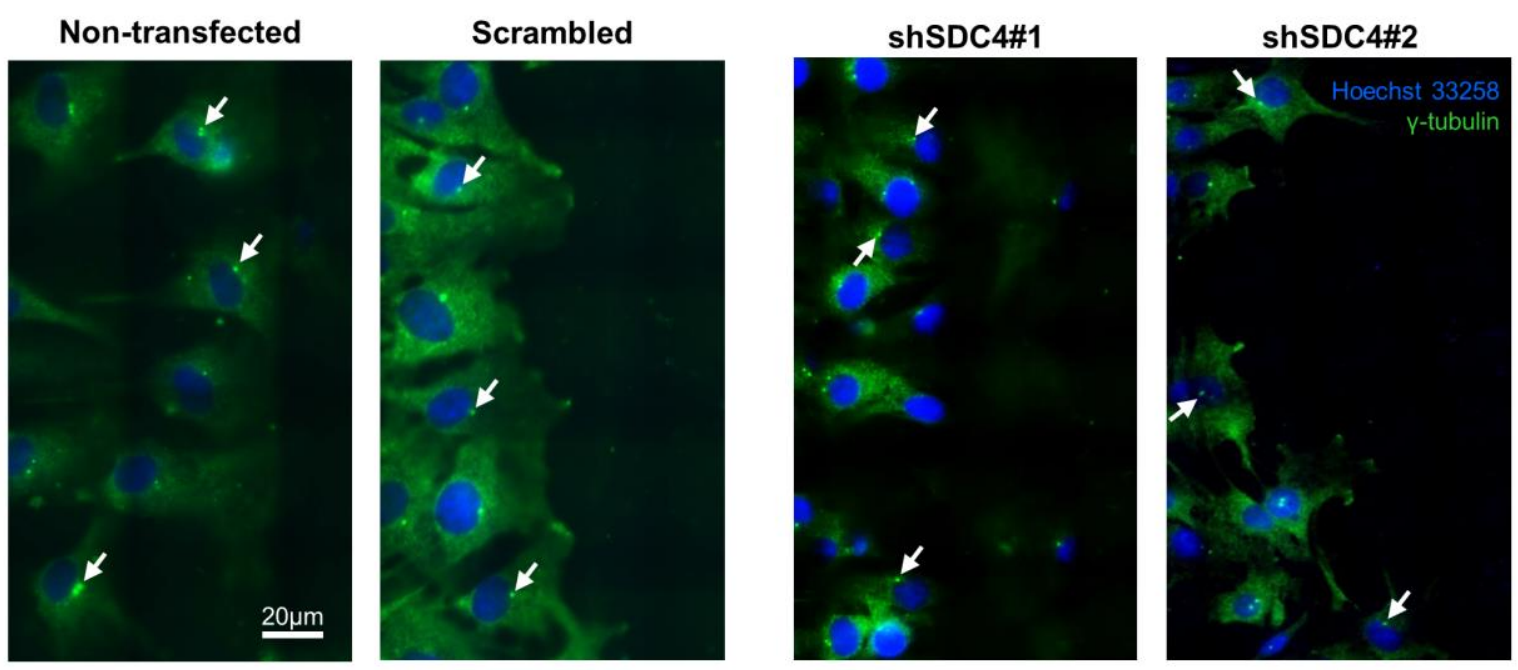

Figure 15. Representative wide-field fluorescence images of the studied cell lines depict the positions of centrosomes $\mathbf{2} \mathbf{h}$ after scratching. Anti- $\gamma$-tubulin-labeled centrosomes and Hoechst 33258stained nuclei are shown in green and blue, respectively. Arrows indicate the centrosomes.

To observe the polarization of the cells, we initially monitored the cells in the first row of the confluent monolayer located at the border of the cell-free zone (fig. 15). Two $\mathrm{h}$ after wounding, cells adjacent to the cell-free area were investigated using the nuclei as the points of reference (i.e., origins). The areas around the nuclei were divided into $30^{\circ}$ sectors, and centrosomes located in the $30^{\circ}$ circular sector facing toward the cell-free area were considered properly oriented (fig. 16. A). Figure 16. B depicts the numbers of centrosomes in the various sectors from experiments involving the different cell lines. Notably, SDC4 knockdown was associated with significantly fewer centrosomes in the $30^{\circ}$ circular sector facing toward the cell-free zone, indicating an improper reorientation of the centrosomes in these cells (fig. 16. B, C). In contrast, nearly all centrosomes of the scrambled and non-transfected cells were localized to this $30^{\circ}$ circular sector facing toward the cell-free area, indicating precise and 
proper regulation of centrosome positioning in these controls (fig. 16. B, C). There was no significant difference between the non-transfected and scrambled cells (fig. 16. C).

A

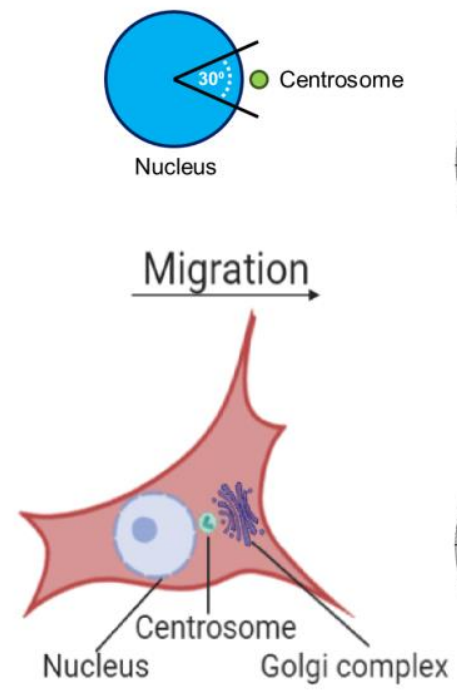

B

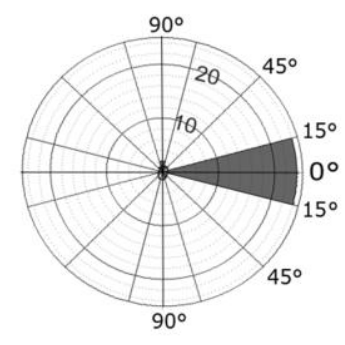

shSDC4\#1

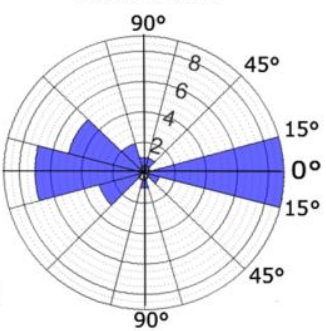

Scrambled

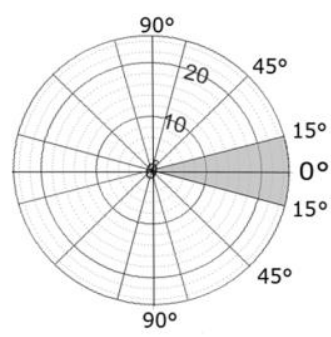

shSDC4\#2

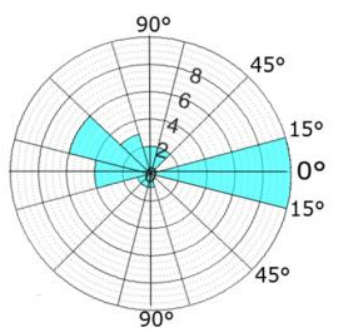

C
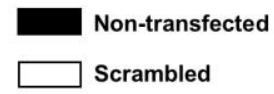

shSDC4\#1

$\square$ shSDC4\#2

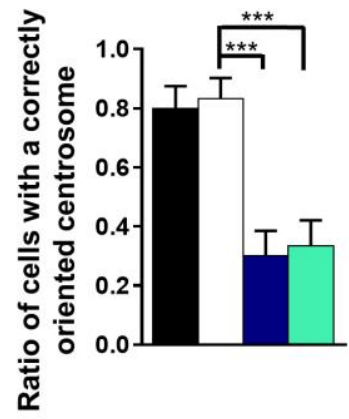

Figure 16. SDC4 affects centrosome positioning during migration. (A) Schematic representation of a polarized migratory cell. To quantify the positions of centrosomes, the nucleus was set as the origin, and centrosomes located in the $30^{\circ}$ circular sector facing toward the direction of wound closure were considered properly located. (B) Pie charts (i.e., polar histograms) show the localization of centrosomes in different cell lines. The plane was partitioned into $30^{\circ}$ circular sectors with the nucleus as the origin. The radius of each circular sector represents the number of cells with centrosomes located in that $30^{\circ}$ sector. $N=3$ independent experiments. Thirty cells were analyzed per cell line. $(C)$ Quantification of the results shown in $(B)$. The graph presents the ratios of centrosomes in the $30^{\circ}$ sector facing the cell-free area. Data are shown as means + standard errors of the means; $* * * p<0.001$.

To analyze the time dependency of centrosome reorientation, the position of centrosomes was studied 2, 4, and $6 \mathrm{~h}$ after wounding (Table 1). For this large number of items, a new nomenclature was introduced based on a previous work of Gotlieb et al. [130]. Considering the nucleus as an origin, the position of the centrosomes was classified into "toward", "middle", "away" positions based on the position of the centrosomes relative to the wound, i.e., from the direction of the wound to the nucleus "toward" in the nucleus line, i.e. "middle" or on the opposite side, i.e. "away". 


\begin{tabular}{|c|c|c|c|c|c|c|c|c|c|c|c|c|}
\hline & \multicolumn{12}{|c|}{ Time after scratch } \\
\hline & \multicolumn{4}{|c|}{$2 \mathrm{~h}$} & \multicolumn{4}{|c|}{$4 \mathrm{~h}$} & \multicolumn{4}{|c|}{$6 \mathrm{~h}$} \\
\hline & $\begin{array}{c}\text { Non- } \\
\text { transfected }\end{array}$ & Scrambled & shSDC4\#1 & shSDC4\#2 & $\begin{array}{c}\text { Non- } \\
\text { transfected }\end{array}$ & Scrambled & shSDC4\#1 & shSDC4\#2 & $\begin{array}{c}\text { Non- } \\
\text { transfected }\end{array}$ & Scrambled & shSDC4\#1 & shSDC4\#2 \\
\hline \multicolumn{13}{|c|}{ Toward } \\
\hline 1 st & $80 \pm 4.0$ & $83 \pm 1.0$ & $8 \pm 1.0$ & $7 \pm 1.5$ & $86 \pm 2.5$ & $88 \pm 1.0$ & $15 \pm 1.0$ & $14 \pm 2.0$ & $94 \pm 4.0$ & $92 \pm 3.0$ & $27 \pm 3.5$ & $25 \pm 3.5$ \\
\hline 2nd & $84 \pm 1.5$ & $87 \pm 2.5$ & $6 \pm 1.5$ & $4 \pm 0.5$ & $84 \pm 0.5$ & $89 \pm 5.0$ & $12 \pm 1.0$ & $16 \pm 2.0$ & $97 \pm 2.0$ & $98 \pm 1.5$ & $35 \pm 2.5$ & $27 \pm 2.5$ \\
\hline \multicolumn{13}{|c|}{ Middle } \\
\hline $1 \mathrm{st}$ & $8 \pm 1.0$ & $8 \pm 1.5$ & $22 \pm 2.0$ & $20 \pm 3.0$ & $2 \pm 4.5$ & $4 \pm 2.0$ & $17 \pm 2.5$ & $19 \pm 3.0$ & $2 \pm 2.0$ & $5 \pm 1.5$ & $16 \pm 1.5$ & $23 \pm 1.0$ \\
\hline 2nd & $6 \pm 0.5$ & $7 \pm 1.0$ & $18 \pm 3.0$ & $15 \pm 5.5$ & $7 \pm 6.5$ & $5 \pm 8.0$ & $21 \pm 1.0$ & $14 \pm 3.0$ & $2 \pm 2.5$ & $1 \pm 0.5$ & $17 \pm 2.5$ & $12 \pm 1.5$ \\
\hline \multicolumn{13}{|c|}{ Away } \\
\hline 1st & $12 \pm 3.0$ & $9 \pm 2.5$ & $70 \pm 3.0$ & $73 \pm 4.5$ & $12 \pm 2.0$ & $8 \pm 1.0$ & $71 \pm 1.5$ & $67 \pm 1.0$ & $4 \pm 2.0$ & $3 \pm 1.5$ & $57 \pm 2.0$ & $52 \pm 2.5$ \\
\hline 2nd & $10 \pm 1.0$ & $6 \pm 1.5$ & $76 \pm 1.5$ & $81 \pm 5.0$ & $9 \pm 6.0$ & $11 \pm 3.0$ & $67 \pm 2.0$ & $70 \pm 1.0$ & $1 \pm 0.5$ & $1 \pm 1.0$ & $52 \pm 5.0$ & $61 \pm 1.0$ \\
\hline
\end{tabular}

Table 1. Comparison of the effect of SDC4 silencing on centrosome reorientation in the 1st and 2nd row of myoblasts along the wound edge. The position of the centrosomes was classified as "toward", "middle" and "away", considering the nucleus as the origin and the cell-free zone as reference. Four independent experiments, $n=100-100$ cells/cell lines in each row. 
The number of centrosomes facing the wound edge increased in all cell lines during the 6 $\mathrm{h}$ period in both $1 \mathrm{st}$ and 2 nd row. Analysis of centrosome position along the wound edge revealed that in $83 \%$ of the scrambled cells in the first row the centrosomes were located toward the wound edge (between the nucleus and the wound edge) $2 \mathrm{~h}$ after wounding and $94 \%$ of the cells $6 \mathrm{~h}$ following wounding. In contrast, only 25-27\% of the SDC4-silenced cells presented centrosomes with a "toward" position $6 \mathrm{~h}$ after wounding. In scrambled cells, only a few numbers of cells exhibited "middle" (along the side of the nucleus), or "away" (between the nucleus of the monolayer behind the cells) localized centrosomes $6 \mathrm{~h}$ after scratching (Table 1). Based on these results, the reorientation of centrosomes during migration is delayed and underdeveloped in SDC4 knockdown cells.

\subsection{Polarized distribution of SDC4 during migration}

The former experiments demonstrated that SDC4 influences cellular polarity indicated by the impaired centrosome positioning and migration properties of myoblasts. Next, we examined the intracellular distribution of SDC4 in control (scrambled) and SDC4-silenced cell lines in wide-field fluorescence images. According to immunostaining experiments, the amount of SDC4, considering all fluorescence signal intensities, was significantly higher in control cells than in SDC4-silenced cell lines (fig. 17. A, B, D). SDC4 accumulates in the quadrant of the migrating cells facing the wounded area (fig. 17. A) which points the direction of migration (fig. 17. C). Comparing the amount of SDC4 accumulated in the quadrant facing the wounded area (fig. 17. C) to the total of SDC4 level of the cells did not depict significant difference between the cell lines (fig. 17. D). Based on these results, the distribution of SDC4 does not change as a result of silencing; only the total amount of SDC4 is lower in knockdown cells.

Since the wide-field images showed cytoplasmic SDC4 staining, next we performed double immunostaining experiments and confocal imaging. The representative confocal image (fig. 18. A) depicts the weak cell membrane localization of SDC4 in a migrating cell. Since earlier we showed the co-localization of SDC4 with the anti-GM130 Golgi marker and SDC4 is a member of focal adhesions, next we tested the co-distribution of SDC4 with GM130 and FAK (fig. 18. B, C). The observed localization of SDC4 in the focal adhesions and cis-Golgi (fig. 18. B, C) can explain the vacuolar and punctate signals of SDC4 staining.

It is already shown by our research group, that the phospho- (Ser179 in human and Ser183 in mouse) SDC4 accumulates in the cytoplasm during cytokinesis [131]. For this 
reason, it cannot be excluded that the SDC4 signal in migrating cells partially originates from the cytoplasmic phosphorylated form.

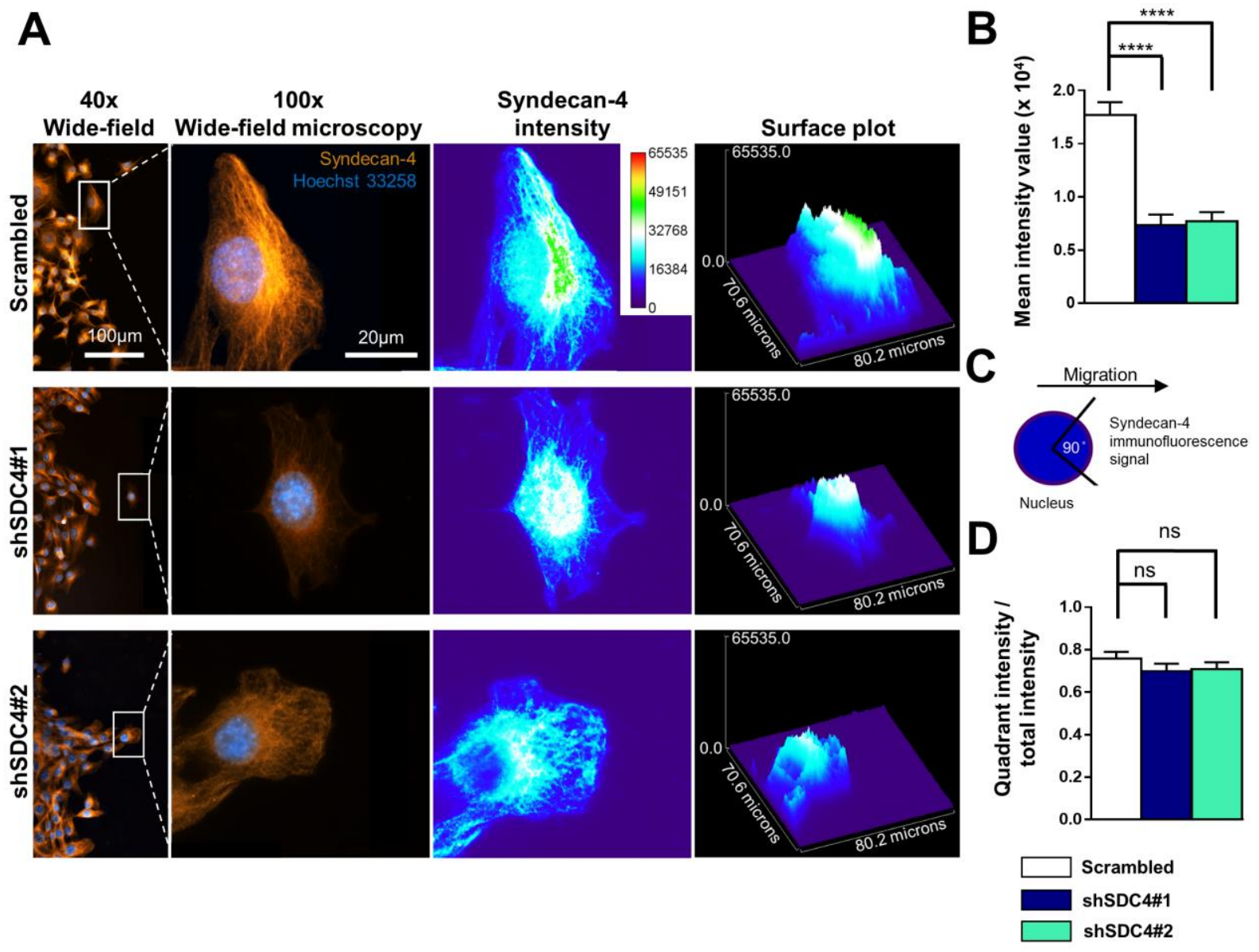

Figure 17. Distribution of SDC4 in migrating myoblasts. Representative images of the distribution of SDC4, marked orange, Alexa Fluor 568, and pseudo-color images about the intensity of the molecule. Nuclei are stained by Hoechst 33258 (blue) (A). The mean intensity value (B); the ratio of signal intensity of the quadrant pointing into the direction of migration (see schematic figure, C), and the total SDC4 intensity of the cells $(D)$ was calculated and compared in of the cell lines. Data are listed as means + standard errors of the means, $n=$ 30 cells / cell line were analyzed; ns: not significant; **** $p<0.0001$. 

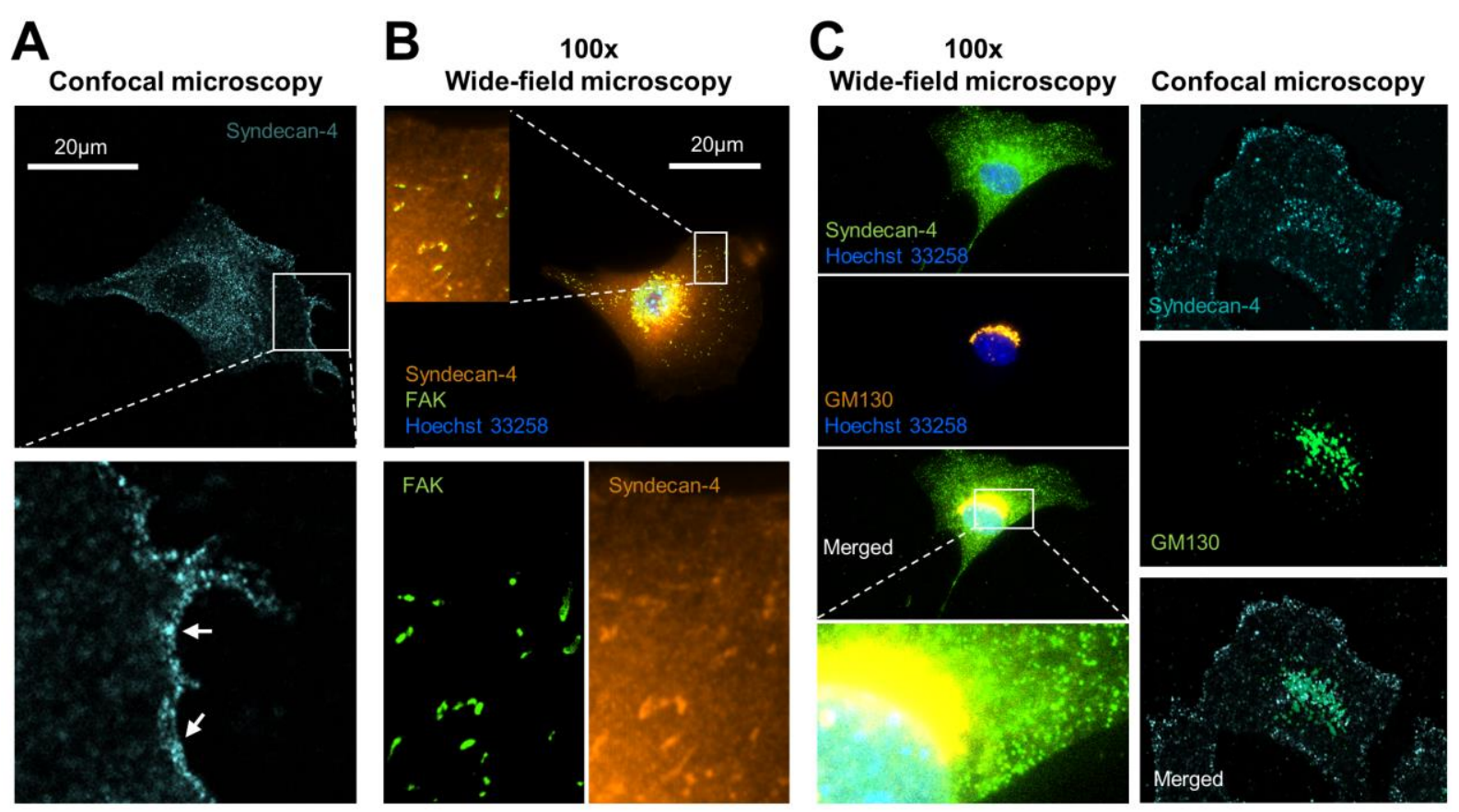

Figure 18. Distribution of SDC4, FAK, and GM130 in migrating myoblasts. Distribution of the cell membrane SDC4 of migrating myoblasts (white arrows) on representative confocal images (a cell of scrambled cell line) (A). SDC4 and FAK distribution in a migrating cell - wide field fluorescence image (scrambled cell) (B). Representative wide-field fluorescence and confocal image of GM130 (cis-Golgi marker) and SDC4 double staining in migrating scrambled cells $(C)$.

\section{6. $\quad$ SDC4 knockdown abrogates the intracellular $\mathrm{Ca}^{2+}$ gradient in migrating cells}

Normally, migrating cells exhibit a gradual increase in $\mathrm{Ca}^{2+}$ levels along the axis of migration. Accordingly, we next assessed the distribution of intracellular $\mathrm{Ca}^{2+}$ in SDC4silenced $\mathrm{C} 2 \mathrm{C} 12$ cells and compered to that seen in cells transfected with a scrambled target sequence. The front-rear $\mathrm{Ca}^{2+}$ distribution was studied in cells adjacent to the cell-free area in a scratch-wounded confluent culture (fig. 19. A). As expected, the intracellular $\mathrm{Ca}^{2+}$ concentration increased from the leading edge to the rear in control scrambled cells in (fig. 19. $\mathrm{B}, \mathrm{C}$ ). In contrast, this $\mathrm{Ca}^{2+}$ gradient was completely abolished in SDC4 knockdown cells (fig. 19. B, C). Since it has been reported that Fura Red tend to accumulate in the mitochondria [132], we explored whether the punctate structures can be observed in the $\mathrm{Ca}^{2+}$ indicatorloaded cell are mitochondria. Either control or SDC4-silenced cells exhibited distinct distribution for the $\mathrm{Ca}^{2+}$ indicators and the mitochondrial dye MitoTracker Deep Red, demonstrating that neither Fluo-4 nor Fura Red accumulated in the mitochondria in our experiments (data not shown). In summary, our findings demonstrate the essential role of SDC4 in cell polarity. 
A

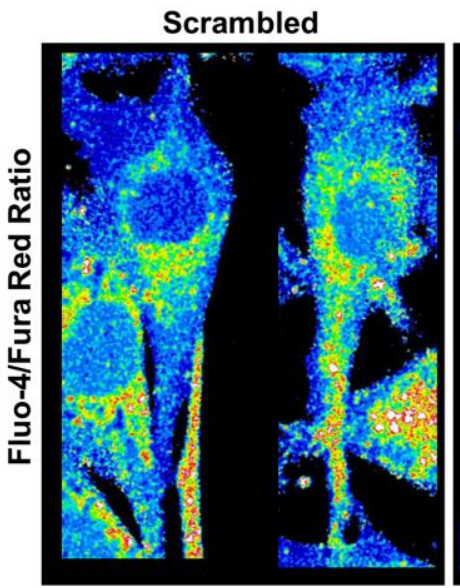

B

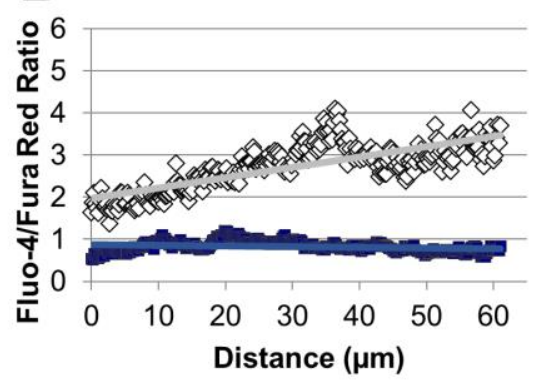

Scrambled trend
$\square$ Scrambled

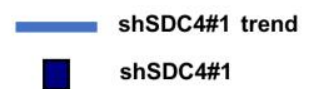

shSDC4\#1
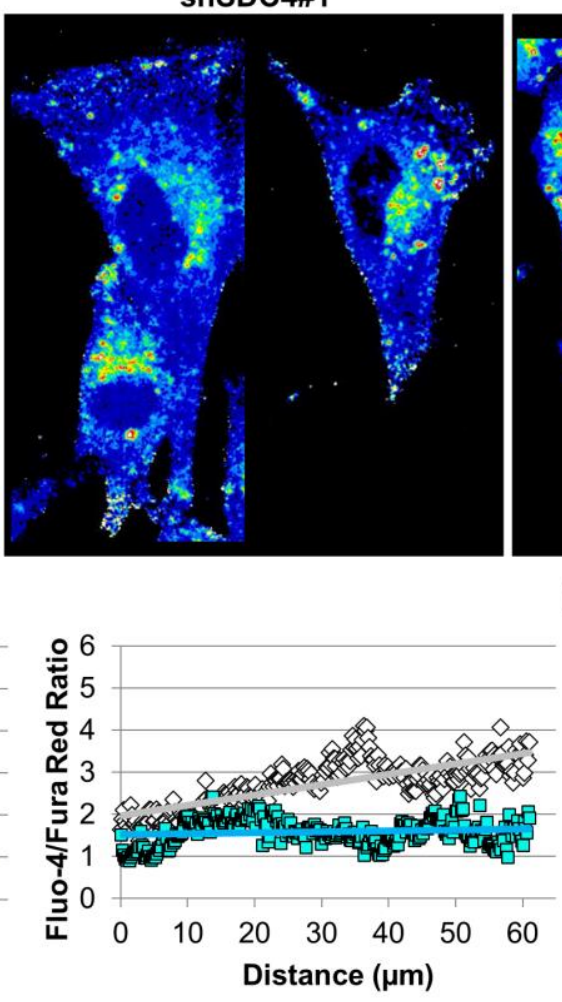

Distance $(\mu \mathrm{m})$
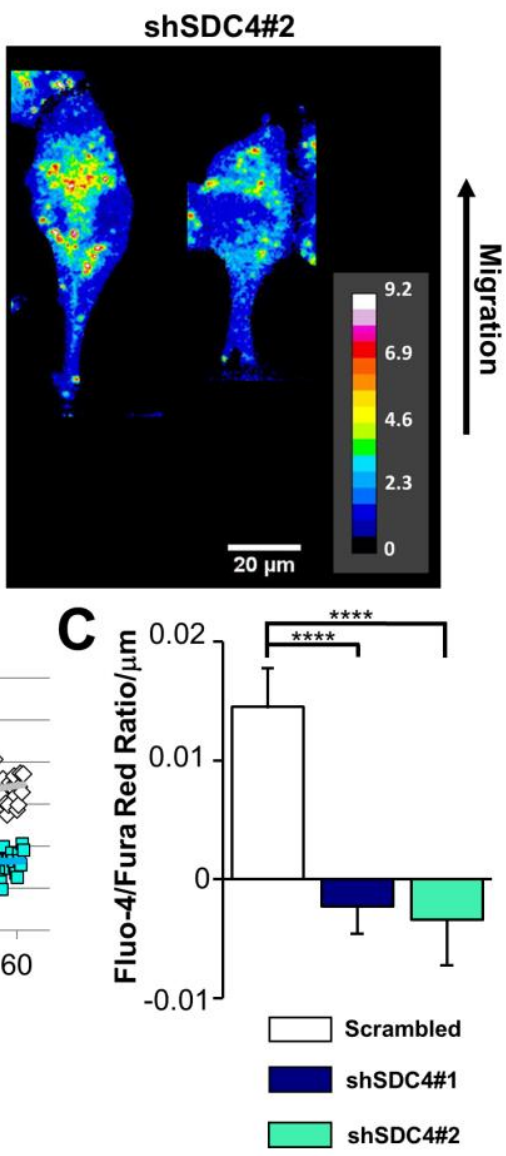

Figure 19. Distribution of intracellular $\mathbf{C a}^{2+}$ in migrating myoblasts. Effect of SDC4 silencing on the distribution of intracellular $\mathrm{Ca}^{2+}$ in migrating myoblasts (A). The ratio of Fluo-4 and Fura Red fluorescence, an indicator of the intracellular $\mathrm{Ca}^{2+}$ level, is shown in the representative pseudo-color images of scrambled, shSDC4\#1, and shSDC4\#2 cells (B). The ratio of Fluo-4 and Fura Red fluorescence was determined along the migration axis from the leading edge to the rear of cells following scratch wounding. The slopes of Fluo-4/Fura Red ratios along the migration axis in scrambled and SDC4 knockdown cells $(C)$. Migrating cells next to the cell-free zones $(n=8-12)$ revealed that SDC4 knockdown completely abolished $\mathrm{Ca}^{2+}$ gradient development in migrating cells. Data are shown as the means + standard errors of the means; $* * * * p<0.0001$.

\subsection{Inhibition of Rac1 does not restore the defective migratory phenotype of SDC4 knockdown cells}

Based on previous studies, SDC4 knockout causes an intracellular elevation of activated Rac1-GTPase [35,36,93,133-135]. The aim of our next experiment was to study how Rac1 inhibition affects cell migration, and whether it can improve the decreased migration of SDC4-silenced cells. The activity of Rac1 was specifically inhibited by NSC23766 treatment [136]. Myoblast migration was monitored for over $18 \mathrm{~h}$ by a random migration assay. During this analysis, the specific inhibition of Rac1 GTPase did not ameliorate the migration defect due to SDC4 knockdown (fig. 20. A). Interestingly, Rac1 inhibition caused further significant reduction in all examined parameters, including the total path of the cells, vectorial and maximum displacement, and average and maximum speed values in all cell lines (fig. 20. A). 
We transposed the migratory tracks (total paths) of the individual cells to a common origin to generate the static wind rose plots. The representative wind rose plots depicting the migratory tracks of the individual cells show the decreased motility of all cell lines upon treatment with the Rac1 inhibitor NSC23766 (fig. 21.). The representative plots clearly show the result of the combined effect of SDC4 silencing and Rac1 inhibition. Notably, the migratory parameters of SDC4-silenced cell lines further decreased following NSC23766 treatment.

The effectiveness of cell migration depends on two essential features: cell-speed and directional persistence. At the cellular level, directional persistence depends on the tenacity of lamellipodial protrusions and the stability of the trailing edge [35,135]. To ascertain the stability of the orientation of the cell migration, we also calculated the persistence index [136] in the case of the control and the SDC4 knockdown cell lines. The results show that silencing of SDC4 significantly decreases the persistence index of myoblasts measured after $18 \mathrm{~h}$ movement, and there was no significant difference between the non-transfected and scrambled cells (fig. 20. B). NSC23766 treatment of the cells further reduced persistence index in both SDC4 knockdown cell lines (fig. 20. B). The values of the persistence index over the timescale represent the time-dependency of the directional persistence showing that these differences can be constantly observed by comparing the cell lines (fig. 20. C). Interestingly, the persistence index of the untreated SDC4 knockdown cells was similar to the NSC23766-treated control lines; suggesting that neither high nor low activity of Rac1 favors directional persistence of the migration.

\section{8. $\quad$ SDC4 affects Tiam1 expression and localization}

Tiam1 has been identified as a GEF, acting as a specific activator of Rac1. Because specific inhibition of Rac1 reduced migration parameters for all cell lines, we explored whether we could identify alteration in either distribution or expression of Tiam1.

An asymmetrical Tiam1 distribution with an increased intensity towards the leading edge in the non-transfected and scrambled cell lines was identified whereas this peak in the Tiam1 intensity was absent in the SDC4-silenced cells (fig. 22. A). Heatmaps have been prepared to quantify the changes in Tiam1 signal intensity. The representative $2 \mathrm{D}$ and $3 \mathrm{D}$ pseudocolor images provide better visualization of this altered Tiam1 distribution: the high-intensity red area depicted an asymmetric Tiam1 enrichment that is mainly adjacent to the nucleus, and homogenous cytoplasmic blue-green intensity was observed in both SDC4-silenced cell lines. These quantified results confirm the pervious observations, as the mean intensity values in the individual cells are significantly decreased following SDC4 knockdown (fig. 22. B), 
indicative of reduced Tiam1 expression in these cells. To measure the variation in the pixel intensities, the standard deviation of Tiam1 intensity values within each cell were quantified. The standard deviation of intensity values in both SDC4-silenced cell lines were significantly lower. The pixel intensity values were closer to the mean intensity, whereas they are spread out over a wider range in the control cells (fig. 22. B, C). 

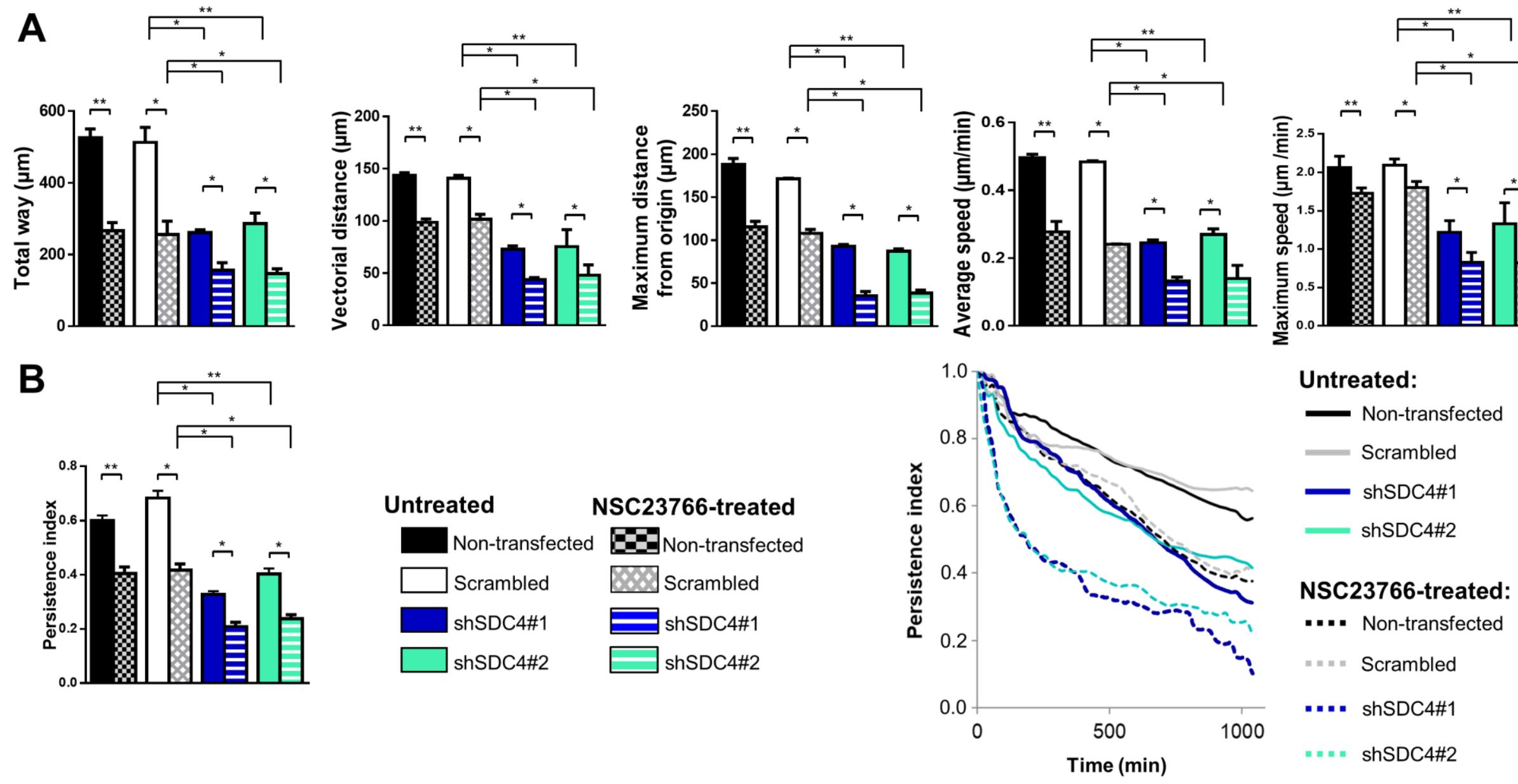

0.0

Figure 20. Effect of Rac1 inhibition on migration and persistence of control and SDC4 knockdown myoblasts in random migration assay. The total length of movement, the vectorial distance (real displacement of the cells), the maximum distance from the starting point, the average cell speed and the maximum speed of the cells are depicted in control and SDC4-silenced (shSDC4\#1, shSDC4\#2) C2C12 myoblasts either without or with NSC23766 (Rac1 inhibitor, 50 uM) treatment (A). Persistence index (vectorial distance/total path ratio) after $18 \mathrm{~h}$ and average persistence index over elapsed time $(B)$ were calculated. The total duration of live cell microscopy: $18 \mathrm{~h}$, frame rate:3/1 $\mathrm{h} ; n=4$ independent experiments; 62-114 cells/cell line; and 6-8 fields of view/experiment. Data are reported means of the independent experiments + SEM; $* * * * p<0.0001 ; * * * p<0.001 ; * * p<0.01 ; * p<0.05$. 

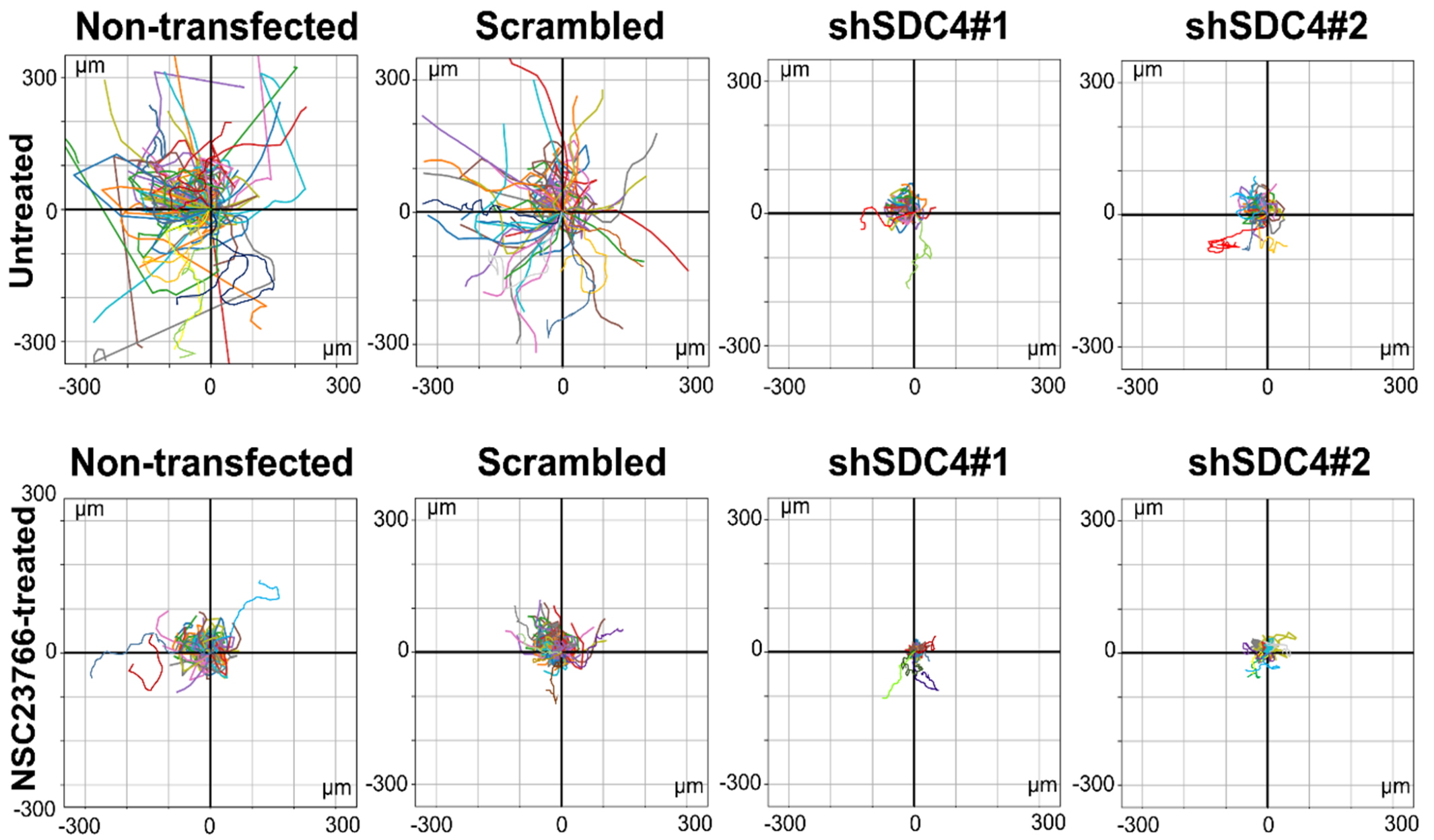

Figure 21. Representative wind-rose plots represent the total path of the cells. The so-called trajectories i.e., the the path of movement was shifted to a common origin. Each colored line demonstrates the total path of a single myoblast in the different cell lines. Total duration of live cell microscopy: $18 \mathrm{~h}$. 
A
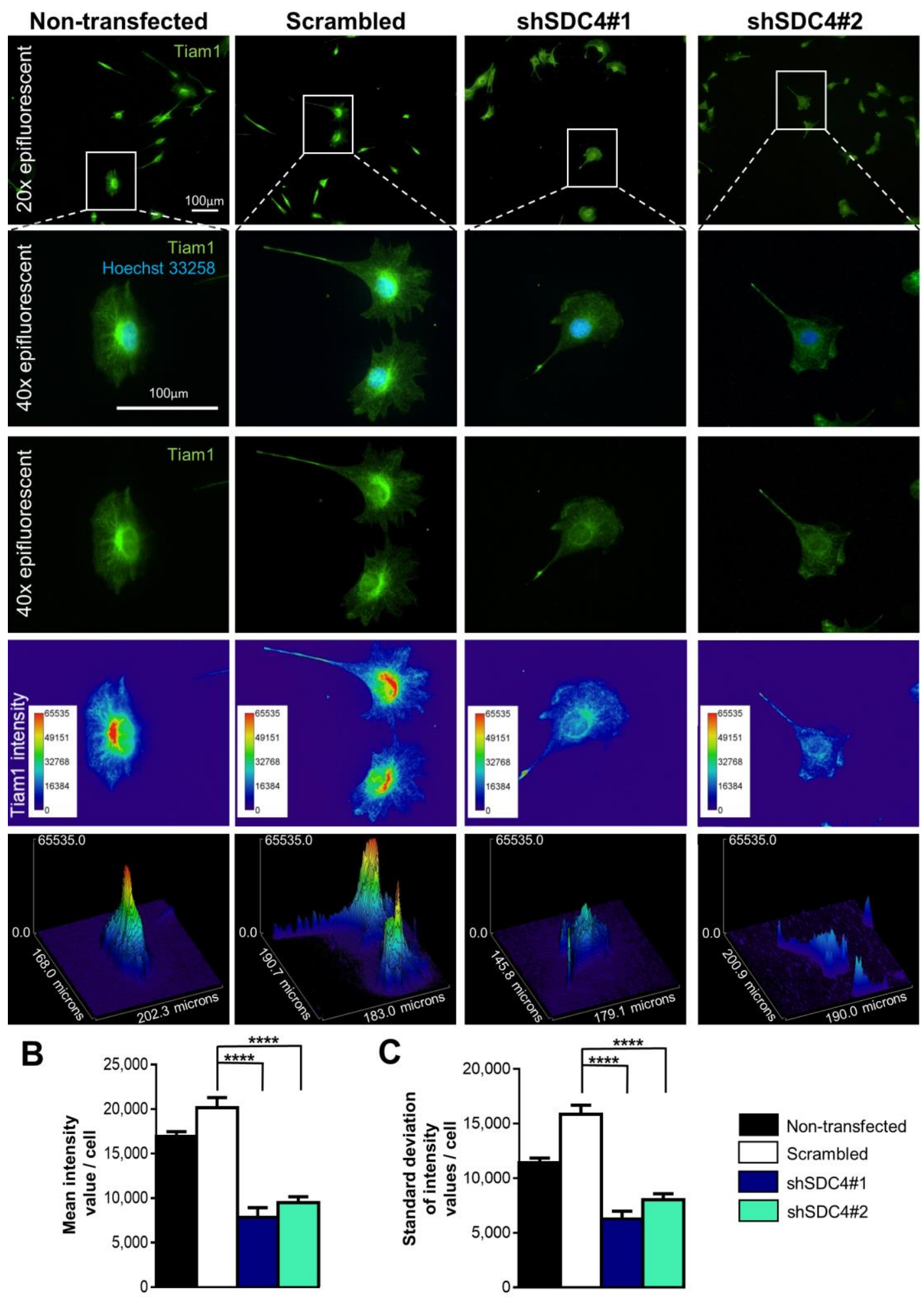

Figure 22. Tiam1 expression and distribution of the different cell lines. Representative images show Tiaml (green) distribution (A). The non-transfected and scrambled cells exhibit an asymmetric, polarized Tiaml arrangement, which is absent due to SDC4 silencing. Nuclei are blue (Hoechst 33258). Representative pseudocolor images $(2 D$ and $3 D)$ depict Tiam1 signal intensity. The color was assigned to each pixel based on the pixel intensity value according to the calibration bar (shown on left-hand sides of the images). The mean intensity values in the individual cells are quantified in $(B)$, and the average standard deviation values of the Tiaml intensities are depicted in $(C) . n=4-20$ cells/cell line were analyzed; data are reported as mean+ SEM; **** $p<0.0001$. 


\section{DISCUSSION}

Cell migration is an essential component of several physiological and pathological processes, including tissue regeneration. After injury, skeletal muscle tissue has a high degree of regenerative ability. This feature is also involved in pathological processes such as various muscle diseases (muscular dystrophies). Skeletal muscle regeneration consists of 4 phases, namely degeneration phase-1, inflammatory phase-2, regeneration phase- 3 and remodeling phase-4 [137]. In the first phase, muscle fiber damage occurs, in the second phase, neutrophil granulocytes and macrophages are activated, producing cytokines essential for the activation of satellite cells. In the third phase, the satellite cells activate, finally in the last phase, the process of extracellular reconstruction occurs, the restoration of the contractile apparatus and the process of angiogenesis are observed [137,138]. During regeneration, the activated satellite cells (i.e., myoblasts, activated skeletal muscle stem cells) proliferate, differentiate, migrate and fuse to form tubular, multi-nuclear myotubes. Myoblasts must be able to migrate in order to facilitate the cell-cell interactions and myoblast fusions required for muscle fiber formation during muscle regeneration and development.

SDCs, a family of transmembrane proteoglycans, have been reported to play crucial roles in tissue regeneration [139] and as a type I transmembrane proteoglycan, it is a component of focal adhesions. Besides its role as a structural molecule, it is involved in various signaling pathways. Our research team already demonstrated that SDC4 could influence myoblast proliferation, as SDC4 silencing reduced cell cycle progression from the G1 to the S phase and reduced the formation of mature myostatin, a negative regulator of muscle growth [140]. SDC4 knockout mice exhibited a decreased capacity for skin wound repair and angiogenesis [103], as well as inability to regenerate skeletal muscle following cardiotoxin-induced muscle necrosis [102]. In summary, SDC4 appears to play an essential role in skeletal muscle development and regeneration, although the exact mechanism underlying this phenomenon remains unclear [102].

The effect of SDC4 in cell migration has been previously studied over the years in different cell types such as hepatic satellite cells [141], lens epithelial cells [142], endothelial cells [35,122], human umbilical vein endothelial cells [143], human adult dermal fibroblasts [144], dendritic cells [145], neural crest cells [135], or fibroblasts [133]. This proteoglycan may contribute to the progression of tumor diseases by influencing the migration of tumor cells, such as lung adenocarcinoma [146] and hepatoma [147]; and also involved in the migration of dendritic cells in the context of allergic rhinitis [148] and B-cells in the context 
of arthritis [149]. A role of SDC4 has also been implicated in trophoblast migration and, consequently, the pathogenesis of preeclampsia [150]. More importantly, Shin et al. (2013) reported that SDC4 overexpression increased the migration of turkey satellite cells and increased the activation of RhoA GTPase, and these motile phenomena required the cytoplasmic domain of SDC4. Other studies observed reduced motility following SDC4 knockdown in different cell types, consistent with our current observations, whereas high SDC4 level promoted migration $[141,146,150]$. Based on the role of SDC4 in cellular proliferation and cell motility, SDC4 could be a prognostic marker in various tumor diseases [151] such as breast cancer [131,152], in esophageal cancer [153], in lung cancer [154,155], in papillary thyroid carcinoma [156], epithelial tumors [157] and also osteosarcoma [158].

Previous analyses of $\mathrm{C} 2 \mathrm{C} 12$ mouse myoblast cells revealed that SDC4 was the most prominent heparan sulfate proteoglycan in these cells when compared with SDC1, SDC2, SDC3, glypican, or perlecan, thus suggesting an important role for SDC4 in C2C12 myoblast cells. However, the observed upregulation of SDC1, SDC2 and SDC3 mRNAs after SDC4 silencing [140] suggests that other members of the SDC family may compensate at least partially for the loss of SDC4.

Given the significant role of SDC4 in cell migration and cytoskeletal organization, we hypothesized that this proteoglycan influences cell polarity, centrosome positioning, and intracellular $\mathrm{Ca}^{2+}$ distribution during cell migration.

In this thesis I demonstrated that SDC4 plays a crucial role in myoblast migration, due to its presence, we can speak of an efficient, dynamic and coordinated cell migration considering both intracellular processes and cell motility. The most striking change in the absence of SDC4 is that cells travel a shorter path, in addition, the persistence of cell migration deteriorates. During our observation, we found that in the absence of SDC4, myoblasts encountered orientation difficulty. Cells undergo an uncontrolled, uncoordinated migration, also the rate of it is much slower than in the presence of SDC4 (fig. 23. A, B).

In our research, we examined myoblast migration with directional and random migration assays to obtain an even more complete picture of the processes. During the directional wound scratch assay, we wanted to mimic in vitro the process of wound healing. It can be clearly seen that the path taken by the cells and the velocity of these cells are strongly reduced by SDC4 knock-down (fig. 23. B). However, during directional migration we were only able to study cell motility for $8 \mathrm{~h}$. In the case of control cell lines, $8 \mathrm{~h}$ after insert removal, the cellfree zone was closed. We extended our experiments to an $18 \mathrm{~h}$ assay, providing an even more nuanced picture of cell migration in the presence and absence of SDC4. In order to determine 
the orientation and endurance of cells, we introduced the concept of persistence index. It became clear that the persistence of myoblasts is strongly reduced in the absence of SDC4, which can be attributed to the upset of cell polarization.

The aim of this thesis is to answer why this anomaly occurs and for which background processes the presence of SDC4 in migrating cells is essential.

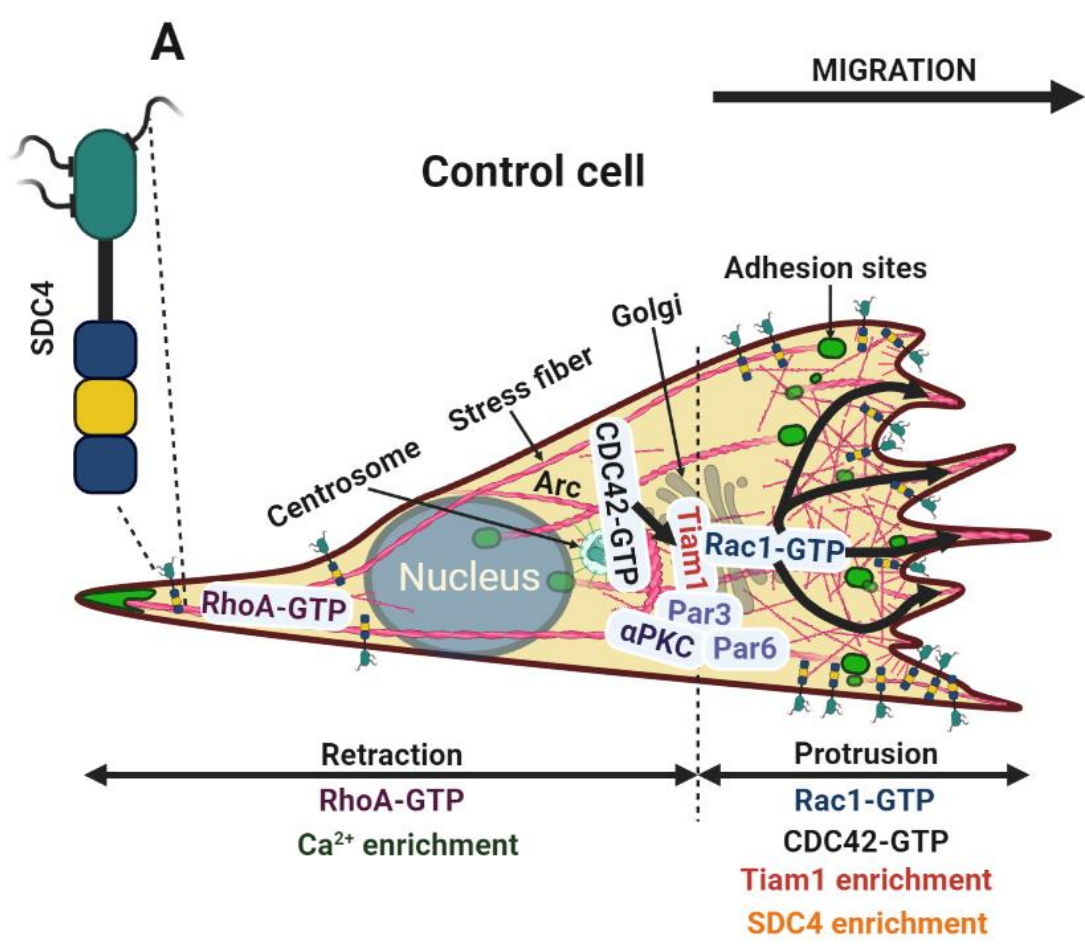

B

SDC4 knockdown cell

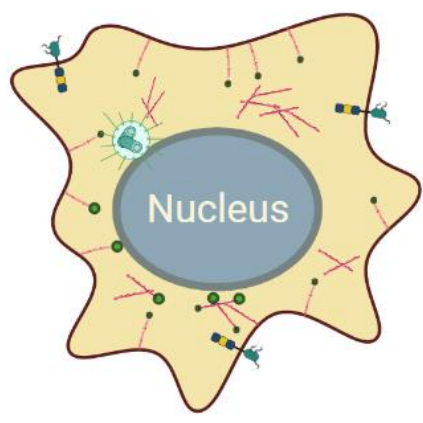

SDC4 absence delocalized Rac1-GTP

Tiam1 decrease

Figure 23. Schematic representation of the effect of SDC4 knockdown on cell polarity and migration with a characteristic shape. The intracellular distribution of $\mathrm{Ca}^{2+}$ and SDC4 in non-transfected control $(A)$ and SDC4 knockdown (B) myoblasts. In migrating cells, the formation of protruding leading edges is driven by Rac1/CDC42-GTPases favoring the generation of focal contacts, while the retraction and detachment occur at rear edges driven by RhoA-GTP. SDC4 distributes asymmetrically in migrating cells; and SDC4 knockdown resulted in the improper positioning of centrosomes, the absence of a frontrear $\mathrm{Ca}^{2+}$ gradient and disturbances in the nanoscale structures of the actin fibers. These abnormalities led to decreases in cell polarity and migration. The direction of migration is well defined, and the Tiaml is asymmetrically accumulated at the leading edge resulting in Racl activation and lamellipodia formation (A). Knocking-down of SDC4 resulted in decreased Tiam1 expression, abrogated the asymmetrical distribution of Tiam1, decreased the directional persistence of the movement and decreased cell motility. Par3, Par6: Partitioning defective 3,6; aPKC: atypical PKC (B). The figure was created with BioRender app.

Cell movement is highly dependent on the dynamic rearrangement of the actin cytoskeleton, a process closely related to the activation of members of the Rho family of small GTPases. Cdc42 and Rac play a critical role in the formation and organization of cortical actin networks and the protrusion of filopodia and lamellipodia [159]. The reorganization of cortical actin networks in the leading edge occurs as a result of increased activation of the Cdc42 / Rac pathway [160,161]. As cell movement progresses, focal 
adhesions reassemble behind the leading edge presumably reflecting the upregulation of Rho, thus providing attachment sites to anchor actin stress fibers and to support the contractile forces necessary for continued cell movement. The aim of this work was to investigate the nanoscale changes in the actin cytoskeleton upon SDC4 silencing in myoblasts. In our experience, SDC4 as a molecular conductor may play a role in the polymerization of actin at the leading edge of migrating myoblasts. In the absence of SDC4, the lamellipodial actin network is visibly altered, with actin stress fibers showing a less elongated, well-developed pattern.

Cell polarization, associated rearrangement of the actin cytoskeleton and cell-matrix relationships are key factors in cell migration. In addition to the integrins, SDC4 plays a cardinal role in the formation of focal adhesions. SDC4 binds directly to fibronectin, thereby promoting cell adhesion and cell migration, whereas the SDC4 / PKC $\alpha$ / RhoA signaling axis promotes focal adhesion formation [135,141]. Furthermore, downregulation of SDC4 suppressed integrin-mediated cell adhesion through inhibiting FAK phosphorylation [142], moreover the cytoplasmic domain of SDC4 interacts directly with $\alpha$-actinin [68], therefore the molecule is related to other also with adhesion components such as vinculin and zyxin [122] and the actin cytoskeleton [67]. Henceforward vinculin decoupling is induced from F-actin filaments in the case of SDC4 knockdown [122]. In a recent study on endothelial cells, SDC4 knockdown was shown to induce the decoupling of vinculin from F-actin filaments [122]. Interestingly, the interaction of PKC $\alpha$ and $\alpha$-actinin with SDC4 was shown to be reciprocal [162]. Moreover, SDC4 has been identified as a binding partner of dynamin II GTPase via its PH domain, and the resultant complex is a key regulator of focal adhesion and stress fiber formation in migrating cells [163]. Therefore, SDC4 serves as a central mediator in focal adhesion formation by bridging the interactions between integrins, fibronectin and intracellular molecules. Here we showed that both the number and size of FAK stained focal adhesions were decreased in SDC4 knockdown cells during migration. Consequently, the loss of SDC4 would affect cell motility via multiple mechanisms, including the observed changes in the lamellipodial actin cytoskeletal structure.

Intracellular $\mathrm{Ca}^{2+}$ levels are crucial for the physiological functioning of the cells such as migration. Both $\mathrm{Ca}^{2+}$ influx from the extracellular space via different plasma membrane $\mathrm{Ca}^{2+}$ channels and $\mathrm{Ca}^{2+}$ release from intracellular stores contribute to the cytosolic $\mathrm{Ca}^{2+}$ concentration. The level of intracellular $\mathrm{Ca}^{2+}$ influences the formation of the actin network, but it is also involved in the formation of various mechanical junctions and adhesions. The findings of this work suggest that SDC4 influences the development of this $\mathrm{Ca}^{2+}$ gradient, as 
demonstrated by its absence in SDC4 knockdown cells in association with decreased migration (fig. 23. A, B).

Intracellular levels of $\mathrm{Ca}^{2+}$ are characterized by a polarized distribution that increases within the migrating cell from the leading edge to the tail region. The presence of SDC4 influences this polarization, which is typical of the entire length of the cell. SDC4-influenced $\mathrm{Ca}^{2+}$ level has been previously described in several cell types such as podocytes [98], fibroblasts and keratinocytes [97], however a direct relationship between SDC4 and TRPC7 channels has still not been mapped [91]. Furthermore, the single knockdown of SDC4 in HaCaT keratinocytes did not affect the $\mathrm{Ca}^{2+}$ concentration, whereas the simultaneous silencing of both SDC1 and SDC4 decreased the cytosolic $\mathrm{Ca}^{2+}$ concentration in a TRPC4 channel-dependent manner [97]. $\mathrm{Ca}^{2+}$ gradient formation and FAK phosphorylation (Tyr397) are processes of outstanding importance for the reconstruction of focal adhesions [82,164]. The accumulation of phospho-FAK on the frontal side of the cell has been previously described $[53,165,166]$. Intracellular distribution and polarization of SDC4 affect local phospho-FAK levels and low levels of SDC4 in the rear of the cell may contribute to the disassembly of focal adhesions.

One of the indicators of cell polarization is the location of centrosomes within the cell $[107,129]$. The role of SDC4 in cytokinesis and the polarization of phospho-Ser179-SDC4 are known [131]. Crucial regulators of cellular polarity are cyclic, seesaw-like intracellular alternations of RhoA and Rac1 activity [36,96]. This study demonstrates that SDC4 influences the location of centrosomes within a cell. Since the orientation of the centrosome-nucleus axis depends on the balance of actin- and microtubule-mediated forces [167], changes in the cytoskeleton structure of actin may contribute to disorientation in the location of centrosomes. Furthermore, changes in the amount and level of Rac1 GTPase may also affect the intracellular misalignment of centrosomes in SDC4-silenced cells. This hypothesis is supported by the fact that Rac1 activity is localized to the leading edges of migrating SDC4sufficient cells, resulting in a persistent, constant migration [36].

The front-to-rear cell polarity required for migration depends on the activities of various members of the small GTPase Rho family. The rear of a migrating cell is defined by high levels of RhoA activity and subsequent actomyosin contractility, in addition to an increased $\mathrm{Ca}^{2+}$ concentration and the activation of $\mathrm{Ca}^{2+}$-dependent proteases required to cleave focal adhesion proteins. Interestingly, Tsai and colleagues suggested the presence of crosstalk between $\mathrm{Ca}^{2+}$ signaling and Rho GTPases that would coordinate the oscillations of these factors in the leading edges of migrating cells [45]. As noted, phospho-Ser179 SDC4 regulates 
both Rac1 GTPase activity [96] and intracellular $\mathrm{Ca}^{2+}$ level [97]. It would be interesting to determine whether these processes are coordinated simultaneously by SDC4 during cell migration.

Tiam1 has been identified as a nucleotide exchange factor (GEF). It has a fundamental role in vital physiological processes. Tiam1 is a specific activator of Rac1, Cdc42 and to a lesser extent, of RhoA [12,159]. The Par (comprising Par3, Par6 and atypical PKC)-Tiam1 complex stabilizes the front-rear polarization of migratory cells (fig. 23. A), thereby supporting persistent migration. The Par (comprising Par3, Par6, and atypical PKC)-Tiam1 complex stabilizes the front-rear polarization of migratory cells, thereby stimulating persistent and chemotactic migration. Meanwhile, in epithelial cells it controls the establishment of apical-basal polarity, thus showing the control of distinct forms of cellular polarity [37]. Tiam1-mediated Rac1 signaling is vital in establishing and maintaining of cell polarity, and it localizes the Rac1 activity at the leading edge of the migrating cells [23]. Impaired Tiam1 signaling was reported to inhibit the formation of front-rear polarization during migration thereby inhibiting persistent migration [37].

SDC4 is responsible for the localization of active Rac1 in membrane protrusions at the leading edge of cells by regulating $\mathrm{PKC} \alpha$ activity [36], which is necessary for persistent migration. Therefore, knocking-out of SDC4 increases the level of delocalized active Rac1 [36]. It is noteworthy that the direct relationship between the SDC4 coordinated persistent migration and the Tiam1-mediated Rac1 activation remains unknown. Earlier, our research group has reported that SDC4 binds and inhibits Tiam1, modulating the activity of Rac1 GTPase [140]. Thus, the SDC4-Tiam1 complex can regulate the local activity of Rac1.

Both the total amount of Rac1-GTP and its spatial distribution is critical for cell migration. Pankov et al. reported that both very highly activated Rac1 and very low Rac1 activity inhibit migration [168]. Knocking-out of SDC4 results in increased Rac1-GTP level [35,36,133-135], thus SDC4 is involved to maintain the low basal activity of Rac1. Here we report that SDC4 silencing decrease the migration of myoblasts. NSC23766 is a cellpermeable specific inhibitor of Rac1 activation by the Rac1-specific GEFs Tiam1 [136]. We hypothesized that Rac1 inhibitor treatment can improve the migratory parameters of SDC4 silenced cells, but the specific inhibition of Rac1 activity by NSC23766 did not rescue the effects of SDC4, rather exacerbated it. As NSC23766 treatment does not restore the migratory phenotype of the SDC4 knockdown cells, not only the active Rac1 level but the SDC4dependent localization of Rac1 activity is also important for the correct cell migration. 
For the effective cell migration, the establishment of a rear-front Rac1 gradient is essential. The SDC4 knockdown resulted in homogenous Tiam1 distribution, which might, in turn cause delocalized Rac1 activation (fig. 23. A, B). This can explain the reduced persistence and motility of SDC4 knockdown cells (fig. 23. A, B). In accordance with our observation, SDC4-null fibroblasts migrate randomly as a result of high delocalized Rac1 activity [36]. Interestingly, the polarized distribution of SDC4 was already shown during cytokinesis by its accumulation in the intercellular bridges during cell division [131]. Moreover, the directional persistence index of untreated SDC4 knockdown cells was similar to those of the NSC23766-treated non-transfected and scrambled lines; indicating that more than just the absolute amount of active Rac1 is important for the directionality of migration.

In this study, Tiam1 exhibited a strong asymmetrical localization toward the leading edge in non-transfected and scrambled cell lines. The perinuclear accumulation of Tiam1 can also be observed indicating that Tiam1 localizes not only in the leading-edge protrusions but also shows significant accumulation in the Golgi-network in migrating cells. As with other cellular constituents, such as centrosomes, the Golgi-apparatus requires a leader-oriented localization for effective cell migration. The level of Tiam1 expression seems to be correlated with cell motility [169]. In our study, we detected a significantly higher signal intensity of Tiam1 in non-transfected and scrambled cell lines, than in the SDC4 silenced cells (fig. 22. A). These findings may indicate increased expression of Tiam1 in control cell lines. SDC4 silencing reduces Tiam1 signal intensity and abrogates the strong, asymmetrical Tiam1 gradient towards the leading edge.

Given the role of Tiam1 and Rac1 in cell migration, several studies have explored the prognostic value of Tiam1 in patients with solid tumors. High Tiam1 expression has been significantly associated with shorter survival and positive lymphatic metastasis in patients with malignant solid tumors, and Tiam1 may present a promising prognostic biomarker and an effective therapeutic target for treating malignancies [170]. For example, a study has shown that Tiam1 expression correlated with cell motility in human breast cancer cells and is required to support the motile phenotype [169]. The authors reported the localization of endogenous Tiam1 to the Golgi, and demonstrated the role of this in Golgi reorientation, suggesting that it may support motility through a mechanism that is discrete from its known function in leading-edge dynamics [169]. Similarly, we identified accumulation of Tiam1 in intracellular compartments. In accordance with these Tiam1 expression data, the SDC4 knockdown cells in our model system exhibited both reduced expression of Tiam1 and reduced motility. 
Intercellular communication is essential for the survival of multicellular organisms through direct cell-cell contact as well as for transfer of secreted molecules or intercellular transfer of extracellular vesicles. Exosomes are extracellular vesicles 20-100 nm in diameter bounded by a phospholipid bilayer [171-173]. They are capable of horizontal transfer of many molecules such as actin, tubulin, DNA, RNA and other signaling proteins [174]. Due to their characteristic composition, they are suitable for diagnostics, while their specific absorption capacity may allow the delivery of drug molecules to the target cell. In addition, exosomes influence the process of cell migration in a paracrine and autocrine manner [175]. Leading cells secrete exosomes in order to help guide subsequent cells [176]. In addition, a mechanical role can be assumed, as a more efficient cell movement can be created by rolling on the exosomes [177]. The role of SDCs in exosome biogenesis is known and the role of exosomes in stabilizing adhesions has been described [178], however the signaling pathway has not been linked between them yet. One of our future aims is to map the molecular mechanisms associated with the SDC4 - exosome biogenesis and uptake - cell migration axis. Exosomes could be potential therapeutic targets that may help influence SDC4-coordinated signaling mechanisms in various lesions and diseases, as well as in tumor diseases.

Overall, we have identified new effects of SDC4 in the regulation of cell migration. Specifically, SDC4 silencing greatly reduces the migratory abilities of myoblasts. Presumably, this effect is due to a disturbance in cell polarization, which can be inferred from the shift in centrosome positioning relative to the nucleus and the absence of the intracellular $\mathrm{Ca}^{2+}$ gradient. Moreover, SDC4 affect Tiam1 expression and distribution, and directional persistence of migration. The reduced migration capability might also be attributed to changes in the nanoscale structure of the lamellipodial actin cytoskeleton and reductions in cell-matrix adhesions. These results indicate a critical role of SDC4 during myoblast migration, which may contribute to an exploration of the essential role of SDC4 in the development and regeneration of skeletal muscle. Our findings elucidate the multiple roles of SDC4 in myoblast cell migration, although these findings are likely applicable to other cell types, given the ubiquitous expression of SDC4. This increase in general knowledge about cell migration will likely facilitate the development of strategies for the further exploration of a wide range of physiological and pathological migratory processes. 


\section{FUNDING}

This research was supported by the National Research, Development and Innovation Office of Hungary [grant nos: GINOP-2.3.2-15-2016-00040 (MYOTeam), EFOP-3.6.2-162017-00006, NKFI FK 134684, NKFI K 128123 and NKFI K 132446]. The work was further supported by the János Bolyai Research Scholarship of the Hungarian Academy of Sciences, UNKP-19-4-SZTE-23 and UNKP-20-5-SZTE-162 New National Excellence Program of the Ministry for Innovation and Technology Sciences (to Anikó Keller-Pintér). The dSTORM measurements were funded by the Hungarian Brain Research Programme (grant no. 20171.2.1-NKP-2017-00002); the National Research, Development and Innovation Office of Hungary (grant nos. GINOP-2.3.2-15-2016-00036) and an EU-funded Hungarian Grant (grant no. EFOP-3.6.1-16-2016-00008). For live-cell microscopy imaging we acknowledge support from the LENDULET-BIOMAG Grant (grant no. 2018-342) and the European Regional Development Funds (grant nos. GINOP-2.3.2-15-2016-00006, GINOP-2.3.2-15-2016-00026, and GINOP-2.3.2-15-2016-00037). The research was also funded by EFOP 3.6.3-VEKOP-162017-00009 (to Dániel Becsky). 


\section{REFRENCES}

1. Mauro, A.; Adams, W.R. The structure of the sarcolemma of the frog skeletal muscle fiber. The Journal of biophysical and biochemical cytology 1961, 10(4)Suppl, 177-185, doi:10.1083/jcb.10.4.177.

2. Chang, N.C.; Rudnicki, M.A. Satellite cells: the architects of skeletal muscle. Current topics in developmental biology 2014, 107, 161-181, doi:10.1016/B978-0-12-4160224.00006-8.

3. Tedesco, F.S.; Dellavalle, A.; Diaz-Manera, J.; Messina, G.; Cossu, G. Repairing skeletal muscle: regenerative potential of skeletal muscle stem cells. The Journal of clinical investigation 2010, 120, 11-19, doi:10.1172/JCI40373.

4. $\quad$ Atkins, B.Z.; Lewis, C.W.; Kraus, W.E.; Hutcheson, K.A.; Glower, D.D.; Taylor, D.A. Intracardiac transplantation of skeletal myoblasts yields two populations of striated cells in situ. The Annals of thoracic surgery 1999, 67, 124-129, doi:10.1016/s0003-4975(98)01197-7.

5. Trepat, X.; Chen, Z.; Jacobson, K. Cell migration. Comprehensive Physiology 2012, 2, 2369-2392, doi:10.1002/cphy.c110012.

6. Ridley, A.J.; Schwartz, M.A.; Burridge, K.; Firtel, R.A.; Ginsberg, M.H.; Borisy, G.; Parsons, J.T.; Horwitz, A.R. Cell migration: integrating signals from front to back. Science 2003, 302, 1704-1709, doi:10.1126/science.1092053.

7. Hu, Y.L.; Li, S.; Miao, H.; Tsou, T.C.; del Pozo, M.A.; Chien, S. Roles of microtubule dynamics and small GTPase Rac in endothelial cell migration and lamellipodium formation under flow. Journal of vascular research 2002, 39, 465-476, doi:10.1159/000067202.

8. $\quad$ Burnette, D.T.; Manley, S.; Sengupta, P.; Sougrat, R.; Davidson, M.W.; Kachar, B.; Lippincott-Schwartz, J. A role for actin arcs in the leading-edge advance of migrating cells. Nature cell biology 2011, 13, 371-381, doi:10.1038/ncb2205.

9. Krause, M.; Gautreau, A. Steering cell migration: lamellipodium dynamics and the regulation of directional persistence. Nature reviews. Molecular cell biology 2014, 15, 577 590, doi:10.1038/nrm3861.

10. Vicente-Manzanares, M.; Webb, D.J.; Horwitz, A.R. Cell migration at a glance. Journal of cell science 2005, 118, 4917-4919, doi:10.1242/jcs.02662.

11. Mardakheh, F.K.; Self, A.; Marshall, C.J. RHO binding to FAM65A regulates Golgi reorientation during cell migration. Journal of cell science 2016, 129, 4466-4479, doi:10.1242/jcs.198614.

12. Small, J.V.; Herzog, M.; Anderson, K. Actin filament organization in the fish keratocyte lamellipodium. The Journal of cell biology 1995, 129, 1275-1286, doi:10.1083/jcb.129.5.1275.

13. Svitkina, T.M.; Verkhovsky, A.B.; McQuade, K.M.; Borisy, G.G. Analysis of the actinmyosin II system in fish epidermal keratocytes: mechanism of cell body translocation. The Journal of cell biology 1997, 139, 397-415, doi:10.1083/jcb.139.2.397.

14. Pollard, T.D.; Borisy, G.G. Cellular motility driven by assembly and disassembly of actin filaments. Cell 2003, 112, 453-465, doi:10.1016/s0092-8674(03)00120-x.

15. Huxley, A.F.; Niedergerke, R. Measurement of muscle striations in stretch and contraction. The Journal of physiology 1954, 124, 46-47P.

16. Huxley, A.F.; Niedergerke, R. Structural changes in muscle during contraction; interference microscopy of living muscle fibres. Nature 1954, 173, 971-973, doi:10.1038/173971a0.

17. Mullins, R.D.; Kelleher, J.F.; Xu, J.; Pollard, T.D. Arp2/3 complex from Acanthamoeba binds profilin and cross-links actin filaments. Molecular biology of the cell 1998, 9, 841-852, doi:10.1091/mbc.9.4.841.

18. Mullins, R.D.; Heuser, J.A.; Pollard, T.D. The interaction of Arp2/3 complex with 
actin: nucleation, high affinity pointed end capping, and formation of branching networks of filaments. Proceedings of the National Academy of Sciences of the United States of America 1998, 95, 6181-6186, doi:10.1073/pnas.95.11.6181.

19. Drees, F.; Pokutta, S.; Yamada, S.; Nelson, W.J.; Weis, W.I. Alpha-catenin is a molecular switch that binds E-cadherin-beta-catenin and regulates actin-filament assembly. Cell 2005, 123, 903-915, doi:10.1016/j.cell.2005.09.021.

20. Krause, M.; Dent, E.W.; Bear, J.E.; Loureiro, J.J.; Gertler, F.B. Ena/VASP proteins: regulators of the actin cytoskeleton and cell migration. Annual review of cell and developmental biology 2003, 19, 541-564, doi:10.1146/annurev.cellbio.19.050103.103356.

21. Hall, A. Rho family GTPases. Biochemical Society transactions 2012, 40, 1378-1382, doi:10.1042/BST20120103.

22. Hodge, R.G.; Ridley, A.J. Regulating Rho GTPases and their regulators. Nature reviews. Molecular cell biology 2016, 17, 496-510, doi:10.1038/nrm.2016.67.

23. Iden, S.; Collard, J.G. Crosstalk between small GTPases and polarity proteins in cell polarization. Nature reviews. Molecular cell biology 2008, 9, 846-859, doi:10.1038/nrm2521.

24. Bos, J.L.; Rehmann, H.; Wittinghofer, A. GEFs and GAPs: critical elements in the control of small G proteins. Cell 2007, 129, 865-877, doi:10.1016/j.cell.2007.05.018.

25. Nobes, C.D.; Hall, A. Rho, rac, and cdc42 GTPases regulate the assembly of multimolecular focal complexes associated with actin stress fibers, lamellipodia, and filopodia. Cell 1995, 81, 53-62, doi:10.1016/0092-8674(95)90370-4.

26. Itoh, R.E.; Kurokawa, K.; Ohba, Y.; Yoshizaki, H.; Mochizuki, N.; Matsuda, M. Activation of rac and cdc42 video imaged by fluorescent resonance energy transfer-based single-molecule probes in the membrane of living cells. Molecular and cellular biology 2002, 22, 6582-6591, doi:10.1128/mcb.22.18.6582-6591.2002.

27. Ridley, A.J. Rho GTPases and actin dynamics in membrane protrusions and vesicle trafficking. Trends in cell biology 2006, 16, 522-529, doi:10.1016/j.tcb.2006.08.006.

28. Mullins, R.D. How WASP-family proteins and the Arp2/3 complex convert intracellular signals into cytoskeletal structures. Current opinion in cell biology 2000, 12, 9196, doi:10.1016/s0955-0674(99)00061-7.

29. Machesky, L.M.; Gould, K.L. The Arp2/3 complex: a multifunctional actin organizer. Current opinion in cell biology 1999, 11, 117-121, doi:10.1016/s0955-0674(99)80014-3.

30. Machesky, L.M.; Mullins, R.D.; Higgs, H.N.; Kaiser, D.A.; Blanchoin, L.; May, R.C.; Hall, M.E.; Pollard, T.D. Scar, a WASp-related protein, activates nucleation of actin filaments by the Arp2/3 complex. Proceedings of the National Academy of Sciences of the United States of America 1999, 96, 3739-3744, doi:10.1073/pnas.96.7.3739.

31. Rohatgi, R.; Ma, L.; Miki, H.; Lopez, M.; Kirchhausen, T.; Takenawa, T.; Kirschner, M.W. The interaction between N-WASP and the Arp2/3 complex links Cdc42-dependent signals to actin assembly. Cell 1999, 97, 221-231, doi:10.1016/s0092-8674(00)80732-1.

32. Oleinik, N.V.; Helke, K.L.; Kistner-Griffin, E.; Krupenko, N.I.; Krupenko, S.A. Rho GTPases RhoA and Rac1 mediate effects of dietary folate on metastatic potential of A549 cancer cells through the control of cofilin phosphorylation. The Journal of biological chemistry 2014, 289, 26383-26394, doi:10.1074/jbc.M114.569657.

33. Mertens, A.E.; Pegtel, D.M.; Collard, J.G. Tiam1 takes PARt in cell polarity. Trends in cell biology 2006, 16, 308-316, doi:10.1016/j.tcb.2006.04.001.

34. Ten Klooster, J.P.; Evers, E.E.; Janssen, L.; Machesky, L.M.; Michiels, F.; Hordijk, P.; Collard, J.G. Interaction between Tiam1 and the Arp2/3 complex links activation of Rac to actin polymerization. The Biochemical journal 2006, 397, 39-45, doi:10.1042/BJ20051957.

35. Tkachenko, E.; Elfenbein, A.; Tirziu, D.; Simons, M. Syndecan-4 clustering induces cell migration in a PDZ-dependent manner. Circulation research 2006, 98, 1398-1404, doi:10.1161/01.RES.0000225283.71490.5a. 
36. Bass, M.D.; Roach, K.A.; Morgan, M.R.; Mostafavi-Pour, Z.; Schoen, T.; Muramatsu, T.; Mayer, U.; Ballestrem, C.; Spatz, J.P.; Humphries, M.J. Syndecan-4-dependent Rac1 regulation determines directional migration in response to the extracellular matrix. The Journal of cell biology 2007, 177, 527-538, doi:10.1083/jcb.200610076.

37. Pegtel, D.M.; Ellenbroek, S.I.; Mertens, A.E.; van der Kammen, R.A.; de Rooij, J.; Collard, J.G. The Par-Tiam1 complex controls persistent migration by stabilizing microtubule-dependent front-rear polarity. Current biology : CB 2007, 17, 1623-1634, doi:10.1016/j.cub.2007.08.035.

38. Wang, S.; Watanabe, T.; Matsuzawa, K.; Katsumi, A.; Kakeno, M.; Matsui, T.; Ye, F.; Sato, K.; Murase, K.; Sugiyama, I., et al. Tiam1 interaction with the PAR complex promotes talin-mediated Rac1 activation during polarized cell migration. The Journal of cell biology 2012, 199, 331-345, doi:10.1083/jcb.201202041.

39. Pasten, C.; Cerda, J.; Jausoro, I.; Court, F.A.; Caceres, A.; Marzolo, M.P. ApoER2 and Reelin are expressed in regenerating peripheral nerve and regulate Schwann cell migration by activating the Rac1 GEF protein, Tiam1. Molecular and cellular neurosciences 2015, 69, 111, doi:10.1016/j.men.2015.09.004.

40. Wei, C.; Wang, X.; Zheng, M.; Cheng, H. Calcium gradients underlying cell migration. Current opinion in cell biology 2012, 24, 254-261, doi:10.1016/j.ceb.2011.12.002.

41. Horigane, S.I.; Ozawa, Y.; Yamada, H.; Takemoto-Kimura, S. Calcium signalling: a key regulator of neuronal migration. Journal of biochemistry 2019, 165, 401-409, doi:10.1093/jb/mvz012.

42. Saez, P.J.; Saez, J.C.; Lennon-Dumenil, A.M.; Vargas, P. Role of calcium permeable channels in dendritic cell migration. Current opinion in immunology 2018, 52, 74-80, doi:10.1016/j.coi.2018.04.005.

43. Babona-Pilipos, R.; Liu, N.; Pritchard-Oh, A.; Mok, A.; Badawi, D.; Popovic, M.R.; Morshead, C.M. Calcium influx differentially regulates migration velocity and directedness in response to electric field application. Experimental cell research 2018, 368, 202-214, doi:10.1016/j.yexcr.2018.04.031.

44. Xiang, B.; Liu, Y.; Zhao, W.; Zhao, H.; Yu, H. Extracellular calcium regulates the adhesion and migration of osteoclasts via integrin alphav beta 3 /Rho A/Cytoskeleton signaling. Cell biology international 2019, 43, 1125-1136, doi:10.1002/cbin.11033.

45. Tsai, F.C.; Kuo, G.H.; Chang, S.W.; Tsai, P.J. Ca2+ signaling in cytoskeletal reorganization, cell migration, and cancer metastasis. BioMed research international 2015, 2015, 409245, doi:10.1155/2015/409245.

46. Kim, J.M.; Lee, M.; Kim, N.; Heo, W.D. Optogenetic toolkit reveals the role of Ca2+ sparklets in coordinated cell migration. Proceedings of the National Academy of Sciences of the United States of America 2016, 113, 5952-5957, doi:10.1073/pnas.1518412113.

47. Wang, Q.; Chen, M.; Schafer, N.P.; Bueno, C.; Song, S.S.; Hudmon, A.; Wolynes, P.G.; Waxham, M.N.; Cheung, M.S. Assemblies of calcium/calmodulin-dependent kinase II with actin and their dynamic regulation by calmodulin in dendritic spines. Proceedings of the National Academy of Sciences of the United States of America 2019, 116, 18937-18942, doi:10.1073/pnas.1911452116.

48. Wales, P.; Schuberth, C.E.; Aufschnaiter, R.; Fels, J.; Garcia-Aguilar, I.; Janning, A.; Dlugos, C.P.; Schafer-Herte, M.; Klingner, C.; Walte, M., et al. Calcium-mediated actin reset (CaAR) mediates acute cell adaptations. eLife 2016, 5, doi:10.7554/eLife.19850.

49. Merriam, E.B.; Millette, M.; Lumbard, D.C.; Saengsawang, W.; Fothergill, T.; Hu, X.; Ferhat, L.; Dent, E.W. Synaptic regulation of microtubule dynamics in dendritic spines by calcium, F-actin, and drebrin. The Journal of neuroscience : the official journal of the Society for Neuroscience 2013, 33, 16471-16482, doi:10.1523/JNEUROSCI.0661-13.2013.

50. Bernfield, M.; Kokenyesi, R.; Kato, M.; Hinkes, M.T.; Spring, J.; Gallo, R.L.; Lose, 
E.J. Biology of the syndecans: a family of transmembrane heparan sulfate proteoglycans. Annual review of cell biology 1992, 8, 365-393, doi:10.1146/annurev.cb.08.110192.002053.

51. Kim, C.W.; Goldberger, O.A.; Gallo, R.L.; Bernfield, M. Members of the syndecan family of heparan sulfate proteoglycans are expressed in distinct cell-, tissue-, and development-specific patterns. Molecular biology of the cell 1994, 5, 797-805, doi:10.1091/mbc.5.7.797.

52. Yin, H.; Price, F; Rudnicki, M.A. Satellite cells and the muscle stem cell niche. Physiological reviews 2013, 93, 23-67, doi:10.1152/physrev.00043.2011.

53. Carey, D.J. Syndecans: multifunctional cell-surface co-receptors. The Biochemical journal 1997, 327 ( Pt 1), 1-16, doi:10.1042/bj3270001.

54. Langford, J.K.; Stanley, M.J.; Cao, D.; Sanderson, R.D. Multiple heparan sulfate chains are required for optimal syndecan-1 function. The Journal of biological chemistry 1998, 273, 29965-29971, doi:10.1074/jbc.273.45.29965.

55. Fitzgerald, M.L.; Wang, Z.; Park, P.W.; Murphy, G.; Bernfield, M. Shedding of syndecan- 1 and -4 ectodomains is regulated by multiple signaling pathways and mediated by a TIMP-3-sensitive metalloproteinase. The Journal of cell biology 2000, 148, 811-824, doi:10.1083/jcb.148.4.811.

56. Park, P.W.; Foster, T.J.; Nishi, E.; Duncan, S.J.; Klagsbrun, M.; Chen, Y. Activation of syndecan-1 ectodomain shedding by Staphylococcus aureus alpha-toxin and beta-toxin. The Journal of biological chemistry 2004, 279, 251-258, doi:10.1074/jbc.M308537200.

57. Yuan, K.; Hong, T.M.; Chen, J.J.; Tsai, W.H.; Lin, M.T. Syndecan-1 up-regulated by ephrinB2/EphB4 plays dual roles in inflammatory angiogenesis. Blood 2004, 104, 1025-1033, doi:10.1182/blood-2003-09-3334.

58. Chen, D.; Adenekan, B.; Chen, L.; Vaughan, E.D.; Gerald, W.; Feng, Z.; Knudsen, B.S. Syndecan-1 expression in locally invasive and metastatic prostate cancer. Urology 2004, 63, 402-407, doi:10.1016/j.urology.2003.08.036.

59. Adepu, S.; Rosman, C.W.; Dam, W.; van Dijk, M.C.; Navis, G.; van Goor, H.; Bakker, S.J.; van den Born, J. Incipient renal transplant dysfunction associates with tubular syndecan1 expression and shedding. American journal of physiology. Renal physiology 2015, 309, F137-145, doi:10.1152/ajprenal.00127.2015.

60. Vuong, T.T.; Reine, T.M.; Sudworth, A.; Jenssen, T.G.; Kolset, S.O. Syndecan-4 is a major syndecan in primary human endothelial cells in vitro, modulated by inflammatory stimuli and involved in wound healing. The journal of histochemistry and cytochemistry : official journal of the Histochemistry Society 2015, 63, 280-292, doi:10.1369/0022155415568995.

61. Manon-Jensen, T.; Itoh, Y.; Couchman, J.R. Proteoglycans in health and disease: the multiple roles of syndecan shedding. The FEBS journal 2010, 277, 3876-3889, doi:10.1111/j.1742-4658.2010.07798.x.

62. Choi, S.; Lee, E.; Kwon, S.; Park, H.; Yi, J.Y.; Kim, S.; Han, I.O.; Yun, Y.; Oh, E.S. Transmembrane domain-induced oligomerization is crucial for the functions of syndecan-2 and syndecan-4. The Journal of biological chemistry 2005, 280, 42573-42579, doi:10.1074/jbc.M509238200.

63. Dews, I.C.; Mackenzie, K.R. Transmembrane domains of the syndecan family of growth factor coreceptors display a hierarchy of homotypic and heterotypic interactions. Proceedings of the National Academy of Sciences of the United States of America 2007, 104, 20782-20787, doi:10.1073/pnas.0708909105.

64. Couchman, J.R. Syndecans: proteoglycan regulators of cell-surface microdomains? Nature reviews. Molecular cell biology 2003, 4, 926-937, doi:10.1038/nrm1257.

65. Couchman, J.R.; Chen, L.; Woods, A. Syndecans and cell adhesion. International review of cytology 2001, 207, 113-150, doi:10.1016/s0074-7696(01)07004-8. 
66. Granes, F.; Berndt, C.; Roy, C.; Mangeat, P.; Reina, M.; Vilaro, S. Identification of a novel Ezrin-binding site in syndecan-2 cytoplasmic domain. FEBS letters 2003, 547, 212-216, doi:10.1016/s0014-5793(03)00712-9.

67. Choi, Y.; Kim, S.; Lee, J.; Ko, S.G.; Lee, W.; Han, I.O.; Woods, A.; Oh, E.S. The oligomeric status of syndecan-4 regulates syndecan-4 interaction with alpha-actinin. European journal of cell biology 2008, 87, 807-815, doi:10.1016/j.ejcb.2008.04.005.

68. Greene, D.K.; Tumova, S.; Couchman, J.R.; Woods, A. Syndecan-4 associates with alpha-actinin. The Journal of biological chemistry 2003, 278, 7617-7623, doi:10.1074/jbc.M207123200.

69. Multhaupt, H.A.; Yoneda, A.; Whiteford, J.R.; Oh, E.S.; Lee, W.; Couchman, J.R. Syndecan signaling: when, where and why? Journal of physiology and pharmacology : an official journal of the Polish Physiological Society 2009, 60 Suppl 4, 31-38.

70. Leblanc, R.; Sahay, D.; Houssin, A.; Machuca-Gayet, I.; Peyruchaud, O. Autotaxinbeta interaction with the cell surface via syndecan-4 impacts on cancer cell proliferation and metastasis. Oncotarget 2018, 9, 33170-33185, doi:10.18632/oncotarget.26039.

71. Becsky, D.; Gyulai-Nagy, S.; Balind, A.; Horvath, P.; Dux, L.; Keller-Pinter, A. Myoblast Migration and Directional Persistence Affected by Syndecan-4-Mediated Tiam-1 Expression and Distribution. International journal of molecular sciences 2020, 21, doi:10.3390/ijms21030823.

72. Gopal, S.; Multhaupt, H.A.B.; Pocock, R.; Couchman, J.R. Cell-extracellular matrix and cell-cell adhesion are linked by syndecan-4. Matrix biology : journal of the International Society for Matrix Biology 2017, 60-61, 57-69, doi:10.1016/j.matbio.2016.10.006.

73. Elfenbein, A.; Simons, M. Syndecan-4 signaling at a glance. Journal of cell science 2013, 126, 3799-3804, doi:10.1242/jcs.124636.

74. Kawaguchi, Y.; Takeuchi, T.; Kuwata, K.; Chiba, J.; Hatanaka, Y.; Nakase, I.; Futaki, S. Syndecan-4 Is a Receptor for Clathrin-Mediated Endocytosis of Arginine-Rich CellPenetrating Peptides. Bioconjugate chemistry 2016, 27, 1119-1130, doi:10.1021/acs.bioconjchem.6b00082.

75. Bellin, R.M.; Kubicek, J.D.; Frigault, M.J.; Kamien, A.J.; Steward, R.L., Jr.; Barnes, H.M.; Digiacomo, M.B.; Duncan, L.J.; Edgerly, C.K.; Morse, E.M., et al. Defining the role of syndecan-4 in mechanotransduction using surface-modification approaches. Proceedings of the National Academy of Sciences of the United States of America 2009, 106, 22102-22107, doi:10.1073/pnas.0902639106.

76. Lambert, J.; Makin, K.; Akbareian, S.; Johnson, R.; Alghamdi, A.A.A.; Robinson, S.D.; Edwards, D.R. ADAMTS-1 and syndecan-4 intersect in the regulation of cell migration and angiogenesis. Journal of cell science 2020, 133, doi:10.1242/jcs.235762.

77. Baeyens, N.; Mulligan-Kehoe, M.J.; Corti, F.; Simon, D.D.; Ross, T.D.; Rhodes, J.M.; Wang, T.Z.; Mejean, C.O.; Simons, M.; Humphrey, J., et al. Syndecan 4 is required for endothelial alignment in flow and atheroprotective signaling. Proceedings of the National Academy of Sciences of the United States of America 2014, 111, 17308-17313, doi:10.1073/pnas.1413725111.

78. Iwabuchi, T.; Goetinck, P.F. Syndecan-4 dependent FGF stimulation of mouse vibrissae growth. Mechanisms of development 2006, 123, 831-841, doi:10.1016/j.mod.2006.08.003.

79. Borland, S.J.; Morris, T.G.; Borland, S.C.; Morgan, M.R.; Francis, S.E.; Merry, C.L.R.; Canfield, A.E. Regulation of vascular smooth muscle cell calcification by syndecan4/FGF-2/PKCalpha signalling and cross-talk with TGFbeta. Cardiovascular research 2017, 113, 1639-1652, doi:10.1093/cvr/cvx178.

80. Das, S.; Majid, M.; Baker, A.B. Syndecan-4 enhances PDGF-BB activity in diabetic wound healing. Acta biomaterialia 2016, 42, 56-65, doi:10.1016/j.actbio.2016.07.001. 
81. Tumova, S.; Woods, A.; Couchman, J.R. Heparan sulfate chains from glypican and syndecans bind the Hep II domain of fibronectin similarly despite minor structural differences. The Journal of biological chemistry 2000, 275, 9410-9417, doi:10.1074/jbc.275.13.9410.

82. Woods, A.; Couchman, J.R. Syndecan-4 and focal adhesion function. Current opinion in cell biology 2001, 13, 578-583, doi:10.1016/s0955-0674(00)00254-4.

83. Bass, M.D.; Humphries, M.J. Cytoplasmic interactions of syndecan-4 orchestrate adhesion receptor and growth factor receptor signalling. The Biochemical journal 2002, 368, 1-15, doi:10.1042/BJ20021228.

84. Baciu, P.C.; Goetinck, P.F. Protein kinase C regulates the recruitment of syndecan-4 into focal contacts. Molecular biology of the cell 1995, 6, 1503-1513, doi:10.1091/mbc.6.11.1503.

85. Tkachenko, E.; Simons, M. Clustering induces redistribution of syndecan-4 core protein into raft membrane domains. The Journal of biological chemistry 2002, 277, 1994619951, doi:10.1074/jbc.M200841200.

86. Horowitz, A.; Simons, M. Regulation of syndecan-4 phosphorylation in vivo. The Journal of biological chemistry 1998, 273, 10914-10918, doi:10.1074/jbc.273.18.10914.

87. Couchman, J.R.; Vogt, S.; Lim, S.T.; Lim, Y.; Oh, E.S.; Prestwich, G.D.; Theibert, A.; Lee, W.; Woods, A. Regulation of inositol phospholipid binding and signaling through syndecan-4. The Journal of biological chemistry 2002, 277, 49296-49303, doi:10.1074/jbc.M209679200.

88. Koo, B.K.; Jung, Y.S.; Shin, J.; Han, I.; Mortier, E.; Zimmermann, P.; Whiteford, J.R.; Couchman, J.R.; Oh, E.S.; Lee, W. Structural basis of syndecan-4 phosphorylation as a molecular switch to regulate signaling. Journal of molecular biology 2006, 355, 651-663, doi:10.1016/j.jmb.2005.09.087.

89. Oh, E.S.; Woods, A.; Couchman, J.R. Syndecan-4 proteoglycan regulates the distribution and activity of protein kinase C. The Journal of biological chemistry 1997, 272, 8133-8136, doi:10.1074/jbc.272.13.8133.

90. Roper, J.A.; Williamson, R.C.; Bass, M.D. Syndecan and integrin interactomes: large complexes in small spaces. Current opinion in structural biology 2012, 22, 583-590, doi:10.1016/j.sbi.2012.07.003.

91. Afratis, N.A.; Nikitovic, D.; Multhaupt, H.A.; Theocharis, A.D.; Couchman, J.R.; Karamanos, N.K. Syndecans - key regulators of cell signaling and biological functions. The FEBS journal 2017, 284, 27-41, doi:10.1111/febs.13940.

92. Finsen, A.V.; Lunde, I.G.; Sjaastad, I.; Ostli, E.K.; Lyngra, M.; Jarstadmarken, H.O.; Hasic, A.; Nygard, S.; Wilcox-Adelman, S.A.; Goetinck, P.F., et al. Syndecan-4 is essential for development of concentric myocardial hypertrophy via stretch-induced activation of the calcineurin-NFAT pathway. PloS one 2011, 6, e28302, doi:10.1371/journal.pone.0028302.

93. Tkachenko, E.; Lutgens, E.; Stan, R.V.; Simons, M. Fibroblast growth factor 2 endocytosis in endothelial cells proceed via syndecan-4-dependent activation of Rac1 and a Cdc42-dependent macropinocytic pathway. Journal of cell science 2004, 117, 3189-3199, doi:10.1242/jcs.01190.

94. Liu, X.; Shepherd, T.R.; Murray, A.M.; Xu, Z.; Fuentes, E.J. The structure of the Tiam1 PDZ domain/ phospho-syndecan1 complex reveals a ligand conformation that modulates protein dynamics. Structure 2013, 21, 342-354, doi:10.1016/j.str.2013.01.004.

95. Shepherd, T.R.; Klaus, S.M.; Liu, X.; Ramaswamy, S.; DeMali, K.A.; Fuentes, E.J. The Tiam1 PDZ domain couples to Syndecan1 and promotes cell-matrix adhesion. Journal of molecular biology 2010, 398, 730-746, doi:10.1016/j.jmb.2010.03.047.

96. Keller-Pinter, A.; Ughy, B.; Domoki, M.; Pettko-Szandtner, A.; Letoha, T.; Tovari, J.; Timar, J.; Szilak, L. The phosphomimetic mutation of syndecan-4 binds and inhibits Tiam1 
modulating Rac1 activity in PDZ interaction-dependent manner. PloS one 2017, 12, e0187094, doi:10.1371/journal.pone.0187094.

97. Gopal, S.; Sogaard, P.; Multhaupt, H.A.; Pataki, C.; Okina, E.; Xian, X.; Pedersen, M.E.; Stevens, T.; Griesbeck, O.; Park, P.W., et al. Transmembrane proteoglycans control stretch-activated channels to set cytosolic calcium levels. The Journal of cell biology 2015, 210, 1199-1211, doi:10.1083/jcb.201501060.

98. Liu, Y.; Echtermeyer, F.; Thilo, F.; Theilmeier, G.; Schmidt, A.; Schulein, R.; Jensen, B.L.; Loddenkemper, C.; Jankowski, V.; Marcussen, N., et al. The proteoglycan syndecan 4 regulates transient receptor potential canonical 6 channels via RhoA/Rho-associated protein kinase signaling. Arteriosclerosis, thrombosis, and vascular biology 2012, 32, 378-385, doi:10.1161/ATVBAHA.111.241018.

99. Kainulainen, V.; Wang, H.; Schick, C.; Bernfield, M. Syndecans, heparan sulfate proteoglycans, maintain the proteolytic balance of acute wound fluids. The Journal of biological chemistry 1998, 273, 11563-11569, doi:10.1074/jbc.273.19.11563.

100. Bass, M.D.; Williamson, R.C.; Nunan, R.D.; Humphries, J.D.; Byron, A.; Morgan, M.R.; Martin, P.; Humphries, M.J. A syndecan-4 hair trigger initiates wound healing through caveolin- and RhoG-regulated integrin endocytosis. Developmental cell 2011, 21, 681-693, doi:10.1016/j.devcel.2011.08.007.

101. Reizes, O.; Lincecum, J.; Wang, Z.; Goldberger, O.; Huang, L.; Kaksonen, M.; Ahima, R.; Hinkes, M.T.; Barsh, G.S.; Rauvala, H., et al. Transgenic expression of syndecan-1 uncovers a physiological control of feeding behavior by syndecan-3. Cell 2001, 106, 105-116, doi:10.1016/s0092-8674(01)00415-9.

102. Cornelison, D.D.; Wilcox-Adelman, S.A.; Goetinck, P.F.; Rauvala, H.; Rapraeger, A.C.; Olwin, B.B. Essential and separable roles for Syndecan-3 and Syndecan-4 in skeletal muscle development and regeneration. Genes \& development 2004, 18, 2231-2236, doi:10.1101/gad.1214204.

103. Echtermeyer, F.; Streit, M.; Wilcox-Adelman, S.; Saoncella, S.; Denhez, F.; Detmar, M.; Goetinck, P. Delayed wound repair and impaired angiogenesis in mice lacking syndecan4. The Journal of clinical investigation 2001, 107, R9-R14, doi:10.1172/JCI10559.

104. Stepp, M.A.; Gibson, H.E.; Gala, P.H.; Iglesia, D.D.; Pajoohesh-Ganji, A.; Pal-Ghosh, S.; Brown, M.; Aquino, C.; Schwartz, A.M.; Goldberger, O., et al. Defects in keratinocyte activation during wound healing in the syndecan-1-deficient mouse. Journal of cell science 2002, 115, 4517-4531, doi:10.1242/jcs.00128.

105. Piccinini, F.; Kiss, A.; Horvath, P. CellTracker (not only) for dummies. Bioinformatics 2016, 32, 955-957, doi:10.1093/bioinformatics/btv686.

106. Gorelik, R.; Gautreau, A. Quantitative and unbiased analysis of directional persistence in cell migration. Nature protocols 2014, 9, 1931-1943, doi:10.1038/nprot.2014.131.

107. Etienne-Manneville, S.; Hall, A. Integrin-mediated activation of Cdc42 controls cell polarity in migrating astrocytes through PKCzeta. Cell 2001, 106, 489-498, doi:10.1016/s0092-8674(01)00471-8.

108. van de Linde, S.; Loschberger, A.; Klein, T.; Heidbreder, M.; Wolter, S.; Heilemann, M.; Sauer, M. Direct stochastic optical reconstruction microscopy with standard fluorescent probes. Nature protocols 2011, 6, 991-1009, doi:10.1038/nprot.2011.336.

109. Rees, E.J.; Erdelyi, M.; Schierle, G.S.K.; Knight, A.; Kaminski, C.F. Elements of image processing in localization microscopy. Journal of Optics 2013, 15, 094012, doi:10.1088/2040-8978/15/9/094012.

110. Thompson, R.E.; Larson, D.R.; Webb, W.W. Precise nanometer localization analysis for individual fluorescent probes. Biophysical journal 2002, 82, 2775-2783, doi:10.1016/S0006-3495(02)75618-X.

111. Liang, C.C.; Park, A.Y.; Guan, J.L. In vitro scratch assay: a convenient and 
inexpensive method for analysis of cell migration in vitro. Nature protocols $\mathbf{2 0 0 7}, 2$, 329-333, doi:10.1038/nprot.2007.30.

112. Barriga, E.H.; Shellard, A.; Mayor, R. In Vivo and In Vitro Quantitative Analysis of Neural Crest Cell Migration. Methods in molecular biology 2019, 1976, 135-152, doi:10.1007/978-1-4939-9412-0_11.

113. Xu, F.; Qiao, L.; Zhao, Y.; Chen, W.; Hong, S.; Pan, J.; Jiang, B. The potential application of concentrated growth factor in pulp regeneration: an in vitro and in vivo study. Stem cell research \& therapy 2019, 10, 134, doi:10.1186/s13287-019-1247-4.

114. Martinotti, S.; Ranzato, E. Scratch Wound Healing Assay. Methods in molecular biology 2020, 2109, 225-229, doi:10.1007/7651_2019_259.

115. Rodriguez, L.G.; Wu, X.; Guan, J.L. Wound-healing assay. Methods in molecular biology 2005, 294, 23-29, doi:10.1385/1-59259-860-9:023.

116. Giger, F.A.; Dumortier, J.G.; David, N.B. Analyzing In Vivo Cell Migration using Cell Transplantations and Time-lapse Imaging in Zebrafish Embryos. Journal of visualized experiments : JoVE 2016, 10.3791/53792, doi:10.3791/53792.

117. Geris, L. Regenerative orthopaedics: in vitro, in vivo...in silico. International orthopaedics 2014, 38, 1771-1778, doi:10.1007/s00264-014-2419-6.

118. Lucas, L.C.; Dale, P.; Buchanan, R.; Gill, Y.; Griffin, D.; Lemons, J.E. In vitro vs in vivo corrosion analyses of two alloys. Journal of investigative surgery : the official journal of the Academy of Surgical Research 1991, 4, 13-21, doi:10.3109/08941939109140757.

119. Ghosh, P.; Milewski, M.; Paudel, K. In vitro/in vivo correlations in transdermal product development. Therapeutic delivery 2015, 6, 1117-1124, doi:10.4155/tde.15.72.

120. Newman, S.P.; Chan, H.K. In vitro/in vivo comparisons in pulmonary drug delivery. Journal of aerosol medicine and pulmonary drug delivery 2008, 21, 77-84, doi:10.1089/jamp.2007.0643.

121. Baciu, P.C.; Saoncella, S.; Lee, S.H.; Denhez, F.; Leuthardt, D.; Goetinck, P.F. Syndesmos, a protein that interacts with the cytoplasmic domain of syndecan-4, mediates cell spreading and actin cytoskeletal organization. Journal of cell science 2000, 113 Pt 2, 315-324. 122. Cavalheiro, R.P.; Lima, M.A.; Jarrouge-Boucas, T.R.; Viana, G.M.; Lopes, C.C.; Coulson-Thomas, V.J.; Dreyfuss, J.L.; Yates, E.A.; Tersariol, I.L.S.; Nader, H.B. Coupling of vinculin to F-actin demands Syndecan-4 proteoglycan. Matrix biology : journal of the International Society for Matrix Biology 2017, 63, 23-37, doi:10.1016/j.matbio.2016.12.006.

123. Vuoriluoto, K.; Jokinen, J.; Kallio, K.; Salmivirta, M.; Heino, J.; Ivaska, J. Syndecan-1 supports integrin alpha2beta1-mediated adhesion to collagen. Experimental cell research 2008, 314, 3369-3381, doi:10.1016/j.yexcr.2008.07.005.

124. Lauffenburger, D.A.; Horwitz, A.F. Cell migration: a physically integrated molecular process. Cell 1996, 84, 359-369, doi:10.1016/s0092-8674(00)81280-5.

125. Petrie, R.J.; Zhao, B.; Bedford, F.; Lamarche-Vane, N. Compartmentalized DCC signalling is distinct from DCC localized to lipid rafts. Biology of the cell 2009, 101, 77-90, doi:10.1042/BC20070108.

126. Gardel, M.L.; Schneider, I.C.; Aratyn-Schaus, Y.; Waterman, C.M. Mechanical integration of actin and adhesion dynamics in cell migration. Annual review of cell and developmental biology 2010, 26, 315-333, doi:10.1146/annurev.cellbio.011209.122036.

127. Parsons, J.T.; Horwitz, A.R.; Schwartz, M.A. Cell adhesion: integrating cytoskeletal dynamics and cellular tension. Nature reviews. Molecular cell biology 2010, 11, 633-643, doi:10.1038/nrm2957.

128. Devendran, C.; Carthew, J.; Frith, J.E.; Neild, A. Cell Adhesion, Morphology, and Metabolism Variation via Acoustic Exposure within Microfluidic Cell Handling Systems. Advanced science 2019, 6, 1902326, doi:10.1002/advs.201902326.

129. Zhang, J.; Wang, Y.L. Centrosome defines the rear of cells during mesenchymal 
migration. Molecular biology of the cell 2017, 28, 3240-3251, doi:10.1091/mbc.E17-06-0366. 130. Gotlieb, A.I.; Subrahmanyan, L.; Kalnins, V.I. Microtubule-organizing centers and cell migration: effect of inhibition of migration and microtubule disruption in endothelial cells. The Journal of cell biology 1983, 96, 1266-1272, doi:10.1083/jcb.96.5.1266.

131. Keller-Pinter, A.; Bottka, S.; Timar, J.; Kulka, J.; Katona, R.; Dux, L.; Deak, F.; Szilak, L. Syndecan-4 promotes cytokinesis in a phosphorylation-dependent manner. Cellular and molecular life sciences : CMLS 2010, 67, 1881-1894, doi:10.1007/s00018-010-0298-6.

132. Thomas, D.; Tovey, S.C.; Collins, T.J.; Bootman, M.D.; Berridge, M.J.; Lipp, P. A comparison of fluorescent $\mathrm{Ca} 2+$ indicator properties and their use in measuring elementary and global Ca2+ signals. Cell calcium 2000, 28, 213-223, doi:10.1054/ceca.2000.0152.

133. Brooks, R.; Williamson, R.; Bass, M. Syndecan-4 independently regulates multiple small GTPases to promote fibroblast migration during wound healing. Small GTPases 2012, 3, 73-79, doi:10.4161/sgtp.19301.

134. Saoncella, S.; Calautti, E.; Neveu, W.; Goetinck, P.F. Syndecan-4 regulates ATF-2 transcriptional activity in a Rac1-dependent manner. The Journal of biological chemistry 2004, 279, 47172-47176, doi:10.1074/jbc.C400299200.

135. Matthews, H.K.; Marchant, L.; Carmona-Fontaine, C.; Kuriyama, S.; Larrain, J.; Holt, M.R.; Parsons, M.; Mayor, R. Directional migration of neural crest cells in vivo is regulated by Syndecan-4/Rac1 and non-canonical Wnt signaling/RhoA. Development 2008, 135, 17711780, doi:10.1242/dev.017350.

136. Gao, Y.; Dickerson, J.B.; Guo, F.; Zheng, J.; Zheng, Y. Rational design and characterization of a Rac GTPase-specific small molecule inhibitor. Proceedings of the National Academy of Sciences of the United States of America 2004, 101, 7618-7623, doi:10.1073/pnas.0307512101.

137. Karalaki, M.; Fili, S.; Philippou, A.; Koutsilieris, M. Muscle regeneration: cellular and molecular events. In vivo 2009, 23, 779-796.

138. Otrocka-Domagala, I.; Pazdzior-Czapula, K.; Maslanka, T. Simvastatin Impairs the Inflammatory and Repair Phases of the Postinjury Skeletal Muscle Regeneration. BioMed research international 2018, 2018, 7617312, doi:10.1155/2018/7617312.

139. Chung, H.; Multhaupt, H.A.; Oh, E.S.; Couchman, J.R. Minireview: Syndecans and their crucial roles during tissue regeneration. FEBS letters 2016, 590, 2408-2417, doi:10.1002/1873-3468.12280.

140. Keller-Pinter, A.; Szabo, K.; Kocsis, T.; Deak, F.; Ocsovszki, I.; Zvara, A.; Puskas, L.; Szilak, L.; Dux, L. Syndecan-4 influences mammalian myoblast proliferation by modulating myostatin signalling and G1/S transition. FEBS letters 2018, 592, 3139-3151, doi:10.1002/1873-3468.13227.

141. Yin, L.; Qi, Y.; Xu, Y.; Xu, L.; Han, X.; Tao, X.; Song, S.; Peng, J. Dioscin Inhibits HSC-T6 Cell Migration via Adjusting SDC-4 Expression: Insights from iTRAQ-Based Quantitative Proteomics. Frontiers in pharmacology 2017, 8, 665, doi:10.3389/fphar.2017.00665.

142. Qin, Y.; Zhu, Y.; Luo, F.; Chen, C.; Chen, X.; Wu, M. Killing two birds with one stone: dual blockade of integrin and FGF signaling through targeting syndecan-4 in postoperative capsular opacification. Cell death \& disease 2017, 8, e2920, doi:10.1038/cddis.2017.315.

143. Li, R.; Wu, H.; Xie, J.; Li, G.; Gu, R.; Kang, L.; Wang, L.; Xu, B. Syndecan-4 regulates the bFGF-induced chemotactic migration of endothelial cells. Journal of molecular histology 2016, 47, 503-509, doi:10.1007/s10735-016-9693-0.

144. Lin, F.; Ren, X.D.; Doris, G.; Clark, R.A. Three-dimensional migration of human adult dermal fibroblasts from collagen lattices into fibrin/fibronectin gels requires syndecan-4 proteoglycan. The Journal of investigative dermatology 2005, 124, 906-913, doi:10.1111/j.0022-202X.2005.23740.x. 
145. Averbeck, M.; Gebhardt, C.; Anderegg, U.; Termeer, C.; Sleeman, J.P.; Simon, J.C. Switch in syndecan-1 and syndecan-4 expression controls maturation associated dendritic cell motility. Experimental dermatology 2007, 16, 580-589, doi:10.1111/j.16000625.2007.00568.x.

146. Toba-Ichihashi, Y.; Yamaoka, T.; Ohmori, T.; Ohba, M. Up-regulation of Syndecan-4 contributes to TGF-beta1-induced epithelial to mesenchymal transition in lung adenocarcinoma A549 cells. Biochemistry and biophysics reports 2016, 5, 1-7, doi:10.1016/j.bbrep.2015.11.021.

147. Charni, F.; Friand, V.; Haddad, O.; Hlawaty, H.; Martin, L.; Vassy, R.; Oudar, O.; Gattegno, L.; Charnaux, N.; Sutton, A. Syndecan-1 and syndecan-4 are involved in RANTES/CCL5-induced migration and invasion of human hepatoma cells. Biochimica et biophysica acta 2009, 1790, 1314-1326, doi:10.1016/j.bbagen.2009.07.015.

148. Polte, T.; Petzold, S.; Bertrand, J.; Schutze, N.; Hinz, D.; Simon, J.C.; Lehmann, I.; Echtermeyer, F.; Pap, T.; Averbeck, M. Critical role for syndecan-4 in dendritic cell migration during development of allergic airway inflammation. Nature communications 2015, 6, 7554, doi:10.1038/ncomms8554.

149. Endo, T.; Ito, K.; Morimoto, J.; Kanayama, M.; Ota, D.; Ikesue, M.; Kon, S.; Takahashi, D.; Onodera, T.; Iwasaki, N., et al. Syndecan 4 Regulation of the Development of Autoimmune Arthritis in Mice by Modulating B Cell Migration and Germinal Center Formation. Arthritis \& rheumatology 2015, 67, 2512-2522, doi:10.1002/art.39193.

150. Jeyarajah, M.J.; Jaju Bhattad, G.; Kops, B.F.; Renaud, S.J. Syndecan-4 regulates extravillous trophoblast migration by coordinating protein kinase $\mathrm{C}$ activation. Scientific reports 2019, 9, 10175, doi:10.1038/s41598-019-46599-6.

151. Sanderson, R.D.; Yang, Y.; Suva, L.J.; Kelly, T. Heparan sulfate proteoglycans and heparanase--partners in osteolytic tumor growth and metastasis. Matrix biology : journal of the International Society for Matrix Biology 2004, 23, 341-352, doi:10.1016/j.matbio.2004.08.004.

152. Okolicsanyi, R.K.; Buffiere, A.; Jacinto, J.M.; Chacon-Cortes, D.; Chambers, S.K.; Youl, P.H.; Haupt, L.M.; Griffiths, L.R. Association of heparan sulfate proteoglycans SDC1 and SDC4 polymorphisms with breast cancer in an Australian Caucasian population. Tumour biology : the journal of the International Society for Oncodevelopmental Biology and Medicine 2015, 36, 1731-1738, doi:10.1007/s13277-014-2774-3.

153. Yang, L.; Zhang, X.; Hou, Q.; Huang, M.; Zhang, H.; Jiang, Z.; Yue, J.; Wu, S. Singlecell RNA-seq of esophageal squamous cell carcinoma cell line with fractionated irradiation reveals radioresistant gene expression patterns. BMC genomics 2019, 20, 611, doi:10.1186/s12864-019-5970-0.

154. Kim, J.H.; Park, S.C. Syndecan-2 Methylation as a New Biomarker for Early Detection of Colorectal Neoplasm. Gut and liver 2018, 12, 479-480, doi:10.5009/gnl18286.

155. Cui, J.; Jin, S.; Jin, C.; Jin, Z. Syndecan-1 regulates extracellular matrix expression in keloid fibroblasts via TGF-beta1/Smad and MAPK signaling pathways. Life sciences 2020, 254, 117326, doi:10.1016/j.lfs.2020.117326.

156. Reyes, I.; Reyes, N.; Suriano, R.; Iacob, C.; Suslina, N.; Policastro, A.; Moscatello, A.; Schantz, S.; Tiwari, R.K.; Geliebter, J. Gene expression profiling identifies potential molecular markers of papillary thyroid carcinoma. Cancer biomarkers : section A of Disease markers 2019, 24, 71-83, doi:10.3233/CBM-181758.

157. Huang, W.; Chiquet-Ehrismann, R.; Moyano, J.V.; Garcia-Pardo, A.; Orend, G. Interference of tenascin- $\mathrm{C}$ with syndecan- 4 binding to fibronectin blocks cell adhesion and stimulates tumor cell proliferation. Cancer research 2001, 61, 8586-8594.

158. Na, K.Y.; Bacchini, P.; Bertoni, F.; Kim, Y.W.; Park, Y.K. Syndecan-4 and fibronectin in osteosarcoma. Pathology 2012, 44, 325-330, doi:10.1097/PAT.0b013e328353447b. 
159. Hall, A. Rho GTPases and the actin cytoskeleton. Science 1998, 279, 509-514, doi:10.1126/science.279.5350.509.

160. Burridge, K. Crosstalk between Rac and Rho. Science 1999, 283, 2028-2029, doi:10.1126/science.283.5410.2028.

161. Rottner, K.; Hall, A.; Small, J.V. Interplay between Rac and Rho in the control of substrate contact dynamics. Current biology : $C B$ 1999, 9, 640-648, doi:10.1016/s09609822(99)80286-3.

162. Chaudhuri, P.; Colles, S.M.; Fox, P.L.; Graham, L.M. Protein kinase Cdelta-dependent phosphorylation of syndecan-4 regulates cell migration. Circulation research 2005, 97, 674681, doi:10.1161/01.RES.0000184667.82354.b1.

163. Yoo, J.; Jeong, M.J.; Cho, H.J.; Oh, E.S.; Han, M.Y. Dynamin II interacts with syndecan-4, a regulator of focal adhesion and stress-fiber formation. Biochemical and biophysical research communications 2005, 328, 424-431, doi:10.1016/j.bbrc.2004.12.179.

164. Wilcox-Adelman, S.A.; Denhez, F.; Goetinck, P.F. Syndecan-4 modulates focal adhesion kinase phosphorylation. The Journal of biological chemistry 2002, 277, 3297032977, doi:10.1074/jbc.M201283200.

165. Swaney, J.S.; Patel, H.H.; Yokoyama, U.; Head, B.P.; Roth, D.M.; Insel, P.A. Focal adhesions in (myo)fibroblasts scaffold adenylyl cyclase with phosphorylated caveolin. The Journal of biological chemistry 2006, 281, 17173-17179, doi:10.1074/jbc.M513097200.

166. Gonzalez Malagon, S.G.; Lopez Munoz, A.M.; Doro, D.; Bolger, T.G.; Poon, E.; Tucker, E.R.; Adel Al-Lami, H.; Krause, M.; Phiel, C.J.; Chesler, L., et al. Glycogen synthase kinase 3 controls migration of the neural crest lineage in mouse and Xenopus. Nature communications 2018, 9, 1126, doi:10.1038/s41467-018-03512-5.

167. Elric, J.; Etienne-Manneville, S. Centrosome positioning in polarized cells: common themes and variations. Experimental cell research 2014, 328, 240-248, doi:10.1016/j.yexcr.2014.09.004.

168. Pankov, R.; Endo, Y.; Even-Ram, S.; Araki, M.; Clark, K.; Cukierman, E.; Matsumoto, K.; Yamada, K.M. A Rac switch regulates random versus directionally persistent cell migration. The Journal of cell biology 2005, 170, 793-802, doi:10.1083/jcb.200503152.

169. Adams, H.C., 3rd; Chen, R.; Liu, Z.; Whitehead, I.P. Regulation of breast cancer cell motility by T-cell lymphoma invasion and metastasis-inducing protein. Breast cancer research : BCR 2010, 12, R69, doi:10.1186/bcr2637.

170. Ding, J.; Yang, F.; Wu, W. Tiam1 high expression is associated with poor prognosis in solid cancers: A meta-analysis. Medicine 2019, 98, e17529, doi:10.1097/MD.0000000000017529.

171. Bonucci, E. Fine structure of early cartilage calcification. Journal of ultrastructure research 1967, 20, 33-50, doi:10.1016/s0022-5320(67)80034-0.

172. Anderson, H.C. Vesicles associated with calcification in the matrix of epiphyseal cartilage. The Journal of cell biology 1969, 41, 59-72, doi:10.1083/jcb.41.1.59.

173. Trams, E.G.; Lauter, C.J.; Salem, N., Jr.; Heine, U. Exfoliation of membrane ectoenzymes in the form of micro-vesicles. Biochimica et biophysica acta 1981, 645, 63-70, doi:10.1016/0005-2736(81)90512-5.

174. Thery, C.; Zitvogel, L.; Amigorena, S. Exosomes: composition, biogenesis and function. Nature reviews. Immunology 2002, 2, 569-579, doi:10.1038/nri855.

175. Sung, B.H.; von Lersner, A.; Guerrero, J.; Krystofiak, E.S.; Inman, D.; Pelletier, R.; Zijlstra, A.; Ponik, S.M.; Weaver, A.M. A live cell reporter of exosome secretion and uptake reveals pathfinding behavior of migrating cells. Nature communications 2020, 11, 2092, doi:10.1038/s41467-020-15747-2.

176. Sung, B.H.; Weaver, A.M. Directed migration: Cells navigate by extracellular vesicles. The Journal of cell biology 2018, 217, 2613-2614, doi:10.1083/jcb.201806018. 
177. Sung, B.H.; Ketova, T.; Hoshino, D.; Zijlstra, A.; Weaver, A.M. Directional cell movement through tissues is controlled by exosome secretion. Nature communications 2015, 6, 7164, doi:10.1038/ncomms8164.

178. Baietti, M.F.; Zhang, Z.; Mortier, E.; Melchior, A.; Degeest, G.; Geeraerts, A.; Ivarsson, Y.; Depoortere, F.; Coomans, C.; Vermeiren, E., et al. Syndecan-syntenin-ALIX regulates the biogenesis of exosomes. Nature cell biology 2012, 14, 677-685, doi:10.1038/ncb2502. 


\section{ACKNOWLEDGEMENTS}

"Some people listen to themselves rather than listen to what others say. These people do not come along very often. But when they do, they remind us that once you set up on a path, even though critics may doubt you, it is okay to believe that there is no "can 't", "won't" or "impossible". They remind us that it's okay to believe that impossible is nothing. "

I would like to express my deepest gratitude to those who have supported me during my scientific work. To begin, I would like to express my gratitude to Professor Dr. László Dux and Dr. Tamás Csont for allowing me to work at the Institute of Biochemistry.

I would like to thank my supervisor, Dr. Anikó Keller-Pintér, for accompanying me through these years which were often full of difficulties and challenging tasks, with her perseverance, expertise, and enthusiasm.

I would like to thank Árpád Bálind, Péter Horváth, Zsuzsa Bartos, László Homolya, and Tamás Gajdos, as well as Miklós Erdélyi, for allowing me to conduct live-cell microscopy, $\mathrm{Ca}^{2+}$ level measurements and analyses, also dSTORM measurements.

I would like to thank my colleagues, Erzsébet Rádi, Zita Makráné Felhő, Kitti Szabó, Szuzina Gyulai-Nagy and Zoltán Márton Köhler, for their persistent support and selfless work.

I owe my success to the educators and teachers who have driven me thus far, Dr. Mónika Kiricsi, István Győri, István Csigér.

I'd like to express my heartfelt gratitude to my family in particular. My mother, Eszter Becsky, my father, Balázs Becsky Sr., and my brother, Balázs Becsky Jr. for their unwavering love and support throughout my life. I would like to express my gratitude to my cousin, Dr. Krisztina Boros, for her encouragement, commitment, and advice during the early stages of my study. I would not be where I am without them.

I am much obliged to my partner, Monika Dukić for her persistent support and endless love.

Last but not least, I would like to thank my friends, I could not have surrounded myself with more talented, motivating and intelligent people: Dr. Ágnes Szalenko-Tőkés, Benedikta Balla, Eszter Barna, Dr. Tekla Sáry, Ádám Talmácsi, Dr. Ákos Szabó, Benedek Bodzán, Dr. Benedek Sisák, Dávid Varga, Gábor Szabó, Dr. Gergely Horváth, Dr, Géza Thury, Dr. jur. Konrád Kreiniker, Dr. Kristóf Bozó, Dr. László Kovács, Marko Rafajlović, Dr. Máté Tajti, Máté Vas, Dr. Nicolas Sala, Dr. jur. Rudolf Ménesi, Tamás Schmidt, Dr. Tibor Sahin-Tóth. 


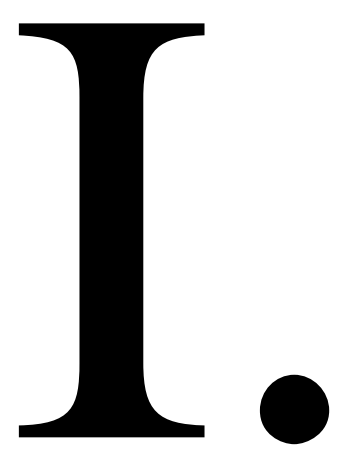




\title{
Myoblast Migration and Directional Persistence Affected by Syndecan-4-Mediated Tiam-1 Expression and Distribution
}

\author{
Daniel Becsky ${ }^{1,+}$, Szuzina Gyulai-Nagy ${ }^{1,+}{ }^{,}$Arpad Balind ${ }^{2}$, Peter Horvath ${ }^{2}$, Laszlo Dux ${ }^{1}$ and \\ Aniko Keller-Pinter ${ }^{1, *}$ \\ 1 Department of Biochemistry, Faculty of Medicine, University of Szeged, H-6720 Szeged, Hungary; \\ becsky.daniel@med.u-szeged.hu (D.B.); szuzina1997@gmail.com (S.G.-N.); \\ dux.laszlo@med.u-szeged.hu (L.D.) \\ 2 Institute of Biochemistry, Biological Research Centre, Hungarian Academy of Sciences, \\ H-6726 Szeged, Hungary; balind.arpad@brc.hu (A.B.); horvath.peter@brc.hu (P.H.) \\ * Correspondence: keller.aniko@med.u-szeged.hu; Tel.: +36-62-545-096 \\ + These authors contributed equally to this work.
}

Received: 19 December 2019; Accepted: 23 January 2020; Published: 27 January 2020

\begin{abstract}
Skeletal muscle is constantly renewed in response to injury, exercise, or muscle diseases. Muscle stem cells, also known as satellite cells, are stimulated by local damage to proliferate extensively and form myoblasts that then migrate, differentiate, and fuse to form muscle fibers. The transmembrane heparan sulfate proteoglycan syndecan-4 plays multiple roles in signal transduction processes, such as regulating the activity of the small GTPase Rac1 (Ras-related C3 botulinum toxin substrate 1) by binding and inhibiting the activity of Tiam1 (T-lymphoma invasion and metastasis-1), a guanine nucleotide exchange factor for Rac1. The Rac1-mediated actin remodeling is required for cell migration. Syndecan-4 knockout mice cannot regenerate injured muscle; however, the detailed underlying mechanism is unknown. Here, we demonstrate that shRNA-mediated knockdown of syndecan-4 decreases the random migration of mouse myoblasts during live-cell microscopy. Treatment with the Rac1 inhibitor NSC23766 did not restore the migration capacity of syndecan-4 silenced cells; in fact, it was further reduced. Syndecan-4 knockdown decreased the directional persistence of migration, abrogated the polarized, asymmetric distribution of Tiam1, and reduced the total Tiam1 level of the cells. Syndecan-4 affects myoblast migration via its role in expression and localization of Tiam1; this finding may facilitate greater understanding of the essential role of syndecan-4 in the development and regeneration of skeletal muscle.
\end{abstract}

Keywords: myoblast; cell migration; Tiam1; syndecan-4; proteoglycan; Rac1; NSC23766; directional persistence; muscle regeneration; skeletal muscle

\section{Introduction}

Skeletal muscle is a highly dynamic tissue that can regenerate successfully following injury, and it can change in size in response to exercise, aging, or diseases (e.g., cancer cachexia, immobilization, muscular dystrophy). The skeletal muscle stem cells, also known as satellite cells, which are localized between the muscle fiber and basal lamina, are responsible for the plasticity, maintenance, and regeneration of skeletal muscle [1,2]. The satellite cells are mitotically and physiologically quiescent in healthy muscle, but they are stimulated by local damage to form myoblasts that subsequently proliferate, migrate, differentiate, and fuse to form multinucleated muscle fibers.

Cell migration is essential for establishing and maintaining the proper organization of multicellular organisms [3]. Cell movement is a complex process that plays an important role in various physiological 
processes including regeneration, wound healing, angiogenesis, embryonic development, and immune cell responses, in addition to tumor progression and metastasis formation. One of the most important conditions for cell movement is the polarization of the cell, in terms of morphology, with the formation of a leading edge at the front and a retracting tail, and the correct positioning of the cellular components [4]. An actin-rich protrusion, called the lamellipodium, must evolve toward migration, thereby creating asymmetry in the cell, with distinct front-rear polarity [5]. Polarization is also defined by the positioning of the cell nucleus and the reorientation of the Golgi network and microtubule organizing center toward the leading edge [6,7]. The Rho family of small GTPases, including Rac1 (Ras-related C3 botulinum toxin substrate 1), Cdc42, and RhoA, plays a fundamental role in the development and maintenance of this front-rear polarity [8].

The Rho GTPases act as molecular switches cycling between an inactive GDP-bound and an active GTP-bound form to bind and activate downstream effector proteins, thereby affecting various signaling pathways [9]. The two-state cycle is regulated by three sets of proteins: the guanine nucleotide exchange factors (GEFs), which catalyze the exchange of GDP for GTP, and GTPase-activating proteins (GAPs), which increase intrinsic GTP hydrolysis, are responsible for Rho GTPases switching between their active and inactive forms, respectively. Moreover, switching between GDP- and GTP-bound states may involve cytosol-membrane translocation, as guanine dissociation inhibitors (GDIs) prevent Rho GTPases from membrane-targeting and activation [10].

Tiam1 (T-lymphoma invasion and metastasis-1) has been identified as a GEF, acting as a specific activator of Rac1 [11], and it plays a key role in pivotal biological processes including cell migration [8] and cell polarization [11]. The Par (partitioning defective) polarity complex, comprising Par3, Par6 and atypical PKC, plays a key role in the development and maintenance of cell polarity [8]. Furthermore, Tiam1, along with the Par polarity complex, stimulates persistent migration by stabilizing the anterior-posterior polarization of migrating cells [12]. Par3 interacts with Tiam1, leading to localized Rac1 activation, creating a gradient of Rac1 and RhoA GTPases in migrating cells: the former is concentrated at the leading edge, and the latter is in the rear of the cell [13]. As Tiam1-mediated Rac1 signaling is required for establishing and maintaining cell polarity [14], impaired Tiam1 signaling inhibits the formation of front-rear polarization in migrating cells thereby inhibiting persistent migration [12].

Syndecan-4 (SDC4) is a ubiquitously expressed transmembrane, heparan sulfate proteoglycan that is a major cell surface marker of satellite cells [15]. Syndecan-4 connects the extracellular matrix to the cytoskeleton, allowing the interaction between the cell and extracellular matrix components, cytokines, or growth factors [16]. The cytoplasmic domain contains one variable and two conserved regions, binding to ezrin, radixin, and moesin proteins, in addition to Src kinase, $\alpha$-actinin, or protein kinase $\mathrm{C} \alpha(\mathrm{PKC} \alpha)$ [17-19], and regulating intracellular $\mathrm{Ca}^{2+}$ concentration [20] or Rac1 activity [21,22]. Syndecan-4 binds and inhibits Tiam1, modulating the activity of Rac1 GTPase [22]. The cytoplasmic domain of syndecan-4 contains a type II PDZ binding site, and Tiam1 exhibit a PDZ domain. Both phosphorylation of the cytoplasmic serine residue of syndecan-4 and the PDZ domain binding of Tiam1 are involved in regulation of the GEF activity of Tiam1, thereby regulating Rac1-GTP level [22].

Syndecan-4 knockout mice cannot regenerate injured skeletal muscle [23], and the precise mechanism underlying this phenomenon is unclear. Given the role of syndecan-4 in the regulation of Rac1 GTPase and the importance of cell migration during muscle regeneration, the aim of our study was to investigate the role of Tiam1-mediated Rac1 activation in syndecan-4-dependent myoblast migration. We have demonstrated that syndecan-4 knockdown decreased migration of myoblasts, accompanied by reduced global Tiam1 expression. Moreover, it abrogated the asymmetric, polarized distribution of Tiam 1 and reduced directional persistence of the movement. 


\section{Results}

\subsection{Syndecan-4 Silencing Reduces Myoblast Migration in a Random Migration Assay}

We verified the effect of syndecan-4 (SDC4) silencing by qPCR technique and Western blotting in C2C12 mouse myoblast cell lines transfected stably with plasmids expressing shRNA specific for syndecan-4 (shSDC4\#1 and shSDC4\#2), and we also tested the effect of the scrambled sequence [24]. The syndecan- 4 expression was significantly decreased in both shSDC4\#1 and shSDC4\#2 cell lines, with the reduction being less in the latter; whereas the scrambled sequence did not affect the syndecan- 4 level [24].

Initially, the effect of syndecan-4 silencing was examined in a random migration assay for $18 \mathrm{~h}$. Representative time-lapse videos are included as Supplementary Materials Videos S1-S4. Syndecan-4 knockdown significantly reduced the total path of migration, both the vectorial distance (i.e. real shift of the cells) and the maximum distance from the origin were decreased; furthermore, the average and maximum speed were also reduced (Figure 1A-E). No significant difference was observed between the non-transfected and scrambled cells.

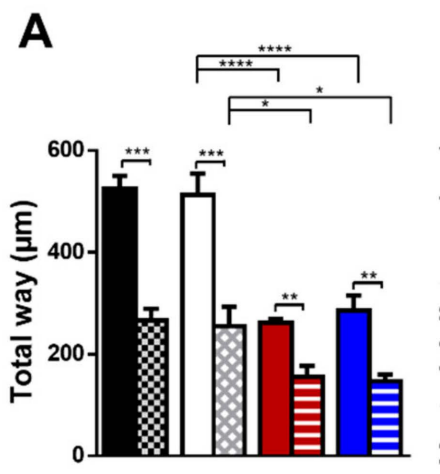

B

C
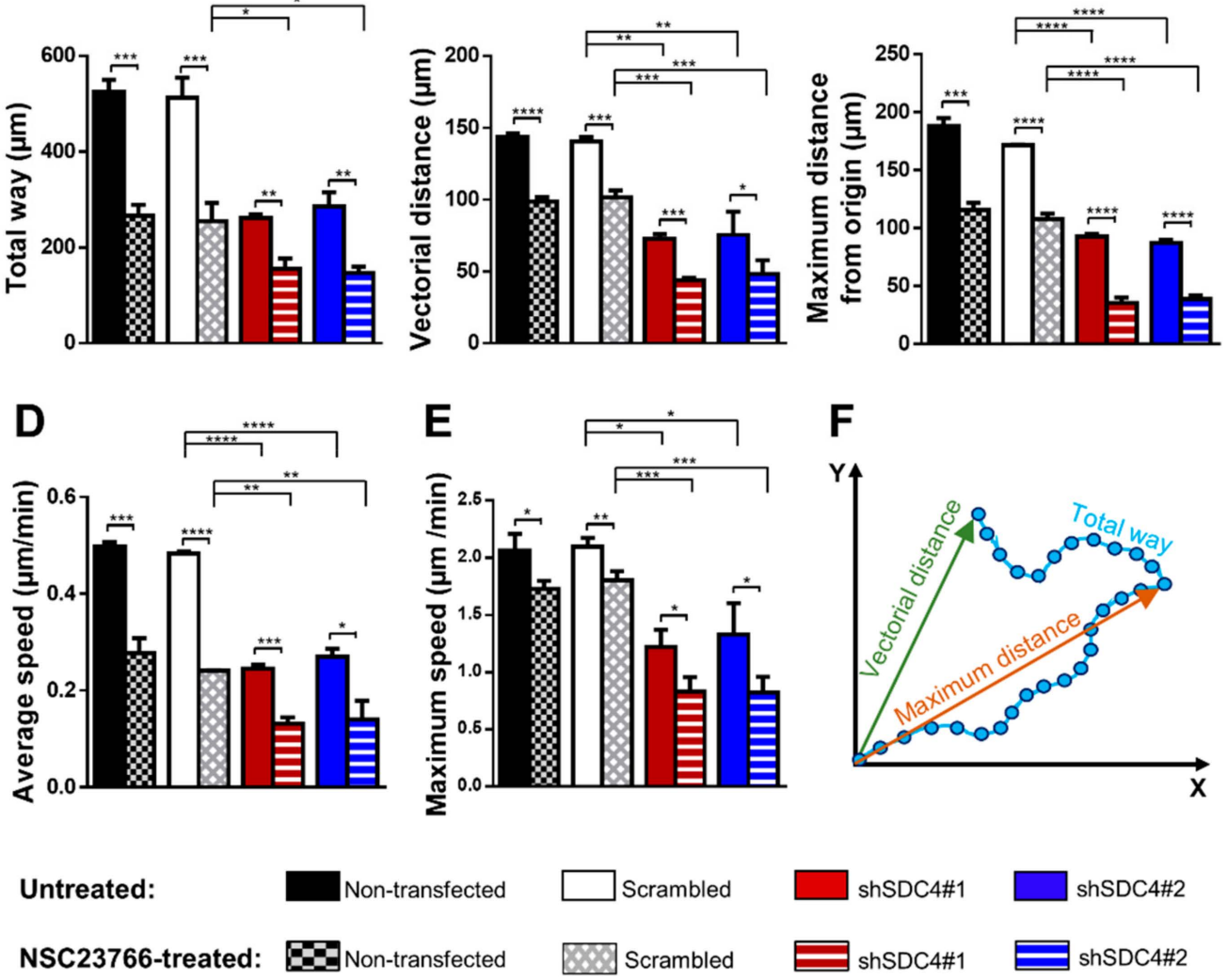

Figure 1. The role of syndecan-4 and Rac1 (Ras-related C3 botulinum toxin substrate 1) in random, two-dimensional migration of myoblasts. The total length of movement (A), the vectorial distance (real displacement of the cells) (B), the maximum distance from the starting point (C), the average cell speed (D), and the maximum speed (E) of the cells are depicted in non-transfected, scrambled, and syndecan-4 silenced (shSDC4\#1, shSDC4\#2) C2C12 myoblast cells either without or with NSC23766 (Rac1 inhibitor) treatment. Schematic representation of the total path, vectorial distance, and maximum distance of the movement (F). The total duration of live cell microscopy: $18 \mathrm{~h}$, frame rate: $3 / 1 \mathrm{~h} ; n=4$ independent experiments; $62-114$ cells/cell line; and 6-8 fields of view/experiment. Data are reported means of the independent experiments + SEM; ${ }^{* * *} p<0.0001 ;{ }^{* *} p<0.001{ }^{* *} p<0.01 ;^{*} p<0.05$. 
Next, we transposed the migratory tracks (total paths) of the individual cells to a common origin to generate the static wind rose plots depicted in Figure 2. The representative wind rose plots depict the migratory tracks of cells based on the position of the $x$ and y coordinates of the movement (Figure 2). The smaller diameters of the wind rose plots in both shSDC4\#1 and shSDC4\#2 lines indicate the reduced motility of these cells (Figure $2 \mathrm{~A}$ ).
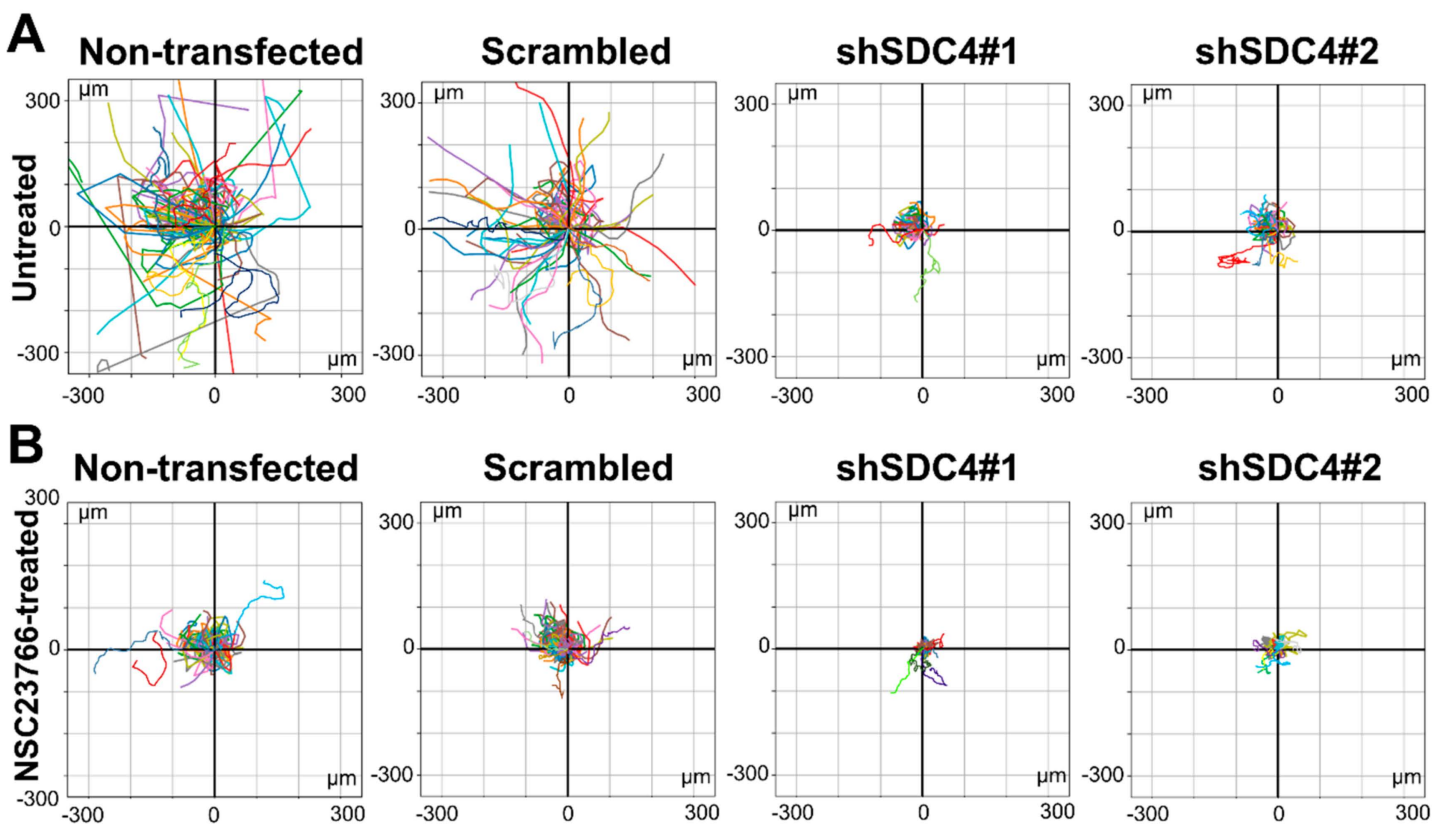

Figure 2. Representative wind-rose plots depict the total path of the cells. The trajectories were shifted to a common origin. Each colored line represents the total path of a single untreated myoblast either without (A) or with NSC23766 treatment (B) in the different cell lines. Total duration of live cell microscopy: $18 \mathrm{~h}$.

\subsection{Inhibition of Rac1 Does Not Restore the Defective Migratory Phenotype of Syndecan-4 Knockdown Cells}

Syndecan-4 knockout causes a steady increase in the levels of activated Rac1-GTPase [21,25-28]. The aim of our next experiment was to study how Rac1 inhibition affects migration, and whether it can improve the decreased migration of syndecan- 4 silenced cells. The activity of Rac1 was specifically inhibited by NSC23766 treatment [29]. Cell migration was examined for $18 \mathrm{~h}$ during a random migration assay. Representative time-lapse videos (Supplementary Materials Videos S5-S8) show the decreased motility of the cells following NSC23766 treatment. During this analysis, the specific inhibition of Rac1 GTPase did not ameliorate the migration defect due to syndecan-4 knockdown. Interestingly, Rac1 inhibition caused further significant reduction in all examined parameters, including the total path of the cells, vectorial and maximum displacement, and average and maximum speed values in all cell lines (Figure 1).

The representative wind rose plots depicting the migratory tracks of the individual cells show the decreased motility of all cell lines upon treatment with the Rac1 inhibitor NSC23766 (Figure 2B). The representative plots clearly show the result of the combined effect of syndecan-4 silencing and Rac1 inhibition. Notably, the migratory parameters of syndecan-4 silenced cell lines further decreased following NSC23766 treatment.

\subsection{Syndecan-4 Knockdown Affects the Directional Persistence of Migration}

The effectiveness of cell migration depends on two essential features: cell-speed and directional persistence. At the cellular level, directional persistence depends on the tenacity of lamellipodial protrusions and the stability of the trailing edge $[27,28]$. To ascertain the stability of the orientation 
of the cell migration, we also calculated the persistence index [29] in the case of the control and the syndecan-4 knockdown cell lines. The values of the persistence index over the timescale represent the time-dependency of the directional persistence showing that these differences can be constantly observed by comparing the cell lines (Figure 3A).
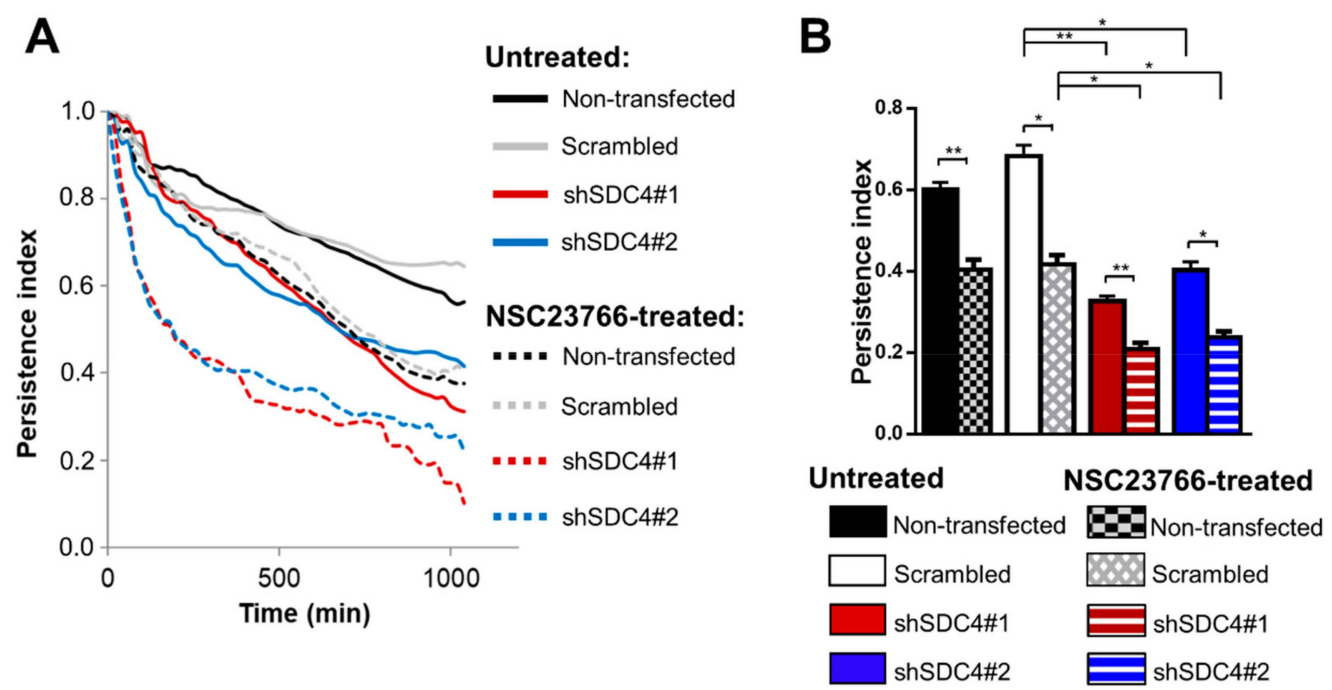

Figure 3. Directional persistence in cell migration. (A) Average persistence index (vectorial distance/total path ratio) over elapsed time indifferent cell lines. (B) Effect of Syndecan-4 (SDC4) silencing and/or Rac1 inhibition (NSC23766 treatment, $50 \mu \mathrm{M}$ ) on persistence index after $18 \mathrm{~h} . n=4$ independent experiments, 62-114 cells/cell line, 6-8 fields of view/experiment, Data are reported as means of the independent experiments + SEM; ${ }^{*} p<0.05 ;{ }^{* *} p<0.01$.

The results show that silencing of syndecan- 4 significantly decreases the persistence index of myoblasts measured after $18 \mathrm{~h}$ movement. There was no significant difference between the non-transfected and scrambled cell lines. NSC23766 treatment of the cells further reduced persistence index in both syndecan-4 knockdown cell lines (Figure 3B). Interestingly, the persistence index of the untreated syndecan-4 knockdown cells was similar to the NSC23766-treated control lines; suggesting that neither high nor low activity of Rac1 favors directional persistence of the migration.

\subsection{Syndecan-4 Affects Tiam1 Expression and Localization}

As the inhibition of Rac1 activity reduced migration ability (in both control and syndecan-4 silenced cell lines), we explored whether we could identify alteration in either distribution or expression of Tiam1. We found an asymmetrical Tiam1 distribution with an increased intensity towards the leading edge in the non-transfected and scrambled cell lines, whereas this peak in the Tiam1-intensity was absent in the syndecan-4 silenced cells (Figure 4A). The representative 2D and 3D pseudocolor images provide better visualization of this altered Tiam1 distribution: the high-intensity red area depicted an asymmetric Tiam 1 enrichment that is mainly adjacent to the nucleus, and homogenous cytoplasmic blue-green intensity was observed in both syndecan-4 silenced cell lines. These quantified results confirm the pervious observations, as the mean intensity values in the individual cells are significantly decreased following syndecan-4 knockdown (Figure 4B), indicative of reduced Tiam1 expression in these cells. To measure the variation in the pixel intensities, the standard deviation of Tiam1 intensity values within each cell were quantified. The standard deviation of intensity values in both syndecan-4 silenced cell lines were significantly lower. The pixel intensity values were closer to the mean intensity, whereas they are spread out over a wider range in the control cells (Figure 4C). 
A
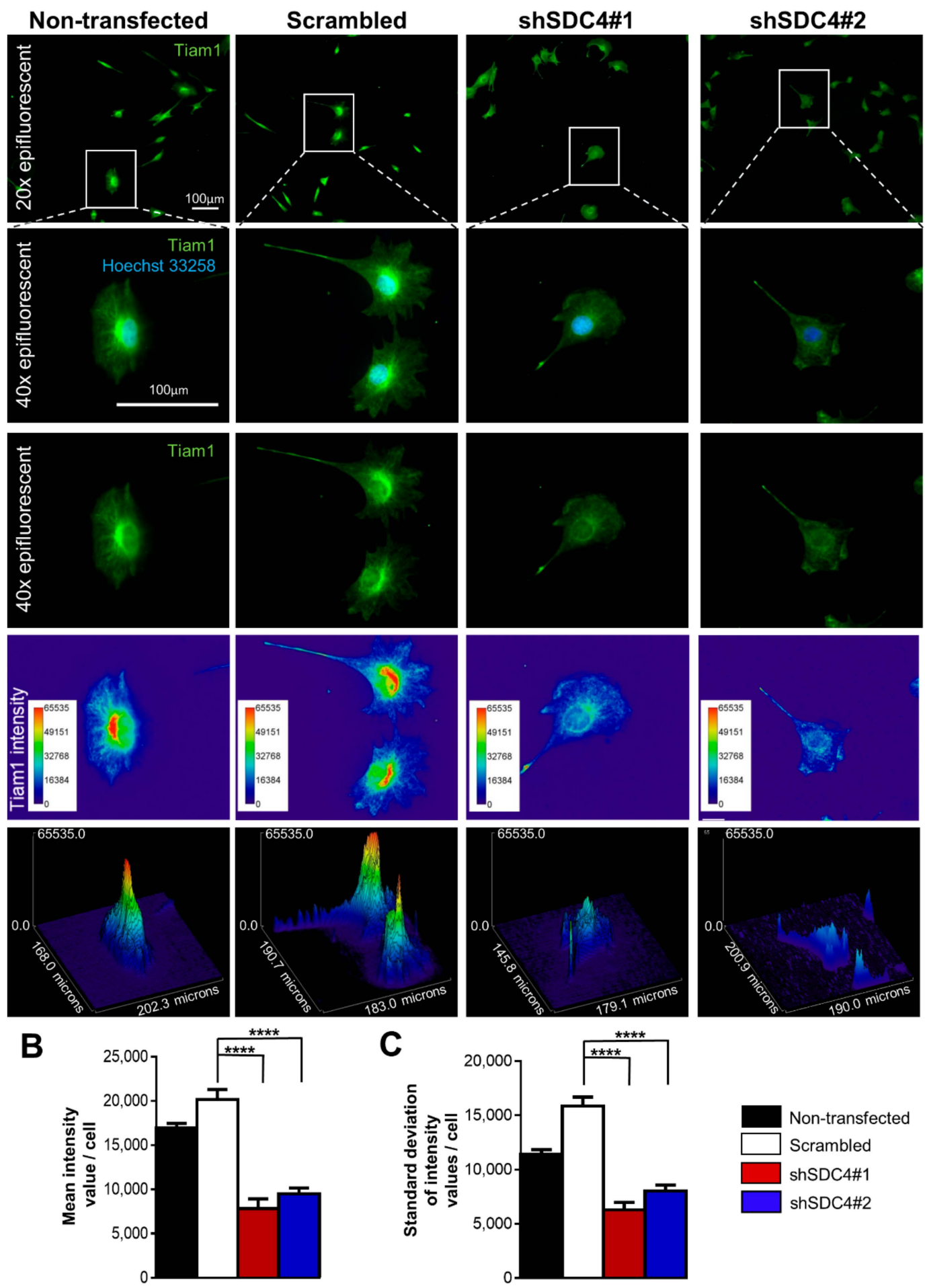

Figure 4. Tiam1 (T-lymphoma invasion and metastasis-1) expression of the different cell lines. (A) Representative images show Tiam1 (green) distribution. The non-transfected and scrambled cells exhibit an asymmetric, polarized Tiam1 arrangement, which is absent due to SDC4 silencing. Nuclei are blue (Hoechst 33258). Representative pseudocolor images (2D and 3D) depict Tiam1 signal intensity. The color was assigned to each pixel based on the pixel intensity value according to the calibration bar (shown on left-hand sides of the images). The mean intensity values in the individual cells are quantified in (B), and the average standard deviation values of the Tiam1 intensities are depicted in (C); $n=4-20$ cells/cell line were analyzed; data are reported as mean+SEM; ${ }^{* * * *} p<0.0001$. 


\section{Discussion}

Tiam1 plays an essential role in pivotal biological processes and has been identified as a nucleotide exchange factor (GEF), a specific activator of the small GTPase Rac1 [11]; however, it is also specific for Cdc42 and to a lesser extent, RhoA [30]. The Par (comprising Par3, Par6, and atypical PKC)-Tiam1 complex stabilizes the front-rear polarization of migratory cells, thereby stimulating persistent and chemotactic migration. Meanwhile, in epithelial cells it controls the establishment of apical-basal polarity, thus showing the control of distinct forms of cellular polarity [12]. Tiam1-mediated Rac1 signaling is vital in establishing and maintaining of cell polarity, and it localizes the Rac1 activity at the leading edge of the migrating cells [8]. Furthermore, impaired Tiam1 signaling was reported to inhibit the formation of front-rear polarization during migration thereby inhibiting persistent migration [12].

The role of syndecan- 4 transmembrane proteoglycan in directional migration, which is essential for several physiological processes, has been identified previously [21,25,31]. Moreover, syndecan-4, by regulating PKC $\alpha$ activity, is responsible for the localization of active Rac1 in membrane protrusions at the leading edge of cells [21], which is necessary for persistent migration. Therefore, knocking-out of syndecan-4 increases the level of delocalized active Rac1 [21]. It is noteworthy that the direct relationship between the syndecan- 4 coordinated persistent migration and the Tiam1-mediated Rac1 activation remains unknown. Earlier, we reported that syndecan-4 binds and inhibits Tiam1, modulating the activity of Rac1 GTPase [24]. Thus, the syndecan-4-Tiam1 complex can regulate the local activity of Rac1.

Both the total amount of Rac1-GTP and its spatial distribution is critical for cell migration. Pankov et al. reported that both very highly activated Rac1 and very low Rac1 activity inhibit migration [32]. Knocking-out of syndecan-4 results in increased Rac1-GTP level [21,25-28], thus syndecan-4 is involved to maintain the low basal activity of Rac1, and here we reported that syndecan-4 silencing decreased the migration of myoblasts. NSC23766 is a cell-permeable specific inhibitor of Rac1 activation by the Rac1-specific GEFs Tiam1 [29]. We hypothesized that Rac1 inhibitor treatment can improve the migratory parameters of syndecan- 4 silenced cells, but the specific inhibition of Rac1 activity by NSC23766 did not rescue the effects of syndecan-4, rather exacerbated it. As NSC23766 treatment does not restore the migratory phenotype of the syndecan- 4 knockdown cells, not only the active Rac1 level but the syndecan-4-dependent localization of Rac1 activity is also important for the correct cell migration.

For the effective cell migration, the establishment of a rear-front Rac1 gradient is essential. The syndecan-4 knockdown resulted in homogenous Tiam1 distribution, which might, in turn cause delocalized Rac1 activation (Figure 5). This can explain the reduced persistence and motility of syndecan-4 knockdown cells (Figure 5). In accordance with our observation, syndecan-4-null fibroblasts migrate randomly as a result of high delocalized Rac1 activity [21]. Interestingly, the polarized distribution of syndecan- 4 was already shown during cytokinesis by its accumulation in the intercellular bridges during cell division [33]. Moreover, the directional persistence index of untreated syndecan-4 knockdown cells was similar to those of the NSC23766-treated non-transfected and scrambled lines; indicating that more than just the absolute amount of active Rac1 is important for the directionality of migration.

In this study, Tiam1 exhibited a strong asymmetrical localization toward the leading edge in non-transfected and scrambled cell lines. The perinuclear accumulation of Tiam1 can also be observed indicating that Tiam1 localizes not only in the leading-edge protrusions but also shows significant accumulation in the Golgi-network in migrating cells. As with other cellular constituents, such as centrosomes, the Golgi-apparatus requires a leader-oriented localization for effective cell migration. The level of Tiam1 expression seems to be correlated with cell motility [34]. In our study, we detected a significantly higher signal intensity of Tiam1 in non-transfected and scrambled cell lines, than in the syndecan-4 silenced cells (Figure 4A). These findings may indicate increased expression of Tiam1 in control cell lines. Syndecan-4 silencing reduces Tiam1 signal intensity and abrogates the strong, asymmetrical Tiam1 gradient towards the leading edge. 

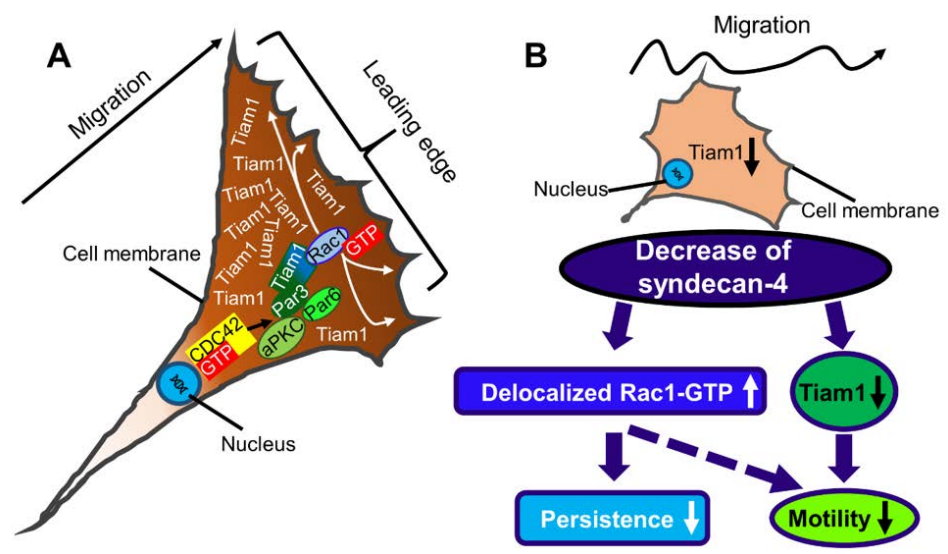

Figure 5. (A) Schematic representation of a migrating cell with a characteristic shape. The direction of migration is well defined, and the Tiam1 is asymmetrically accumulated at the leading edge resulting in Rac1 activation and lamellipodia formation. (B) Knocking-down of syndecan-4 resulted in decreased Tiam1 expression, abrogated the asymmetrical distribution of Tiam1, decreased the directional persistence of the movement, and decreased cell motility. Par3, Par6: Partitioning defective 3,6; aPKC: atypical PKC; Tiam1: T-lymphoma invasion and metastasis-1; $\uparrow$ : increase; $\downarrow$ : decrease.

Given the role of Tiam 1 and Rac1 in cell migration, several studies have explored the prognostic value of Tiam1 in patients with solid tumors. High Tiam1 expression has been significantly associated with shorter survival and positive lymphatic metastasis in patients with malignant solid tumors, and Tiam1 may present a promising prognostic biomarker and an effective therapeutic target for treating malignancies [35]. For example, a study has shown that Tiam1 expression correlated with cell motility in human breast cancer cells and is required to support the motile phenotype [34]. The authors reported the localization of endogenous Tiam1 to the Golgi, and demonstrated the role of this in Golgi reorientation, suggesting that it may support motility through a mechanism that is discrete from its known function in leading-edge dynamics [34]. Similarly, we identified accumulation of Tiam 1 in intracellular compartments. In accordance with these Tiam 1 expression data, the syndecan-4 knockdown cells in our model system exhibited both reduced expression of Tiam1 and reduced motility.

Overall, our results indicate a critical role of syndecan- 4 during myoblast migration, which may contribute to an exploration of the essential role of syndecan- 4 in the development and regeneration of skeletal muscle. We hope that our findings will facilitate understanding of the mechanisms underlying myoblast migration during embryonic development and postnatal muscle regeneration.

\section{Materials and Methods}

\subsection{Cell Culture and Plasmids}

C2C12 mouse myoblast (ATCC; Manassas, VA, USA) cultures were maintained in medium containing 80\% high-glucose Dulbecco's modified Eagle's medium $(4.5 \mathrm{~g} / \mathrm{L}$ glucose containing 584 $\mathrm{mg} / \mathrm{L}$ glutamine and $110 \mathrm{mg} / \mathrm{L}$ pyruvate; Corning, New York, NY, USA), 20\% fetal bovine serum (Gibco/Thermo Fisher Scientific, Waltham, MA, USA), and $65 \mu \mathrm{g} / \mathrm{mL}$ gentamicin (Lonza, Basel, Switzerland). Cells were transfected stably using shRNA (short hairpin RNA) expressing plasmids (OriGene, TR513122; Rockville, MD, USA) and X-tremeGENE transfection reagent (Roche, Basel, Switzerland). For syndecan-4 knockdown, we applied plasmids targeting the following sequences: 5'-GAA CTG GAA GAG AAT GAG GTC ATT CCT AA-3' (shSDC4\#1) or 5'-GCG GCG TGG TAG GCA TCC TCT TTG CCG TT-3' (shSDC4\#2). The scrambled plasmid targeted the $5^{\prime}$-GCA CTA CCA GAG CTA ACT CAG ATA GTA CT-3' sequence. Selection of the transfected cells was obtained by adding $4 \mu \mathrm{g} / \mathrm{mL}$ puromycin (Sigma-Aldrich, St. Louis, MO, USA) to the culturing medium. 


\subsection{Time-Lapse Imaging of Live Cells}

Live-cell imaging was carried out using the Operetta high-content imaging system with a $20 \times$ objective (PerkinElmer, Inc., Waltham, MA, USA). For imaging, cells were seeded in 24-well plates at a density of $7.5 \times 10^{3}$ cells/well. After $60 \mathrm{~min}$, the medium was changed to a serum-reduced one to suppress cell division, and $24 \mathrm{~h}$ later the nuclei were stained with Hoechst 33342 (1:2000, $1 \mathrm{mg} / \mathrm{mL}$ stock solution; Sigma-Aldrich), and Rac1 was inhibited by NSC23766 treatment during the measurement (50 $\mu \mathrm{M}$; Tocris Bioscience, Bristol, United Kingdom). Time-lapse images were obtained automatically at $20 \mathrm{~min}$ intervals for $18 \mathrm{~h}$ at $37^{\circ} \mathrm{C}$ and $5 \% \mathrm{CO}_{2}$.

\subsection{Single-Cell Tracking of Cultured Myoblasts}

Time-lapse microscopic images were analyzed using ImageJ (National Institutes of Health, Bethesda, MD, USA, https://imagej.nih.gov/ij/) and CellTracker (http://celltracker.website/) software programs. The nuclei were tracked manually through every frame, and the $x$ and $y$ coordinates of the movement were recorded. We excluded dying, dividing, or damaged cells from the analysis. Based on the nuclear coordinates, the length of total path, the vectorial distance (i.e., real shift of the cell), and maximal distance from origin, the average and maximal speed were calculated. The directionality of the cell movement was described by the persistence index, which was defined by the ratio of the vectorial distance (the distance between the origin and the endpoint of the movement) and the length of total path.

\subsection{Trajectories and Wind-Rose Diagrams}

To illustrate the trajectories, the migratory tracks of the individual cells were transposed to a common origin using Excel DiPer Plot_At_Origin macro [36]. The wind rose plots created draw individual cell paths based on the $x$ and $y$ coordinates of the cell tracking data.

\subsection{Fluorescent Staining and Microscopy}

For fluorescent staining, cells were seeded for $24 \mathrm{~h}$ onto fetal bovine serum (Gibco/Thermo Fisher Scientific, Waltham, MA, USA) coated glass coverslips (Hirschmann Laborgeräte GmbH \& Co. KG, Eberstadt, Germany), fixed with $4 \%$ paraformaldehyde (Molar Chemicals Kft., Halásztelek, Hungary) for $10 \mathrm{~min}$ at room temperature, permeabilized with 0.3\% Tween 20 (Sigma-Aldrich, Inc., St. Louis, Missouri, USA), and blocked with 1\% bovine serum albumin (Sigma-Aldrich) in PBS. Rabbit polyclonal anti-Tiam1 primary antibody (OST00085W; Invitrogen, Carlsbad, CA, USA) was visualized with the appropriate Alexa488-conjugated secondary antibody (Invitrogen). The nuclei were counterstained with Hoechst 33258 (0.01 mg/mL, Sigma-Aldrich). Epi-fluorescence images were obtained on a Nikon Eclipse Ti-E microscope (Nikon Instruments Inc., 1300 Walt Whitman Road Melville, NY 11747-3064, USA) with $40 \times$ objectives.

\subsection{Evaluation of Tiam1 Immunostaining}

The epifluorescent images were recolored as heat maps using ImageJ (National Institutes of Health, Bethesda, MD, USA, https://imagej.nih.gov/ij/) image analysis program (Image>Lookup Tables). Each pixel was colored based on the pixel intensity value (16-bit images; 0-65535 gray value) according to the scale bar shown in the images (the lowest intensity pixels are shown as blue; the highest intensity pixels are shown as red). The contours of the individual cells were drawn (Analyze $>$ Analyze particles), and the average pixel intensity within the border of the cells were quantified (Analyze>Measure) in the different cell lines. The intensity value of each pixel was measured within the selected area and the sum of the intensities was divided by the area of the cell to obtain the average Tiam 1 intensity value of the individual cells. To measure the amount of variation in the pixel intensities, the standard deviation of the intensity values was also calculated in every cell (Analyze>Measure). 


\subsection{Statistical Analysis}

Differences between groups were analyzed using a one-way ANOVA, followed by the Scheffe post-hoc test. GraphPad Prism 7.0 (GraphPad Software Inc., San Diego, CA, USA) was used for graphing and statistical analyses. The data are expressed as means + standard errors of the means. A $p$ value $<0.05$ was considered as significantly different.

Supplementary Materials: Supplementary materials can be found at http://www.mdpi.com/1422-0067/21/3/823/s1.

Author Contributions: Conceptualization, A.K.-P.; data curation, D.B., S.G.-N., A.B., A.K.-P.; writing-original draft preparation, B.D., S.G.-N., A.K.-P.; writing-review and editing, A.K.-P., L.D.; visualization, D.B., S.G.-N.; supervision, A.K.-P., P.H., L.D.; funding acquisition, A.K.-P., L.D., P.H. All authors have read and agreed to the published version of the manuscript.

Funding: This research was supported by the National Research, Development and Innovation Office of Hungary (grant numbers: GINOP-2.3.2-15-2016-00040 (MYOTeam), EFOP-3.6.2-16-2017-00006, NKFI K 132446). The work was further supported by the János Bolyai Research Scholarship of the Hungarian Academy of Sciences (to A.K.-P.), UNKP-19-4-SZTE-23 New National Excellence Program of the Ministry for Innovation and Technology Sciences (to A.K.-P.), and was conducted with support from the Szeged Scientists Academy under the sponsorship of the Hungarian Ministry of Human Capacities (grant number EMMI:13725-2/2018/INTFIN, to S.G.-N.). P.H. acknowledge support from the LENDULET-BIOMAG Grant (grant number 2018-342) and the European Regional Development Funds (grant numbers: GINOP-2.3.2-15-2016-00006, GINOP-2.3.2-15-2016-00026, and GINOP-2.3.2-15-2016-00037).

Acknowledgments: The authors thank Zita Makrane Felho and Laszlone Csontos (University of Szeged) for their excellent technical assistance.

Conflicts of Interest: The authors declare no conflict of interest.

\section{Abbreviations}

GDI Guanine dissociation inhibitors

GEF Guanine nucleotide exchange factor

GAP GTPase activating protein

Par Partitioning defective

PKC $\alpha \quad$ Protein kinase $\mathrm{C} \alpha$

Rac1 Ras-related C3 botulinum toxin substrate 1

SDC4 Syndecan-4

Tiam1 T-lymphoma invasion and metastasis-1

\section{References}

1. Mauro, A.; Adams, W.R. The structure of the sarcolemma of the frog skeletal muscle fiber. J. Biophys. Biochem. Cytol. 1961, 10, 177-185. [CrossRef]

2. Chang, N.C.; Rudnicki, M.A. Satellite cells: The architects of skeletal muscle. Curr. Top. Dev. Biol. 2014, 107, 161-181. [CrossRef]

3. Trepat, X.; Chen, Z.; Jacobson, K. Cell migration. Compr. Physiol. 2012, 2, 2369-2392. [CrossRef] [PubMed]

4. Ridley, A.J.; Schwartz, M.A.; Burridge, K.; Firtel, R.A.; Ginsberg, M.H.; Borisy, G.; Parsons, J.T.; Horwitz, A.R. Cell migration: integrating signals from front to back. Sci. (New York N.Y.) 2003, 302, 1704-1709. [CrossRef] [PubMed]

5. Krause, M.; Gautreau, A. Steering cell migration: Lamellipodium dynamics and the regulation of directional persistence. Nat. Rev. Mol. Cell Biol. 2014, 15, 577-590. [CrossRef] [PubMed]

6. Vicente-Manzanares, M.; Webb, D.J.; Horwitz, A.R. Cell migration at a glance. J. Cell Sci. 2005, 118, 4917-4919. [CrossRef]

7. Mardakheh, F.K.; Self, A.; Marshall, C.J. RHO binding to FAM65A regulates Golgi reorientation during cell migration. J. Cell Sci. 2016, 129, 4466-4479. [CrossRef]

8. Iden, S.; Collard, J.G. Crosstalk between small GTPases and polarity proteins in cell polarization. Nat. Rev. Mol. Cell Biol. 2008, 9, 846-859. [CrossRef]

9. Hall, A. Rho family GTPases. Biochem. Soc. Trans. 2012, 40, 1378-1382. [CrossRef] 
10. Hodge, R.G.; Ridley, A.J. Regulating Rho GTPases and their regulators. Nat. Rev. Mol. Cell Biol. 2016, 17, 496-510. [CrossRef]

11. Mertens, A.E.; Pegtel, D.M.; Collard, J.G. Tiam1 takes PARt in cell polarity. Trends Cell Biol. 2006, 16, 308-316. [CrossRef]

12. Pegtel, D.M.; Ellenbroek, S.I.; Mertens, A.E.; van der Kammen, R.A.; de Rooij, J.; Collard, J.G. The Par-Tiam1 complex controls persistent migration by stabilizing microtubule-dependent front-rear polarity. Curr. Biol. Cb, 2007; 17, 1632-1634. [CrossRef]

13. Wang, S.; Watanabe, T.; Matsuzawa, K.; Katsumi, A.; Kakeno, M.; Matsui, T.; Ye, F.; Sato, K.; Murase, K.; Sugiyama, I.; et al. Tiam1 interaction with the PAR complex promotes talin-mediated Rac1 activation during polarized cell migration. J. Cell Biol. 2012, 199, 331-345. [CrossRef] [PubMed]

14. Pasten, C.; Cerda, J.; Jausoro, I.; Court, F.A.; Caceres, A.; Marzolo, M.P. ApoER2 and Reelin are expressed in regenerating peripheral nerve and regulate Schwann cell migration by activating the Rac1 GEF protein, Tiam1. Mol. Cell. Neurosci. 2015, 69, 1-11. [CrossRef] [PubMed]

15. Cornelison, D.D.; Filla, M.S.; Stanley, H.M.; Rapraeger, A.C.; Olwin, B.B. Syndecan-3 and syndecan-4 specifically mark skeletal muscle satellite cells and are implicated in satellite cell maintenance and muscle regeneration. Dev. Biol. 2001, 239, 79-94. [CrossRef]

16. Elfenbein, A.; Simons, M. Syndecan-4 signaling at a glance. J. Cell Sci. 2013, 126, 3799-3804. [CrossRef] [PubMed]

17. Koo, B.K.; Jung, Y.S.; Shin, J.; Han, I.; Mortier, E.; Zimmermann, P.; Whiteford, J.R.; Couchman, J.R.; Oh, E.S.; Lee, W. Structural basis of syndecan-4 phosphorylation as a molecular switch to regulate signaling. J. Mol. Biol. 2006, 355, 651-663. [CrossRef] [PubMed]

18. Greene, D.K.; Tumova, S.; Couchman, J.R.; Woods, A. Syndecan-4 associates with alpha-actinin. J. Biol. Chem. 2003, 278, 7617-7623. [CrossRef] [PubMed]

19. Multhaupt, H.A.; Yoneda, A.; Whiteford, J.R.; Oh, E.S.; Lee, W.; Couchman, J.R. Syndecan signaling: when, where and why? J. Physiol. Pharmacol. Off. J. Pol. Physiol. Soc. 2009, 60 Suppl 4, 31-38.

20. Gopal, S.; Sogaard, P.; Multhaupt, H.A.; Pataki, C.; Okina, E.; Xian, X.; Pedersen, M.E.; Stevens, T.; Griesbeck, O.; Park, P.W.; et al. Transmembrane proteoglycans control stretch-activated channels to set cytosolic calcium levels. J. Cell Biol. 2015, 210, 1199-1211. [CrossRef]

21. Bass, M.D.; Roach, K.A.; Morgan, M.R.; Mostafavi-Pour, Z.; Schoen, T.; Muramatsu, T.; Mayer, U.; Ballestrem, C.; Spatz, J.P.; Humphries, M.J. Syndecan-4-dependent Rac1 regulation determines directional migration in response to the extracellular matrix. J. Cell Biol. 2007, 177, 527-538. [CrossRef]

22. Keller-Pinter, A.; Ughy, B.; Domoki, M.; Pettko-Szandtner, A.; Letoha, T.; Tovari, J.; Timar, J.; Szilak, L. The phosphomimetic mutation of syndecan-4 binds and inhibits Tiam1 modulating Rac1 activity in PDZ interaction-dependent manner. PLoS ONE 2017, 12, e0187094. [CrossRef] [PubMed]

23. Cornelison, D.D.; Wilcox-Adelman, S.A.; Goetinck, P.F.; Rauvala, H.; Rapraeger, A.C.; Olwin, B.B. Essential and separable roles for Syndecan-3 and Syndecan-4 in skeletal muscle development and regeneration. Genes Dev. 2004, 18, 2231-2236. [CrossRef] [PubMed]

24. Keller-Pinter, A.; Szabo, K.; Kocsis, T.; Deak, F.; Ocsovszki, I.; Zvara, A.; Puskas, L.; Szilak, L.; Dux, L. Syndecan-4 influences mammalian myoblast proliferation by modulating myostatin signalling and G1/S transition. Febs Lett. 2018, 592, 3139-3151. [CrossRef] [PubMed]

25. Brooks, R.; Williamson, R.; Bass, M. Syndecan-4 independently regulates multiple small GTPases to promote fibroblast migration during wound healing. Small Gtpases 2012, 3, 73-79. [CrossRef]

26. Saoncella, S.; Calautti, E.; Neveu, W.; Goetinck, P.F. Syndecan-4 regulates ATF-2 transcriptional activity in a Rac1-dependent manner. J. Biol. Chem. 2004, 279, 47172-47176. [CrossRef] [PubMed]

27. Tkachenko, E.; Elfenbein, A.; Tirziu, D.; Simons, M. Syndecan-4 clustering induces cell migration in a PDZ-dependent manner. Circ. Res. 2006, 98, 1398-1404. [CrossRef]

28. Matthews, H.K.; Marchant, L.; Carmona-Fontaine, C.; Kuriyama, S.; Larrain, J.; Holt, M.R.; Parsons, M.; Mayor, R. Directional migration of neural crest cells in vivo is regulated by Syndecan-4/Rac1 and non-canonical Wnt signaling/RhoA. Dev. (Camb. Engl.) 2008, 135, 1771-1780. [CrossRef] [PubMed]

29. Gao, Y.; Dickerson, J.B.; Guo, F.; Zheng, J.; Zheng, Y. Rational design and characterization of a Rac GTPase-specific small molecule inhibitor. Proc. Natl. Acad. Sci. USA 2004, 101, 7618-7623. [CrossRef]

30. Boissier, P.; Huynh-Do, U. The guanine nucleotide exchange factor Tiam1: a Janus-faced molecule in cellular signaling. Cell. Signal. 2014, 26, 483-491. [CrossRef] 
31. Carmona-Fontaine, C.; Matthews, H.; Mayor, R. Directional cell migration in vivo: Wnt at the crest. Cell Adhes. Migr. 2008, 2, 240-242. [CrossRef]

32. Pankov, R.; Endo, Y.; Even-Ram, S.; Araki, M.; Clark, K.; Cukierman, E.; Matsumoto, K.; Yamada, K.M. A Rac switch regulates random versus directionally persistent cell migration. J. Cell Biol. 2005, 170, 793-802. [CrossRef] [PubMed]

33. Keller-Pinter, A.; Bottka, S.; Timar, J.; Kulka, J.; Katona, R.; Dux, L.; Deak, F.; Szilak, L. Syndecan-4 promotes cytokinesis in a phosphorylation-dependent manner. Cell Mol. Life Sci. 2010, 67, 1881-1894. [CrossRef] [PubMed]

34. Adams, H.C.; Chen, R.; Liu, Z.; Whitehead, I.P. Regulation of breast cancer cell motility by T-cell lymphoma invasion and metastasis-inducing protein. Breast Cancer Res. Bcr 2010, 12, R69. [CrossRef] [PubMed]

35. Ding, J.; Yang, F.; Wu, W. Tiam1 high expression is associated with poor prognosis in solid cancers: A meta-analysis. Medicine 2019, 98, e17529. [CrossRef] [PubMed]

36. Gorelik, R.; Gautreau, A. Quantitative and unbiased analysis of directional persistence in cell migration. Nat. Protoc. 2014, 9, 1931-1943. [CrossRef]

(C) 2020 by the authors. Licensee MDPI, Basel, Switzerland. This article is an open access article distributed under the terms and conditions of the Creative Commons Attribution (CC BY) license (http://creativecommons.org/licenses/by/4.0/). 


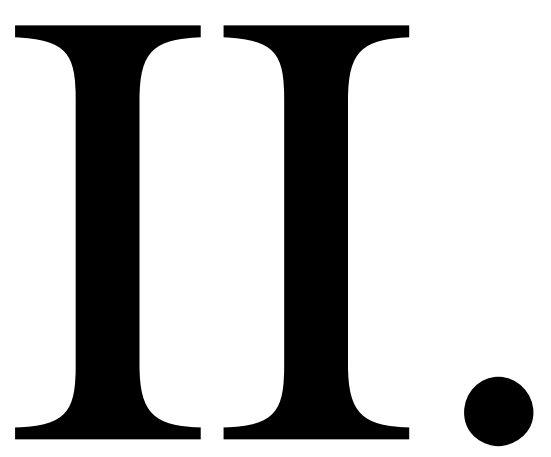




\section{Syndecan-4 Modulates Cell Polarity and Migration by Influencing Centrosome Positioning and Intracellular Calcium Distribution}

\begin{abstract}
Daniel Becsky ${ }^{1 \dagger}$, Kitti Szabo ${ }^{1+}$, Szuzina Gyulai-Nagy ${ }^{1}$, Tamas Gajdos², Zsuzsa Bartos ${ }^{3}$, Arpad Balind ${ }^{4}$, Laszlo Dux ${ }^{1}$, Peter Horvath ${ }^{4}$, Miklos Erdelyi², Laszlo Homolya ${ }^{3}$ and Aniko Keller-Pinter ${ }^{\text {* }}$

'Department of Biochemistry, Faculty of Medicine, University of Szeged, Szeged, Hungary, ${ }^{2}$ Department of Optics and Quantum Electronics, Faculty of Science and Informatics, University of Szeged, Szeged, Hungary, ${ }^{3}$ Institute of Enzymology, Research Centre for Natural Sciences, Hungarian Academy of Sciences Center of Excellence, Budapest, Hungary, ${ }^{4}$ Institute of Biochemistry, Biological Research Centre, Hungarian Academy of Sciences, Szeged, Hungary
\end{abstract}

Efficient cell migration requires cellular polarization, which is characterized by the formation of leading and trailing edges, appropriate positioning of the nucleus and reorientation of the Golgi apparatus and centrosomes toward the leading edge. Migration also requires the development of an asymmetrical front-to-rear calcium $\left(\mathrm{Ca}^{2+}\right)$ gradient to regulate focal adhesion assembly and actomyosin contractility. Here we demonstrate that silencing of syndecan-4, a transmembrane heparan sulfate proteoglycan, interferes with the correct polarization of migrating mammalian myoblasts (i.e., activated satellite stem cells). In particular, syndecan-4 knockdown completely abolished the intracellular $\mathrm{Ca}^{2+}$ gradient, abrogated centrosome reorientation and thus decreased cell motility, demonstrating the role of syndecan-4 in cell polarity. Additionally, syndecan-4 exhibited a polarized distribution during migration. Syndecan-4 knockdown cells exhibited decreases in the total movement distance during directional migration, maximum and vectorial distances from the starting point, as well as average and maximum cell speeds. Super-resolution direct stochastic optical reconstruction microscopy images of syndecan-4 knockdown cells revealed nanoscale changes in the actin cytoskeletal architecture, such as decreases in the numbers of branches and individual branch lengths in the lamellipodia of the migrating cells. Given the crucial importance of myoblast migration during embryonic development and postnatal muscle regeneration, we conclude that our results could facilitate an understanding of these processes and the general role of syndecan-4 during cell migration.

Received: 22 June 2020 Accepted: 17 August 2020 Published: 15 October 2020

Citation:

Becsky D, Szabo K Gyulai-Nagy S, Gajdos T, Bartos Z, Balind A, Dux L, Horvath P, Erdelyi M, Homolya L and Keller-Pinter A (2020) Syndecan-4 Modulates Cell Polarity and Migration by Influencing Centrosome Positioning and Intracellular Calcium Distribution. Front. Cell Dev. Biol. 8:575227. doi: 10.3389/fcell.2020.575227 Keywords: syndecan-4, proteoglycan, cell polarity, super-resolution microscopy, actin, calcium, centrosome, cell
migration

\section{INTRODUCTION}

Cell migration is a fundamentally important factor in various physiological and pathological processes, including morphogenesis, immune surveillance, tissue regeneration, and cancer cell metastasis (Ridley et al., 2003). Cell motility and directed migration require the establishment of cell polarization, defined as the formation of distinct front and rear cellular areas. This process is characterized by the emergence of an actin-mediated lamellipodial membrane protrusion, which 
forms the leading edge, as well as the development of a retracting tail. The leading edge protrusions depend on polarized intracellular signaling processes. Polarization is also defined by the positioning of the cell nucleus and reorientation of the Golgi network and microtubule organizing center toward the leading edge (Vicente-Manzanares et al., 2005; Zhang and Wang, 2017). Cell motility is orchestrated by the formation of integrin-dependent adhesions to the surrounding matrix and the detachment of these adhesions from distinct regions at the rear of the cell (Lauffenburger and Horwitz, 1996; Ridley et al., 2003). These mechanisms direct the cell motility cycle and are required for cell migration in response to various factors. However, the mechanism by which this motility system integrates extracellular signals with cell polarity and cytoskeletal remodeling to promote directionally persistent migration remains unclear.

Calcium $\left(\mathrm{Ca}^{2+}\right)$ has been identified as an essential factor in cell migration. $\mathrm{Ca}^{2+}$ forms an increasing front-rear gradient that is involved in the disassembly of focal adhesions and, consequently, the rear-end retraction and the movement of the cell. This essential front-rear polarity is maintained by restricting the spontaneous formation of lamellipodia at the trailing edges of migrating cells (Tsai et al., 2015; Kim et al., 2016). The steering of membrane protrusions is directed by a localized $\mathrm{Ca}^{2+}$ influx created by stretch-activated $\mathrm{Ca}^{2+}$ channels in the front of a migrating cell, whereas other types of $\mathrm{Ca}^{2+}$ influx have been reported to mediate the detachment of rear protrusions (Kim et al., 2016). However, previous reports describing the coordination of cell migration by the $\mathrm{Ca}^{2+}$ gradient have provided limited insights into cell motility and the formation of these gradients.

Syndecans are a family of four transmembrane proteoglycans, each of which comprises a variable $\mathrm{N}$-terminal ectodomain, a highly conserved short transmembrane and a C-terminal cytoplasmic domains (Zimmermann and David, 1999). Three syndecans are distributed in a tissue-specific manner (Xian et al., 2010; Elfenbein and Simons, 2013): syndecan-1 is mainly present in epithelial cells, syndecan-2 is expressed in mesenchymal cell types and developing neural tissues, whereas syndecan-3 is present in neural tissues and the developing musculoskeletal system. In contrast, syndecan-4 is expressed ubiquitously (Xian et al., 2010). Usually, the ectodomains of syndecans contain three heparan sulfate chains attached to a serine residue via tetrasaccharide linkers (Carey, 1997), although syndecan-1 and syndecan-3 possess additional chondroitin sulfate chains. The interactions of the ectodomain with extracellular matrix molecules, fibronectin, matrix metalloproteinases, growth factors and other cell surface receptors (e.g., integrins) activate downstream signaling pathways. The cytoplasmic domain comprises a variable region unique to each member of the syndecan family, as well as two conserved regions that interact with four-point-one, ezrin, radixin, and moesin (FERM) proteins; Src kinase; and cortactin (Granes et al., 2003). In syndecan-4, the variable region binds and activates the catalytic domain of protein kinase $\mathrm{C} \alpha(\mathrm{PKC} \alpha)$ (Koo et al., 2006), as well as directly binds $\alpha$-actinin in a beta-integrin-independent manner (Greene et al., 2003). The ability of syndecan- 4 to link the extracellular matrix and cytoskeleton enables this proteoglycan to contribute to several outside-in and inside-out signaling events, such as the sequestration and concentration of matrix components, as well as effects on cell-matrix adhesion, endocytosis, exosome biogenesis or cytokinesis (Keller-Pinter et al., 2010; Elfenbein and Simons, 2013; Afratis et al., 2017). Syndecan-4 also regulates the activity of the small GTPase Rac1 (Bass et al., 2007; Keller-Pinter et al., 2017) and the level of intracellular $\mathrm{Ca}^{2+}$ (Liu et al., 2012; Gopal et al., 2015), and contributes to the phosphorylation of focal adhesion kinase (FAK) (Wilcox-Adelman et al., 2002).

Syndecans play an important role in tissue regeneration (Chung et al., 2016). For example, the skeletal muscle is renewed constantly in response to injury, exercise or muscle diseases. During the repair process, activated stem (i.e., satellite) cells form myoblasts that proliferate, migrate to the injured site, differentiate and fuse into polynuclear myotubes (Schultz and McCormick, 1994; Hawke and Garry, 2001). Syndecan4 is a cell surface marker of both quiescent and proliferating satellite cells (Cornelison et al., 2001). Although syndecan4 knockout mice cannot regenerate damaged muscle tissue (Cornelison et al., 2004), the details of the underlying mechanism remain unknown. Previously, we reported that syndecan-4 affects myoblast proliferation by modulating myostatin signaling and the G1/S transition in cell cycle (Keller-Pinter et al., 2018), and directional persistence of random cell migration is affected by syndecan-4-mediated Tiam-1 expression and distribution (Becsky et al., 2020). In this study, we demonstrated that syndecan-4 knockdown induced nanoscale alterations in the lamellipodial actin fiber structure of migrating myoblasts. Moreover, we found that syndecan-4 distributes asymmetrically during cell migration and determines cellular polarity by influencing the positioning of centrosomes and the development of the front-rear $\mathrm{Ca}^{2+}$ gradient. Although several previous reports have described a role for syndecan- 4 in cell migration, here we present a super-resolution structure of the actin cytoskeleton. Moreover, this is the first report to describe the role of syndecan- 4 in the development of the $\mathrm{Ca}^{2+}$ gradient and centrosome positioning in a migrating cell.

\section{MATERIALS AND METHODS}

\section{Cell Culture and Plasmids}

C2C12 mouse myoblast cells (ATCC; Manassas, VA, United States) were cultured in high-glucose Dulbecco's modified Eagle's medium containing $4.5 \mathrm{~g} / \mathrm{L}$ glucose, $584 \mathrm{mg} / \mathrm{L}$ glutamine and $110 \mathrm{mg} / \mathrm{L}$ pyruvate (Corning, NY, United States) supplemented with $65 \mu \mathrm{g} / \mathrm{mL}$ gentamicin (Lonza, Basel, Switzerland), and $20 \%$ fetal bovine serum (Gibco/Thermo Fisher Scientific, Waltham, MA, United States). To achieve syndecan-4 knockdown, C2C12 cells were transfected stably with plasmids expressing short hairpin RNAs (shRNAs) specific for mouse syndecan-4 (shSDC4\#1 and shSDC4\#2) or a scrambled target sequence. The plasmids were obtained from OriGene (TR513122; Rockville, MD, United States) and targeted the following sequences: 5'-GAA CTG GAA GAG AAT GAG GTC ATT CCT AA-3' (shSDC4\#1), 5'-GCG GCG TGG TAG GCA TCC TCT TTG CCG TT-3' (shSDC4\#2) and 5'-GCA CTA 
CCA GAG CTA ACT CAG ATA GTA CT-3' (scrambled). $\mathrm{X}$-tremeGENE transfection reagent (Roche, Basel, Switzerland) was used for the transfection procedures. Transfected cells were then selected in medium containing $4 \mu \mathrm{g} / \mathrm{mL}$ puromycin (Sigma-Aldrich, St. Louis, MO, United States).

\section{Time-Lapse Imaging of Live Cells}

Cells were seeded into the reservoirs of 2-well cell culture silicon inserts at a density of $3 \times 10^{4}$ cells/well (Ibidi, Martinsried, Germany). The inserts were designed to ensure directional cell migration, with a defined cell-free gap of $500 \mu \mathrm{m}$. Upon cellular attachment, the medium was replaced with serum-reduced medium for $24 \mathrm{~h}$ to suppress cell division. After nuclear staining with Hoechst $33342(0.5 \mu \mathrm{g} / \mathrm{mL})$ for $1 \mathrm{~h}$ and washing with PBS, the insert was removed and the migration of cells into the cell-free zone was screened. Time-lapse images were captured in $20 \mathrm{~min}$ intervals for $8 \mathrm{~h}$ at $37^{\circ} \mathrm{C}$ and $5 \% \mathrm{CO}_{2}$ using the PerkinElmer Operetta (PerkinElmer, Inc., Waltham, MA, United States) highcontent imaging system with a $20 \times$ objective $(20 \times$ long WD; $\mathrm{NA}=0.45$, working distance: $7.8 \mathrm{~mm}$; field of view: $675 \times 509$; depth of focus: $4.6 \mu \mathrm{m}$; optical xy resolution: $0.7 \mu \mathrm{m}$ ).

\section{Single-Cell Tracking of Cultured Myoblasts}

Time-lapse microscopy was used to quantify the migratory parameters. Single cells were tracked manually from frame to frame using the ImageJ (National Institutes of Health, Bethesda, MD, United States) ${ }^{1}$ and CellTracker ${ }^{2}$ (Piccinini et al., 2016) software programs. Nuclear tracking was used to follow the migration of individual cells. Dying or damaged cells were excluded from the analysis. The length of total path, maximum distance from the origin, as well as the average and maximum cell speeds were calculated. The vectorial distance of migration (i.e., real shift of the cell) from the origin was also quantified. Individual migratory tracks into the cell-free zone were visualized.

\section{Wound Scratch Assay}

For the wound scratch assay, cells were grown in 6-well plates until they reached confluence. After $24 \mathrm{~h}$ incubation in serumreduced medium, cell-free zones were created by scratching the cell layer with a P200 pipette tip. Images of the cell-free zone were captured immediately $(0 \mathrm{~h}), 4$ and $8 \mathrm{~h}$ after wounding, using a Leica DMil phase-contrast microscope (Leica Microsystems, Wetzlar, Germany). Between imaging periods, the cells were incubated at $37^{\circ} \mathrm{C}$ and $5 \% \mathrm{CO}_{2}$. The area of the cell-free zone was measured using Digimizer image analysis software (MedCalc Software bvba, Ostend, Belgium). The closure of the cell-free area was calculated as follows: (area of cell-free zone at $t_{0 h}$ - area of cell-free zone at $\left.\mathrm{t}_{x h}\right) /$ area of cell-free zone at $\mathrm{t}_{0 h}$.

\section{Fluorescence Staining}

For fluorescence cytochemistry, the cells subjected to wounding were fixed at indicated time points, stained with fluorescence

${ }^{1}$ https://imagej.nih.gov/ij/

${ }^{2} \mathrm{http}: / /$ celltracker.website/ markers, and studied to evaluate the migratory cells in the scratched area. For centrosome staining, cells were fixed with methanol 2, 4, and $6 \mathrm{~h}$ after scratching. After permeabilization with $0.5 \%$ Tween-20 (Sigma-Aldrich), the samples were blocked in $4 \%$ bovine serum albumin (BSA; Sigma-Aldrich), and stained with a mouse monoclonal anti- $\gamma$-tubulin antibody (1:200; SigmaAldrich) at $4{ }^{\circ} \mathrm{C}$ overnight, followed by incubation with an Alexa Fluor 488-conjugated anti-mouse secondary antibody (Jackson ImmunoResearch, Cambridgeshire, United Kingdom) a day later.

To visualize the actin filaments, cells subjected to the abovedescribed scratch assay were fixed with a methanol-free $4 \%$ formaldehyde solution (Thermo Fischer Scientific) $2 \mathrm{~h}$ after wounding. After permeabilization with $0.3 \%$ Triton X-100 (Sigma-Aldrich) and blocking in 4\% BSA (Sigma-Aldrich), the actin filaments were stained with Alexa Fluor 647-conjugated phalloidin (A22287, Thermo Fisher Scientific).

For syndecan-4 immunostaining, myoblasts were fixed with $4 \%$ formaldehyde solution $2 \mathrm{~h}$ after wounding, permeabilized with $0.3 \%$ Triton X-100, and blocked with 1\% BSA. Rabbit polyclonal anti-syndecan-4 primary antibody (immunogen: synthetic peptide surrounding amino acid 184 of human syndecan 4; PA1-32485; Invitrogen, Carlsbad, CA, United States) was visualized with the appropriate Alexa Fluor 568-conjugated (Invitrogen), or Alexa Fluor 488-conjugated secondary antibody (Jackson ImmunoResearch, Cambridgeshire, United Kingdom) secondary antibody. For double immunostaining experiments, cells were fixed with $4 \%$ formaldehyde solution, permeabilized with $0.1 \%$ Triton X-100 and blocked with 3\% BSA. Focal adhesions were marked with mouse monoclonal anti-FAK primary antibody (sc-271126; Santa Cruz Biotechnology, Dallas, TX, United States) and with Alexa Fluor 488-conjugated secondary antibody (Jackson ImmunoResearch, Cambridgeshire, United Kingdom). The cis-Golgi network was stained by mouse monoclonal anti-GM130 antibody (610822; BD Biosciences, San Jose, CA, United States), and followed by incubation with CF568-conjugated secondary antibody (Biotinum, Fremont, CA, United States). Nuclei were counterstained with Hoechst 33258 (0.01 mg/mL, Sigma-Aldrich).

\section{Quantification of Centrosome Positioning}

The positions of centrosomes were analyzed to quantify cell polarity, based on a previous characterization of centrosome reorientation in response to a scratch (Etienne-Manneville and Hall, 2001). Anti- $\gamma$-tubulin-stained samples were inspected and imaged using a Nikon Eclipse Ti-E microscope frame (Nikon Instruments Inc., Melville, NY, United States) with epifluorescent illumination using $20 \times$ objective (Nikon Plan fluor $20 \times$ DIC $\mathrm{N} 2$, NA = 0.50). The images were analyzed using ImageJ software.

Two hours after wounding, only the migrating cells next to the scratched area were analyzed. For selected cells adjacent to the cell-free zone, the direction of migration was designated as perpendicular to the wound edge, the nucleus was set as the origin, and a $30^{\circ}$ circular sector facing the direction of wound closure was assigned. Centrosomes situated within this assigned area were scored as correctly oriented. To monitor the time 
dependency of centrosome reorientation in different cell lines, the position of centrosomes was analyzed 2, 4, and $6 \mathrm{~h}$ after wounding in the 1 st and 2 nd row of myoblasts in the different cell lines along the wound edge based on the method described by Gotlieb et al. (1983). The position of centrosomes was considered "toward" the wound edge (between the nucleus and the wound edge), "middle" (along the side the nucleus), or "away" (between the nucleus the monolayer behind the cells).

\section{Super-Resolution dSTORM Imaging}

Super-resolution direct stochastic optical reconstruction microscopy (dSTORM) measurements were performed using a custom-made inverted microscope based on a Nikon Eclipse Ti-E frame. After conditioning (through spatial filtering via fiber coupling and beam expansion), the applied laser beams were focused into the back focal plane of the microscope objective (Nikon CFI Apo $100 \times, \mathrm{NA}=1.49$ ) to produce a collimated beam on the sample. The angle of illumination was then set through a tilting mirror mounted into a motorized gimbal holder and placed into the conjugate plane of the sample. All dSTORM images were captured under epi-illumination at an excitation wavelength of $634 \mathrm{~nm}$ (Thorlabs HL63133DG: $637 \mathrm{~nm}, \mathrm{P}_{\max }=170 \mathrm{~mW}$ in a Thorlabs TCLDM9 TE-Cooled mount set to $19^{\circ} \mathrm{C}$ ). The laser intensity was controlled via a Thorlabs LDC500 laser driver and set to an output of 2$4 \mathrm{~kW} / \mathrm{cm}^{2}$ on the sample plane. An additional laser (Nichia: $405 \mathrm{~nm}, \mathrm{P}_{\max }=60 \mathrm{~mW}$ ) was used for reactivation. Images were captured using an Andor iXon3 897 BV EMCCD digital camera (512 pixels $\times 512$ pixels; pixel size: $16 \mu \mathrm{m})$. The size of the illuminated sample region was matched to the size of the detector, which determined the field of view $\left(80 \times 80 \mu \mathrm{m}^{2}\right)$. Typically, the frame stacks for dSTORM super-resolution images were captured at a reduced image size (i.e., crop mode). A fluorescence filter set (Semrock, LF405/488/561/635-A-000 dichroic mirror with a BLP01647R-25 emission filter) was used to select and separate the excitation and emission lights in the microscope. During measurements, the perfect focus system of the microscope was used to maintain focus on the sample at a precision level of $<30 \mathrm{~nm}$. Immediately before measurement, the sample storage buffer was replaced with a GLOX switching buffer (van de Linde et al., 2011), and the sample was mounted on a microscope slide. During a typical imaging session, 20,000 frames were captured at an exposure time of 20 or $30 \mathrm{~ms}$. The image stacks were analyzed using rainSTORM localization software (Rees et al., 2013) and reconstructed using the built-in Simple Histogram method with a super-pixel size of $13.33 \mathrm{~nm}$. The Thompson-precision (Thompson et al., 2002) and PSF size acceptance ranges were set to $0-35 \mathrm{~nm}$ and $0.7-1.5$ pixels, respectively.

\section{Nanoscale Analysis of the Actin Cytoskeletal Structure}

After dSTORM imaging, phalloidin-stained samples were subjected to a nanoscale analysis of the actin cytoskeleton. The dSTORM images of lamellipodial actin structures were processed using ImageJ software. The super-resolution images were converted to grayscale, adjusted to a fixed threshold, and noise filtered. The ImageJ Skeletonize function was used to create binary skeletonized images. Then the Skeleton Analysis plugin was used to calculate the number of branches belonging to each skeleton in every image and to measure the length of each individual branch. To describe the difference between the cortical actin-rich region and the inner actin-depleted area of the lamellipodial actin network, three areas (each $126 \times 124$ px) were randomly selected in the external region (with a width of $350 \mathrm{px}$ beneath the plasma membrane) and three in the inner, internal region of the lamellipodia. Then the average number of branches and average length of the individual branches were measured in each of these selected rectangles and compared.

\section{Evaluation of Syndecan-4 Immunostaining}

Wide-field fluorescence images of syndecan-4 immunostained samples were acquired by a Nikon Eclipse Ti-E microscope (Nikon Instruments Inc.) with $40 \times$ (Nikon CFI Plan Fluor $40 \times, \mathrm{NA}=0.75)$ and $100 \times($ Nikon CFI Plan Apo DM Lambda $100 \times$ Oil, $\mathrm{NA}=1.45$ ) objectives, and pseudo-colored using ImageJ. The contours of the individual cells were drawn, and the average pixel intensity within the border of the cells were quantified following background correction. The intensity value of each pixel was measured within the selected area and the sum of the intensities was divided by the area of the cell to obtain the average syndecan- 4 intensity value of the individual cells. Furthermore, cells were partitioned into 4 quadrants considering the nucleus as the origin, a $90^{\circ}$ circular sector facing the direction of the wound closure was assigned, and the syndecan- 4 signal intensity within this area was quantified.

\section{Assessment of Intracellular $\mathrm{Ca}^{2+}$ Distribution}

As control, scrambled and two syndecan-4-targeted myoblast cell lines were seeded onto glass 8-well chambered coverslips (ibidi GmbH, Gräfelfing, Germany) at $1 \times 10^{4}$ cells/well density and grown for $24 \mathrm{~h}$ in serum-reduced medium. The confluent cultures were scratched as described above and further incubated for $2 \mathrm{~h}$. Subsequently, the cells were subjected to $2 \mu \mathrm{M}$ Fluo$4 \mathrm{AM}$ and $3 \mu \mathrm{M}$ Fura Red AM (Thermo Fisher Scientific) in serum-free D-MEM containing $50 \mu \mathrm{M}$ Verapamil (Sigma) for $30 \mathrm{~min}$ at $37^{\circ} \mathrm{C}$ and $5 \% \mathrm{CO}_{2}$. Verapamil was included to block the activity of multidrug transporters hindering effective dye loading. After several thorough washing steps, the green (493-572 $\mathrm{nm})$ and far red (609-797 $\mathrm{nm}$ ) fluorescence images were simultaneously acquired at 488 and $458 \mathrm{~nm}$ excitations, respectively, using a Zeiss 710 LSM laser scanning fluorescence confocal microscope with a Plan-Apochromat $40 \times($ N.A. $=1.4)$ oil immersion objective. The images were analyzed by Image $1.49 \mathrm{~g}$ software (National Institutes of Health, Bethesda, MD, United States). Ratio images were generated using the Ratio Plus Plug-in. For quantitative analysis, the Fluo-4 and Fura Red fluorescence intensities were determined along the axis of migrating cells starting from the leading edge. After background 
correction, ratios of green and red fluorescence were calculated. The slope of the intracellular $\mathrm{Ca}^{2+}$ distribution was determined by least squares method.

\section{Statistical Analysis}

Differences between groups were analyzed using a one-way ANOVA, followed by the Scheffe post hoc test or Student's $t$-test. GraphPad Prism 7.0 (GraphPad Software Inc., San Diego, CA, United States) was used for graphing and statistical analyses. The data are expressed as means + standard errors of the means. A $p<0.05$ was considered significantly different.

\section{RESULTS}

\section{Syndecan-4 Knockdown Decreases Directional Cell Migration}

Initially, we evaluated the expression of syndecan- 4 in $\mathrm{C} 2 \mathrm{C} 12$ myoblasts transfected stably with plasmids expressing shRNA specific for syndecan-4 (shSDC4\#1 and SDC4\#2 cell lines) using Western blotting technique. A more significant reduction in syndecan-4 expression was observed in shSDC4\#1 cells vs. shSDC4\#2 cells, whereas the scrambled sequence had no effect on syndecan-4 level (Supplementary Figure 1).

We then measured the effect of syndecan- 4 knockdown on directional migration in vitro into cell-free zones created using cell culture inserts for an $8 \mathrm{~h}$ period (Supplementary Movies 1-4). During this analysis, we observed significant decreases in the length of total movement, the vectorial distance, the maximum distance from the origin, as well as the average and maximum cell speeds in both the shSDC4\#1 and shSDC4\#2 cell lines (Figure 1A), whereas no significant difference was observed between the non-transfected and scrambled cell lines (Figure 1A). Moreover, we observed a greater reduction in migratory parameters in shSDC4\#1 cells (Figure 1), consistent with the previous observation of greater syndecan- 4 suppression in this line. An evaluation of the migratory tracks of individual cells depicts the positions of the $\mathrm{x}$ and $\mathrm{y}$ coordinates corresponding to the paths taken by each cell during the indicated time (as z; Figure 1B). The migratory tracks of highly motile control cells crossed each other in the middle of the cell-free zone (black area in the center of each image), whereas those of syndecan- 4 knockdown cells hardly moved from the original $\mathrm{x}-\mathrm{y}$ positions during the $8 \mathrm{~h}$ experimental period. We then prepared histograms to depict the percentages of cells within each velocity range (Figure 1C). Notably, the histograms of the non-transfected and scrambled cells formed bell-shaped curves, whereas those of both silenced cell lines exhibited a left-skewed distribution suggesting the higher ratio of less motile cells.

Representative images in Figure 2A depict a scratch wound in a confluent culture at 0,4 , and $8 \mathrm{~h}$. Quantification of the wound closures revealed a reduced closure of the cell-free zone in both syndecan-4 knockdown lines (Figure 2B). No significant difference was observed between non-transfected and scrambled cells (Figure 2B).

\section{Syndecan-4 Affects the Nanoscale Architecture of the Actin Cytoskeleton, as Determined by Super-Resolution dSTORM}

Cell motility is regulated by both extracellular factors and internal signaling mechanisms, including actin cytoskeletal remodeling. As syndecan-4 plays a crucial role in the organization of the actin cytoskeleton (Baciu et al., 2000; Elfenbein and Simons, 2013; Cavalheiro et al., 2017), we evaluated actin filaments using wide-field fluorescence microscopy (Figures 3A,B,D,E,G,H,J,K) and single-molecule localization super-resolution dSTORM imaging (lower magnification: Figures 3A,D,G,J; higher magnification: Figures 3C,F,I,L). Notably, our super-resolution dSTORM images reveal the sub-diffraction structure of the actin cytoskeleton and enable a more sophisticated experimental comparison of control and syndecan-4 knockdown samples. The reduced fluorescence background and enhanced resolution enabled visualization of the orientations and densities of individual actin bundles.

Next, wound scratch assays were performed to study the lamellipodial actin networks in migrating cells. To prove the migratory phenotype of the cells next to the cellfree zone, we stained the focal adhesions by anti-FAK antibody in the different cell lines, and FAK-stained focal adhesions were observed at the end of the stress fibers (Supplementary Figure 2). Interestingly, both the size and the number of focal adhesions decreased in syndecan-4 knockdown cells (Supplementary Figure 2). The cells next to the scratched areas were analyzed after actin filament labeling of the samples. For every sample, a panoramic map of individual wide-field fluorescence images was generated to cover the whole area of cell culture around the scratch wound (Supplementary Figures 3-6), and the lamellipodia of the migrating cells next to the wound were analyzed by dSTORM. Representative areas of the panoramic maps are shown in Figures 3A,D,G,J. Notably, syndecan-4 silencing altered the organization of the actin cytoskeleton (Figure 3) by hindering the development of actin structures (Figures 3G-L). The non-transfected and scrambled cells exhibited well-developed actin filaments (Figures 3A-F), whereas this filamentous actin cytoskeletal structure was less pronounced, and the lamellipodial actin network was less organized in syndecan- 4 knockdown cells (Figures 3G-L). Next, dSTORM images of the actin cytoskeleton were converted to binary images (Figure $4 \mathrm{~A}$ ) and analyzed further to quantify nanoscale changes in the actin network (Figure 4B). An analysis of binary images of the lamellipodial actin filaments (Figure 4A) revealed decreases in both the number of branches and the lengths of individual branches in the lamellipodial actin networks of syndecan-4 knockdown cells (Figure 4C).

As the binary images suggested the presence of an actindepleted inner region some micrometers away from the leading edge in syndecan-4 knockdown cell lines, next we quantified the nanoscale changes of the cortical (external) and the inner area of the lamellipodial actin network in the cell lines (Figure 4D). Both the average number of 

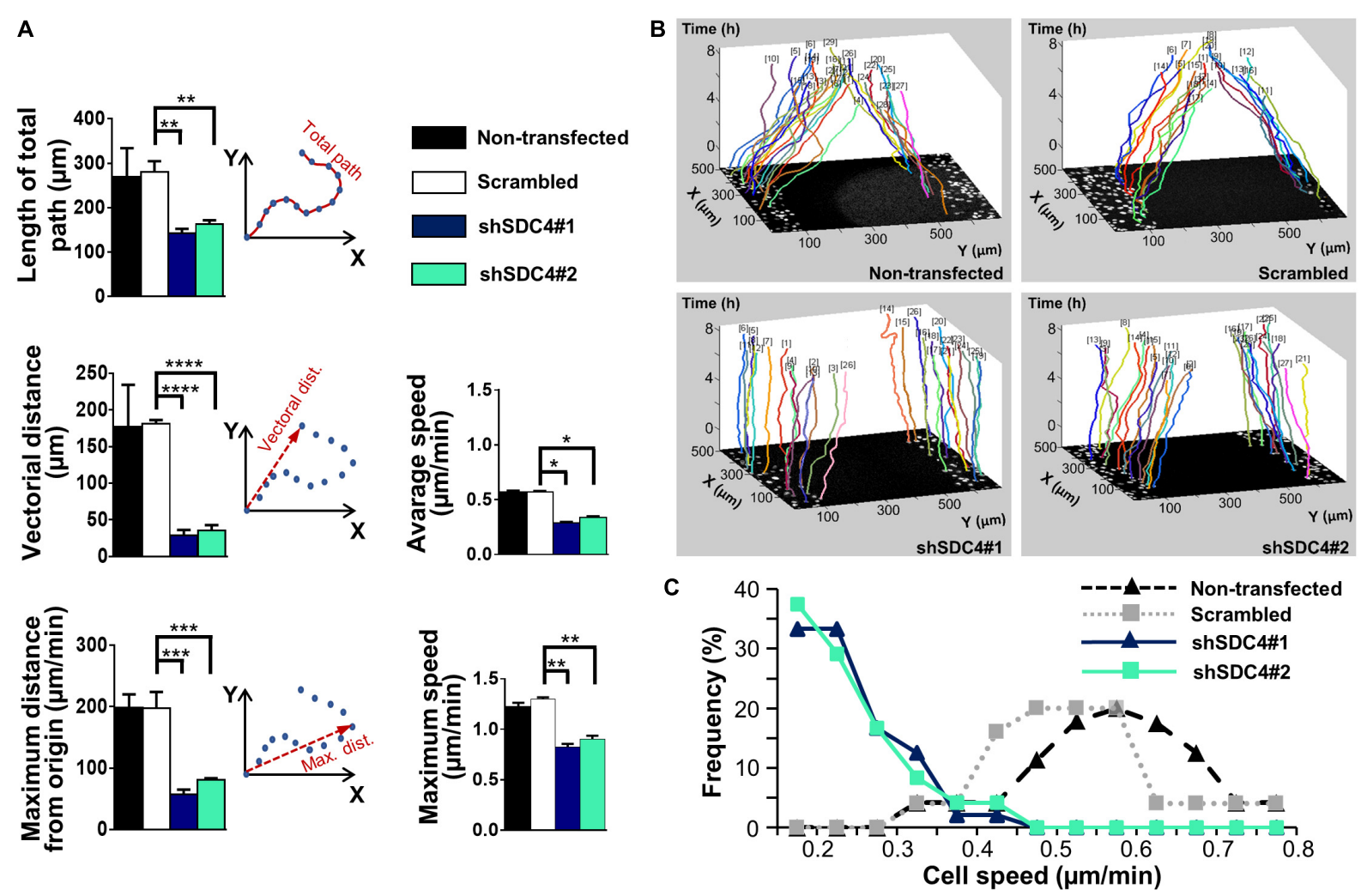

FIGURE 1 | The role of syndecan-4 in the directional migration of myoblasts. (A) Migration of non-transfected, scrambled, and syndecan-4-silenced (shSDC4\#1 and shSDC4\#2) C2C12 myoblasts to a cell-free zone was assessed after the removal of a cell culture insert. The total length of movement, maximum distance from the starting point, vectorial distance (i.e., real displacement of the cells), and the average and maximum cell speeds during directional migration are presented. The total duration of live cell microscopy was $8 \mathrm{~h}$, at a frame rate of 3/1 h. Four independent experiments were conducted, with 60-87 cells/cell line and 5-6 fields of view/experiment. Data are presented as means + standard errors of the means; ${ }^{\star} p<0.05$, ${ }^{* \star} p<0.01$, ${ }^{\star \star \star} p<0.001$, and ${ }^{* \star \star *} p<0.0001$. (B) Representative three-dimensional migration tracks. Different colors represent the total migrations of individual myoblasts; $x$ and $y$ axes: position of the cell ( $\mu \mathrm{m}), z$-axis: time ( $h$ ). (C) Histograms depict the distributions of cells from different lines according to cell speed (intervals of $0.05 \mu \mathrm{m} / \mathrm{min}$ ). The frequencies of cells from each line with average speeds within each interval were evaluated and are presented on the $y$-axis.

branches (in each skeleton) and the lengths of individual branches decreased in the inner region as compared to the external region of the lamellipodia in syndecan-4 knockdown cells, indicating the inhomogeneous lamellipodial actin structure in these cells (i.e., actin-rich external region and actindepleted inner area).

\section{Syndecan-4 Affects Centrosome Positioning and Cell Polarity}

Appropriate polarization of the cell (Lauffenburger and Horwitz, 1996), adequate positioning of the cellular compartments (Petrie et al., 2009), and dynamic reconstruction of the actin cytoskeleton (Gardel et al., 2010; Parsons et al., 2010) are required for efficient cell migration. As syndecan-4 silencing was shown to reduce myoblast migration, we next studied the polarization of syndecan-4 knockdown cells using centrosome localization, an indicator of cell polarity in migrating cells (Etienne-Manneville and Hall, 2001; Zhang and Wang, 2017). Specifically, the exact positions of the centrosomes were observed on immunostained samples obtained 2, 4, and $6 \mathrm{~h}$ after a wound scratch assay (Figure 5A, Table 1, and Supplementary Figures 7-9). Fluorescence images were captured after centrosome (anti- $\gamma$ tubulin) staining and used to generate panoramic maps of the entire scratched area (Supplementary Figures 7-9).

Two h after wounding, cells adjacent to the cell-free area were investigated using the nuclei as the points of reference (i.e., origins). The areas around the nuclei were divided into $30^{\circ}$ sectors, and centrosomes located in the $30^{\circ}$ circular sector facing toward the cell-free area were considered properly oriented (Figure 5B). Figure 5C depicts the numbers of centrosomes in the various sectors from experiments involving the different cell lines. Notably, syndecan- 4 knockdown was associated with significantly fewer centrosomes in the $30^{\circ}$ circular sector facing toward the cell-free zone, indicating an improper reorientation of the centrosomes in these cells (Figures 5C,D). In contrast, nearly all centrosomes of the scrambled and non-transfected cells were localized to this $30^{\circ}$ circular sector facing toward the cell-free area, indicating precise and proper regulation of centrosome positioning in these controls (Figures 5C,D). There was no significant difference between the non-transfected and scrambled cells (Figure 5D). To analyze the time dependency 
A

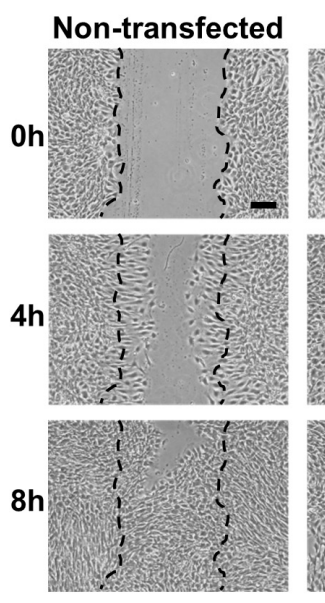

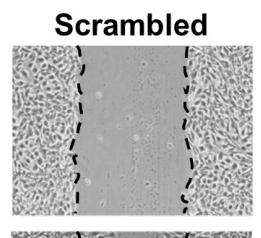
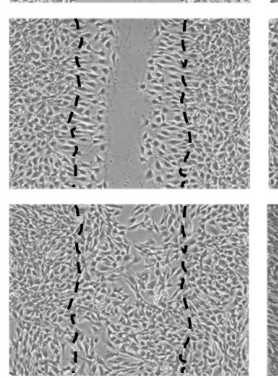
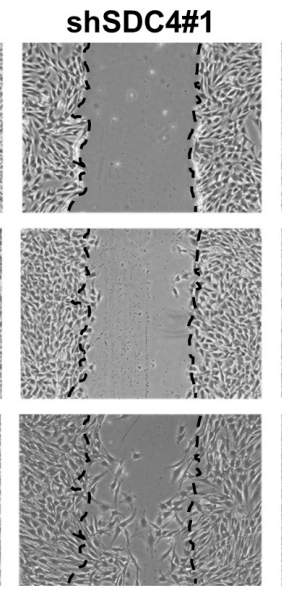

B

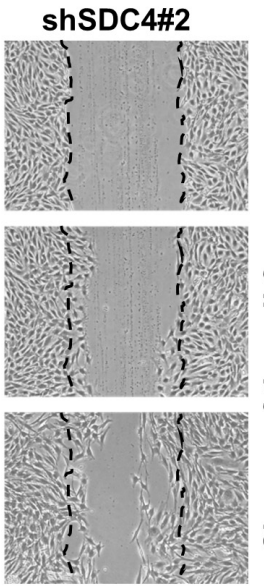

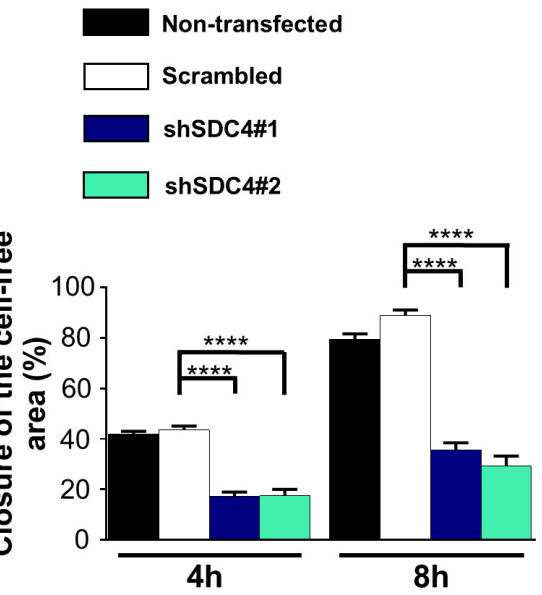

FIGURE 2 | Syndecan-4 influences the closure of the cell-free zone. (A) Representative microscopy images taken 0, 4, and 8 h after the initiation of a wound scratch assay. Dashed lines indicate the position of the cell-free zone at $0 \mathrm{~h}$. Scale bar: $200 \mu \mathrm{m}$. (B) Quantification of the closure of the cell-free area in cultures of non-transfected, scrambled, and syndecan-4-silenced (shSDC4\#1 and shSDC4\#2) cells; $n=4$ independent experiments. Data are shown as means + standard errors of the means; ${ }^{\star \star \star \star} p<0.0001$.

of centrosome reorientation, the position of centrosomes was studied 2, 4, and $6 \mathrm{~h}$ after wounding (Table 1). The number of centrosomes facing the wound edge increased in all cell lines during the $6 \mathrm{~h}$ period in both 1 st and 2nd row. Analysis of centrosome position along the wound edge revealed that in $83 \%$ of the scrambled cells in the first row the centrosomes were located toward the wound edge (between the nucleus and the wound edge) $2 \mathrm{~h}$ after wounding and $94 \%$ of the cells $6 \mathrm{~h}$ following wounding (Table 1). In contrast, only $25-27 \%$ of the syndecan-4 silenced cells presented centrosomes with "toward" position $6 \mathrm{~h}$ after wounding. In scrambled cells, only a few number of cells exhibited "middle" (along the side the nucleus), or "away" (between the nucleus the monolayer behind the cells) localized centrosomes $6 \mathrm{~h}$ after scratching. Based on these results, the reorientation of centrosomes during migration is delayed in syndecan-4 knockdown cells.

\section{Polarized Distribution of Syndecan-4 During Migration}

The former experiments demonstrated that syndecan-4 influences cellular polarity indicated by the impaired centrosome positioning and migration properties of myoblasts. Next we examined the intracellular distribution of syndecan-4 in control (scrambled) and syndecan-4 silenced cell lines in wide-field fluorescence images. According to immunostaining experiments, the amount of syndecan-4, considering all fluorescence signal intensities, was significantly higher in control cells than in syndecan-4 silenced cell lines (Figures 6A,B). Syndecan-4 accumulates in the quadrant of the migrating cells facing the wounded area (Figure 6A) which points the direction of migration (Figure 6C). Comparing the amount of syndecan4 accumulated in the quadrant facing the wounded area (Figure 6C) to the total of syndecan-4 level of the cells did not depict significant difference between the cell lines (Figure 6D).
Based on these results, the distribution of syndecan- 4 does not change as a result of silencing; only the total amount of syndecan-4 is lower in knockdown cells.

Since the wide-field images showed cytoplasmic syndecan- 4 staining, next we performed confocal imaging. The representative confocal image (Figure 6E) depicts the weak cell membrane localization of syndecan-4 in a migrating cell. Since earlier we showed the co-localization of syndecan- 4 with the antiGM130 Golgi marker and syndecan-4 is a member of focal adhesions, next we tested the co-distribution of syndecan-4 with FAK and GM130 (Figures 6F,G). The observed localization of syndecan-4 in the focal adhesions and cis-Golgi (Figures 6F,G) can explain the vacuolar and punctate signals of syndecan-4 staining. Moreover, earlier we have shown that the phospho(Ser179 in human, Ser183 in mouse) syndecan-4 accumulates in the cytoplasm during cytokinesis (Keller-Pinter et al., 2010). Therefore, we cannot exclude, that the syndecan-4 signal in our migrating cells partially originates from the cytoplasmic phosphorylated form.

\section{Syndecan-4 Knockdown Abrogates the Intracellular $\mathrm{Ca}^{2+}$ Gradient in Migrating Cells}

Normally, migrating cells exhibit a gradual increase in $\mathrm{Ca}^{2+}$ levels along the axis of migration. Accordingly, we next assessed the distribution of intracellular $\mathrm{Ca}^{2+}$ in syndecan-4-silenced $\mathrm{C} 2 \mathrm{C} 12$ cells and compered to that seen in cells transfected with a scrambled target sequence. The front-rear $\mathrm{Ca}^{2+}$ distribution was studied in cells adjacent to the cell-free area in a scratch-wounded confluent culture (Figure 7A). As expected, the intracellular $\mathrm{Ca}^{2+}$ concentration increased from the leading edge to the rear in control scrambled cells in (Figures 7B,C). In contrast, this $\mathrm{Ca}^{2+}$ gradient was completely abolished in syndecan-4-knockdown cells (Figures 7B,C). Since it has been reported that Fura Red 

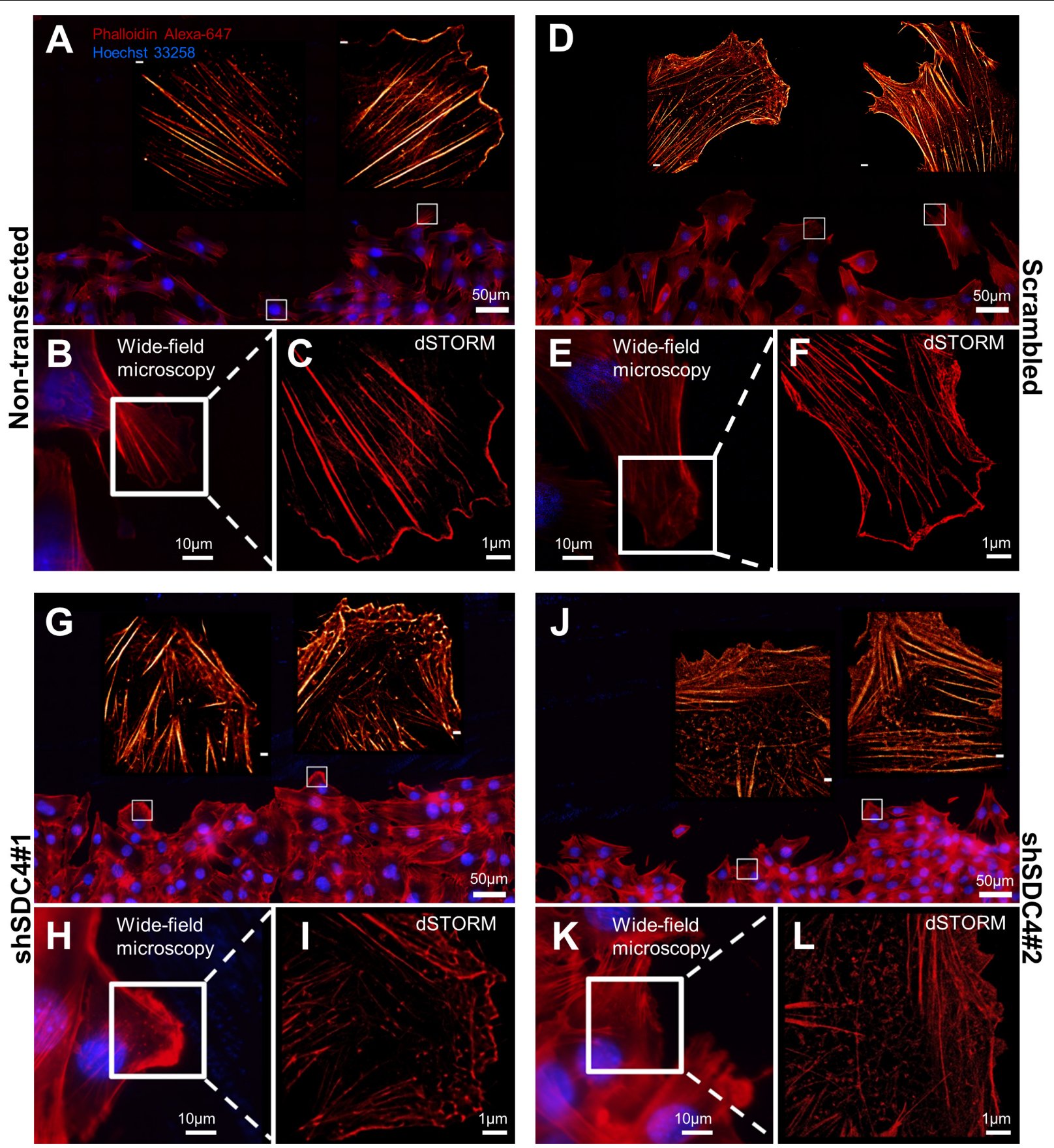

FIGURE 3 | Direct stochastic optical reconstruction microscopy (dSTORM) analysis of the actin cytoskeleton after syndecan-4 silencing. Representative wide-field fluorescence and super-resolution dSTORM images depict the actin skeletons of the cells adjacent to the cell-free zone in cultures of non-transfected (A-C), scrambled (D-F), shSDC4\#1 (G-I), and shSDC4\#2 (J-L) cell lines. Confluent monolayers were subjected to wound scratching. The cells were fixed 2 h later, and the actin filaments were stained with Alexa Fluor 647-conjugated phalloidin (red). Wide-field fluorescence images were obtained around the cell-free zone (A,D,G,J, higher magnification: $\mathbf{B}, \mathbf{E}, \mathbf{H}, \mathbf{K})$. Full panoramic maps of the scratched areas are shown in Supplementary Figures 3-6. The insets of the wide-field fluorescence images depict dSTORM images of the lamellipodial regions of migrating cells adjacent to the cell-free zone (A,B,D,E,G,H,J,K). Representative dSTORM images of lamellipodial actin structures are embedded in the original low-magnification images (A,D,G,J; bar: $1 \mu \mathrm{m})$ or are shown in separate higher magnification panels (C,F,I,L). Nuclei are stained by Hoechst 33258 (blue).

tend to accumulate in the mitochondria (Thomas et al., 2000), we explored whether the punctate structures can be observed in the $\mathrm{Ca}^{2+}$ indicator-loaded cell are mitochondria. Either control or syndecan-4-silenced cells exhibited distinct distribution for the $\mathrm{Ca}^{2+}$ indicators and the mitochondrial dye MitoTracker Deep Red (Supplementary Figure 10), demonstrating that neither 
A
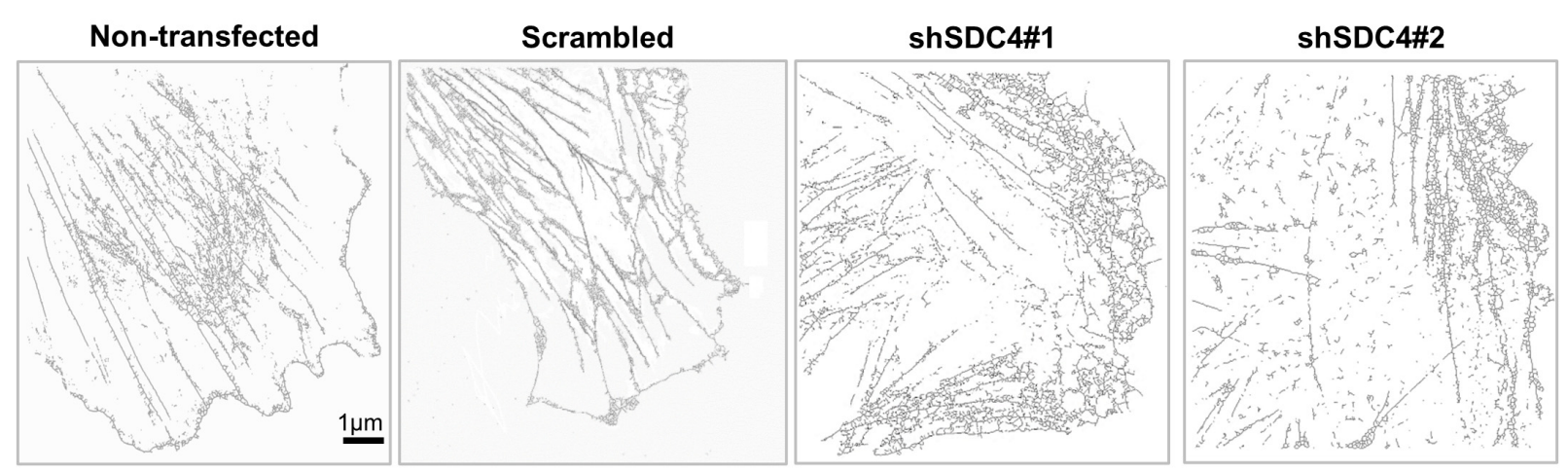

B

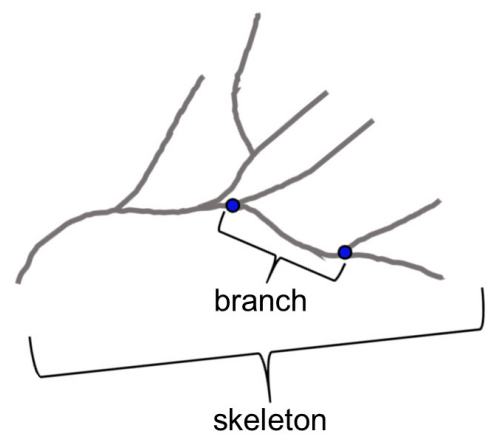

C

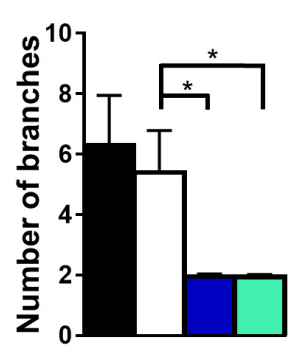

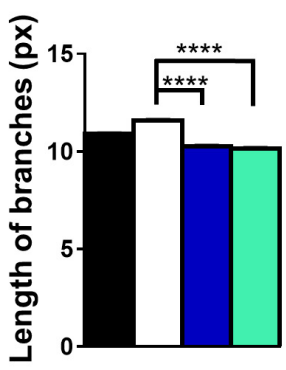

Non-transfected

Scrambled

shSDC4\#1

ShSDC4\#2

D
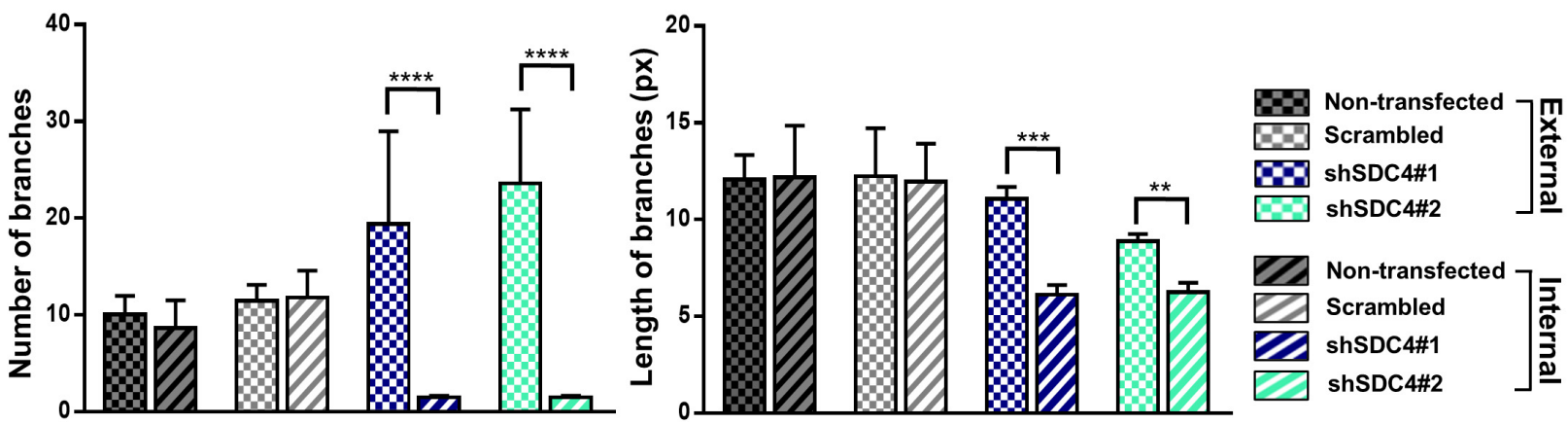

FIGURE 4 | Skeletal analysis of dSTORM images of the lamellipodial actin network. The phalloidin-stained lamellipodial actin cytoskeletons of non-transfected, scrambled, and syndecan-4-silenced (shSDC4\#1 and shSDC4\#2) cells were analyzed. Representative binary images converted from dSTORM images of the actin cytoskeleton are shown (A). Within the actin network, branching points divide the skeletons into smaller branches (B). The number of branches in the skeletons and the lengths of individual branches in the lamellipodial actin network in the whole binary images were quantified (C). To measure the differences between the external and the internal region of the lamellipodial actin network, the average number and length of branches were compared in randomly selected areas (three areas in both external and internal regions, each $126 \times 124$ pixels in size) of the binary images (D). Binary images of 5 cells/cell line were studied. Numbers of analyzed skeletons: 5,560-8,450/cell line; numbers of analyzed branches: 26,723-32,813/cell line. Number of analyzed skeletons in a single $126 \times 124$ pixels area: 2-69, number of analyzed branches in a single $126 \times 124$ pixels area: $32-336$. Data are shown as means + standard errors of the means; ${ }^{\star} p<0.05,{ }^{\star \star *} p<0.01,{ }^{* \star \star} p<0.001$, and ${ }^{* \star \star *} p<0.0001$.

Fluo-4 nor Fura Red accumulated in the mitochondria in our experiments. To exclude the possibility that alteration in the green and red fluorescence ratios is due to redistribution of organelles, in which one $\mathrm{Ca}^{2+}$ indicator accumulated more than the other, we performed an analysis, in which high intensity pixels (2.5-fold over mean cellular fluorescence) were omitted. Similar results were obtained this way to that shown in Figure 7 and Supplementary Figure 10, demonstrating that indeed the intracellular front-rear $\mathrm{Ca}^{2+}$ gradient was diminished by syndecan-4-silencing. In summary, our findings demonstrate the essential role of syndecan-4 in cell polarity.

\section{DISCUSSION}

Cell migration is an essential component of several physiological and pathological processes, including tissue regeneration. During regeneration of the skeletal muscle tissue, myoblasts (i.e., 
A

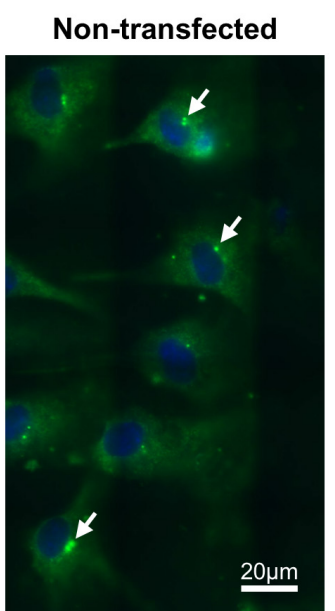

B



\section{Scrambled}

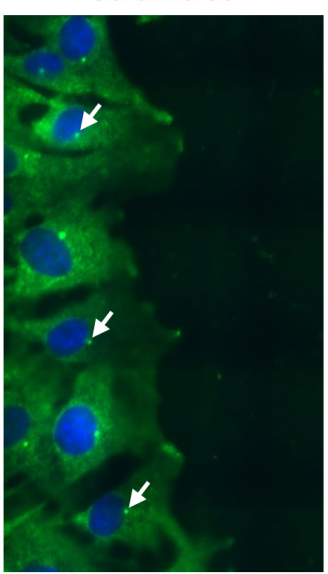

C

\section{Non-transfected}

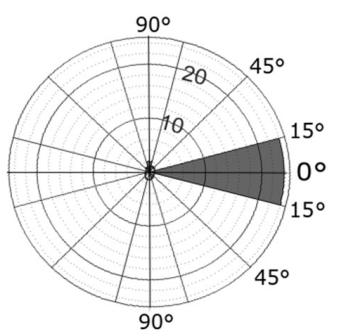

shSDC4\#1

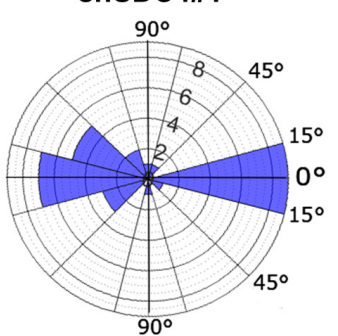

shSDC4\#1

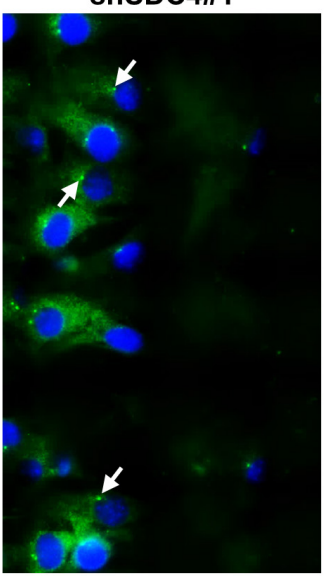

Scrambled

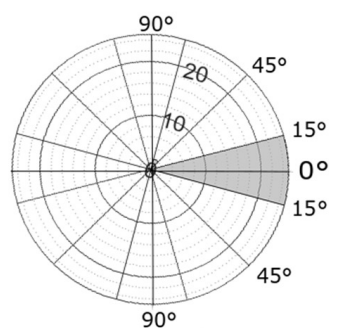

shSDC4\#2

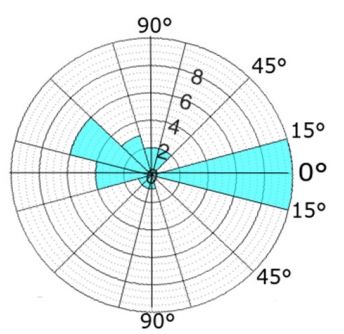

shSDC4\#2

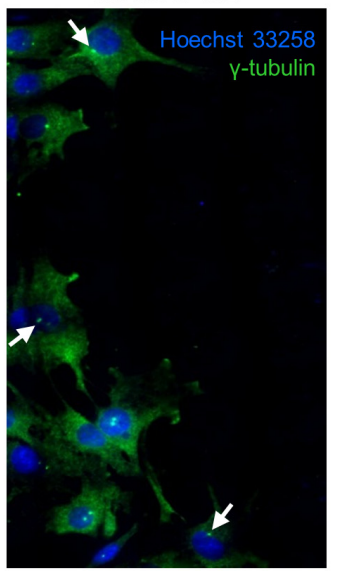

D
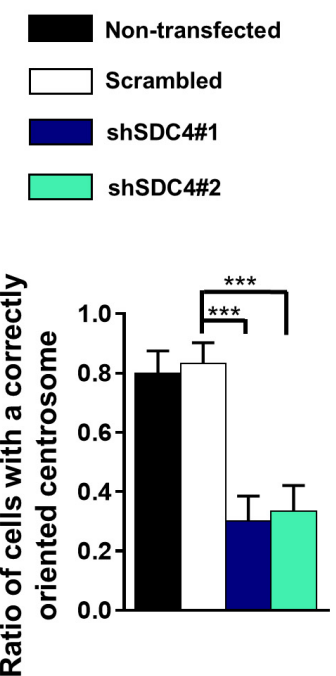

FIGURE 5 | Syndecan-4 affects centrosome positioning during migration. (A) Representative wide-field fluorescence images of the studied cell lines depict the positions of centrosomes $2 \mathrm{~h}$ after scratching. Anti- $\gamma$-tubulin-labeled centrosomes and Hoechst 33258-stained nuclei are shown in green and blue, respectively. Arrows indicate the centrosomes. (B) Schematic representation of a polarized migratory cell. To quantify the positions of centrosomes, the nucleus was set as the origin, and centrosomes located in the $30^{\circ}$ circular sector facing toward the direction of wound closure were considered properly located. (C) Pie charts (i.e., polar histograms) show the localization of centrosomes in different cell lines. The plane was partitioned into $30^{\circ}$ circular sectors with the nucleus as the origin. The radius of each circular sector represents the number of cells with centrosomes located in that $30^{\circ}$ sector. $N=3$ independent experiments. Thirty cells were analyzed per cell line. (D) Quantification of the results shown in (C). The graph presents the ratios of centrosomes in the $30^{\circ}$ sector facing the cell-free area. Data are shown as means + standard errors of the means; ${ }^{\star \star \star} p<0.001$.

activated satellite cells, skeletal muscle stem cells) proliferate, differentiate, migrate and fuse to form tubular, multi-nuclear myotubes. Accordingly, during muscle development and regeneration, myoblasts must be capable of migration to promote the cell-cell interactions and myoblast fusion required for muscle fiber formation. Syndecans, a family of transmembrane proteoglycans, have been reported to play crucial roles in tissue regeneration (Chung et al., 2016). We demonstrated previously that syndecan-4 could influence myoblast proliferation, as syndecan-4 silencing reduced cell cycle progression from the G1 to the $S$ phase and reduced the formation of mature myostatin, a negative regulator of muscle growth (Keller-Pinter et al., 2018). Syndecan-4 knockout mice also exhibited a decreased capacity for skin wound repair and angiogenesis (Echtermeyer et al., 2001), as well as inability to regenerate skeletal muscle following cardiotoxin-induced muscle necrosis (Cornelison et al., 2004). In summary, syndecan-4 appears to play an essential role in skeletal muscle development and regeneration, although the exact mechanism underlying this phenomenon remains unclear 
TABLE 1 | Comparison of the effect of syndecan-4 silencing on centrosome reorientation in the 1st and 2nd row of myoblasts along the wound edge.

\begin{tabular}{|c|c|c|c|c|c|c|c|c|c|c|c|c|}
\hline & \multicolumn{12}{|c|}{ Time after scratch } \\
\hline & \multicolumn{4}{|c|}{$2 \mathrm{~h}$} & \multicolumn{4}{|c|}{$4 \mathrm{~h}$} & \multicolumn{4}{|c|}{$6 \mathrm{~h}$} \\
\hline & $\begin{array}{c}\text { Non- } \\
\text { transfected }\end{array}$ & Scrambled & shSDC4\#1 & shSDC4\#2 & $\begin{array}{c}\text { Non- } \\
\text { transfected }\end{array}$ & Scrambled & shSDC4\#1 & shSDC4\#2 & $\begin{array}{c}\text { Non- } \\
\text { transfected }\end{array}$ & Scrambled & shSDC4\#1 & shSDC4\#2 \\
\hline \multicolumn{13}{|c|}{ Toward } \\
\hline 1st & $80 \pm 4.0$ & $83 \pm 1.0$ & $8 \pm 1.0$ & $7 \pm 1.5$ & $86 \pm 2.5$ & $88 \pm 1.0$ & $15 \pm 1.0$ & $14 \pm 2.0$ & $94 \pm 4.0$ & $92 \pm 3.0$ & $27 \pm 3.5$ & $25 \pm 3.5$ \\
\hline 2nd & $84 \pm 1.5$ & $87 \pm 2.5$ & $6 \pm 1.5$ & $4 \pm 0.5$ & $84 \pm 0.5$ & $89 \pm 5.0$ & $12 \pm 1.0$ & $16 \pm 2.0$ & $97 \pm 2.0$ & $98 \pm 1.5$ & $35 \pm 2.5$ & $27 \pm 2.5$ \\
\hline \multicolumn{13}{|c|}{ Middle } \\
\hline $1 \mathrm{st}$ & $8 \pm 1.0$ & $8 \pm 1.5$ & $22 \pm 2.0$ & $20 \pm 3.0$ & $2 \pm 4.5$ & $4 \pm 2.0$ & $17 \pm 2.5$ & $19 \pm 3.0$ & $2 \pm 2.0$ & $5 \pm 1.5$ & $16 \pm 1.5$ & $23 \pm 1.0$ \\
\hline 2nd & $6 \pm 0.5$ & $7 \pm 1.0$ & $18 \pm 3.0$ & $15 \pm 5.5$ & $7 \pm 6.5$ & $5 \pm 8.0$ & $21 \pm 1.0$ & $14 \pm 3.0$ & $2 \pm 2.5$ & $1 \pm 0.5$ & $17 \pm 2.5$ & $12 \pm 1.5$ \\
\hline \multicolumn{13}{|c|}{ Away } \\
\hline $1 \mathrm{st}$ & $12 \pm 3.0$ & $9 \pm 2.5$ & $70 \pm 3.0$ & $73 \pm 4.5$ & $12 \pm 2.0$ & $8 \pm 1.0$ & $71 \pm 1.5$ & $67 \pm 1.0$ & $4 \pm 2.0$ & $3 \pm 1.5$ & $57 \pm 2.0$ & $52 \pm 2.5$ \\
\hline 2nd & $10 \pm 1.0$ & $6 \pm 1.5$ & $76 \pm 1.5$ & $81 \pm 5.0$ & $9 \pm 6.0$ & $11 \pm 3.0$ & $67 \pm 2.0$ & $70 \pm 1.0$ & $1 \pm 0.5$ & $1 \pm 1.0$ & $52 \pm 5.0$ & $61 \pm 1.0$ \\
\hline
\end{tabular}

Four independent experiments, $n=100-100$ cells/cell lines in each row.

(Cornelison et al., 2004). Moreover, little is known about the specific role of syndecan-4 in mammalian myoblast migration.

Syndecan-4 was shown previously to affect migration in various cell types, including fibroblasts (Bass et al., 2007), endothelial cells (Chaudhuri et al., 2005), and hepatic stellate cells (Yin et al., 2017). This proteoglycan may also contribute to disease development by influencing the migration of tumor cells, such as lung adenocarcinoma (Toba-Ichihashi et al., 2016) and hepatoma (Charni et al., 2009); dendritic cells in the context of allergic rhinitis (Polte et al., 2015) and B-cells in the context of arthritis (Endo et al., 2015). A role for syndecan-4 has also been implicated in trophoblast migration and, consequently, the pathogenesis of preeclampsia (Jeyarajah et al., 2019). Importantly, Shin et al. (2013) reported that syndecan-4 overexpression increased the migration of turkey satellite cells and increased the activation of RhoA GTPase, and these motile phenomena required the cytoplasmic domain of syndecan-4. Other studies observed reduced motility following syndecan-4 knockdown in different cell types, consistent with our current observations, whereas high syndecan-4 level promoted migration (Toba-Ichihashi et al., 2016; Yin et al., 2017; Jeyarajah et al., 2019). Previous analyses of C2C12 mouse myoblast cells revealed that syndecan- 4 was the most prominent heparan sulfate proteoglycan in these cells when compared with syndecan1, syndecan-2, syndecan-3, glypican, or perlecan (Keller-Pinter et al., 2018), thus suggesting an important role for syndecan-4 in this cell type. However, the observed upregulation of syndecan-1, syndecan-2, and syndecan-3 mRNAs after syndecan-4 silencing (Keller-Pinter et al., 2018) suggests that other members of the syndecan family may compensate at least partially for the loss of syndecan-4. Given the importance of syndecan-4 in cell migration and cytoskeletal organization, we hypothesized that this proteoglycan would affect cellular polarity, centrosome positioning, and intracellular $\mathrm{Ca}^{2+}$ distribution during cell migration. We recently reported that syndecan-4 affects random migration and the directional persistence of migration in $\mathrm{C} 2 \mathrm{C} 12$ cells during $18 \mathrm{~h}$ movement (Becsky et al., 2020). Here we show the effect of syndecan- 4 silencing on $\mathrm{Ca}^{2+}$ distribution, centrosome positioning, and actin nanostructure after $8 \mathrm{~h}$ directional migration following wound scratching. Interestingly, the average speed values of the migrating $\mathrm{C} 2 \mathrm{C} 12$ cells were similar in the case of both random (Becsky et al., 2020) and directional migration.

Cell polarization and the associated rearrangement of the actin cytoskeleton and cell-matrix relationships are key factors in cell migration. In addition to the integrins, syndecan-4 plays a pivotal role in the formation of focal adhesions. Specifically, syndecan4 directly binds fibronectin to promote cell adhesions, thereby affecting cell migration, whereas the syndecan-4/PKC $\alpha /$ RhoA signaling axis promotes focal adhesion formation (Matthews et al., 2008; Yin et al., 2017). Furthermore, the downregulation of syndecan-4 was shown to suppress integrin-mediated cell adhesion by inhibiting FAK phosphorylation (Qin et al., 2017). Moreover, the cytoplasmic domain of syndecan-4 interacts directly with $\alpha$-actinin (Greene et al., 2003), leading to associations with other adhesion molecules, such as vinculin and zyxin (Cavalheiro et al., 2017), as well as the actin cytoskeleton (Choi et al., 2008). In a recent study on endothelial cells, syndecan-4 knockdown was shown to induce the decoupling of vinculin from F-actin filaments (Cavalheiro et al., 2017). Interestingly, the interaction of $\mathrm{PKC} \alpha$ and $\alpha$-actinin with syndecan-4 was shown to be reciprocal (Chaudhuri et al., 2005). Moreover, syndecan- 4 has been identified as a binding partner of dynamin II GTPase via its PH domain, and the resultant complex is a key regulator of focal adhesion and stress fiber formation in migrating cells (Yoo et al., 2005). Therefore, syndecan-4 serves as a central mediator in focal adhesion formation by bridging the interactions between integrins, fibronectin and intracellular molecules. Here we showed, that both the number and size of FAK stained focal adhesions were decreased in syndecan-4 knockdown cells during migration. Consequently, the loss of syndecan-4 would affect cell motility via multiple mechanisms, including the observed changes in the lamellipodial actin cytoskeletal structure.

As noted above, intracellular $\mathrm{Ca}^{2+}$ plays a crucial role in cell migration. Both $\mathrm{Ca}^{2+}$ influx from the extracellular 
A
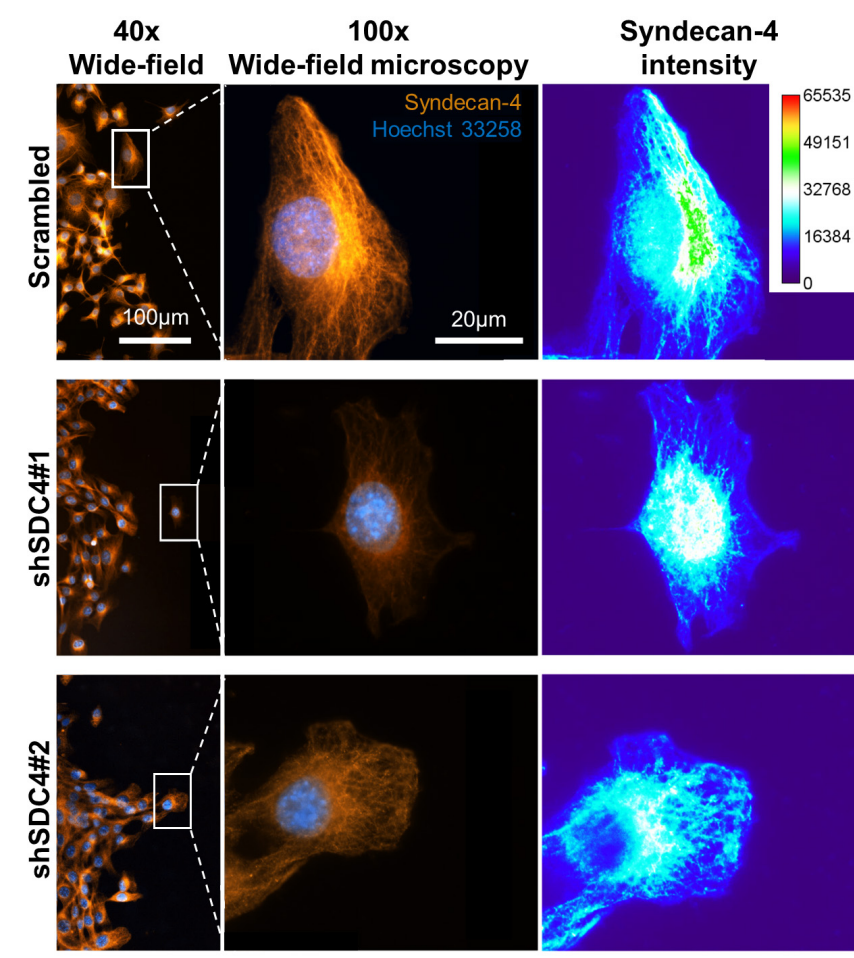

E
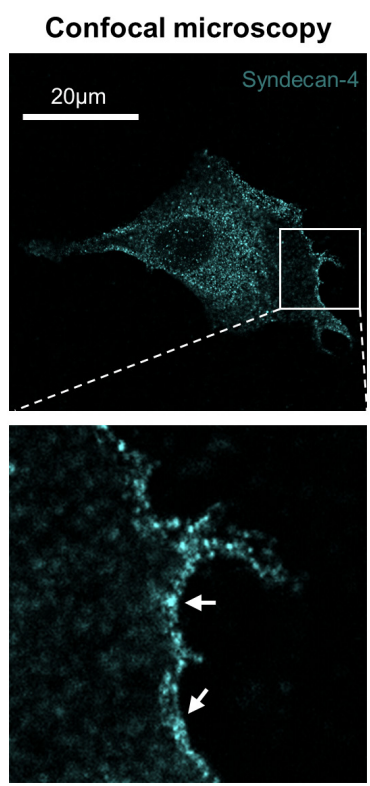

$\mathbf{F}$
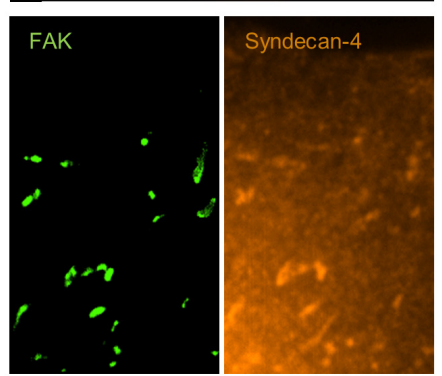

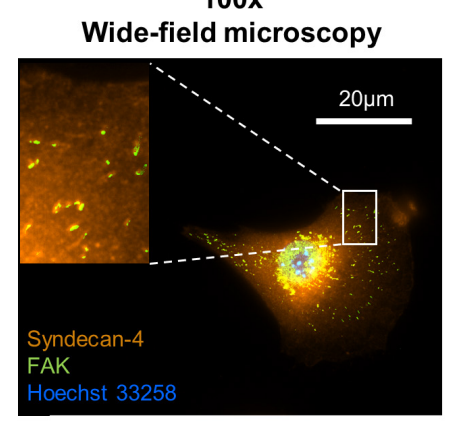

Syndecan-4 intensity
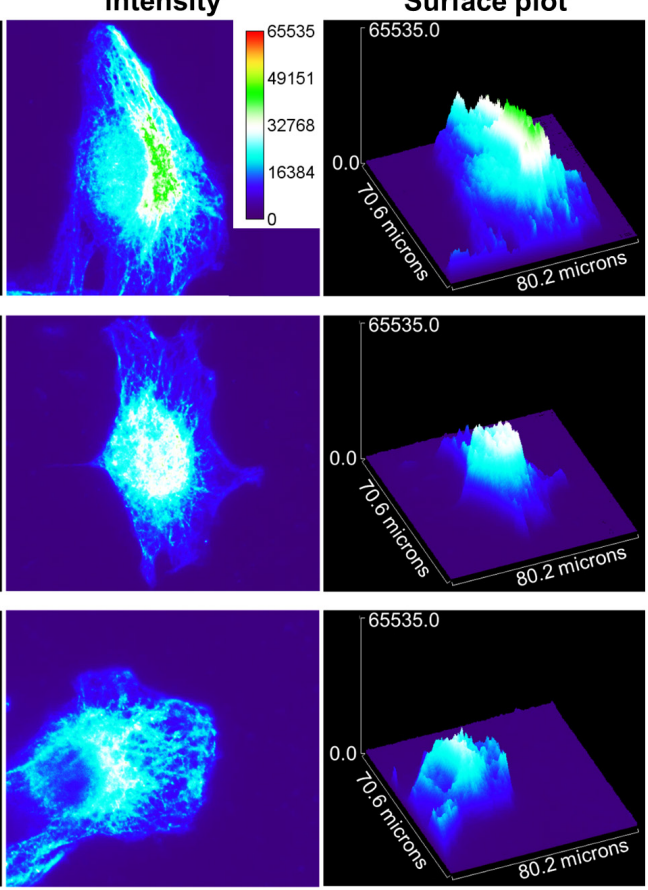

G Wide-field microscopy

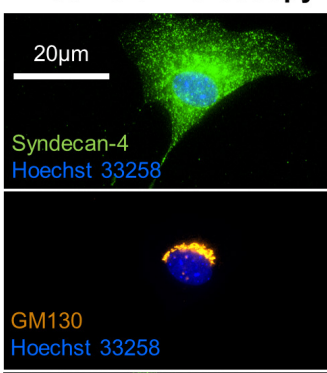

Merged
B

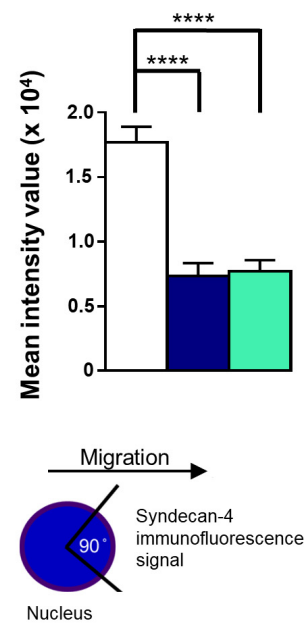

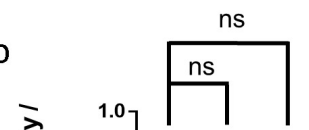
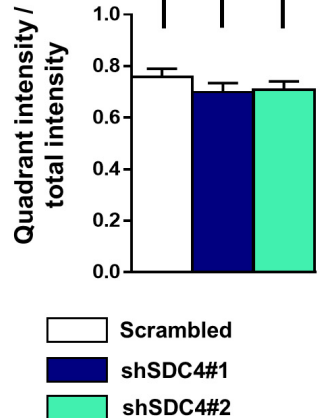

ShSDC4\#2


FIGURE 6 | Asymmetric distribution of syndecan-4 in migrating myoblasts. (A) Representative images show syndecan-4 distribution following staining with Alexa Fluor 568 fluorophore (orange). Nuclei are stained by Hoechst 33258 (blue). Representative pseudo-color images (2D and 3D) depict syndecan-4 signal intensity as indicated by the calibration bar. (B) The mean intensity values of the cells were quantified. (C) Cells were partitioned into 4 quadrants considering the nucleus as the origin; and a $90^{\circ}$ circular sector facing the direction of the wound closure was assigned and the syndecan- 4 signal intensity within this area was quantified. (D) The ratio of signal intensity of the quadrant pointing into the direction of migration (see schematic figure, $\mathbf{C}$ ), and the total syndecan-4 intensity of the cell was calculated 
FIGURE 6 | Continued

and compared in the different cell lines. Data are reported as means + standard errors of the means, $n=30$ cells/cell line were analyzed; ns: not significant; ${ }^{* \star \star *} p<0.0001$. (E) Representative confocal image depicts the cell membrane localization (arrows) of syndecan-4 in a migrating scrambled cell. (F) Representative wide-field fluorescence image of syndecan-4 and FAK staining in a migrating scrambled cell. (G) Representative wide-field fluorescence and confocal image of GM130 (cis-Golgi marker) and syndecan-4 double staining in migrating scrambled cells.

A



B

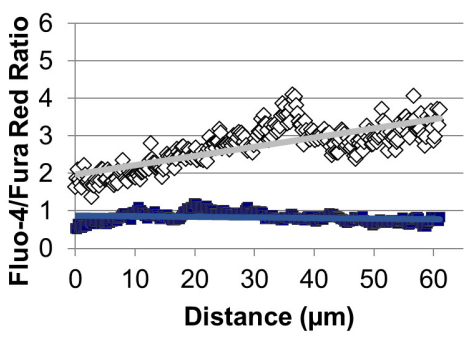

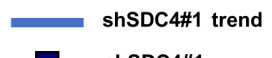

Scrambled
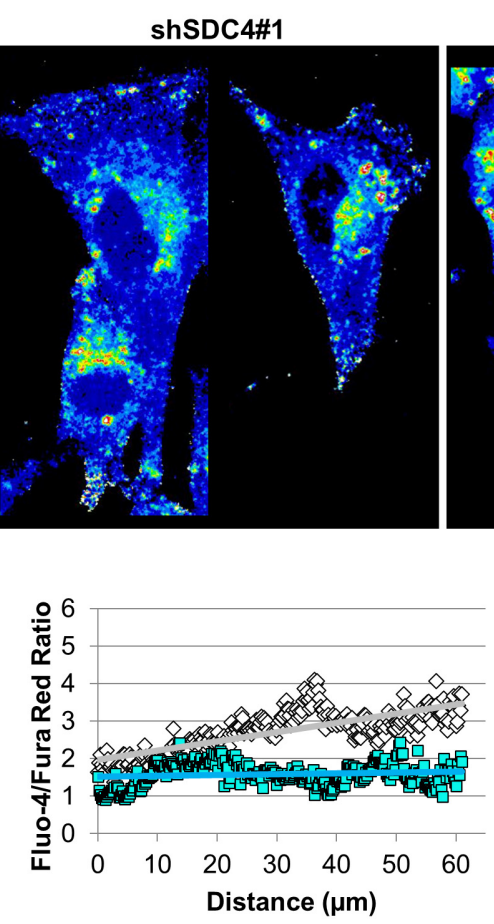

shSDC4\#2 trend

shSDC4\#2

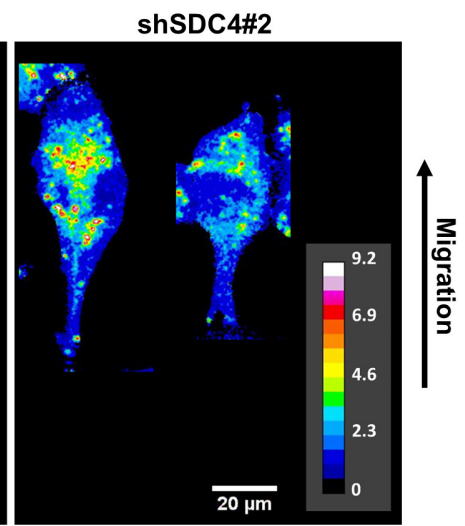

C

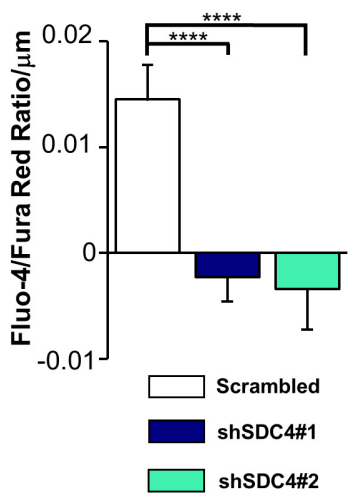

FIGURE 7 | Effect of syndecan-4 silencing on the distribution of intracellular $\mathrm{Ca}^{2+}$ in migrating myoblasts. (A) The ratio of Fluo-4 and Fura Red fluorescence, an indicator of the intracellular $\mathrm{Ca}^{2}+$ level, is shown in the representative pseudo-color images of scrambled, shSDC4\#1, and shSDC4\#2 cells. (B) The ratio of Fluo-4 and Fura Red fluorescence was determined along the migration axis from the leading edge to the rear of cells following scratch wounding. The mean fluorescence ratios are presented as a function of the distance from the leading edge. (C) The slopes of Fluo-4/Fura Red ratios along the migration axis in scrambled and syndecan-4 knockdown cells. Migrating cells next to the cell-free zones $(n=8-12)$ revealed that syndecan-4 knockdown completely abolished Ca ${ }^{2+}$ gradient development in migrating cells. Data are shown as the means + standard errors of the means; ${ }^{\star \star \star \star} p<0.0001$.

space via different plasma membrane $\mathrm{Ca}^{2+}$ channels and $\mathrm{Ca}^{2+}$ release from intracellular stores (primarily the endoplasmic reticulum) contribute to the cytosolic $\mathrm{Ca}^{2+}$ concentration. In addition to contractility, changes in the intracellular $\mathrm{Ca}^{2+}$ affect the activities of calmodulin-dependent enzymes and actincrosslinking proteins, thus playing a key role in the assembly of adhesions and junctions. Migrating cells establish a frontto-rear $\mathrm{Ca}^{2+}$ gradient, which increases toward the rear of the cell. Importantly, our findings suggest that syndecan-4 influences the development of this $\mathrm{Ca}^{2+}$ gradient, as demonstrated by its absence in syndecan-4 knockdown cells in association with decreased migration.

Syndecan-4 was shown earlier to influence $\mathrm{Ca}^{2+}$ concentrations in different cell types. In podocytes, syndecan-4 knockdown reduced the cell surface expression of the transient receptor potential cation channel subfamily C member (TRPC) 6 channel and consequently reduced the $\mathrm{Ca}^{2+}$ concentration (Liu et al., 2012). In contrast, another study of fibroblasts reported that the TRPC7 $\mathrm{Ca}^{2+}$ channel was more likely to be open in the absence of syndecan-4, resulting in an increased $\mathrm{Ca}^{2+}$ concentration (Gopal et al., 2015). However, a direct interaction has not been reported between syndecan-4 and TRPC7 (Afratis et al., 2017). Furthermore, the single knockdown of syndecan-4 in $\mathrm{HaCaT}$ keratinocytes did not affect the $\mathrm{Ca}^{2+}$ concentration, whereas the simultaneous silencing of both syndecan-1 and syndecan-4 decreased the cytosolic $\mathrm{Ca}^{2+}$ concentration in a TRPC4 channel-dependent manner (Gopal et al., 2015).

The development of $\mathrm{Ca}^{2+}$ gradient and the phosphorylation of FAK (Tyr397) are important for focal adhesion assembly and disassembly. Signaling via syndecan-4 is required for focal adhesion formation (Woods and Couchman, 2001), and syndecan-4 favors FAK phosphorylation (Wilcox-Adelman et al., 


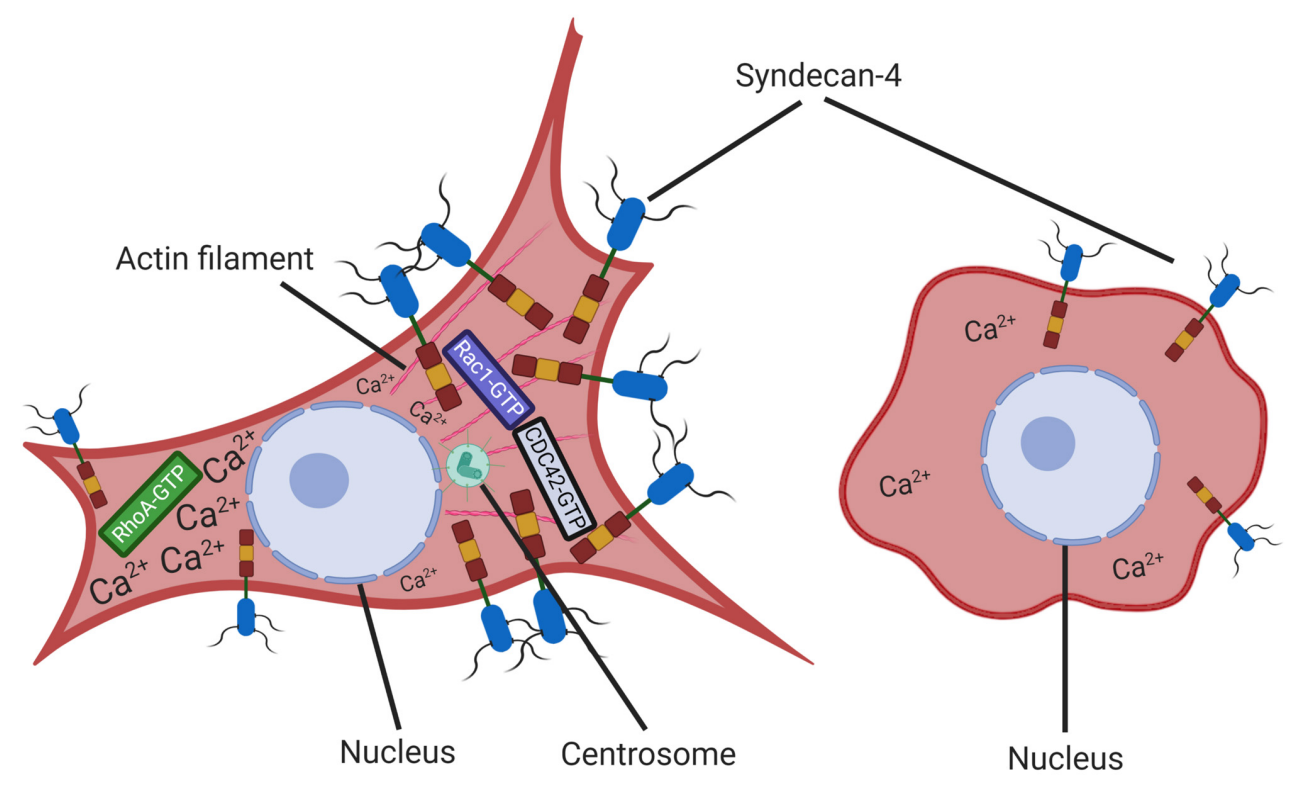

Control cell

Syndecan-4 knockdown cell

Migration

FIGURE 8 | Schematic representation of the effect of syndecan-4 knockdown on cell polarity and migration. The intracellular distribution of Ca ${ }^{2+}$ and syndecan-4 in non-transfected control (A) and syndecan-4 knockdown (B) myoblasts. In migrating cells, the formation of protruding leading edges are driven by Rac1/CDC42-GTPases favoring the generation of focal contacts, while the retraction and detachment occur at rear edges driven by RhoA-GTP. Syndecan-4 distributes asymmetrically in migrating cells; and syndecan-4 knockdown resulted in the improper positioning of centrosomes, the absence of a front-rear Ca $2+$ gradient and disturbances in the nanoscale structures of the actin fibers. These abnormalities led to decreases in cell polarity and migration. The figure was created with BioRender.com.

2002). The accumulation of phospho-FAK on the frontal side has been investigated and demonstrated in previous studies (Swaney et al., 2006; Carey et al., 2016; Gonzalez Malagon et al., 2018). The polarized distribution of syndecan- 4 can affect both $\mathrm{Ca}^{2+}$ gradient and local phospho-FAK level. Furthermore, low amount of syndecan- 4 in the rear of the migrating cells can contribute to focal adhesion disassembly.

As noted above, the localization of the centrosome is an indicator of polarization in a migrating cell (Etienne-Manneville and Hall, 2001; Zhang and Wang, 2017). To our knowledge, our study is the first to evaluate the effects of syndecan4 on centrosome positioning, the $\mathrm{Ca}^{2+}$ gradient, and the consequent effects on cell polarity. In our previous report of the role of syndecan-4 in cytokinesis, we demonstrated the polarized distribution of the phospho-Ser179 syndecan4, which accumulated in the intercellular bridges during cytokinesis (Keller-Pinter et al., 2010). The role for syndecan4 in regulating the activity of RhoA and Racl had previously described (Bass et al., 2007; Keller-Pinter et al., 2017), which are crucial regulators of cell polarity. Here we demonstrated that syndecan-4 knockdown led to centrosome disorientation, which indicated improper cell polarization. Further studies are needed to determine the signaling processes leading to syndecan4-dependent centrosome orientation. As the orientation of the centrosome-nucleus axis depends on a balance of actinand microtubule-mediated forces (Elric and Etienne-Manneville, 2014), structural changes in the actin cytoskeleton may contribute to the observed mislocalization of centrosomes. Furthermore, changes in the quantity and, presumably, the localization of Rac1 GTPase in syndecan-4-knockdown cells may also affect centrosome positioning and polarity. The latter postulation is supported by an earlier observation that Racl activity and membrane protrusions are localized to the leading edges of migrating syndecan-4-sufficient cells, resulting in persistent migration, whereas syndecan-4-null cells migrate randomly (Bass et al., 2007).

The front-to-rear cell polarity required for migration depends on the activities of various members of the small GTPase Rho family. The rear of a migrating cell is defined by high levels of RhoA activity and subsequent actomyosin contractility, in addition to an increased $\mathrm{Ca}^{2+}$ concentration and the activation of $\mathrm{Ca}^{2+}$-dependent proteases required to cleave focal adhesion proteins. Interestingly, Tsai and colleagues suggested the presence of crosstalk between $\mathrm{Ca}^{2+}$ signaling and Rho GTPases that would 
coordinate the oscillations of these factors in the leading edges of migrating cells (Tsai et al., 2015). As noted, phospho-Ser179 syndecan-4 regulates both Rac1 GTPase activity (Keller-Pinter et al., 2017) and intracellular $\mathrm{Ca}^{2+}$ level (Gopal et al., 2015). It would be interesting to determine whether these processes are coordinated simultaneously by syndecan -4 during cell migration.

\section{CONCLUSION}

In conclusion, we have identified new effects of syndecan4 in the regulation of cell migration. Specifically, syndecan- 4 silencing greatly reduces the migratory abilities of myoblasts. Presumably, this effect is due to a disturbance in cell polarization, which can be inferred from the shift in centrosome positioning relative to the nucleus and the absence of the intracellular $\mathrm{Ca}^{2+}$ gradient (Figure 8). The reduced migration capability might also be attributed to changes in the nanoscale structure of the lamellipodial actin cytoskeleton and reductions in cell-matrix adhesions. Our findings therefore elucidate the multiple roles of syndecan-4 in myoblast cell migration, although these findings are likely applicable to other cell types, given the ubiquitous expression of syndecan-4. This increase in general knowledge about cell migration will likely facilitate the development of strategies for the further exploration of a wide range of physiological and pathological migratory processes.

\section{DATA AVAILABILITY STATEMENT}

All datasets presented in this study are included in the article/Supplementary Material.

\section{AUTHOR CONTRIBUTIONS}

AK-P and $\mathrm{LH}$ conceived and designed the experiments. DB, KS, TG, SG-N, AB, ZB, LH, and AK-P performed the experiments. $\mathrm{DB}, \mathrm{KS}, \mathrm{SG}-\mathrm{N}, \mathrm{LH}$, and AK-P analyzed the results. AK-P wrote the manuscript with inputs from DB, KS, SG-N, LH, TG, and ME. AK-P, ME, PH, LH, and LD edited the manuscript. DB and KS contributed equally to this work. AK-P was the principal investigator of the study. All authors contributed to the article and approved the submitted version.

\section{REFERENCES}

Afratis, N. A., Nikitovic, D., Multhaupt, H. A., Theocharis, A. D., Couchman, J. R., and Karamanos, N. K. (2017). Syndecans - key regulators of cell signaling and biological functions. FEBS J. 284, 27-41. doi: 10.1111/febs. 13940

Baciu, P. C., Saoncella, S., Lee, S. H., Denhez, F., Leuthardt, D., and Goetinck, P. F. (2000). Syndesmos, a protein that interacts with the cytoplasmic domain of syndecan-4, mediates cell spreading and actin cytoskeletal organization. J. Cell Sci. 113(Pt 2), 315-324.

Bass, M. D., Roach, K. A., Morgan, M. R., Mostafavi-Pour, Z., Schoen, T., Muramatsu, T., et al. (2007). Syndecan-4-dependent Rac1 regulation determines directional migration in response to the extracellular matrix. J. Cell Biol. 177, 527-538. doi: 10.1083/jcb.200610076

\section{FUNDING}

This research was supported by the National Research, Development and Innovation Office of Hungary [grant nos: GINOP-2.3.2-15-2016-00040 (MYOTeam), EFOP-3.6.216-2017-00006, NKFI FK 134684, and NKFI K 132446]. The work was further supported by the János Bolyai Research Scholarship of the Hungarian Academy of Sciences (to AK-P), UNKP-19-4-SZTE-23 New National Excellence Program of the Ministry for Innovation and Technology Sciences (to AK-P), the National Research, Development and Innovation Fund (grant no. OTKA_K 128123 to $\mathrm{LH}$ ), and was conducted with support from the Szeged Scientists Academy under the sponsorship of the Hungarian Ministry of Human Capacities (grant no. EMMI:137252/2018/INTFIN, to SG-N). The dSTORM measurements were funded by the Hungarian Brain Research Programme (grant no. 2017-1.2.1-NKP-2017-00002); the National Research, Development and Innovation Office of Hungary (grant nos. GINOP-2.3.2-15-2016-00036) and an EU-funded Hungarian Grant (grant no. EFOP-3.6.1-16-2016-00008). For live-cell microscopy imaging $\mathrm{AB}$ and $\mathrm{PH}$ acknowledge support from the LENDULET-BIOMAG Grant (grant no. 2018-342) and the European Regional Development Funds (grant nos. GINOP-2.3.2-15-2016-00006, GINOP-2.3.2-15-2016-00026, and GINOP-2.3.2-15-2016-00037).

\section{ACKNOWLEDGMENTS}

We thank Zita Makráné Felhõ, Lászlóné Csontos (University of Szeged) and Gyöngyi Bézsenyi (RCNS, Budapest) for their excellent technical assistance. We are also grateful to Fanni Gergely (Cancer Research UK, Cambridge) for her suggestions with centrosome staining.

\section{SUPPLEMENTARY MATERIAL}

The Supplementary Material for this article can be found online at: https://www.frontiersin.org/articles/10.3389/fcell.2020. 575227/full\#supplementary-material

Becsky, D., Gyulai-Nagy, S., Balind, A., Horvath, P., Dux, L., and Keller-Pinter, A. (2020). Myoblast migration and directional persistence affected by syndecan4-mediated tiam-1 expression and distribution. Int. J. Mol. Sci. 21:823. doi: 10.3390/ijms21030823

Carey, D. J. (1997). Syndecans: multifunctional cell-surface coreceptors. Biochem J. $327(\mathrm{Pt}$ 1), 1-16. doi: 10.1042/bj327 0001

Carey, S. P., Goldblatt, Z. E., Martin, K. E., Romero, B., Williams, R. M., and Reinhart-King, C. A. (2016). Local extracellular matrix alignment directs cellular protrusion dynamics and migration through Racl and FAK. Integr. Biol. 8, 821-835. doi: 10.1039/c6ib00030d

Cavalheiro, R. P., Lima, M. A., Jarrouge-Boucas, T. R., Viana, G. M., Lopes, C. C., Coulson-Thomas, V. J., et al. (2017). Coupling of vinculin to F-actin demands 
Syndecan-4 proteoglycan. Matrix Biol. 63, 23-37. doi: 10.1016/j.matbio.2016. 12.006

Charni, F., Friand, V., Haddad, O., Hlawaty, H., Martin, L., Vassy, R., et al. (2009). Syndecan-1 and syndecan-4 are involved in RANTES/CCL5-induced migration and invasion of human hepatoma cells. Biochim. Biophys. Acta 1790, 1314-1326. doi: 10.1016/j.bbagen.2009.07.015

Chaudhuri, P., Colles, S. M., Fox, P. L., and Graham, L. M. (2005). Protein kinase Cdelta-dependent phosphorylation of syndecan-4 regulates cell migration. Circ. Res. 97, 674-681. doi: 10.1161/01.RES.0000184667.82354.b1

Choi, Y., Kim, S., Lee, J., Ko, S. G., Lee, W., Han, I. O., et al. (2008). The oligomeric status of syndecan-4 regulates syndecan-4 interaction with alpha-actinin. Eur. J. Cell Biol. 87, 807-815. doi: 10.1016/j.ejcb.2008.04.005

Chung, H., Multhaupt, H. A., Oh, E. S., and Couchman, J. R. (2016). Minireview: syndecans and their crucial roles during tissue regeneration. FEBS Lett. 590, 2408-2417. doi: 10.1002/1873-3468.12280

Cornelison, D. D., Filla, M. S., Stanley, H. M., Rapraeger, A. C., and Olwin, B. B. (2001). Syndecan-3 and syndecan-4 specifically mark skeletal muscle satellite cells and are implicated in satellite cell maintenance and muscle regeneration. Dev. Biol. 239, 79-94. doi: 10.1006/dbio.2001.0416

Cornelison, D. D., Wilcox-Adelman, S. A., Goetinck, P. F., Rauvala, H., Rapraeger, A. C., and Olwin, B. B. (2004). Essential and separable roles for Syndecan-3 and Syndecan-4 in skeletal muscle development and regeneration. Genes Dev. 18, 2231-2236. doi: 10.1101/gad.1214204

Echtermeyer, F., Streit, M., Wilcox-Adelman, S., Saoncella, S., Denhez, F., Detmar, M., et al. (2001). Delayed wound repair and impaired angiogenesis in mice lacking syndecan-4. J. Clin. Invest. 107, R9-R14. doi: 10.1172/jci10559

Elfenbein, A., and Simons, M. (2013). Syndecan-4 signaling at a glance. J. Cell Sci. 126(Pt 17), 3799-3804. doi: 10.1242/jcs. 124636

Elric, J., and Etienne-Manneville, S. (2014). Centrosome positioning in polarized cells: common themes and variations. Exp. Cell Res. 328, 240-248. doi: 10.1016/ j.yexcr.2014.09.004

Endo, T., Ito, K., Morimoto, J., Kanayama, M., Ota, D., Ikesue, M., et al. (2015). Syndecan 4 Regulation of the development of autoimmune arthritis in mice by modulating B cell migration and germinal center formation. Arthritis Rheumatol. 67, 2512-2522. doi: 10.1002/art.39193

Etienne-Manneville, S., and Hall, A. (2001). Integrin-mediated activation of Cdc42 controls cell polarity in migrating astrocytes through PKCzeta. Cell 106, 489498. doi: 10.1016/s0092-8674(01)00471-8

Gardel, M. L., Schneider, I. C., Aratyn-Schaus, Y., and Waterman, C. M. (2010). Mechanical integration of actin and adhesion dynamics in cell migration. Annu. Rev. Cell Dev. Biol. 26, 315-333. doi: 10.1146/annurev.cellbio.011209.122036

Gonzalez Malagon, S. G., Lopez Munoz, A. M., Doro, D., Bolger, T. G., Poon, E., Tucker, E. R., et al. (2018). Glycogen synthase kinase 3 controls migration of the neural crest lineage in mouse and Xenopus. Nat. Commun. 9:1126. doi: 10.1038/s41467-018-03512-5

Gopal, S., Sogaard, P., Multhaupt, H. A., Pataki, C., Okina, E., Xian, X., et al. (2015). Transmembrane proteoglycans control stretch-activated channels to set cytosolic calcium levels. J. Cell Biol. 210, 1199-1211. doi: 10.1083/jcb. 201501060

Gotlieb, A. I., Subrahmanyan, L., and Kalnins, V. I. (1983). Microtubule-organizing centers and cell migration: effect of inhibition of migration and microtubule disruption in endothelial cells. J. Cell Biol. 96, 1266-1272. doi: 10.1083/jcb.96.5. 1266

Granes, F., Berndt, C., Roy, C., Mangeat, P., Reina, M., and Vilaro, S. (2003). Identification of a novel Ezrin-binding site in syndecan-2 cytoplasmic domain. FEBS Lett. 547, 212-216. doi: 10.1016/s0014-5793(03)00712-9

Greene, D. K., Tumova, S., Couchman, J. R., and Woods, A. (2003). Syndecan-4 associates with alpha-actinin. J. Biol. Chem. 278, 7617-7623. doi: 10.1074/jbc. M207123200

Hawke, T. J., and Garry, D. J. (2001). Myogenic satellite cells: physiology to molecular biology. J. Appl. Physiol. 91, 534-551. doi: 10.1152/jappl.2001.91.2. 534

Jeyarajah, M. J., Jaju Bhattad, G., Kops, B. F., and Renaud, S. J. (2019). Syndecan-4 regulates extravillous trophoblast migration by coordinating protein kinase $\mathrm{C}$ activation. Sci. Rep. 9:10175. doi: 10.1038/s41598-019-46599-6

Keller-Pinter, A., Bottka, S., Timar, J., Kulka, J., Katona, R., Dux, L., et al. (2010). Syndecan-4 promotes cytokinesis in a phosphorylation-dependent manner. Cell Mol. Life Sci. 67, 1881-1894. doi: 10.1007/s00018-010-0298-6
Keller-Pinter, A., Szabo, K., Kocsis, T., Deak, F., Ocsovszki, I., Zvara, A., et al. (2018). Syndecan-4 influences mammalian myoblast proliferation by modulating myostatin signalling and G1/S transition. FEBS Lett. 592, 31393151. doi: 10.1002/1873-3468.13227

Keller-Pinter, A., Ughy, B., Domoki, M., Pettko-Szandtner, A., Letoha, T., Tovari, J., et al. (2017). The phosphomimetic mutation of syndecan-4 binds and inhibits Tiam1 modulating Racl activity in PDZ interaction-dependent manner. PLoS One 12:e0187094. doi: 10.1371/journal.pone.0187094

Kim, J. M., Lee, M., Kim, N., and Heo, W. D. (2016). Optogenetic toolkit reveals the role of $\mathrm{Ca} 2+$ sparklets in coordinated cell migration. Proc. Natl. Acad. Sci. U.S.A. 113, 5952-5957. doi: 10.1073/pnas.1518412113

Koo, B. K., Jung, Y. S., Shin, J., Han, I., Mortier, E., Zimmermann, P., et al. (2006). Structural basis of syndecan-4 phosphorylation as a molecular switch to regulate signaling. J. Mol. Biol. 355, 651-663. doi: 10.1016/j.jmb.2005.09.087

Lauffenburger, D. A., and Horwitz, A. F. (1996). Cell migration: a physically integrated molecular process. Cell 84, 359-369. doi: 10.1016/s0092-8674(00) 81280-5

Liu, Y., Echtermeyer, F., Thilo, F., Theilmeier, G., Schmidt, A., Schulein, R., et al. (2012). The proteoglycan syndecan 4 regulates transient receptor potential canonical 6 channels via RhoA/Rho-associated protein kinase signaling. Arterioscler. Thromb. Vasc. Biol. 32, 378-385. doi: 10.1161/atvbaha.111.2 41018

Matthews, H. K., Marchant, L., Carmona-Fontaine, C., Kuriyama, S., Larrain, J., Holt, M. R., et al. (2008). Directional migration of neural crest cells in vivo is regulated by Syndecan-4/Rac1 and non-canonical Wnt signaling/RhoA. Development 135, 1771-1780. doi: 10.1242/dev.017350

Parsons, J. T., Horwitz, A. R., and Schwartz, M. A. (2010). Cell adhesion: integrating cytoskeletal dynamics and cellular tension. Nat. Rev. Mol. Cell Biol. 11, 633-643. doi: $10.1038 / \mathrm{nrm} 2957$

Petrie, R. J., Zhao, B., Bedford, F., and Lamarche-Vane, N. (2009). Compartmentalized DCC signalling is distinct from DCC localized to lipid rafts. Biol. Cell 101, 77-90. doi: 10.1042/bc20070108

Piccinini, F., Kiss, A., and Horvath, P. (2016). CellTracker (not only) for dummies. Bioinformatics 32, 955-957. doi: 10.1093/bioinformatics/btv686

Polte, T., Petzold, S., Bertrand, J., Schutze, N., Hinz, D., Simon, J. C., et al. (2015). Critical role for syndecan-4 in dendritic cell migration during development of allergic airway inflammation. Nat. Commun. 6:7554. doi: 10.1038/ncomms8554

Qin, Y., Zhu, Y., Luo, F., Chen, C., Chen, X., and Wu, M. (2017). Killing two birds with one stone: dual blockade of integrin and FGF signaling through targeting syndecan-4 in postoperative capsular opacification. Cell Death Dis. 8, e2920. doi: $10.1038 /$ cddis.2017.315

Rees, E. J., Erdelyi, M., Kaminski-Schierle, G. S., Knight, A. E., and Kaminski, C. F. (2013). Elements of image processing in localisation microscopy. J. Opt. 15:094012.

Ridley, A. J., Schwartz, M. A., Burridge, K., Firtel, R. A., Ginsberg, M. H., Borisy, G., et al. (2003). Cell migration: integrating signals from front to back. Science 302, 1704-1709. doi: 10.1126/science.1092053

Schultz, E., and McCormick, K. M. (1994). Skeletal muscle satellite cells. Rev. Physiol. Biochem. Pharmacol. 123, 213-257.

Shin, J., McFarland, D. C., and Velleman, S. G. (2013). Migration of turkey muscle satellite cells is enhanced by the syndecan- 4 cytoplasmic domain through the activation of RhoA. Mol. Cell Biochem. 375, 115-130. doi: 10.1007/s11010-0121534- 1

Swaney, J. S., Patel, H. H., Yokoyama, U., Head, B. P., Roth, D. M., and Insel, P. A. (2006). Focal adhesions in (myo)fibroblasts scaffold adenylyl cyclase with phosphorylated caveolin. J. Biol. Chem. 281, 17173-17179. doi: 10.1074/jbc. M513097200

Thomas, D., Tovey, S. C., Collins, T. J., Bootman, M. D., Berridge, M. J., and Lipp, P. (2000). A comparison of fluorescent $\mathrm{Ca} 2+$ indicator properties and their use in measuring elementary and global Ca2+ signals. Cell Calcium 28, 213-223. doi: $10.1054 /$ ceca.2000.0152

Thompson, R. E., Larson, D. R., and Webb, W. W. (2002). Precise nanometer localization analysis for individual fluorescent probes. Biophys. J. 82, 27752783. doi: 10.1016/s0006-3495(02)75618-x

Toba-Ichihashi, Y., Yamaoka, T., Ohmori, T., and Ohba, M. (2016). Up-regulation of Syndecan-4 contributes to TGF-betal-induced epithelial to mesenchymal transition in lung adenocarcinoma A549 cells. Biochem. Biophys. Rep. 5, 1-7. doi: 10.1016/j.bbrep.2015.11.021 
Tsai, F. C., Kuo, G. H., Chang, S. W., and Tsai, P. J. (2015). Ca2+ signaling in cytoskeletal reorganization, cell migration, and cancer metastasis. Biomed. Res. Int. 2015:409245. doi: 10.1155/2015/409245

van de Linde, S., Loschberger, A., Klein, T., Heidbreder, M., Wolter, S., Heilemann, M., et al. (2011). Direct stochastic optical reconstruction microscopy with standard fluorescent probes. Nat. Protoc. 6, 991-1009. doi: 10.1038/nprot.2011. 336

Vicente-Manzanares, M., Webb, D. J., and Horwitz, A. R. (2005). Cell migration at a glance. J. Cell Sci. 118(Pt 21), 4917-4919. doi: 10.1242/jcs.02662

Wilcox-Adelman, S. A., Denhez, F., and Goetinck, P. F. (2002). Syndecan-4 modulates focal adhesion kinase phosphorylation. J. Biol. Chem. 277, 3297032977. doi: 10.1074/jbc.M201283200

Woods, A., and Couchman, J. R. (2001). Syndecan-4 and focal adhesion function. Curr. Opin. Cell Biol. 13, 578-583. doi: 10.1016/s0955-0674(00) 00254-4

Xian, X., Gopal, S., and Couchman, J. R. (2010). Syndecans as receptors and organizers of the extracellular matrix. Cell Tissue Res. 339, 31-46. doi: 10.1007/ s00441-009-0829-3

Yin, L., Qi, Y., Xu, Y., Xu, L., Han, X., Tao, X., et al. (2017). Dioscin inhibits HSC-T6 cell migration via adjusting SDC-4 expression: insights from iTRAQbased quantitative proteomics. Front. Pharmacol. 8:665. doi: 10.3389/fphar. 2017.00665
Yoo, J., Jeong, M. J., Cho, H. J., Oh, E. S., and Han, M. Y. (2005). Dynamin II interacts with syndecan-4, a regulator of focal adhesion and stress-fiber formation. Biochem. Biophys. Res. Commun. 328, 424-431. doi: 10.1016/j.bbrc. 2004.12.179

Zhang, J., and Wang, Y. L. (2017). Centrosome defines the rear of cells during mesenchymal migration. Mol. Biol. Cell 28, 3240-3251. doi: 10.1091/mbc.E1706-0366

Zimmermann, P., and David, G. (1999). The syndecans, tuners of transmembrane signaling. FASEB J. 13(Suppl.), S91-S100. doi: 10.1096/fasebj.13.90 $01 . s 91$

Conflict of Interest: The authors declare that the research was conducted in the absence of any commercial or financial relationships that could be construed as a potential conflict of interest.

Copyright (ㄷ) 2020 Becsky, Szabo, Gyulai-Nagy, Gajdos, Bartos, Balind, Dux, Horvath, Erdelyi, Homolya and Keller-Pinter. This is an open-access article distributed under the terms of the Creative Commons Attribution License (CC BY). The use, distribution or reproduction in other forums is permitted, provided the original author(s) and the copyright owner(s) are credited and that the original publication in this journal is cited, in accordance with accepted academic practice. No use, distribution or reproduction is permitted which does not comply with these terms. 


\section{SUPPLEMENTARY METHODS}

\section{Western blotting}

Non-transfected, scrambled, and SDC4 knockdown (shSDC4\#1 and shSDC4\#2) cells were harvested in RIPA buffer $(20 \mathrm{mM}$ Tris- $\mathrm{HCl}$ pH 7.5, $150 \mathrm{mM} \mathrm{NaCl}, 1 \mathrm{mM}$ Na2EDTA, $1 \mathrm{mM}$ EGTA, 1\% NP-40, 1\% sodium deoxycholate, $2.5 \mathrm{mM}$ sodium pyrophosphate, $1 \mathrm{mM}$ glycerophosphate, $1 \mathrm{mM} \mathrm{Na} \mathrm{VO}_{4}, 1 \mu \mathrm{g}$ x ml leupeptin; Cell Signaling Technology, Danvers, MA, USA; \#9806) supplemented with $1 \mathrm{mM} \mathrm{Na-fluoride,} \mathrm{and} \mathrm{protease} \mathrm{inhibitor} \mathrm{cocktail}$ (Sigma-Aldrich). The samples were spun down at $18,927 \mathrm{x}$ g for $5 \mathrm{~min}$ at $4{ }^{\circ} \mathrm{C}$ to eliminate cellular debris, and the supernatants were separated by SDS/PAGE and blotted to nitrocellulose membrane. After blocking, membranes were incubated with primary antibodies including rabbit anti-SDC4 (PA1-32485; Pierce Protein Biology - Thermo Fisher Scientific, Waltham, Massachusetts, USA) and mouse anti-GAPDH (Cell Signaling Technology, \#2118, Danvers, Massachusetts, US). Following incubation with the appropriate horse-radish peroxidaseconjugated anti-IgG secondary antibodies (DAKO, Glostrup, Denmark), peroxidase activity was visualized by enhanced chemi9luminescent method (Advansta, Menlo Park, CA, USA). Quantification of signal intensity was performed by QUANTITY ONE software (Bio-Rad, Hercules, CA, USA).

\section{Immunostaining and quantification of focal adhesions}

For the staining of focal adhesions, cells were fixed with $4 \%$ formaldehyde solution 2 and $4 \mathrm{~h}$ after scratching. After permeabilization with 0.3\% Triton-X-100 (Sigma-Aldrich) and blocking in 3\% BSA (Sigma-Aldrich), focal adhesions were stained with anti-FAK primary antibody (sc271126; Santa Cruz Biotechnology, Dallas, TX, USA) and with an appropriate Alexa Fluor 488-conjugated secondary antibody (715-585-151; Jackson ImmunoResearch, Cambridgeshire, UK). Wide-field fluorescence images of FAK immunostained samples were acquired by a Nikon Eclipse Ti-E microscope (Nikon Instruments Inc.) with a 100× (Nikon CFI Plan Apo DM Lambda $100 \times$ Oil, NA = 1.45) objective, and processed using ImageJ software. The images were converted into binary images, particles in the range of 0 to $100 \mu \mathrm{m}^{2}$ were selected. The masks of these selected particles were constructed and the area of each particle was measured.

\section{Assessment of mitochondrial accumulation of $\mathrm{Ca}^{2+}$ indicators}

To explore the accumulation of $\mathrm{Ca}^{2+}$ indicators in the mitochondria, the cells were loaded by $\mathrm{Ca}^{2+}$ indicators as described in Materials and Methods, but in addition to the $\mathrm{Ca}^{2+}$ indicators, 100 nM MitoTracker Deep Red (Thermo Fisher Scientific) was included in the loading mixture. 
The far red fluorescence of MitoTracker Deep Red was acquired above $650 \mathrm{~nm}$ at $633 \mathrm{~nm}$ excitation. 


\section{SUPPLEMENTARY FIGURE LEGENDS}

Supplementary Figure 1. Effect of syndecan-4 silencing on C2C12 myoblasts. (A) Representative Western blot analysis depicts the level of syndecan-4 in cells stably expressing shRNA against syndecan-4 (shSDC4\#1, shSDC4\#2), or scrambled shRNA. GAPDH served as a loading control. (B) Quantification of syndecan-4 expression in the different cell lines. $\mathrm{n}=6$ independent experiments, means + standard errors of the means; $* *: p<0.01 ; *: p<0.05$.

\section{Supplementary Figure 2. Distribution of focal adhesions in control and syndecan-4-} silenced myoblasts. (A) Representative 100× wide-field fluorescence images depict the actin cytoskeleton (Alexa Fluor 647-conjugated phalloidin: red) and focal adhesion distribution (antiFAK antibody: green) of the migrating cells in different cell lines $2 \mathrm{~h}$ after wounding. Nuclei are stained by Hoechst 33258 (blue). To quantify focal adhesions, the focal adhesions were assigned in FAK stained fluorescence images. The area and the number of the focal adhesions were quantified 2 and $4 \mathrm{~h}$ after wounding the monolayer $(\mathbf{B}, \mathbf{C})$. Data are reported as means + standard errors of the means, $n=10$ cells/cell line, 4 independent experiments were analyzed; *: $\mathrm{p}<0.05 ; * *$ : $\mathrm{p}<0.01$.

\section{Supplementary Figure 3. Actin cytoskeletal structure of non-transfected C2C12} myoblasts around the cell-free zone. A representative wide-field fluorescence microscopy image depicts the actin skeletons of non-transfected C2C12 myoblasts around the cell-free zone. The inserts depict the lamellipodial actin structures in the migrating cells, which were imaged using dSTORM. The cells were fixed $2 \mathrm{~h}$ after confluent cultures were scratched, and the actin filaments were stained with Alexa Fluor 647-conjugated phalloidin (red). Nuclei are shown in blue (Hoechst 33258).

\section{Supplementary Figure 4. Actin cytoskeletal structure of scrambled myoblasts around the} cell-free zone. A representative wide-field fluorescence microscopy image depicts the actin skeletons of scrambled myoblasts around the cell-free zone. The inserts depict the lamellipodial actin structures in the migrating cells, which were imaged using dSTORM. The cells were fixed $2 \mathrm{~h}$ after confluent cultures were scratched, and the actin filaments were stained with Alexa Fluor 647-conjugated phalloidin (red). Nuclei are shown in blue (Hoechst 33258). 


\section{Supplementary Figure 5. Actin cytoskeletal structure of shSDC4\#1 myoblasts around the}

cell-free zone. A representative wide-field fluorescence microscopy image depicts the actin skeletons of shSDC4\#1 (i.e., syndecan-4 knockdown) myoblasts around the cell-free zone. The inserts depict the lamellipodial actin structures in the migrating cells, which were imaged using dSTORM. The cells were fixed $2 \mathrm{~h}$ after confluent cultures were scratched, and the actin filaments were stained with Alexa Fluor 647-conjugated phalloidin (red). Nuclei are shown in blue (Hoechst 33258).

\section{Supplementary Figure 6. Actin cytoskeletal structure of shSDC4\#2 myoblasts around the} cell-free zone. A representative wide-field fluorescence microscopy image depicts the actin skeletons of shSDC4\#2 (i.e., syndecan-4 knockdown) myoblasts around the cell-free zone. The inserts depict the lamellipodial actin structures in the migrating cells, which were imaged using dSTORM. The cells were fixed $2 \mathrm{~h}$ after confluent cultures were scratched, and the actin filaments were stained with Alexa Fluor 647-conjugated phalloidin (red). Nuclei are shown in blue (Hoechst 33258).

Supplementary Figure 7. Localization of centrosomes around the cell-free zone in the different cell lines $\mathbf{2}$ h after wounding. Representative wide-field fluorescence microscopy images depict the wounded areas in confluent cultures of non-transfected, scrambled and shSDC4\#1 and shSDC4\#2 (i.e. syndecan-4 knockdown) cell lines. The cells were fixed $2 \mathrm{~h}$ after the confluent cultures were scratched, and the centrosomes were stained with anti $\gamma$-tubulin (green). Nuclei are shown in blue (Hoechst 33258). Individual wide-field fluorescence images were obtained and used to generate panoramic maps for a further analysis of centrosome localization.

Supplementary Figure 8. Localization of centrosomes around the cell-free zone in the different cell lines $4 \mathrm{~h}$ after wounding. Representative wide-field fluorescence microscopy images depict the wounded areas in confluent cultures of non-transfected, scrambled and shSDC4\#1 and shSDC4\#2 (i.e. syndecan-4 knockdown) cell lines. The cells were fixed $4 \mathrm{~h}$ after the confluent cultures were scratched, and the centrosomes were stained with anti $\gamma$-tubulin (green). Nuclei are shown in blue (Hoechst 33258). Individual wide-field fluorescence images were obtained and used to generate panoramic maps for a further analysis of centrosome localization. 
Supplementary Figure 9. Localization of centrosomes around the cell-free zone in the different cell lines $6 \mathrm{~h}$ after wounding. Representative wide-field fluorescence microscopy images depict the wounded areas in confluent cultures of non-transfected, scrambled and shSDC4\#1 and shSDC4\#2 (i.e. syndecan-4 knockdown) cell lines. The cells were fixed $6 \mathrm{~h}$ after the confluent cultures were scratched, and the centrosomes were stained with anti $\gamma$-tubulin (green). Nuclei are shown in blue (Hoechst 33258). Individual wide-field fluorescence images were obtained and used to generate panoramic maps for a further analysis of centrosome localization.

Supplementary Figure 10. $\mathrm{Ca}^{2+}$ indicators did not accumulate in the mitochondria. To explore the accumulation of $\mathrm{Ca}^{2+}$ indicators in the mitochondria, cells were loaded by Fura Red and Fluo-4 $\mathrm{Ca}^{2+}$ indicators and MitoTracker Deep Red to visualize mitochondria. (A) Representative confocal images depict the intracellular localization of Fura Red (red) and Fluo4 (green), and MitoTracker Deep Red (blue) in scrambled, shSDC4\#1, and shSDC4\#2 cell lines. (B) Representative diagrams show the signal intensity of the different dyes along the migration axis in scrambled and syndecan-4 knockdown cells. 

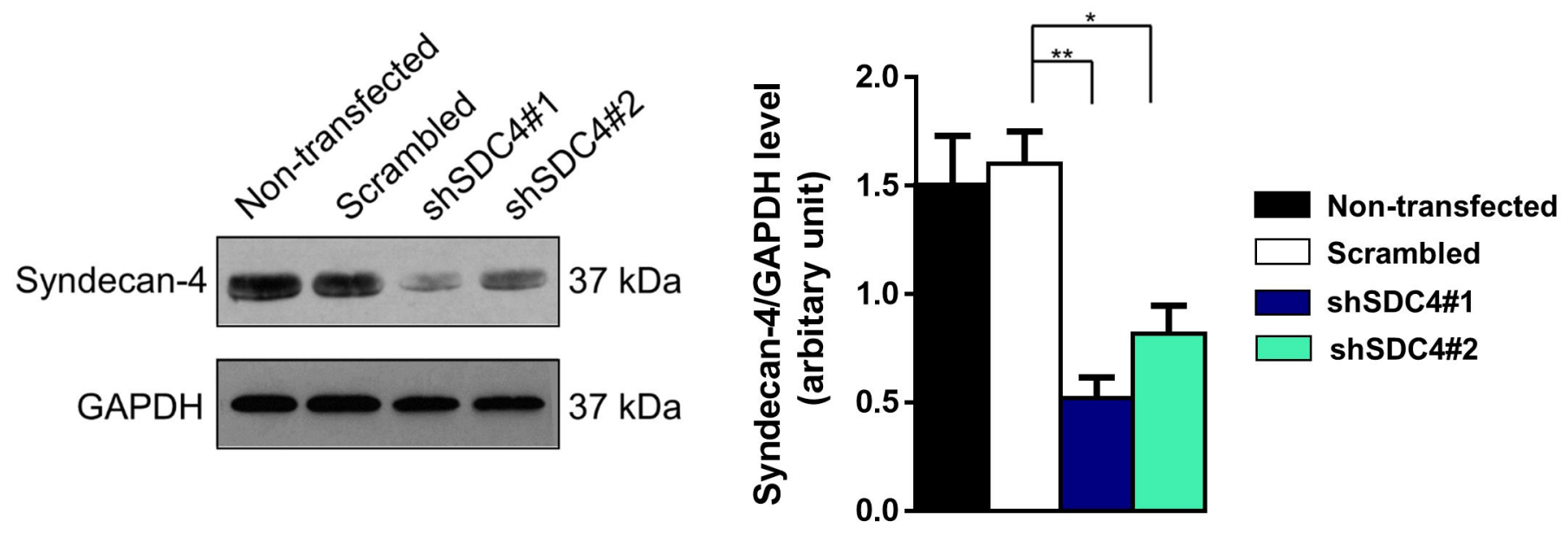

Supplementary Figure 1. 


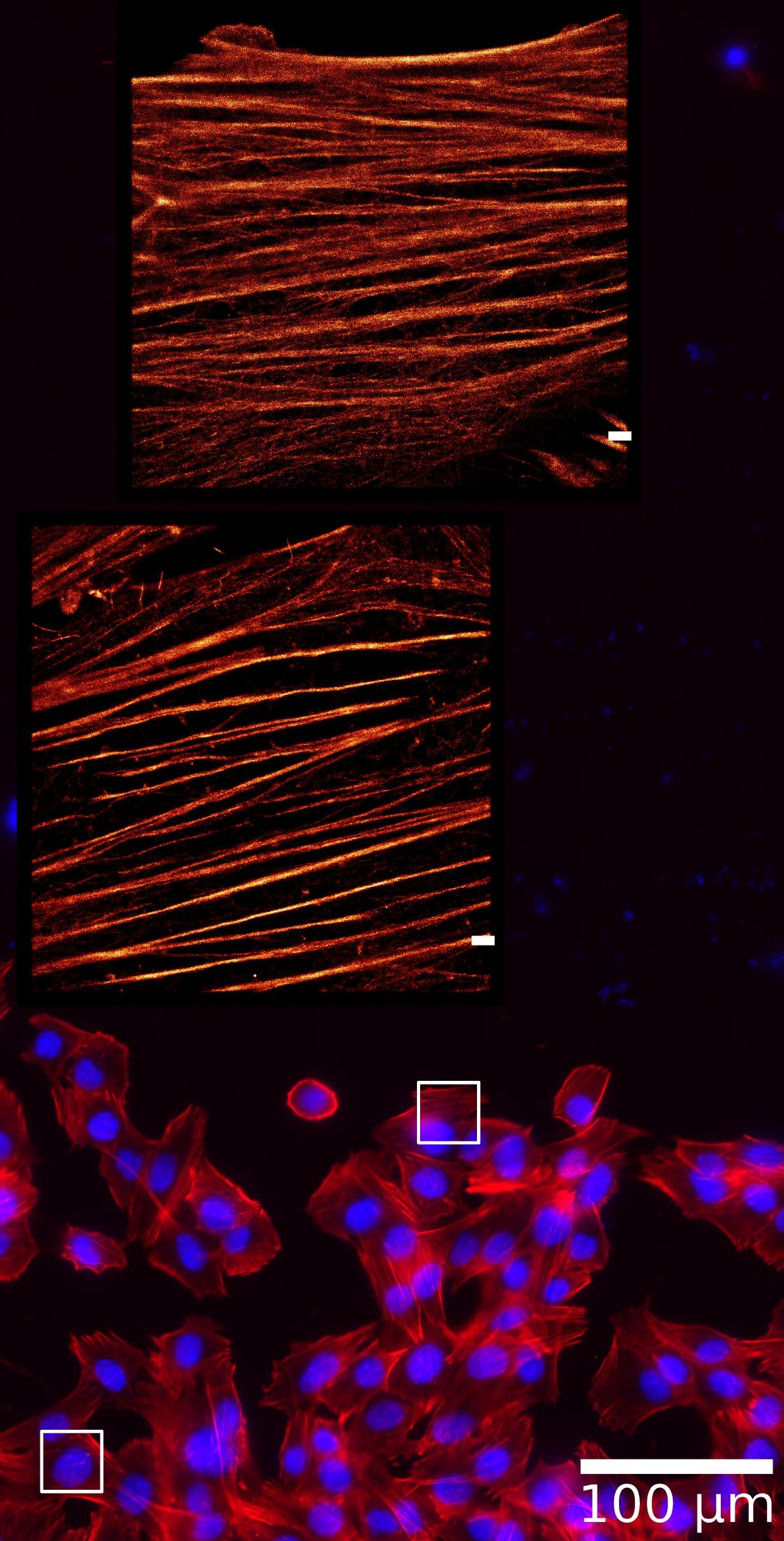




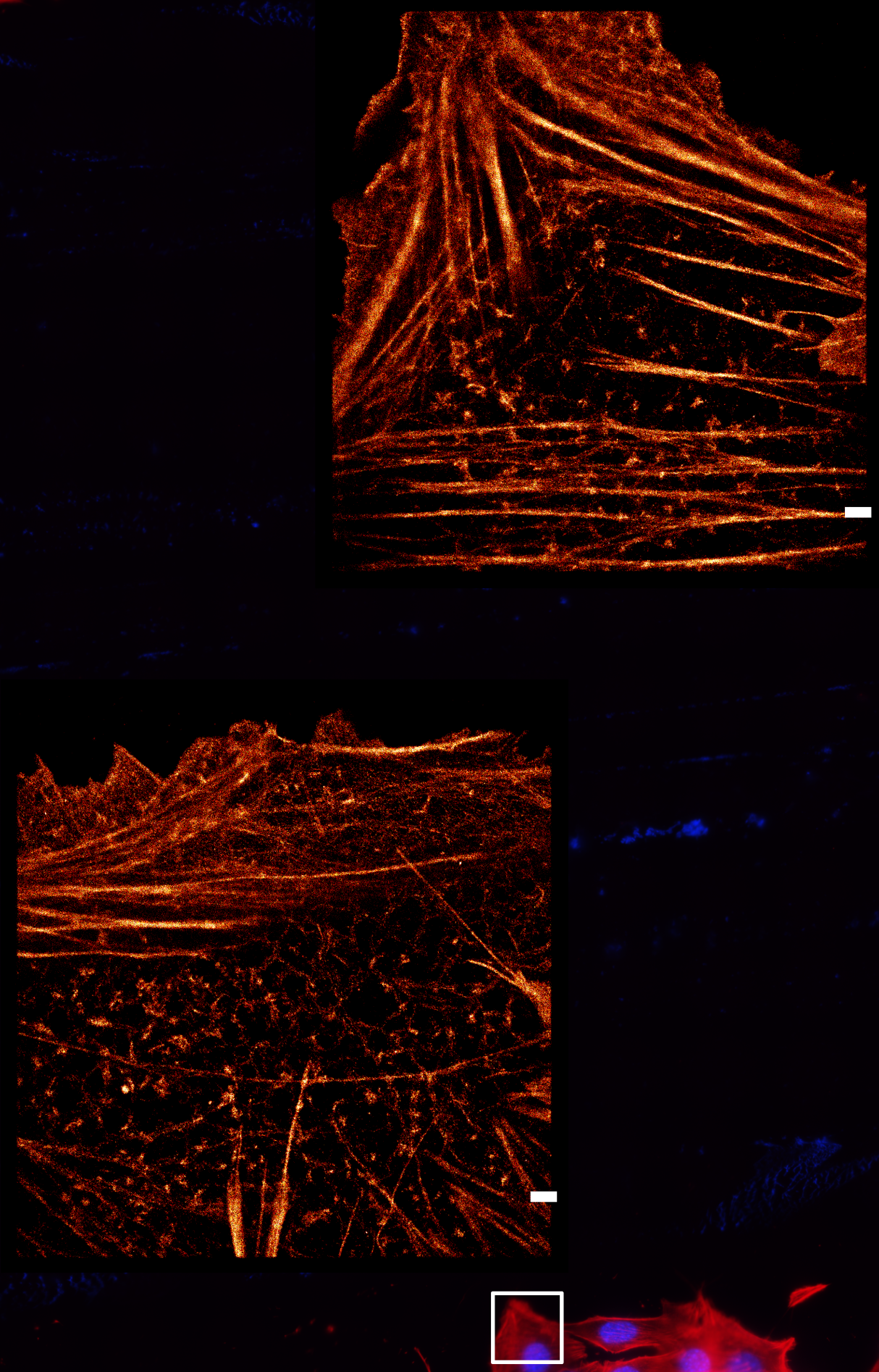

$100 \mu \mathrm{m}$ 
Scrambled

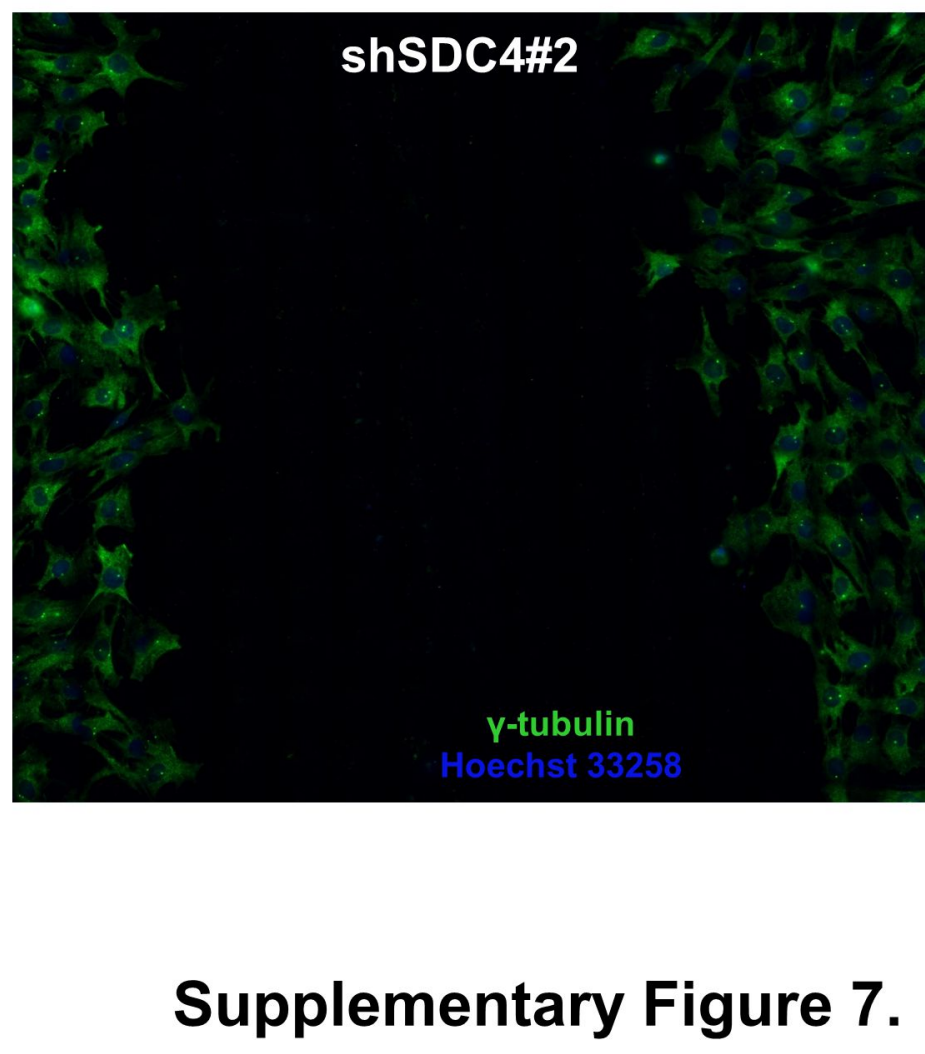


Non-transfected

\section{Scrambled}

Supplementary Figure 8. 
Non-transfected

\section{0um}

\section{Scrambled}

\section{shSDC4\#2}

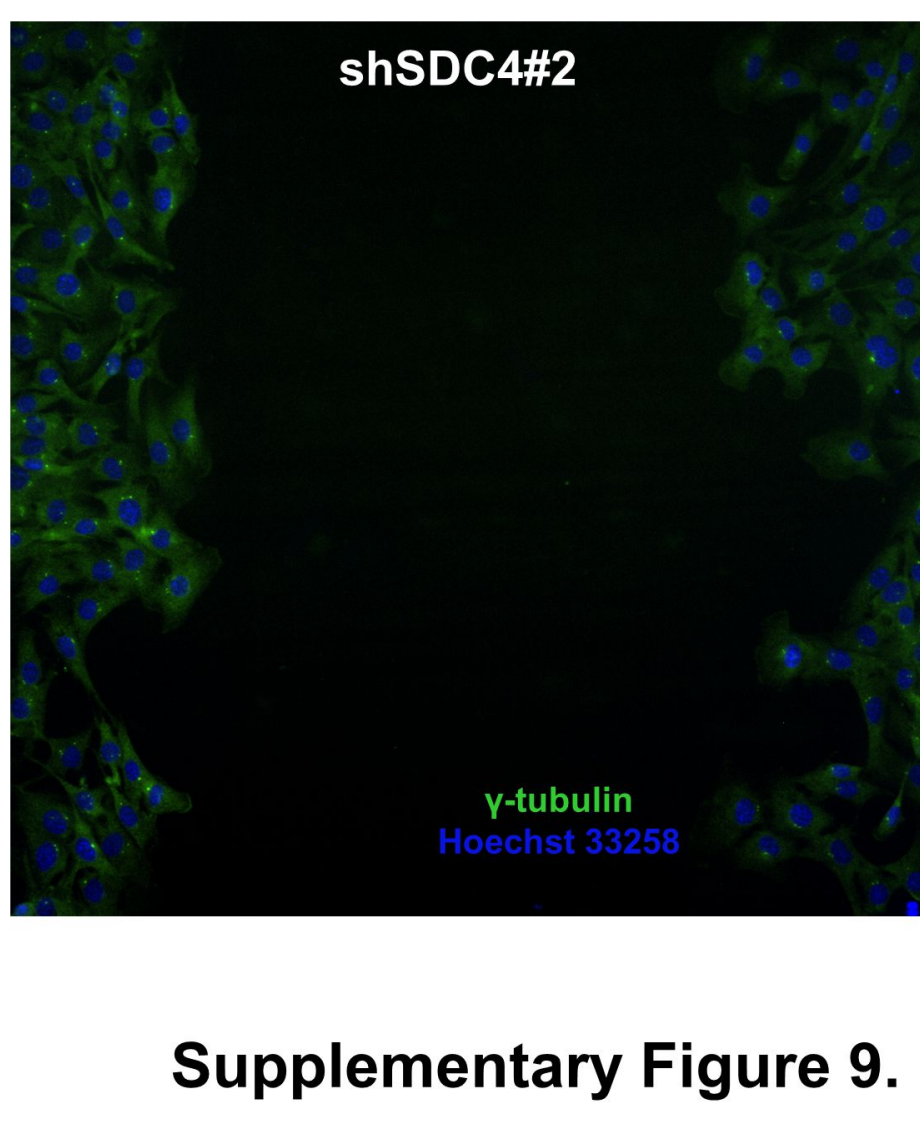



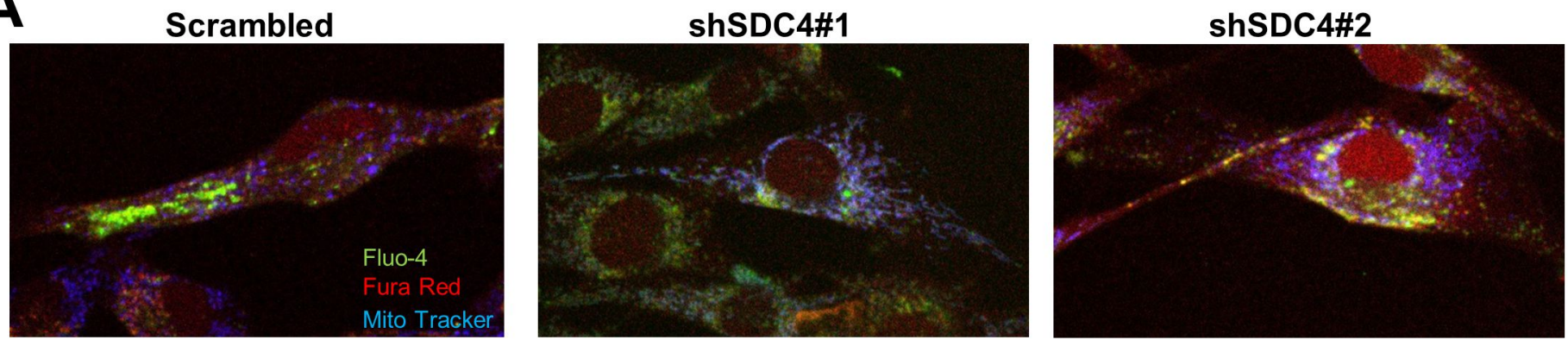

\section{B}

$$
\begin{aligned}
& 0 \\
& \text { S. }
\end{aligned}
$$

Scrambled

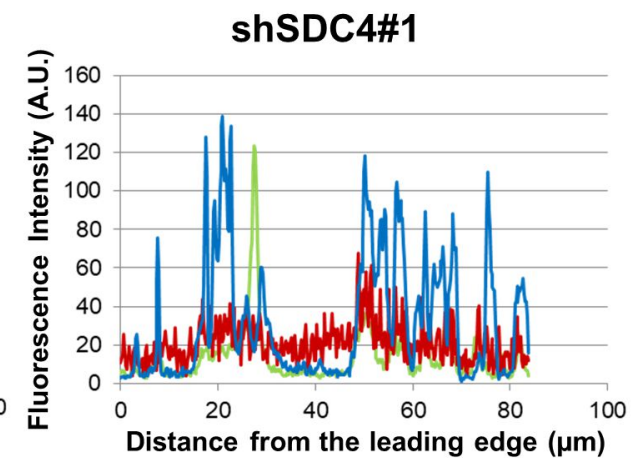

Distance from the leading edge $(\mu \mathrm{m})$

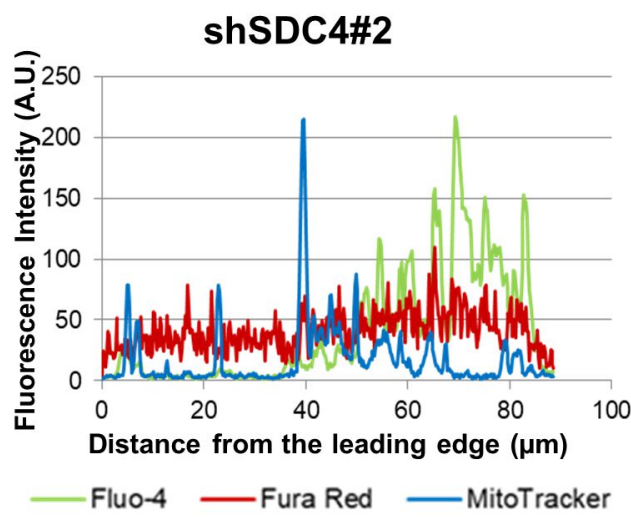

\section{Supplementary Figure 10.}

